

\section{SWITCH-MODE CLASS-E POWER AMPLIFIERS: A CONTRIBUTION TOWARD HIGH PERFORMANCE AND RELIABILITY}

Ali Ghahremani 


\title{
SWITCH-MODE CLASS-E POWER AMPLIFIERS: A CONTRIBUTION TOWARD HIGH PERFORMANCE AND RELIABILITY
}

\author{
DISSERTATION \\ to obtain \\ the degree of doctor at the University of Twente, \\ on the authority of the rector magnificus, \\ Prof.dr. T.T.M. Palstra, \\ on account of the decision of the Doctorate Board \\ to be publicly defended \\ on Wednesday 28 October 2020 at 14.45 uur
}

by

Ali Ghahremani, 21-09-1985, Khodabandeh, Iran 
This dissertation has been approved by:

supervisor

Prof. dr. B. Nauta

co-supervisor

Dr. A.J. Annema

NWO| $\begin{aligned} & \text { Applied and } \\ & \text { Engineering Sciences }\end{aligned}$

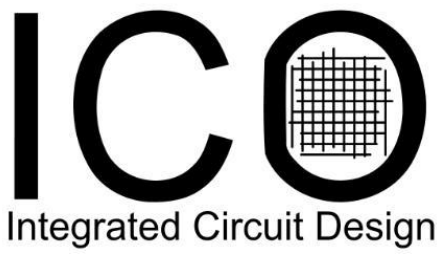

This work is part of the SHERPAs project (no. 12903) and was supported by the Netherlands Organization for Scientific Research (NWO).

Integrated Circuit Design, University of Twente, $7500 \mathrm{AE}$ Enschede, the Netherlands.

Cover design: Shokoufeh Mardanikorani

ISBN: 978-90-365-5039-0

DOI: $10.3990 / 1.9789036550390$

(C) 2020 Ali Ghahremani, The Netherlands. All rights reserved. No parts of this thesis may be reproduced, stored in a retrieval system or transmitted in any form or by any means without permission of the author. Alle rechten voorbehouden. Niets uit deze uitgave mag worden vermenigvuldigd, in enige vorm of op enige wijze, zonder voorafgaande schriftelijke toestemming van de auteur. 


\section{Graduation Committee:}

Chairman / secretary

supervisor:

co-supervisor:

Committee Members prof. dr. J. N. Kok

prof. dr. ir. B. Nauta

dr. A. J. Annema

prof. dr. ir. F.E. van Vliet

prof. dr. ir. P.G.M. Baltus

prof. dr. J.A. Ferreira

prof. dr. L.C.N. de Vreede 
To Shokoufeh and Nickan 


\section{Abstract}

The increasing demands for high data rate necessitate the use of complex modulation schemes that require highly linear transmitters to optimize both the signal quality and the bandwidth usage. These two metrics are usually expressed in terms of error vector magnitude (EVM) and adjacent channel leakage ratio (ACLR). The power amplifiers (PAs), being the last active building block in a transmitter chain, greatly affect the signal quality. Also, typically, PAs are the most power hungry block that have a significant impact on the overall efficiency of a transmitter.

Switch-mode class-E PAs have shown great potential for power efficient amplification of RF signals. Under certain conditions for the transistor voltage and current waveforms, this class of PAs provide (ideally) $100 \%$ efficiency. Also, due to the switched-mode operation of the transistors, class-E PAs are CMOS-friendly and show only a weak dependency on process variations. However, due to incorporating two tuned tanks, the dependency on the load impedance is relatively large, resulting in e.g. load dependent output power, power efficiency, peak voltages and peak (and average) currents which can lead to reliability issues. Load-mismatch can be due to (unintended) changes in the antenna environment or can be due to (intended) load modulation as with e.g. outphasing systems.

This thesis work aims at high performance and reliable class-E PA. The first part of this thesis presents load pull analyses for class-E RF power amplifiers from a mathematical perspective, with analyses and discussions of the effects of the most common non-idealities of class-E PAs. This includes the limited loaded quality factor $\left(Q_{\text {loaded }}\right)$ of the series filter, switch on-resistance, limited quality factor of the DC-feed inductor, load mismatch dependent switch conduction loss and the limited negative voltage excursions (due to e.g. the reverse conduction of the switch transistor for negative voltage excursions). The theoretical findings are backed up by extensive circuit simulations and load pull measurements of a class-E power amplifier implemented in $65 \mathrm{~nm}$ CMOS technology.

Due to switch-mode operation, a single class-E with constant supply only allows phase modulation or On-Off Keying (OOK) modulation. One may use load modulation through outphasing to also enable amplitude modulation. The second part 
of this thesis presents an analysis of outphasing class-E Power Amplifiers (OEPAs), using load-pull analyses of single class-E PAs. This analysis led to an approach that allows to rotate and shift power contours and rotate the efficiency contours to improve the efficiency of OEPAs at deep power back-off, to improve the Output Power Dynamic Range (OPDR) and to reduce switch voltage stress. The theory was validated using a 65nm CMOS demonstration that includes a pcb transmission-line based power combiner.

OEPAs using isolating power combiners and an inverse cosine signal component separator are inherently linear but suffer from low efficiency at power back-off. For high efficiency both at maximum output power and at power back-off, non-isolating power combiners are required. In the third part of this thesis the linearity of OEPAs using non-isolating power combiners is studied theoretically and validated by measurement of an OEPA implemented in a standard 65nm CMOS technology using an off-chip transmission-line based combiner. The developed theoretical model for the linearity is then employed to define digital pre-distortion (DPD) parameters for the implemented OEPA. Using this theory-based DPD and without any AM/AM and $\mathrm{AM} / \mathrm{PM}$ characterizations, the implemented OEPA provides a competitive linearity performance compared to the state of the art OEPAs. -31dB RMS EVM level and below $-30 \mathrm{~dB}$ ACLR were measured for a $13.1 \mathrm{dBm} 6.25 \mathrm{MHz} 30 \mathrm{Mbit} / \mathrm{s} 7 \mathrm{~dB}$ PAPR 64QAM signal with $41.8 \%$ drain efficiency and $33.6 \%$ power added efficiency.

Finally, this thesis introduces a technique to self-protect/self-heal Class-E PAs against the effects of load variations, with only a minor impact on output power and efficiency. To validate the proposed technique, load-pull measurements are conducted on a class-E PA implemented in a standard 65nm CMOS technology, employing an offchip matching network, augmented with a fully automated self-protective/self-healing control loop. It is shown that the proposed self-protective PA can reduce its peak switch voltage from $5.4 \times V_{D D}$ to below $3.8 \times V_{D D}$ for all load mismatch conditions with VSWR up to 19:1 while output power and efficiency are not considerably affected. This allows to reduce the class-E PA's design margins significantly and to choose a higher $V_{D D}$ (to have a higher output power) compared to the case that the selfprotective control loop is disabled. The designed self-protective class-E PA provides $17.5 \mathrm{dBm}$ measured output power from a $1.2 \mathrm{~V}$ supply under nominal load conditions (when all the losses of the matching network are included) and the switch voltage is always below the value allowed by the technology for all load mismatch conditions with VSWR up to 19:1.

Overall, this thesis contributes to design of high performance and reliable switchmode class-E PAs. 


\section{Samenvatting}

De groeiende vraag naar hoge datasnelheden vereist het gebruik van complexe modulatieschema's waarbij zeer lineaire radiozenders nodig zijn om zowel de kwaliteit van het signaal als de bandbreedte te optimaliseren. Deze twee aspecten worden gewoonlijk uitgedrukt in een Error Vector Magnitude (EVM) en een Adjacent Channel Leakage Ratio (ACLR). Door zijn plaats aan het eind van het signaalpad heeft de eindversterker (Power Amplifier (PA)) een grote invloed op de signaalkwaliteit. Gewoonlijk is de eindversterker het systeemelement met de hoogste energieconsumptie, waardoor het een significante invloed heeft op de efficiëntie van het gehele zendsysteem.

Schakelende klasse-E eindversterkers zijn veelbelovend voor het efficiënt versterken van radio-frequente $(\mathrm{RF})$ signalen. Onder specifieke omstandigheden voor de transistorspanning- en -stroomgolfvormen kan deze klasse eindversterker (idealiter) 100\% efficiëntie behalen. Daarnaast zijn klasse-E eindversterkers door hun gebruik van transistoren die uitsluitend schakelen geschikt voor implementatie in CMOS technologiën, met enkel een zwakke afhankelijkheid van productievariaties. Anderzijds is de afhankelijkheid van de belastingsimpedantie relatief groot door het gebruik van resonantiekringen, met als gevolg dat zowel het uitgangsvermogen als de efficiëntie, piekspanningen en piek- en gemiddelde stromen belastingsafhankelijk zijn, wat kan leiden tot betrouwbaarheidsproblemen. Afwijkingen van de nominale belastingsimpedantie kunnen ontstaan door (onbedoelde) veranderingen in de elektromagnetische omgeving van de antenne of door (bedoelde) modulatie van de belastingsimpedantie, zoals bij de outphasing techniek.

Deze thesis is gericht op hoogwaardige en betrouwbare klasse-E eindversterkers for radiofrequente toepassingen. Het eerste deel van deze thesis behandelt load-pull analyses voor klasse-E eindversterkers vanuit een wiskundig oogpunt, inclusief behandeling en discussie van de meestvoorkomde niet-idealiteiten van klasse-E eindversterkers. Tot deze niet-idealiteiten behoren de eindige belaste kwaliteitsfactor ( $\left.Q_{\text {loaded }}\right)$ van de serieresonantiekring, de aan-weerstand van de schakeltransistor, de eindige kwaliteitsfactor van de resonantiekringspoel, belastingsafhankelijke schakelaardissipatie en begrenste negatieve spanningsexcursies (door bijvoorbeeld de geleiding van 
de schakeltransistor voor negatieve spanningsexcursies). De theoretische bevindingen worden ondersteund met uitgebreide circuitsimulaties en load-pull metingen aan een klasse-E eindversterker in een 65nm CMOS technologie.

Door zijn schakelende werking kan een enkele klasse-E eindversterker met een constante voedingsspanning alleen fasemodulatie of On-Off Keying (OOK) realiseren. Het gebruik van de outphasing-techniek maakt het gebruikt van amplitudemodulatie en van complexe modulaties mogelijk. Het tweede deel van deze thesis presenteert een analyse van outphasing klasse-E eindversterkers (Outphasing class-E Power Amplifiers (OEPAs)), aan de hand van een load-pull analyse van individuele klasse-E eindversterkers. Deze analyse leidde tot een techniek waarbij de vermogenscontours op een Smith-kaart geroteerd en geschoven kunnen worden en waarbij de efficiëntiecontours geroteerd kunnen worden. Hiermee kunnen zowel de efficiëntie van OEPAs bij lage vermogens als de Output Power Dynamic Range (OPDR) verbeterd worden, en kan de spanningsstress op de schakelaar verminderd worden. De theorie is bevestigd door middel van metingen op een $65 \mathrm{~nm}$ CMOS implementatie die gebruik maakt van een PCB-transmissielijngebaseerde power combiner.

OEPAs die gebruik maken van isolerende power combiners en een inverse-cosinus signaalcomponentscheider zijn inherent lineair, maar hebben lagere efficiëntie bij lagere uitgangsvermogens. Om hoge efficiëntie te halen bij zowel maximaal als verlaagd vermogen zijn niet-isolerende power combiners nodig. In het derde deel van deze thesis word de lineariteit van dergelijke OEPAs theoretisch onderzocht en geverifiëerd aan de hand van metingen aan een OEPA in een standaard 65nm CMOS technologie, gebruikmakend van een off-chip transmissielijn-gebaseerde power combiner. Het ontwikkelde theoretisch model betreffende de lineariteit word vervolgens gebruikt om digitale pre-distortie (Digital Pre-Distortion (DPD)) parameters te definiëren voor de geïmplementeerde OEPA. Met enkel deze theorie-gebaseerde DPD, zonder gebruik te maken van gemeten AM/AM en AM/PM vervormingskarakterisatie, haalt de geïmplementeerde OEPA een hoge lineariteit, vergelijkbaar met de beste huidige OEPAs. Een RMS EVM niveau van $-31 \mathrm{~dB}$ en een ACLR lager dan -30dB werden gemeten voor een $13.1 \mathrm{dBm} 6.25 \mathrm{MHz} 30 \mathrm{Mbit} / \mathrm{s} 7 \mathrm{~dB}$ PAPR 64QAM signaal met $41.8 \%$ drain efficiëntie en een $33.6 \%$ Power Added Efficiency (PAE).

Als laatste introduceert deze thesis een techniek om klasse-E eindversterkers zichzelf te laten beschermen/repareren tegen belastingsimpedantievariaties, zonder significante impact op het uitgangsvermogen en of de efficiëntie. Om deze techniek te verifiëren zijn metingen gedaan op een klasse-E eindversterker geïmplementeerd in een standaard $65 \mathrm{~nm}$ CMOS proces. Deze eindversterker maakt gebruik van een off-chip matching-netwerk en is uitgebreid met een volledig automatische zelf-beschermende/zelfrepareren regellus. Deze versterker kan zijn piekschakelspanning verlagen van $5.4 \mathrm{x} V_{\mathrm{DD}}$ tot minder dan $3.8 \mathrm{x} V_{\mathrm{DD}}$ voor alle belastingsimpedantieomstandigheden met een VSWR tot 19:1, zonder significante verlaging van uitgangsvermogen noch van de efficiëntie. 
Dit maakt zowel een reductie in de benodigde ontwerpmarges van klasse-E PAs mogelijk, alsmede een verhoging van de voedingsspanning (met bijbehorende uitgangsvermogenverhoging). De ontworpen zelf-beschermende klasse-E eindversterker levert (gemeten) $17.5 \mathrm{dBm}$ uitgangsvermogen uit een $1.2 \mathrm{~V}$ voeding onder nominale condities (waarbij het vermogensverlies van het matching-netwerk is inbegrepen) terwijl de schakelaarsspanning voor alle belastingsimpedantieomstandigheden met een VSWR tot 19:1 lager blijft dan de door de technologie maximaal toegestane waarde.

Met deze innovaties levert het werk dat beschreven is in deze thesis een bijdrage aan het ontwerp van hoogwaardige en betrouwbare schakelende klasse-E eindversterkers voor radiofrequente toepassingen. 


\section{Contents}

Abstract

Samenvatting $\quad$ iii

1 Introduction 1

1.1 Power Amplifiers: General Definitions . . . . . . . . . . . . . . 2

$1.2 \mathrm{RF}$ Antennas . . . . . . . . . . . . . . . . . . . . 3

1.3 BPSK modulation . . . . . . . . . . . . . . . . . . 6

1.4 Modulation bandwidth issues and Pulse shaping . . . . . . . . . 7

1.5 Quadrature Amplitude Modulation (QAM) . . . . . . . . . . 8

1.6 Spectral Regrowth . . . . . . . . . . . . . . . . . 9 9

1.7 Error Vector Magnitude . . . . . . . . . . . . . . . . . . . 11

1.8 Peak to Average Power Ratio (PAPR) of modulated signals . . . . . . 11

1.9 Thesis organization . . . . . . . . . . . . . . . . . 13

2 Power Amplifiers: Methods of Amplification $\quad 17$

2.0.1 Linear PAs . . . . . . . . . . . . . . . . 17

2.0 .2 Class J PAs . . . . . . . . . . . . . . . . . . . 21

2.0 .3 Class F PAs . . . . . . . . . . . . . . . 22

2.0.4 Class D PAs . . . . . . . . . . . . . . . 22

2.0 .5 Class E PAs . . . . . . . . . . . . . . . . . . 24

2.0.6 Doherty structure . . . . . . . . . . . . . . . 24

2.0.7 Envelope Elimination and Restoration (EER) . . . . . . . . 28

2.0.8 Envelope Tracking $(\mathrm{ET})$. . . . . . . . . . . . . . . . 29

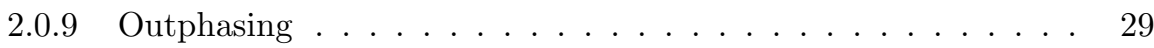

2.1 PA class selection . . . . . . . . . . . . . . . . . 32

3 Load-pulling and PA Break-down $\quad 37$

3.1 Linear PAs and load-pulling . . . . . . . . . . . . . . . . . 37

3.2 Transistor breakdown mechanisms . . . . . . . . . . . . . . . 40 
3.2 .1 Gate oxide breakdown . . . . . . . . . . . . . . . 40

3.2 .2 Hot carrier degradation $\ldots \ldots \ldots . \ldots . \ldots . \ldots$

3.2 .3 Punch through $\ldots \ldots \ldots \ldots \ldots$

3.2 .4 Drain-bulk breakdown . . . . . . . . . . . . . . 41

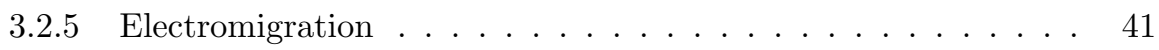

4 Load-mismatch Sensitivity of Class-E Power Amplifiers 45

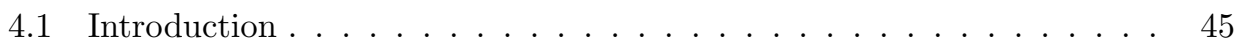

4.2 Class-E Power Amplifier Basics . . . . . . . . . . . . . . . 48

4.3 Non-ZVS and non-ZSS Class-E PA . . . . . . . . . . . . 50

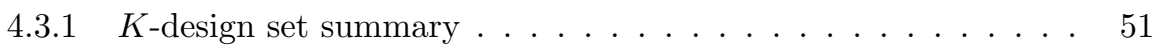

4.3 .2 Waveform maxima . . . . . . . . . . . . . 52

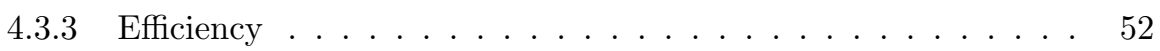

4.3.4 Non-ZVS and non-ZSS class-E summary . . . . . . . . . . 53

4.4 Antenna Load-Mismatch Effects On Class-E Performance . . . . . . . 53

4.4 .1 Effects on PA load . . . . . . . . . . . . . . . 53

$4.4 .2 \quad$ K-design set. . . . . . . . . . . . . . . . . 54

4.4.3 Case study: high power, high efficiency Class-E PA under antenna load-mismatch conditions . . . . . . . . . . . . . 55

4.5 Reliability issues and safe operating region . . . . . . . . . 63

4.5.1 Effects of the parameters $d$ and $q \ldots \ldots \ldots \ldots \ldots \ldots$

4.6 Impact of non-ideal effects . . . . . . . . . . . . . . . 65

4.6.1 Shunting negative voltage excursions across the switch . . . . 66

4.6.2 Load dependent switch conduction loss $(m) \ldots \ldots \ldots 68$

4.6 .3 Load-pull contours . . . . . . . . . . . . . . . . . . . 68

4.7 Measurements . . . . . . . . . . . . . . . . . . . . . . . . . . 69

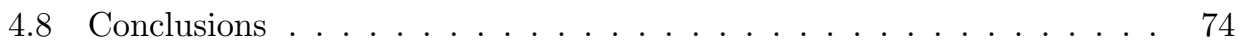

5 Outphasing Class-E PAs: From Theory to Back-off Efficiency Im$\begin{array}{ll}\text { provement } & \mathbf{7 9}\end{array}$

5.1 Introduction . . . . . . . . . . . . . . . . . . . 79

5.2 Class-E Power Amplifier Basics . . . . . . . . . . . . . . . . . . . . 82

5.2 .1 Load-pulling class-E PAs . . . . . . . . . . . . . . . 83

5.2.2 Effect of changing $\mathrm{q}$ and $\mathrm{d}$ on the load-pull contours . . . . . 84

5.3 Simplified Theory of OEPAs . . . . . . . . . . . . . 85

5.3.1 Output Vectors' Amplitude Mismatch . . . . . . . . . . . . 87

5.4 Back-off efficiency improvement technique . . . . . . . . . . . . . 89

5.4 .1 Rotation . . . . . . . . . . . . . . . . . . . . 89

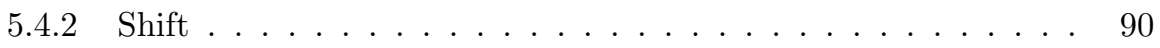

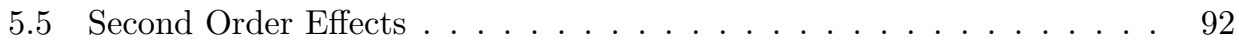


5.5.1 Switch conduction loss . . . . . . . . . . . . . . 92

5.5.2 Limited quality factor of the DC-feed inductor L . . . . . . . . 94

5.6 Implementation in 65nm CMOS technology . . . . . . . . . . . . . . . 94

5.7 Measurement Results . . . . . . . . . . . . . . . . . . . . . 97

5.8 Conclusion . . . . . . . . . . . . . . . . . 103

6 Linearity of Outphasing Class-E PAs 109

6.1 Introduction . . . . . . . . . . . . . . . . . . . . . . . 109

6.2 Theoretical model for the linearity of OEPAs . . . . . . . . . . . 111

6.3 Experimental verification . . . . . . . . . . . . . 116

6.4 Conclusion . . . . . . . . . . . . . . . . . . 120

7 Augmentation of Class-E PA reliability under load mismatch conditions

7.1 Introduction . . . . . . . . . . . . . . . . . . . 123

7.2 Class-E PAs under (non-)nominal conditions . . . . . . . . . . . 125

7.2.1 Effects of the $q$ parameter on PA behavior . . . . . . . . . . . 127

7.3 Implementation . . . . . . . . . . . . . . . . . . . . 127

7.3.1 Measurement results with fixed q parameter . . . . . . . . . . . 128

7.3.2 Self-healing Class-E PA . . . . . . . . . . . . . . . 130

7.4 Conclusions . . . . . . . . . . . . . . . . . . . . 131

8 Summary and recommendations for future research $\quad 135$

8.1 Summary and original contributions . . . . . . . . . . . . . . 135

8.2 Recommendations . . . . . . . . . . . . . . . . 137

A Effect of PA impedance at the harmonics 139

B Effect of changing duty cycle on the load-pull contours 143

C Extension of the load-pull contours toward negative impedances 145

$\begin{array}{lr}\text { Acknowledgments } & 147\end{array}$

$\begin{array}{ll}\text { List of publications } & 149\end{array}$

Bibliography $\quad 149$ 


\section{Chapter 1}

\section{Introduction}

Radio frequency power amplifiers (RF PAs) are the most power hungry building blocks in transmitter chains. PAs are used to convert dc (supply) power into a desired level of modulated RF output power specified by the target application/standard. PAs, while (seemingly) simple at the schematic level, are not just linear amplifiers that are driven into saturation. In fact there are different types of amplification techniques employed by different PA configurations [1].

With the introduction of silicon bipolar transistors at the end of the $60 \mathrm{~s}$, solid-state RF PAs started to replace the old-fashion vacuum tube PAs. Nowadays several solidstate technologies are available for both discrete (such as GaAs) and integrated (such as thick-oxide or LDMOS transistors offered by CMOS technology) implementations of PAs. In this thesis the focus is on integrated CMOS PAs.

Dealing with complex modulated signals in modern communication systems, such as Orthogonal Frequency Division Multiplexing (OFDM) or 64 Quadrature Amplitude Modulation (QAM) [2-4], imposes difficult design challenges on designing efficient PAs. In particular, there is a widely accepted trade-off between PA linearity and efficiency. Intrinsically so-called linear PAs, such as class-A PAs, can perform linearly but the efficiency of such PAs is at best $50 \%$. On the other hand switch-mode class-E PAs are non-linear PAs that can provide up to (ideally) 100\% efficiency [1]. Over the past decades significant effort has been put into attempts at breaking the trade-off between linearity and efficiency of PAs [1] by e.g. using a switch-mode PAs along with linearization techniques. For instance by using class-E PAs in an outphasing structure, linear operation can be provided with (ideally) $100 \%$ efficiency at maximum output power as well as at deep power back-off [5].

So far many RF transmitter blocks have been implemented successfully in CMOS. For PAs, despite the currently existing types of implementations, some remaining issues still need to be addressed. For example, the design of a PA can be challenging 
when it comes to practical applications. For example the antenna impedance, which normally is considered to be constant, can vary strongly [6,7]. For linear PAs, such as class-A PAs with input power control to ensure that the PA keeps operating as a linear $\mathrm{PA}$, it is shown that this can degrade the output power and the PA efficiency while the reliability is not compromised. However, at the start of the research described in this thesis, the effect of load-mismatch on performance and reliability of switch-mode class-E PAs was largely unknown.

This chapter serves as an introduction into this thesis and provides some general concepts related to RF PAs that will be used in the next chapters. Discussions on antenna mismatch, complex modulation schemes such as 64 QAM and the challenges that are imposed in PA design are briefly reviewed. For more information we refer to [2-4].The outline of this thesis is also given in this chapter.

\subsection{Power Amplifiers: General Definitions}

In this section some general definitions relating to PAs and used throughout this thesis are reviewed. For more comprehensive details we refer the readers to $[1,4]$.

A simplified schematic of a PA is shown in Fig. 1.1(a). A matching network is used to provide the optimum impedance ${ }^{1}$ for the PA to deliver the desired output power $\left(P_{\text {out }}\right)$ into the load for a supply voltage $V_{D D}$. There are two different definitions for the efficiency, Drain Efficiency (DE) and Power Added Efficiency (PAE), defined as

$$
\begin{aligned}
& \mathrm{DE}=\frac{P_{\text {out }}}{P_{s}} \\
& \mathrm{PAE}=\frac{P_{\text {out }}}{P_{s}+P_{\text {in }}}
\end{aligned}
$$

where $P_{s}$ is the supply power and $P_{i n}$ is the input power required to drive the PA.

For high order amplitude modulated signals, such as 64 QAM, linear PAs are required. In this work we consider narrow-band applications; for which the memory (dynamic) effects can be neglected and the desired relation between the input and output phasors $V_{\text {in }}$ and $V_{\text {out }}$, respectively, can be written as (Fig. 1.1(a))

$$
V_{\text {out }}=K \cdot V_{\text {in }}
$$

where $K$ is a complex constant factor. However, due to incorporating active, intrinsically non-linear devices in PAs (i.e. transistors), the complex factor $K$ is not constant and both its amplitude and phase is signal dependent yielding distortion. These dependencies are (conventionally) characterized by the AM/AM and AM/PM distortions, which represents respectively the amplitude and phase of the (ideally) constant

\footnotetext{
${ }^{1}$ More details on the optimum impedance for the PAs are given in chapter 2.
} 


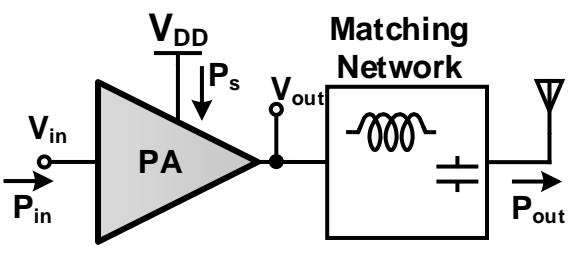

(a)
Linear

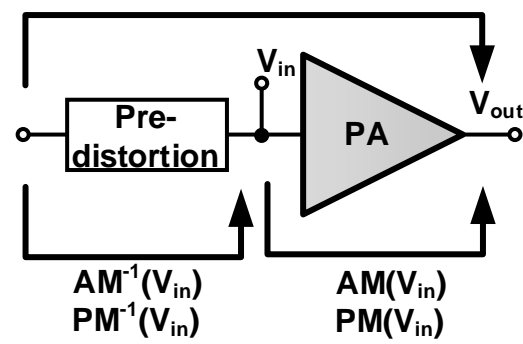

(b)

Figure 1.1: (a) Top level schematic of a PA. (b) Concept of pre-distortion.

$K$ as a function of input amplitude $\left|V_{i n}\right|$ or input power $P_{i n}$. These characterized $\mathrm{AM} / \mathrm{AM}$ and $\mathrm{AM} / \mathrm{PM}$ information, then subsequently, can be used to pre-distort the PA, shown in Fig. 1.1(b), to provide a linear operation and to have a linear amplified replica of the input signal at the PA's output. Yet, to show the performance of the pre-distortion, other metrics such as Adjacent Channel Leakage Ratio (ACLR) and Error Vector Magnitude (EVM) are used (will be discussed later in this chapter).

\subsection{RF Antennas}

Since the first practical demonstration of wireless technology by Guglielmo Marconi in 1901, antennas have become key building blocks of transmitters. An antenna is a single device that allows for transmission of RF energy through free space. Nevertheless, in many instances, not so much attention is paid to them in the system design.

Antennas, in the initial RF system design phase, are modeled by a constant (typically) $50 \Omega$ impedance [4]. At the transmitter side of a wireless system, a PA is required to drive the antenna. In order to achieve maximum energy transfer between the PA and the antenna, the input impedance of the antenna must match the output impedance of the feeder ${ }^{2}$ that drives the antenna. Otherwise, a reflected wave will be generated at the antenna side that travels back to the PA and can degrade the overall performance of the transmitter.

The radiation pattern of the antenna describes the relative strength of the radiated field in various directions from the antenna at a constant distance. Any object placed

\footnotetext{
${ }^{2}$ The feeder can be the PA itself or a transmission line/matching network that are typically used between the PA and the antenna.
} 
in the near field of the antenna will alter its radiation pattern and as a result will change the antenna impedance $[6,7]$.

A common measure of the quality of matching between an antenna and the transmission line (that feeds the antenna) is the Voltage Standing Wave Ratio (VSWR) [8]. For this, we first need to introduce the reflection coefficient at the feed point of the antenna $\left(\Gamma_{Z A}\right)$ which is defined as the fraction of the (incident) power $P_{i}$ that is reflected $\left(P_{r}\right)$ from the antenna. For the schematic of Fig. 1.2(a), it can be shown that [8]

$$
\Gamma_{Z_{A}}=\frac{P_{r}}{P_{i}}=\frac{Z_{A}-50 \Omega}{Z_{A}+50 \Omega}
$$

where $Z_{A}$ is the antenna impedance when the environment interacts in the near field of the antenna and the $50 \Omega$ is the ideal (typical) antenna impedance. For any $Z_{A}$ with $\operatorname{Re}\left\{\mathrm{Z}_{\mathrm{A}}\right\}>=0$, we have $\left|\Gamma_{Z A}\right| \leq 1$.

The non-zero reflected power from the antenna creates standing waves along the transmission line [8] with amplitude depending on the distance $l$ from the antenna. VSWR is defined as the ratio of the peak amplitude of the standing wave to its minimum amplitude, shown in $1.2(\mathrm{~b})$. It can be shown that [8]

$$
\mathrm{VSWR}_{Z_{\mathrm{A}}}=\frac{1+\Gamma_{Z_{A}}}{1-\Gamma_{Z_{A}}}
$$

For a matched antenna $Z_{A}=50 \Omega, \Gamma_{Z_{A}}=0$ and $\mathrm{VSWR}_{\mathrm{Z}_{\mathrm{A}}}=1$.

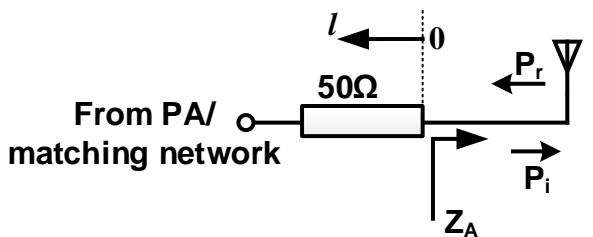

(a)

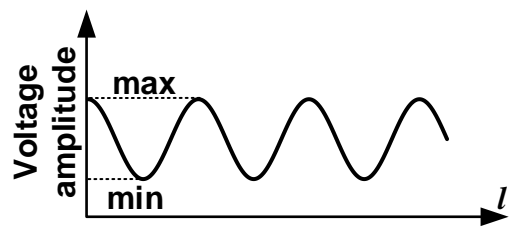

(b)

Figure 1.2: (a) Antenna and its 50 $\Omega$ feeder. (b) Illustration of VSWR. Amplitude of the standing wave depends on the distance $l$ from the antenna.

A common method to represent impedances is to use the Smith chart representation. The Smith chart can be used to simultaneously display multiple parameters including impedances, admittances, constant power and efficiency contours and regions for safe operation of a PA. For this, all these parameters should be translated into reflection coefficients. In this thesis we use Smith chart frequently which is shown in Fig. 1.3 in detail. In the remainder of this thesis we drop the details for simplicity. 
1.2. RF Antennas

\section{Smith Chart}

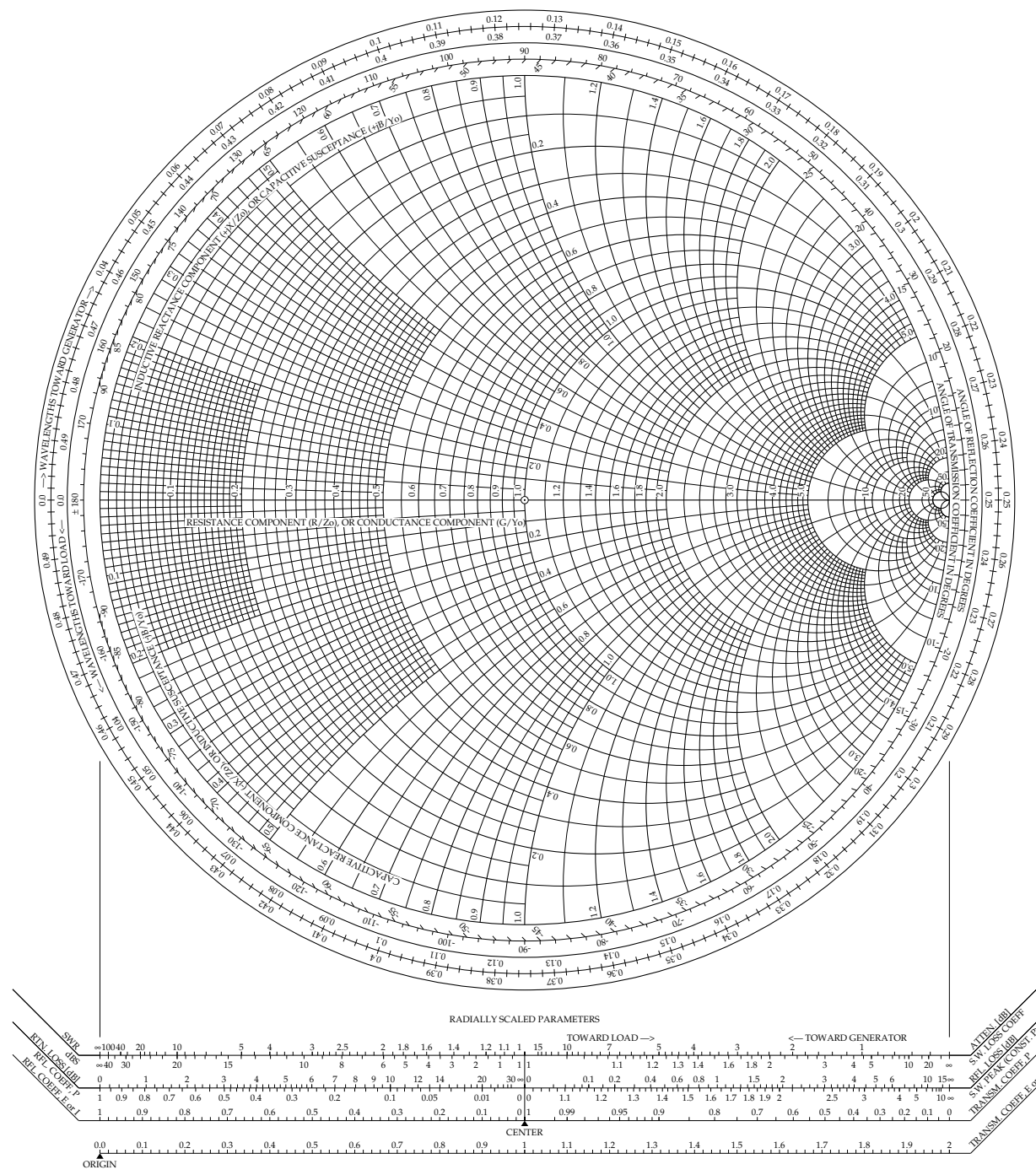

Figure 1.3: An impedance Smith chart (with no data plotted). [9]. Constant real and imaginary contours are shown. 
Basically, Smith chart is a unit-radius circle that each point at or within this circle corresponds to a reflection coefficient $\Gamma$ (note that $|\Gamma| \leq 1^{3}$ ). Using (1.4), the center of the Smith chart $(\Gamma=0)$ corresponds to $Z_{A}=50 \Omega$, matched antenna. At the right (left) hand side, $\Gamma=1(\Gamma=-1)$ hence $Z_{A} \rightarrow \infty\left(Z_{A}=0\right)$, an open (short) circuit at the antenna feed point and at the upper/lower side of the horizontal axis the imaginary part of the antenna impedance is positive/negative (inductive/capacitive). For more details, we refer the interested reader to e.g. [8].

\subsection{BPSK modulation}

Let us assume we wish to transmit a baseband data, toggling between -1 and 1 , as shown in Fig. 1.4(a). We can simply use a carrier at frequency $\omega_{0}$ and e.g. modulate its phase (shown in Fig. 1.4(a)). The resulting signal is denoted as Phase Shift keying (PSK) or Binary PSK (BPSK).

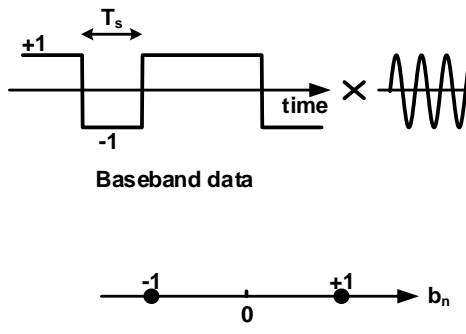

(b)

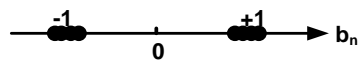

(c)

Figure 1.4: (a) Generation of BPSK signals. Signal constellation for (b) ideal and (c) noisy BPSK signal [4].

To visualize modulation schemes and the effect of nonidealities on them, signal constellations are used. For example, for the BPSK modulation of Fig. 1.4(a), we have

$$
x_{B P S K}=b_{n} \cos \left(\omega_{0} t\right) ; b_{n} \in\{-1,1\} .
$$

The BPSK signal has cosine function as its basis function and can be fully characterized by possible values of the symbols $b_{n}$, shown in Fig. 1.4(b) and are denoted as the

\footnotetext{
${ }^{3}$ The Smith chart is most frequently used at or within the circle. However, the remainder is still relevant in e.g. oscillator design and stability analysis
} 
signal constellation. In practice, to plot the constellation diagram of signals used in a communication link, the signals are demodulated at the receiver and the baseband data waveforms similar to e.g. Fig. 1.4(a) are reproduced and sampled at $T_{s} / 2$ and $T_{s}$ is (information) pulse width. Any nonidealities such as distortion of the transmitters or noise at the receiving side of the communication link make the constellation points to look fuzzy (Fig. 1.4(c)).

\subsection{Modulation bandwidth issues and Pulse shap- ing}

The BPSK modulation scheme with the square wave baseband pulse shaping, shown in Fig. 1.4(a), suffers from two issues. Firstly, due to the sharp transition of the time domain baseband signal the energy (of the signal) is spread over an infinite bandwidth. However, the communication channels that the signal is being transmitted through are band limited. As a result, for the BPSK input signals, the output of these channels exhibit an exponential tail in time domain that becomes longer as the channel bandwidth (BW) decreases. Then at the sampling moments, at the receiver to determine each symbol $(-1$ or +1$)$, the resulting value is corrupted by decaying tails created by previous symbols. This effect is called Intersymbol Interference (ISI) [4].

Secondly, performing Fourier analysis results in a sinc-shaped Power Spectral Density (PSD) of the signal, shown in Fig. 1.5(a); the signal has a considerable amount of energy in the side lobes of the sinc function. In communication standards the energy of the signal must be confined to channel BW. For a channel with $\mathrm{BW}=$ $2 / T_{s}$, even if the resulting ISI (due to small BW of the channel compared to the BW of the signal) can be accepted, the side lobes of the sinc function fall outside the channel and normally are restricted by the standards.

To resolve these issues, the BW of the baseband signal should be reduced before up conversion and transmission through the communication channel. The tightest spectrum can be obtained by using time domain sinc pulses, shown in Fig. 1.5(b). The resulting PSD of the sinc pulse shaping is (only) non-zero within $B W=1 / T_{s}$. Furthermore, at the receiver side, by sampling at exactly integer multiples of $T_{s}$, the ISI can be eliminated (all other pulses have a zero-crossing at these sample points). Yet, a small timing error at the sampling moments can cause a large ISI due to small roll-off of the sinc function by time.

A common pulse shape addressing this issue, denoted as raised-cosine and shown in Fig. 1.5(c), is expressed in time domain as follows

$$
p(t)=\frac{\sin \left(\pi t / T_{s}\right)}{\pi t / T_{s}} \frac{\cos \left(\pi \alpha_{r} t / T_{s}\right)}{1-4 \alpha_{r}^{2} t^{2} / T_{s}^{2}}
$$


where the parameter $\alpha_{r}$ is the so-called roll-off factor and determines how close $p(t)$ is to a sinc function in time domain. For $\alpha_{r}=0$, the pulse reduces to a sinc pulse. The parameter $\alpha_{r}$ is also, sometimes, called as excess BW as it shows how much extra BW the shaped pulse requires compared to a sinc pulse.
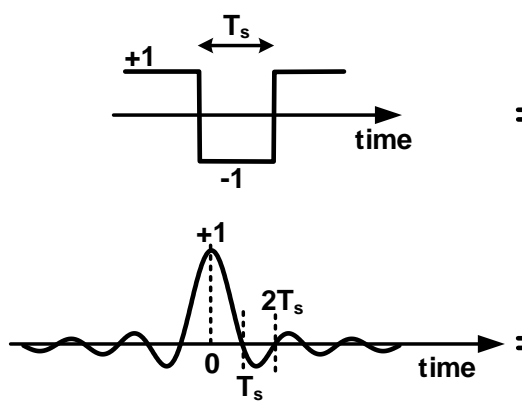

(a)
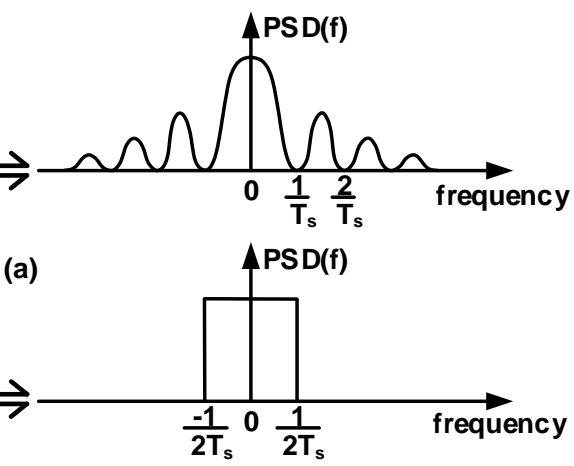

(b)

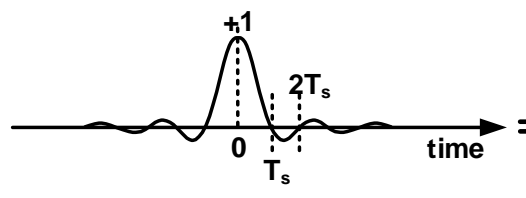

$A P S D(f)$

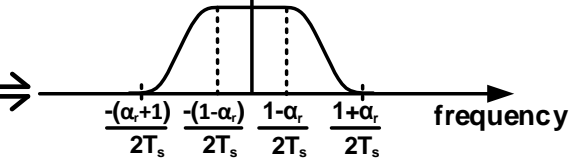

(c)

Figure 1.5: (a) BPSK signals and its spectrum. (b) Sinc pulse and its spectrum. (c) Raised-cosine pulse and corresponding spectrum [4].

\subsection{Quadrature Amplitude Modulation (QAM)}

Quadrature modulation is a technique to improve the throughput of communication links (for a given channel bandwidth) by using two orthogonal carriers, for our applications, $\cos \left(\omega_{0} t\right)$ and $\sin \left(\omega_{0} t\right)$. For the case of binary baseband data, the concept is illustrated in Fig. 1.6(a). A serial-to-parallel (S/P) converter separates the even- and odd-numbered bits and each group of bits is carried by one of the orthogonal carriers. Quadrature modulation reduces the occupied BW by a factor of two or, it enhances the throughput by a factor of two compared to single-carrier modulation case for the same BW. This is simply because the $\mathrm{S} / \mathrm{P}$ converter stretches each bit duration by a 
factor of two before modulating the carriers.

To obtain the constellation diagram, the output signal $x(t)$ in Fig. 1.6(a) can be written as $x(t)=b_{2 n} \cos \left(\omega_{0} t\right)+b_{2 n+1} \sin \left(\omega_{0} t\right)$, where $n$ is the input bits' index and $\left\{b_{2 n}, b_{2 n+1}\right\} \in\{-1,1\}$. Using two orthogonal axes for the two orthogonal carries, the constellation diagram of Fig. 1.6(b) can be drawn. The horizontal axis (corresponding to the cosine carrier) is denoted by I (for in-phase) and the vertical axis (corresponding to the sine carrier) is denoted by $\mathrm{Q}$ (for quadrature). Each constellation symbol, corresponding to two consecutive bits, carries two bits. This modulation scheme, then, is denoted as 4 QAM (QPSK is also used in literature because this is the quadrature version of (binary) PSK).

Allowing the input data take more amplitude levels (not only $\{-1,+1\}$ ), higher QAM signals can be created. For $\left\{b_{2 n}, b_{2 n+1}\right\} \in\{ \pm 0.5, \pm 1.5, \pm 2.5, \pm 3.5\}$, we obtain a 64 QAM signal, of which the corresponding constellation diagram is shown in Fig. 1.6(c). For this constellation diagram, six consecutive bits in the binary baseband stream are grouped and, accordingly, each quadrature component of the carrier is allowed to have eight possible amplitudes. Then, each constellation symbol carries six bits.

\subsection{Spectral Regrowth}

For a communication channel with bandwidth $\mathrm{BW}_{\mathrm{ch}}$, to transmit at symbol rate $1 / T_{s}$, where $\mathrm{BW}_{\mathrm{ch}}=1 / T_{s}$, sinc pulse shaping is necessary to limit out of channel transmission. Yet, for a better ISI performance, raised-cosine pulse shaping is more popular and a roll-off factor $\alpha_{r}$ results in an excess BW by a factor of $\alpha_{r}$. For example, the PSD of a 64 QAM amplitude modulated signal with raised-cosine pulse shaping with $\alpha_{r}=0.25$ and $10 \mathrm{MSymb} . / \mathrm{sec}$ data rate $(60 \mathrm{Mbit} / \mathrm{sec})$ and $12.5 \mathrm{MHz}$ bandwidth (25\% excess BW) is shown in Fig. 1.7(a). It can be seen that, almost, the whole energy of the signal is inside the (for this example) $12.5 \mathrm{MHz}$ communication channel.

For an amplitude modulated signal such as 64 QAM, any distortion in the transmit path can result in spectral regrowth, i.e. out-of-channel emissions. To illustrate the underlying mechanism, consider an amplitude modulated signal $x(t)$ applied to a nonlinear system that exhibits a third-order memoryless nonlinearity. Writing $x(t)$ as

$$
x(t)=x_{I}(t) \cos \left(\omega_{0} t\right)+x_{Q}(t) \sin \left(\omega_{0} t\right)
$$

where $x_{I}$ and $x_{Q}$ are the baseband I and $\mathrm{Q}$ components. At the output of the nonlinear system

$$
\begin{aligned}
y(t) & =a_{3} x^{3}(t)+\ldots \\
& =a_{3} x_{I}^{3}(t) \frac{\cos \left(3 \omega_{0} t\right)+3 \cos \left(\omega_{0} t\right)}{4}-a_{3} x_{Q}^{3}(t) \frac{-\cos \left(3 \omega_{0} t\right)+3 \sin \left(\omega_{0} t\right)}{4}
\end{aligned}
$$



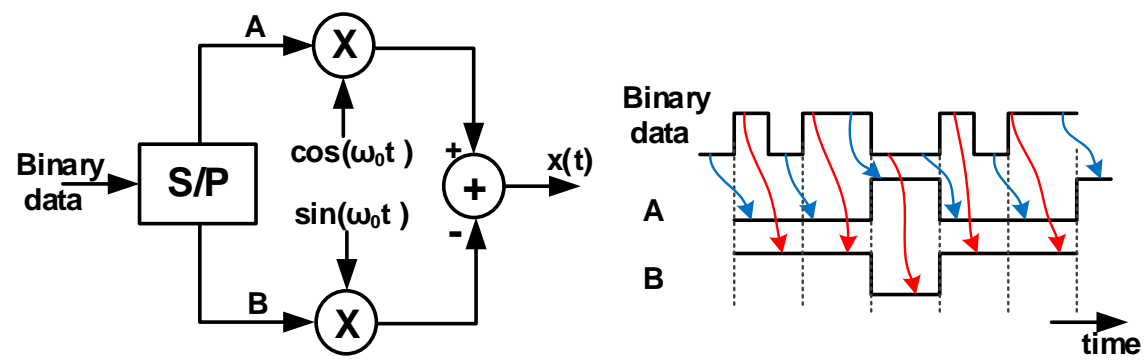

(a)

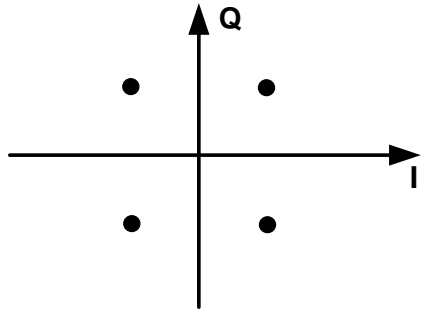

(b)

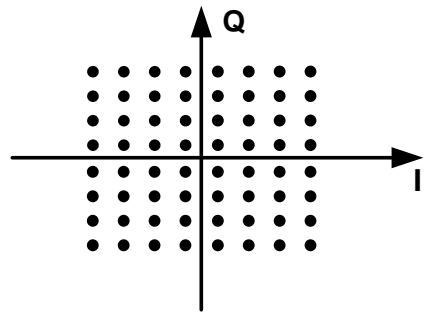

(c)

Figure 1.6: (a) illustration of QAM concept. Constellation diagram for (b) 4 QAM (QPSK) and (c) 64 QAM signal [4].

Then the output signal contains the spectra of $x_{I}^{3}(t)$ and $x_{Q}^{3}(t)$, centered around $\omega_{0}$ on top of the spectra of the desired signal components $x_{I}(t)$ and $x_{Q}(t)$. Since these components exhibit a broader spectrum than those of $x_{I}(t)$ and $x_{Q}(t)$, the resulting output spectrum of an amplitude modulated signal that passes through a nonlinear system grows.

In practice, it is not possible to design a perfectly linear system (there is always a residual amount of nonlinearities in a system that has a power gain $>1$ ). In communication standards, maximum limits are specified for the power that can be transmitted out of the allocated channel. These limits are given as a function of frequency and in $\mathrm{dB}$ (normalized to maximum PSD inside the channel) and denoted as Adjacent Channel Leakage Ratio (ACLR) (also called output transmit mask). For example, the output transmit mask of the IEEE802.11a standard is shown in Fig. $1.7(\mathrm{~b})$. 


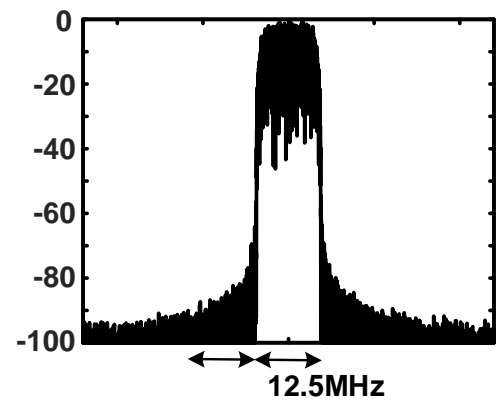

(a)

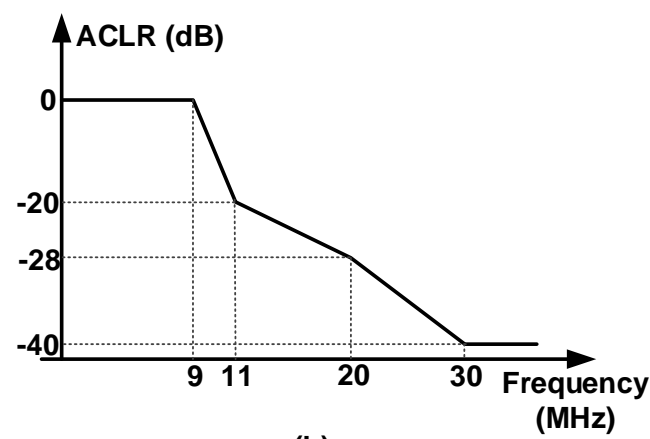

(b)

Figure 1.7: (a) PSD of a 64 QAM amplitude modulated signal with $10 \mathrm{MSymb}$./sec symbol rate (60Mbit/sec bit rate) and $12.5 \mathrm{MHz}$ bandwidth (sampling frequency is 8GS/sec). (b) the IEEE802.11a ACLR mask.

\subsection{Error Vector Magnitude}

For the BPSK modulation scheme, it was discussed that any nonidealities at the transmitters or recievers make the constellation points deviate from their ideal locations. The Error Vector Magnitude (EVM) is a measure that shows the deviation of constellation points of the modulated signal with respect to the ideal constellation points and is normally reported both in percentage and in $\mathrm{dB}$ (Fig. 1.8). In practice, to obtain the EVM, a constellation diagram based on a large number of detected samples (say $N_{s}$ ) is constructed and the error vectors $e_{i}, i=1,2, \ldots, N_{s}$ (drawn between each measured point and its ideal position) are obtained. The EVM is defined as the rms magnitude of the error vectors normalized to the signal rms voltage $V_{r m s}$ [4]

$$
\operatorname{EVM}(\%)=100 \times \frac{\sqrt{\frac{\sum_{i} e_{i}^{2}}{N_{s}}}}{V_{r m s}}
$$

The requirement on the EVM is normally given in the regulation documents of the target application.

\subsection{Peak to Average Power Ratio (PAPR) of mod- ulated signals}

PAPR is the ratio of peak power to the average power of a signal and is expressed in units of $\mathrm{dB}$. High order amplitude modulated signals, such as 64 QAM, exhibits 


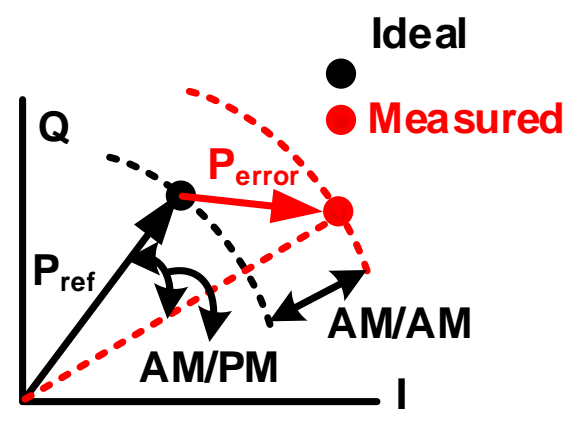

Figure 1.8: Illustration of EVM and the effect of AM/AM and AM/PM distortions on the constellation points.

high PAPR, i.e., the signal can have peaks that are much larger than the signal Root Mean Square (RMS) value. For transmission of such peaky signals, the PAs need to be designed to provide the peak power but are mainly operating at (the much lower) average output power. In the next chapter we will see that pushing PAs to operate at lower than peak power (in back off) results in a lower PA efficiency compared to that at peak output power.

As an example, a simulated Probability Density Function (PDF) of a 64 QAM signal (with raised-cosine pulse shaping, $12.5 \mathrm{MHz} \mathrm{BW}$ and $\alpha_{r}=0.25$ ) is shown in Fig. 1.9 as a function of power back-off (normalized to peak power). The average power of the signal is $7 \mathrm{~dB}$ below its peak level, i.e. PAPR $=7 \mathrm{~dB}$. PAPRs for different QAM levels (with raised-cosine pulse shaping, $12.5 \mathrm{MHz} \mathrm{BW}$ and $\alpha_{r}=0.25$ ) are given in Table 1.1. It can be seen that, by increasing the QAM level to achieve higher communication speeds, the PAPR increases that can result in a (potentially) poor efficiency for the PA.

Table 1.1: PAPR for different QAM levels.

\begin{tabular}{|l|l|l|l|l|}
\hline QAM level & 4 & 16 & 64 & 156 \\
\hline PAPR (dB) & 4.9 & 6.1 & 7 & 7.6 \\
\hline
\end{tabular}




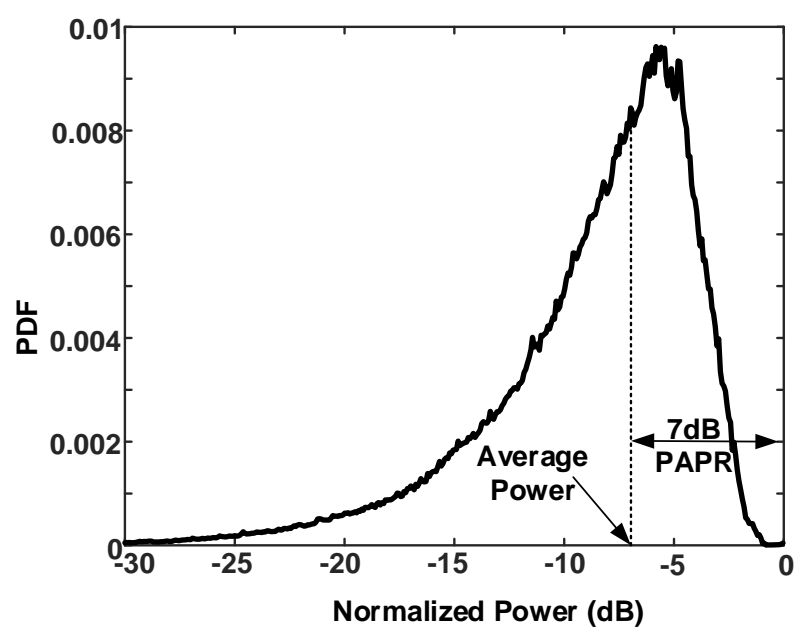

Figure 1.9: Simulated PDF of 64 QAM signal with raised-cosine pulse shaping and $\alpha_{r}=0.25$.

\subsection{Thesis organization}

The thesis work aims at high performance and reliable CMOS PAs. Various PA classes are briefly reviewed in chapter 2. The chapter also provides the rationale behind our choice of class E PAs as the PA configuration of choice and outphasing for linearization of this class of PA.

Antenna load-mismatch is a major external effect that can impact PAs performance and reliability. In chapter 3 we briefly discuss load-pull study of linear class A PAs as an introduction into class E PA load-pulling. Also transistor break-down mechanisms under higher than nominal voltage and current excursions are reviewed.

Traditionally, isolators/circulators were used to isolate the PA from mismatch at the antenna. However, their associated loss makes them unsuitable for use in highefficiency PA systems. To remove the necessity for these bulky components, firstly, a clear picture must be provided on the effect of load-mismatch on the performance and reliability of class E PAs. Chapter 4 presents load-pull analysis of class E PAs from mathematical perspective, validated by experimental results.

To be able to use class-E PAs to amplify amplitude modulated signals, the outphasing technique is used. However, load modulation that is being used to provide high efficiency both at maximum output power and at power back-off, is also a source of load-mismatch that can potentially compromise the reliability of such PAs when used in outphasing configurations. Furthermore, it is shown that the traditional mod- 
eling of PAs as ideal voltage sources cannot be employed to study outphasing systems using class E PAs as branch amplifiers [10].

As a next step, this thesis extends the study of chapter 4 in chapter 5 to provide a theoretical model for outphasing class-E PAs (OEPAs) to study performance aspects such as efficiency, operational bandwidth and output power dynamic range as well as reliability aspects such as maximum switch voltage. This chapter also presents a novel technique to improve the performance and reliability of such PAs under nominal antenna load conditions.

The general model of OEPAs, developed in chapter 5, is employed in chapter 6 to provide a model for the linearity of OEPAs. The provided linearity model was then successfully employed to pre-distort the input signal to the PA without being required to (conventionally) characterize the $\mathrm{AM} / \mathrm{AM}$ and $\mathrm{AM} / \mathrm{PM}$ distortions.

The model of the class-E PAs under load-mismatch, provided in chapter 4 , is employed in chapter 7 to design a self-protective class-E PAs under heavy load-mismatch conditions. The experimental verifications demonstrate that the PA can maintain its reliability under heavy load-mismatch condition with VSWRs up to 19:1, while the output power and the efficiency are not considerably affected. Chapters 4-7 are reformatted publications [11-14], respectively. Finally, chapter 8 summarizes this thesis, with recommendations on directions for future research. 


\section{Bibliography}

[1] S. Cripps, RF Power Amplifiers for Wireless Communications, 2nd edition, Artech House, 2006.

[2] T. S. Rappaport, Wireless Communications, Principles and Practice, New Jersey: Prentice Hall, 1996.

[3] L. W. Couch, Digital and Analog Communication Systems, Fourth Edition, New York: Macmillan Co., 1993.

[4] B. Razavi, Design of Analog CMOS Integrated Circuits. McGraw-Hill; 2001.

[5] M. P. van der Heijden, M. Acar, J. S. Vromans and D. A. Calvillo-Cortes, "A $19 \mathrm{~W}$ high-efficiency wide-band CMOS-GaN class-E Chireix RF outphasing power amplifier," 2011 IEEE MTT-S International Microwave Symposium, Baltimore, MD, 2011, pp. 1-4.

[6] K. Boyle and M. Leitner, "Mobile phone antenna impedance variations with realusers and phantoms", Proc. iWAT, pp. 420-423, Hong Kong, 2011.

[7] S. M. Bowers, K. Sengupta, K. Dasgupta, B. D. Parker and A. Hajimiri, "Integrated Self-Healing for mm-Wave Power Amplifiers," in IEEE Transactions on Microwave Theory and Techniques, vol. 61, no. 3, pp. 1301-1315, March 2013.

[8] D. M. Pozar, Microwave Engineering. 4th edition, Wiley; 2001.

[9] P. Smith, Electronic Applications of the Smith Chart, Noble Publishing Corporation, 2000

[10] R. Zhang, M. Acar, M. P. van der Heijden, M. Apostolidou and D. M. W. Leenaerts, "Generalized Semi-Analytical Design Methodology of Class-E Outphasing Power Amplifier," in IEEE Transactions on Circuits and Systems I: Regular Papers, vol. 61, no. 10, pp. 2951-2960, Oct. 2014. 
[11] A. Ghahremani, A. Annema and B. Nauta, "Load Mismatch Sensitivity of ClassE Power Amplifiers," in IEEE Transactions on Microwave Theory and Techniques, vol. 67, no. 1, pp. 216-230, Jan. 2019.

[12] A. Ghahremani, A. Annema and B. Nauta, "Outphasing Class-E Power Amplifiers: From Theory to Back-Off Efficiency Improvement," in IEEE Journal of Solid-State Circuits, vol. 53, no. 5, pp. 1374-1386, May 2018.

[13] A. Ghahremani, A. Annema and B. Nauta, " A +20 dBm Highly Efficient Linear Outphasing Class-E PA Without AM/AM and AM/PM Characterization Requirements," in IEEE Transactions on Circuits and Systems II: Express Briefs, vol. 66, no. 7, pp. 1149-1153, July 2019.

[14] J. Ponte, A. Ghahremani, M. Huiskamp, A. Annema and B. Nauta, "Augmentation of Class-E PA Reliability under Load Mismatch Conditions," 2018 25th IEEE International Conference on Electronics, Circuits and Systems (ICECS), Bordeaux, 2018, pp. 33-36. 


\section{Chapter 2}

\section{Power Amplifiers: Methods of Amplification}

Power amplifiers can be grouped into two main categories. The first category is that of the classically linear amplifiers that include class A, B, AB and C. The second category is that of the newer so-called switch-mode amplifiers that include class $\mathrm{D}, \mathrm{E}$ etc. PAs, normally, provide the maximum efficiency at maximum output power and the efficiency declines for reduced output power. Several topologies have been proposed to maintain high efficiency at power back-off such as Doherty [1], outphasing [2] etc. In this chapter we briefly review the PA classes and different proposed structures to maintain high back-off efficiency. For more information we refer to [3,4].

This chapter also provides the rationale behind our choice of class E PAs as the PA configuration of choice and outphasing for linearization of this class of PA.

\subsubsection{Linear PAs}

A simplified schematic of a linear PA is shown in Fig. 2.1. The PA consists of a transistor operating in class $\mathrm{A}, \mathrm{AB}, \mathrm{B}$ or $\mathrm{C}$ mode, depending on the fraction of time that the transistor conducts current (denoted by $\alpha \in[0,2 \pi]$ in Fig. 2.1). The transistor is biased to supply voltage $V_{D D}$ through an RF choke and a harmonic trap may be necessary (for classes $\mathrm{AB}, \mathrm{B}$ and $\mathrm{C}$ ) at the transistor output to provide a low impedance path for the harmonics of the drain current. For this purpose, a high-Q tank can be used in parallel with the PA load. For a suitable input power level, maximum power is delivered to the load when the drain current excursion is $\left[0, I_{\text {Max }}\right]$ (for class A PA $I_{M a x}$ is two times the PA bias current) and the drain (collector) voltage excursion (with $180^{\circ}$ phase shift with respect to drain current) is $\left[0,2 V_{D D}\right]$.

The output voltage phasor is related to the fundamental harmonic of the drain 

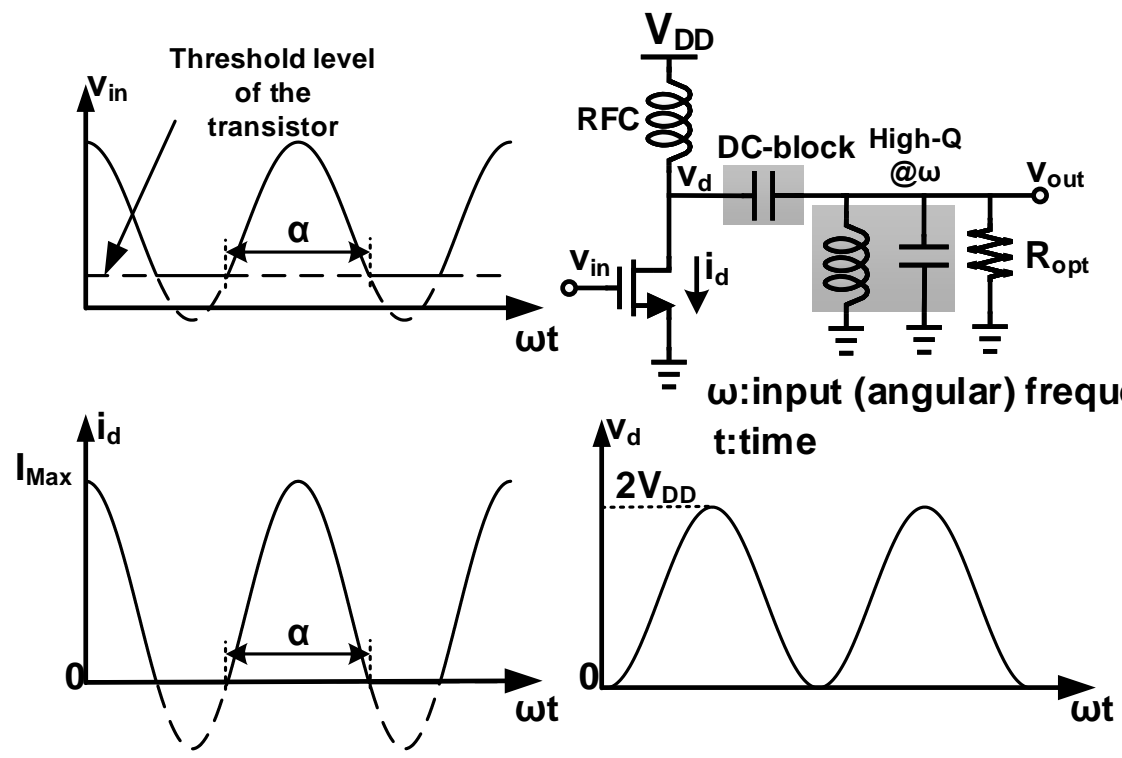

Figure 2.1: A simplified schematic of a linear PA and corresponding waveforms for maximum output pwoer. The high-Q filter acts as a short circuit at the harmonics of the input frequency.

current $i_{d}$ as $V_{\text {out }}=R_{\text {opt }} I_{d 1}$ where [3]

$$
I_{d 1}=\frac{I_{\text {Max }}}{2 \pi} \frac{\alpha-\sin (\alpha)}{1-\cos (\alpha / 2)}
$$

is the fundamental harmonic. The optimum load $R_{\text {opt }}$ yielding maximum output power is obtained as

$$
R_{o p t}=V_{D D} / I_{d 1}
$$

and for an ideal class A PA $(\alpha=2 \pi)$ we have $I_{d 1}=I_{M a x} / 2=I_{\text {Bias }}$, where $I_{\text {Bias }}$ is the bias current of the PA in class A configuration.

\section{Drain Efficiency and Power Utilization Factor (PUF)}

To find the drain efficiency (DE) we calculate the power delivered by the supply voltage as $P_{s}=V_{D D} \cdot\left\langle i_{d}(t)>(<\cdot>\right.$ stands for averaging) where

$$
<i_{d}(t)>=\frac{I_{\text {Max }}}{2 \pi} \frac{2 \sin (\alpha / 2)-\alpha \cos (\alpha / 2)}{1-\cos (\alpha / 2)}
$$


Then the DE of the PA for conduction angle $\alpha \in[0,2 \pi]$ is

$$
D E=\frac{1}{2} \frac{\alpha-\sin (\alpha)}{2 \sin (\alpha / 2)-\alpha \cos (\alpha / 2)}
$$

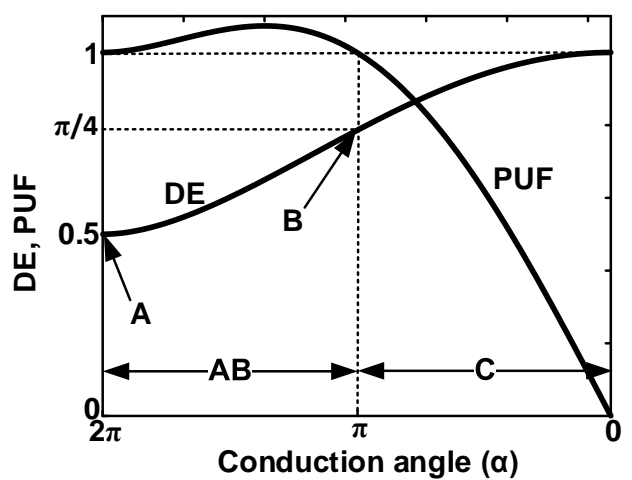

(a)

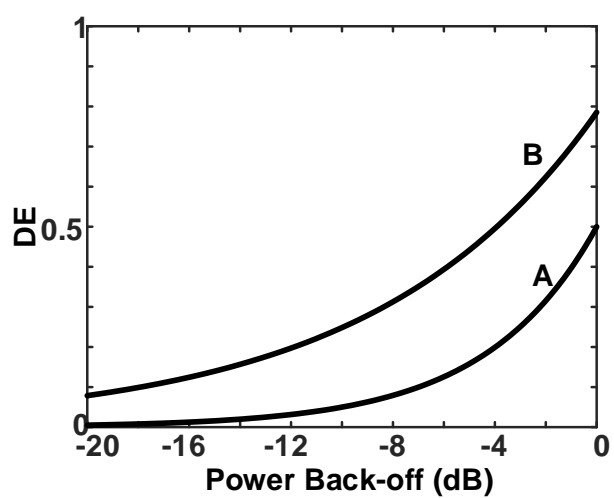

(b)

Figure 2.2: (a) RF power relative to Class A (PUF) and DE as a function of conduction angle. (b) Efficiency of class A and class B PAs versus power back-off.

The power-output capability of a PA is defined as the ratio of RF power delivered by a transistor normalized to $I_{M a x} V_{M a x}$ where $V_{M a x}$ is the maximum transistor voltage excursion. The power utilization factor (PUF) is defined as the power-output capability normalized to that if the PA was used in class A regime with the same $I_{\text {Max }}$ and $V_{\text {Max }}$. Then, from (2.1) and (2.2) and with $V_{M a x}=2 V_{D D}$, the PUF is derived as [3]

$$
\mathrm{PUF}=\frac{1}{\pi} \frac{\alpha-\sin (\alpha)}{1-\cos (\alpha / 2)}
$$

For class A, AB, B and C PAs we have $\alpha=2 \pi, \pi<\alpha<2 \pi, \alpha=\pi$ and $0<\alpha<\pi$, respectively. PAs DE and PUF are shown in Fig. 2.2(a) as a function of conduction angle $\alpha$. Class A PAs have (at best) $50 \%$ efficiency with PUF $=1$. Class B PAs can enhance efficiency to $\pi / 4 \approx 79 \%$ without any PUF degradation. However, class C PAs enhance the efficiency further at the cost of output power.

Note that the derived and plotted DE in Fig. 2.2(a) is at maximum output power. The DE of all classes of operation reduces when reducing the input drive (and hence the output power) at constant $V_{D D}$ (and constant $I_{B}$ for class A PAs). For example for class A and B PAs, the efficiency as a function output voltage amplitude 
$0<V_{\text {out }}<V_{D D}$ can be written as

$$
\begin{array}{r}
D E_{A}\left(V_{\text {out }}\right)=\frac{1}{2}\left(\frac{V_{\text {out }}}{V_{D D}}\right)^{2} \\
D E_{B}\left(V_{\text {out }}\right)=\frac{\pi}{2} \frac{V_{\text {out }}}{V_{D D}}
\end{array}
$$

The DE as a function of power back-off (defined as $\left.\mathrm{BF}=10 \log \left(P_{\text {out }} / \max \left(P_{\text {out }}\right)\right)\right)$ is shown in Fig. 2.2(b). It can be seen that at 10dB back-off the DE of class A PAs drops to (almost) $5 \%$ while class B PAs provide (at best) $25 \% \mathrm{DE}$.

\section{Non-ideal effects and PAE}

The calculated DE numbers are the maximum achievable numbers for the relevant classes. In practice, there is some drive power needed for proper operation of the PAs. As a result the overall efficiency (or PAE) numbers of the PAs are lower than DE numbers. For linear PAs and at a constant output power, the required drive power increases by reducing the conduction angle. Therefore, at $\mathrm{GHz}$ frequencies where the device gain is limitted, class C PAs are less attractive due to poor overall efficiency (i.e. PAE) $[3,5]$.

Moreover, second order effects such as knee voltage, drain parasitic resistance and capacitance and losses of the passive components impact DE and PAE numbers considerably. For example, in [6], a class AB PA is implemented in a standard 90nm CMOS process with operational BW 5.2 to $13 \mathrm{GHz}$. The measured PAE at $25.2 \mathrm{dBm}$ maximum output power is $21.6 \%$. By limiting the operational $\mathrm{BW}$, a better efficiency could be achieved. With proper termination of the second harmonic of the drain current, in [7], the PAE is improved to $28.4 \%$ at maximum output power.

\section{Back-off efficiency improvement techniques}

To improve the back-off efficiency of linear PAs, several techniques have been proposed. The PA supply voltage can be reduced in power back-off; PAs with continuous and discrete supply modulation are denoted as Class G and Class H, respectively. In [8], two voltage supplies are employed to improve back-off efficiency of a class B PA. At $1.8 \mathrm{GHz}$ and $27.2 \mathrm{dBm}$ maximum output power, the measured $\mathrm{PAE}$ is $30 \%$ and when the supply is switched to $V_{D D} / 2, \times 2 \mathrm{PAE}$ improvement at $6 \mathrm{~dB}$ power back-off was measured.

Another proposed technique in literature utilizes impedance level tuning which can be performed by a tunable matching network [9]. In using this technique the power back-off is achieved by reducing the input drive power while the output voltage amplitude is kept constant by means of tuning the load impedance to higher values (so called load trajectory manipulation or LTM in short). Due to limitations in the 
load tuning range, this technique can be combined with supply modulation technique (of class G PAs) to improve the efficiency of e.g. a class B PA at deep power backoff. In [9] this technique has been employed; the PA achieves $39 \% \mathrm{DE}$ at $+24.6 \mathrm{dBm}$ maximum output power level from a $2.8 \mathrm{~V} V_{D D}$ at $2.4 \mathrm{GHz}$. Employing the proposed technique, at $V_{D D}=1.55 \mathrm{~V}$, at $12 \mathrm{~dB}$ power back-off $\times 2.2$ efficiency enhancement was measured; $21.8 \%$ DE. Other techniques to modulate the load impedance include Doherty and outphasing systems, which will be discussed in detail later in this chapter.

\subsubsection{Class J PAs}

True class B operation necessitates a short circuit (for the output to ground) at the harmonics of the operating frequency ${ }^{1}$. As long as the impedance $X_{c}$ of the device output capacitance $C$ is small enough $\left(X_{c} \leq R_{o p t}\right)$, the output capacitance can resemble the short circuit for the harmonics and close to classical class B efficiency can be achieved. However, some devices such as GaAs transistors exhibit a small output capacitance. Producing a short circuit at the harmonics for these devices can be challenging at $\mathrm{GHz}$ frequencies due to e.g. package parasitics.

In class J PAs, a current profile similar to class B PAs (with $\alpha=\pi$ conduction angle) is assumed. The output capacitance $C$, shown in Fig. 2.3, does not act as a short circuit, rather providing an impedance $X_{c}(n f)$ at the $n^{\text {th }}$ harmonic. A filter is used at the output to filter out the harmonic content of the load and a complex load is required (compared to real load needed for class B PAs) to achieve efficiencies comparable to those of class B PAs.

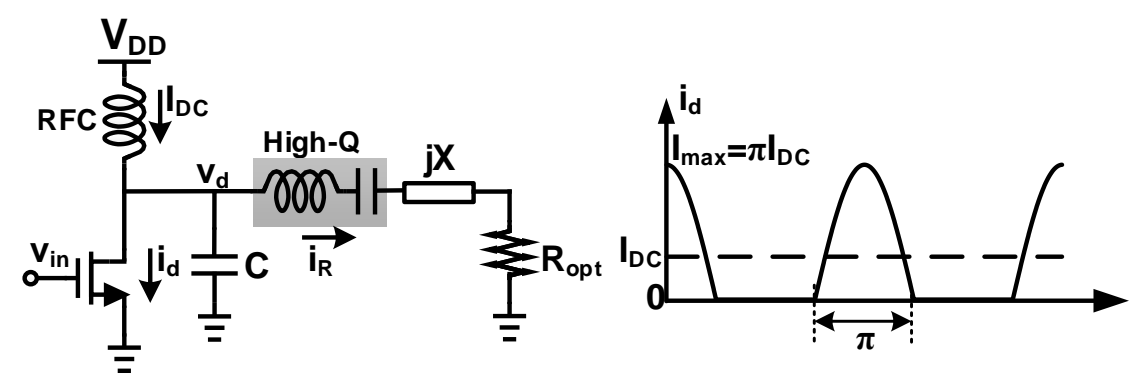

Figure 2.3: Class J PA schematic and transistor current waveform.

Class J PAs are studied in [3] in detail. It is shown that for $\alpha=\pi$, with a load with a proper imaginary part $j X$, the maximum efficiency of class B PA (i.e. $\eta=\pi / 4$ ) can

\footnotetext{
${ }^{1}$ True class B operation can also be achieved by employing classical push-pull structures, however, this option is not always available.
} 
be achieved for $X_{c}$ s up to about $2.5 R_{\text {opt }}$. However, this comes at the cost of a higher $V_{\text {Max }}$. By increasing the conduction angle, the maximum efficiency of the class $\mathrm{AB}$ PA with a similar conduction angle is achieved at a higher $X_{c}$ to $R_{\text {opt }}$ ratio. In [10], a class J PA employing LTM to provide the optimum complex impedance at power back-off and including power supply modulation is designed at $2.08 \mathrm{GHz}$ using a GaN HEMT transistor with $38 \mathrm{dBm}$ output power and above $45 \%$ DE over an $8 \mathrm{~dB}$ power back-off range.

\subsubsection{Class F PAs}

Considering the drain voltage and current waveforms of a class B PA $(\alpha=\pi)$ in Fig. 2.1, the power loss (which drops the DE from 100\% to $79 \%$ ) is due to non-zero time-overlap between the voltage and current in the transition. A method to reduce this overlap is to let the drain voltage converge to a square-like waveform by adding some odd harmonics to the drain voltage with appropriate amplitudes and phases. Letting a 3rd order harmonic of the drain voltage appear with relative amplitude $1 / 6$, PA DE can be improved from $79 \%$ for class B to $90.7 \%$ [3]. Furthermore, this also increases the PUF from 1 for class B PAs to 1.16.

Ideally, the drain current waveform contains only the fundamental and even harmonics and to generate odd harmonics, designers rely on the non-linearities and saturation in the transistor. Furthermore, to obtain the proper amplitude for the third order harmonic of the drain voltage, a proper impedance should be provided for the corresponding drain current harmonic which makes the output filter for class F PAs more complex than other PAs and as a result less attractive for integrated solutions.

Proper impedance termination at odd (higher than 3rd) harmonics, efficiency and PUF can be further improved at the cost of a more complex output filter. Ultimately, by using an infinite number of harmonics, efficiency can be increased to $100 \%$ [11].

\subsubsection{Class D PAs}

A class D PA, in its conventional form, consists of two switches to generate a square drain waveform $v_{d}(t)$, shown in Fig. 2.4, and a high-Q series filter that passes the fundamental harmonic to the load, resulting in an output power $P_{\text {out }}=\left(2 / \pi^{2}\right) V_{D D}^{2} / R_{\text {opt }}$. Assuming ideal passive components and ideal switches with a zero switch on-resistance and neglecting the power loss associated with charging (to $V_{D D}$ ) and discharging (to $0 \mathrm{~V}$ ) of the parasitic drain capacitance $C$, class D PAs provide $100 \%$ efficiency. Maximum voltage and current excursions across and through the transistors in a class D configuration are $V_{M a x}=V_{D D}$ and $I_{M a x}=(2 / \pi) V_{D D} / R_{o p t}$ which result in a power-output capability $1 /(\pi)$, which is the highest of any PA class $[3,5]$.

To perform amplitude modulation, different techniques can be employed. Pulse 


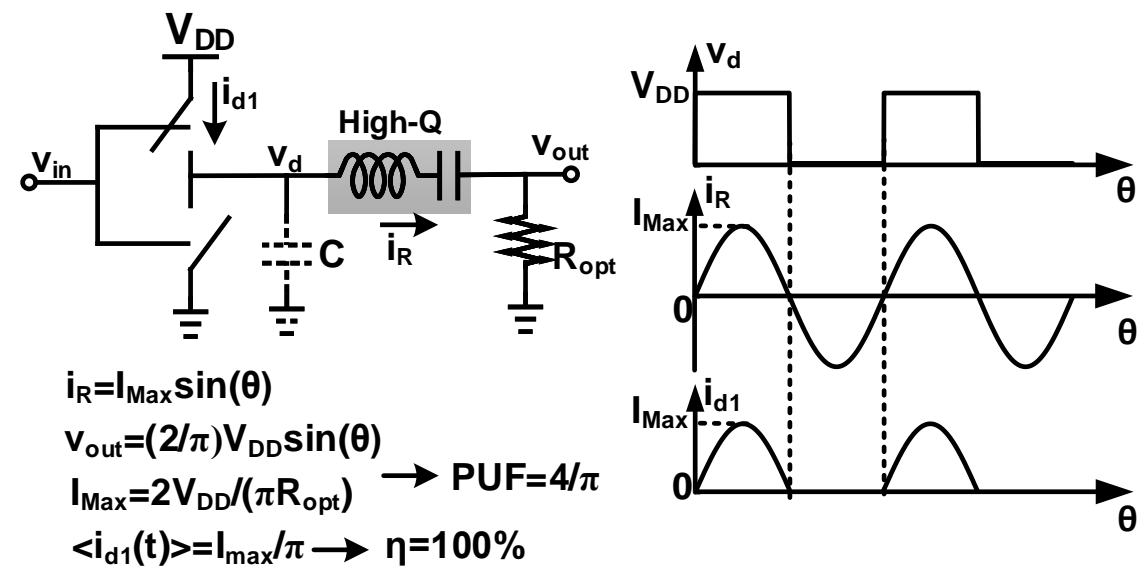

Figure 2.4: Class D PA schematic and waveforms.

width modulation (PWM), suitable for audio applications [12], can be used to design high power multi-watt PAs with (measured) efficiencies as high as $90 \%$ at maximum output power. The amplitude of output voltage of a class D PA is proportional to the supply voltage $V_{D D}$; for amplitude modulation, one can modulate $V_{D D}$. This technique is known as envelope elimination and restoration (EER) which is also denoted as Polar technique (this will be discussed later in this chapter). Employing this technique, in [13], a class D PA is implemented in a standard 130nm CMOS process at $1 \mathrm{GHz}$ providing $32 \% \mathrm{DE}$ at $12 \mathrm{dBm}$ maximum output power.

Another technique to apply amplitude modulation to class D PAs is to use the so-called switch capacitor PA (SCPA) structure, shown in Fig. 2.5. The switches and the filter capacitance $C_{0}$ are divided into $\mathrm{N}$ equal size unit elements being controlled by a control word with $\mathrm{N}$ bits. If a control bit $b_{i}(\mathrm{i}=1,2, \ldots, \mathrm{N})$ is 1 , the $\mathrm{i}^{\text {th }}$ class $\mathrm{D}$ unit is in on-state and switches the left plate of $C_{u i}$ between 0 and $V_{D D}$. For $b_{i}=0$, the corresponding unit PA is in off-state and capacitor $C_{u i}$ is connected to ground. The output voltage amplitude is [14]

$$
V_{\text {out }}=\frac{\sum_{i} b_{i} C_{u i}}{C_{0}} V_{D D}
$$

For the configuration of Fig. 2.5, $\mathrm{N}$ different levels of output amplitude can be realized. The work in [14] is implemented in a standard 90nm CMOS process and has reported a measured PAE of $45 \%$ at $25.2 \mathrm{dBm}$ output power at $2.4 \mathrm{GHz}$. To improve the back-off efficiency a multi-level supply voltage (Class G SCPA) is employed in [15] and yielding an improvement from $27 \%$ (reported in [14]) to $33 \%$ in PAE when 


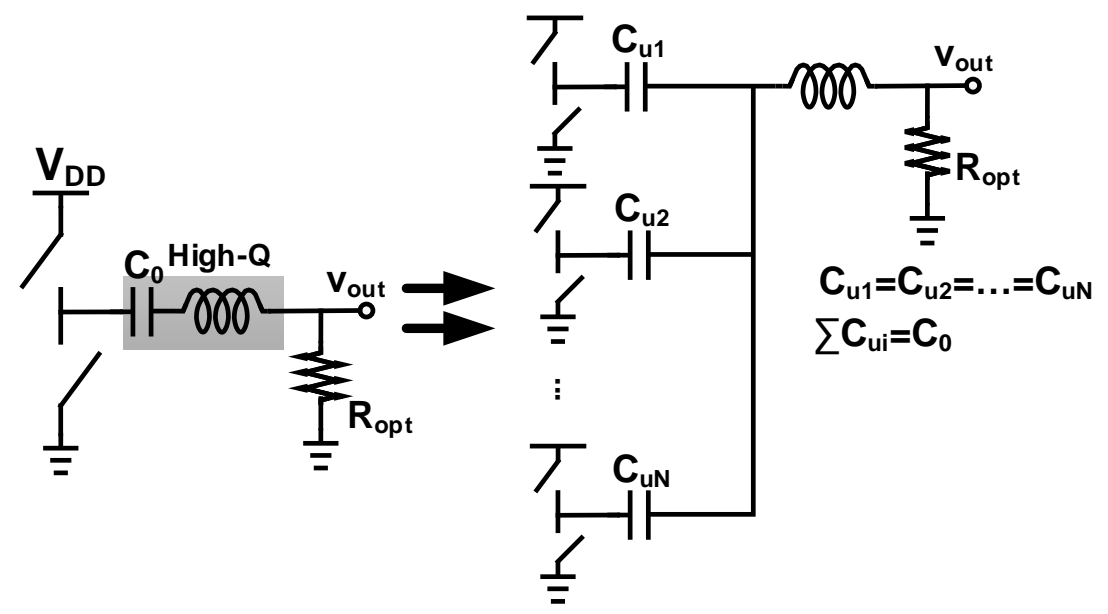

Figure 2.5: Simplified diagram of an ideal single-ended SCPA.

amplifying 802.11g 64-QAM OFDM signals.

\subsubsection{Class E PAs}

A simplified schematic of a class E PA is shown in Fig. 2.6. A transistor is employed as a switch controlled by a square-shaped driving waveform. When the switch is the on-state, ideally, $v_{c}=0^{2}$ and the inductor $L$ is charged by supply voltage $V_{D D}$. When the switch goes into off-state, the energy of the inductor propagates toward the load. By proper selection of the component values $L$ and $C$ and the load impedance $R_{o p t}+j X$, the drain voltage reaches zero and has zero slope when the transistor turns on. This eliminates the associated loss with charging the drain capacitance (that impacts class D PAs efficiency). Ideally, a class E PA provides $100 \%$ efficiency and a PUF of more than 0.8 [16]. This class of PAs is the subject of this thesis and we will further discuss this class in chapter 4 .

\subsubsection{Doherty structure}

The Doherty amplifier, proposed in [1], is a technique to improve the back-off efficiency of PAs by means of load modulation. The structure, shown in Fig. 2.7(a), consists of two amplifiers; one main (typically A, B or AB) and one auxiliary (typically B or C). At maximum output power and at power back-offs up to (typically) $6 \mathrm{~dB}$ both

\footnotetext{
${ }^{2}$ For consistency with the next chapters, for class E PAs, we use $v_{c}$ as the drain/switch/capacitor voltage.
} 

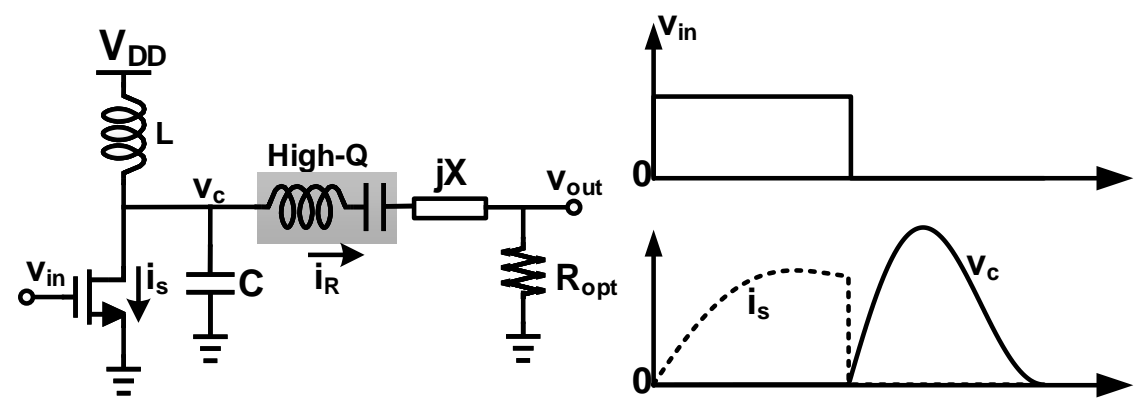

Figure 2.6: Class E PA schematic and waveforms.

amplifiers contribute to the output power, while for back-off levels of more than $6 \mathrm{~dB}$, the auxiliary amplifier shuts down.

For a simplified Doherty structure of Fig. 2.7(b), let's assume class B operation. A high-Q LC tank was assumed in parallel with the transistors to shunt all the drain current harmonics to ground. Then, the output currents are the first harmonic of the drain currents and the output voltages are the DC-blocked drain voltages. The output current phasors (as a function of the input amplitude) can be written as

$$
\begin{aligned}
I_{\text {out } 1} & =\frac{I_{\text {Max }}}{2} \frac{\left|V_{\text {in }}\right|}{V_{\text {in }, M}} \\
I_{\text {out } 2} & =-j \frac{I_{\text {Max }}}{2}\left(2 \frac{\left|V_{\text {in }}\right|}{V_{\text {in }, M}}-1\right) ; 0.5<\frac{\left|V_{\text {in }}\right|}{V_{\text {in }, M}}<1 \\
& =0 ; \frac{\left|V_{\text {in }}\right|}{V_{\text {in }, M}}<0.5
\end{aligned}
$$

where $V_{i n, M}$ is the maximum input drive (0dB back-off) and $\left|V_{i n}\right|=V_{i n, M} / 2$ corresponds to the $6 \mathrm{~dB}$ power back-off. The definition of $I_{M a x}$ was given in Fig. 2.1 and the output current (waveform) amplitudes are shown in Fig. 2.7(c). For the quarter wave-length transmission line with characteristic impedance $Z_{0}$, in Fig. 2.7(b),

$$
\begin{aligned}
V_{\text {out } 1} & =j Z_{0} I_{t} \\
V_{\text {out } 2} & =V_{\text {out }}=-j Z_{0} I_{\text {out } 1} \\
V_{\text {out }} & =R_{\text {opt }}\left(I_{\text {out } 2}+I_{t}\right) / 2
\end{aligned}
$$

Rearranging these equations, for $0.5<\frac{\left|V_{i n}\right|}{V_{i n, M}}<1$, it can be found that

$$
V_{\text {out } 1}=2 \frac{Z_{0}^{2}}{R_{\text {opt }}} I_{\text {out } 1}-j Z_{0} I_{\text {out } 2}
$$




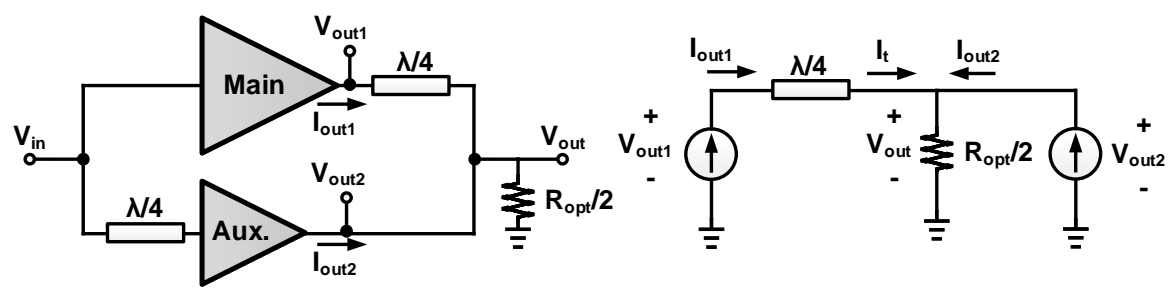

(a)

(b)
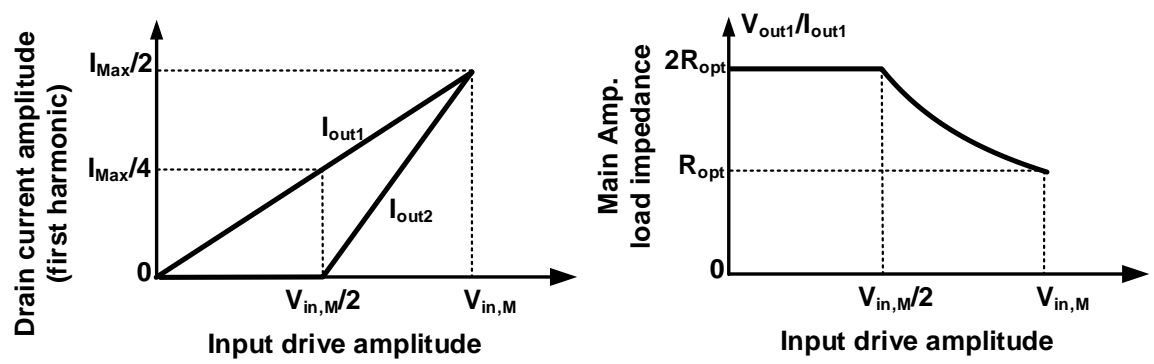

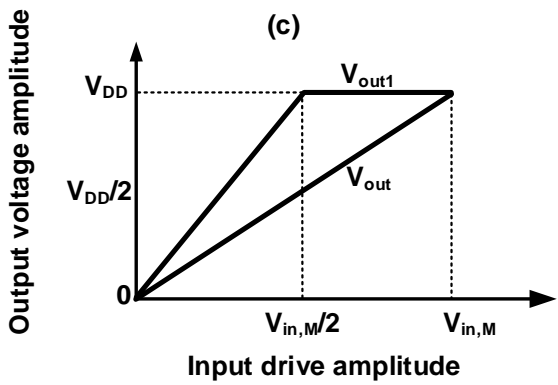

(e)

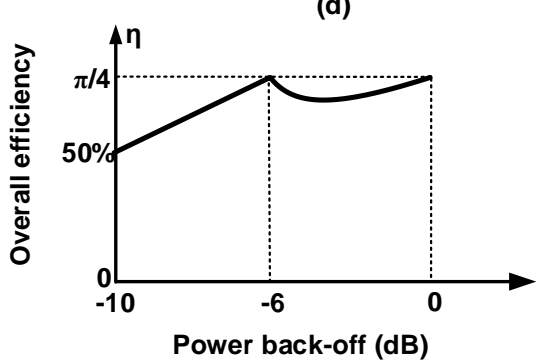

(f)

Figure 2.7: Doherty PA (a) basic configuration, (b) simplified schematic. For class B main and auxiliary amplifiers (c) output current, (d) impedance seen by the main amplifier, (e) output voltage versus input drive. (f) overall efficiency versus power back-off when main and auxiliary amplifiers are operating in a class B configuration. 
For $Z_{0}=R_{o p t}$ it follows from (2.9) and (2.10) that the output voltage amplitude of the main amplifier is constant, $V_{\text {out } 1}=R_{\text {opt }} \frac{I_{M a x}}{2}$, for $6 \mathrm{~dB}$ of output power range. Recalling (2.7)/(2.6), for a class B/A PA with a constant supply voltage, the efficiency can be kept as high as the efficiency at maximum output power level for $6 \mathrm{~dB}$ of output power range.

The load impedance, seen by the main amplifier for $0.5<\frac{\left|V_{i n}\right|}{V_{i n, M}}<1$ can be obtained from (2.9) and (2.14) as

$$
Z_{1}=\frac{V_{\text {out } 1}}{I_{\text {out } 1}}=2 R_{\text {opt }}\left(\frac{2 \frac{\left|V_{\text {in }}\right|}{V_{\text {in }, M}}-1}{\frac{\left|V_{\text {in }}\right|}{V_{\text {in }, M}}}\right) ; 0.5<\frac{\left|V_{\text {in }}\right|}{V_{\text {in }, M}}<1
$$

For higher back-off levels, the auxiliary amplifier is disabled and does not contribute to the output power. In this case, the main PA sees a constant load (no load modulation) $2 R_{\text {opt }} . Z_{1}$ as a function of input drive voltage amplitude is shown in Fig. 2.7(d). It can be seen that the Doherty structure modulates the load impedance seen by the main amplifier to keep a constant voltage amplitude over a $6 \mathrm{~dB}$ output power range to maintain high efficiency. For this back-off range, using (2.12) and (2.9), the output voltage phasor $V_{\text {out }}$ can be written as

$$
V_{\text {out }}=-j Z_{0} I_{\text {out } 1}=-j R_{\text {opt }} \frac{I_{\text {Max }}}{2} \frac{\left|V_{\text {in }}\right|}{V_{\text {in }, M}} ; 0.5<\frac{\left|V_{\text {in }}\right|}{V_{\text {in }, M}}<1
$$

which shows a linear function of input drive signal. For $\frac{\left|V_{i n}\right|}{V_{i n, M}}<0.5$, the auxiliary amplifier is disabled and the output voltage can be readily written as

$$
V_{\text {out }}=\frac{V_{\text {out } 1}}{2} ; \frac{\left|V_{\text {in }}\right|}{V_{\text {in }, M}}<0.5
$$

Output voltage amplitude $V_{\text {out }}$ and the output voltage amplitude of the main amplifier as a function of the input drive are shown in Fig. 2.7(e). The Doherty PA can be seen to exhibit linear behavior over the entirety of the shown input driving signal range.

The output voltage of the auxiliary amplifier as shown in Fig. 2.7(e) shows a linear decrease from its maximum value. Therefore, this amplifier cannot maintain its maximum efficiency over the $6 \mathrm{~dB}$ back-off range. However, by going into back-off, the contribution of the auxiliary amplifier to the output power reduces and therefore the impact on overall efficiency is modest [3]. Assuming class B operation for both the PAs, it can be shown that [3]

$$
\begin{aligned}
\eta & =\frac{\pi}{2} \frac{\left(\frac{V_{\text {in }}}{V_{\text {in } \max }}\right)^{2}}{3\left(\frac{V_{\text {in }}}{V_{\text {in }, \max }}\right)-1} 0.5<\frac{\left|V_{\text {in }}\right|}{V_{\text {in }, M}}<1 \\
& =\frac{\pi}{4} \frac{V_{\text {out } 1}}{V_{D D}} ; \frac{\left|V_{\text {in }}\right|}{V_{\text {in }, M}}<0.5
\end{aligned}
$$


Efficiency as a function of power back-off is shown in Fig. Fig. 2.7(f). It can be seen that the Doherty structure provides maximum efficiency both at maximum output power and at $6 \mathrm{~dB}$ back-off. The reduced efficiency at back-off levels between $0 \mathrm{~dB}$ and $6 \mathrm{~dB}$ is due to the auxiliary amplifier's reduced efficiency at back-off.

To further improve the back-off efficiency, the Doherty structure can be used with selectable two-level supply rail $\left(V_{D D}\right.$ and $\left.V_{D D} / 2\right)$; in [17] a saturated output power of $25.3 \mathrm{dBm}$ at $3.5 \mathrm{GHz}$ was measured with $30.4 \% / 25.3 \% / 17.4 \%$ PAE at $0 / 6 / 12 \mathrm{~dB}$ power back-off. The work in [17] is implemented in a 45nm CMOS SOI technology and replaced the transmission-lines in Fig. 2.7(a) with on-chip transformers.

\subsubsection{Envelope Elimination and Restoration (EER)}

EER was originally proposed by Kahn as a more efficient alternative to linear class AB PAs [18]. The system proposed by Kahn is shown in Fig. 2.8(a). In this system the amplitude information is eliminated by a limiter and therefore the PA can be employed in saturation (yielding high efficiency). For the saturated PA, the output voltage is proportional to its supply voltage and amplitude information can be provided by modulating the supply. An envelope detector is used to extract the amplitude information of the signal applied to the input of the limiter and then the amplitude information is restored at the PA output by using a supply modulator.

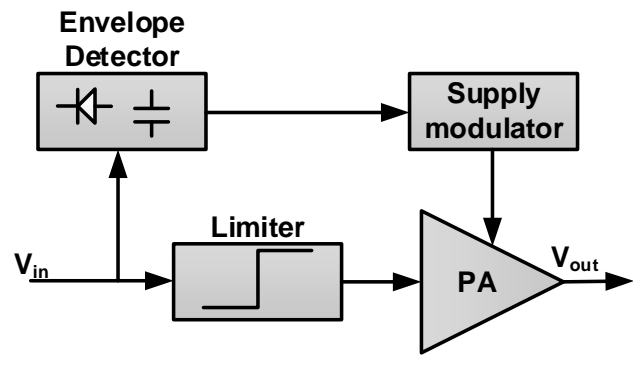

(a)

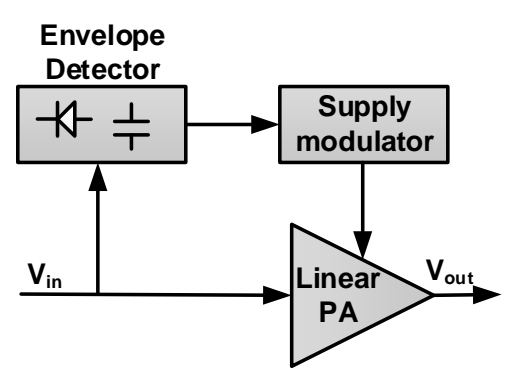

(b)

Figure 2.8: (a) Envelope Elimination and Restoration (EER) system. (b) Envelope Tracking (ET) PA system.

Nowadays, the envelope detector and limitter are omitted from the traditional EER system and the amplitude and phase information are directly and separately provided for the supply modulator and the PA, respectively, from a digital processing unit. The new structure is also denoted as polar PA. In EER systems, linear PAs (in saturation) or switched mode PAs (class D or E) can be used. Employing a class E 
PA in a standard 40nm CMOS, the work in [19] reported $67 \%$ DE and $45 \% \mathrm{PAE}$ at $2.6 \mathrm{GHz}$ at $17.2 \mathrm{dBm}$ maximum output power.

\subsubsection{Envelope Tracking (ET)}

In EER systems, amplitude modulation is provided by a supply modulator. This requires an accurate supply modulator and may have dynamic range limitations [3]. Another back-off efficiency enhancement technique (previously described as class G and H, under PA classifications) is Envelope Tracking (ET). In this system, shown in Fig. 2.8(b), a linear PA is used and the supply rail, $V_{D D}$, is always tuned (to slightly higher than maximum output voltage) to provide high back-off efficiency. Instead of a continuous $V_{D D}$ control, if only two levels are used, the PA can be denoted as operating in class $\mathrm{G}$.

\subsubsection{Outphasing}

The outphasing structure which is sometimes also denoted as Linear Amplification by Non-linear Components (LINC) [20-22], was proposed by Chireix in 1935 [2]. This structure, shown in Fig. 2.9(a), consists of a Signal Component Separator (SCS), two branch PAs always operating at a fixed drive power (and hence can be highly efficient) and a combiner.

The SCS converts input amplitude information, shown in Fig. 2.9(a) with $v_{i n}$, into phase difference $\Delta \theta$ between two constant envelop carriers $v_{i n 1}$ and $v_{i n 2}$;

$$
\begin{gathered}
v_{i n}=\left|V_{i n}\right| \cos \left(\omega_{0} t+\phi\right) \\
v_{i n 1}=\cos \left(\omega_{0} t+\phi+\frac{\Delta \theta}{2}\right) \\
v_{i n 2}=\cos \left(\omega_{0} t+\phi-\frac{\Delta \theta}{2}\right)
\end{gathered}
$$

where

$$
\Delta \theta=2 \cos ^{-1}\left(\frac{\left|V_{i n}\right|}{V_{i n, M}}\right)
$$

which is denoted as outphasing angle and $V_{i n, M}$ is the maximum amplitude of $v_{i n}$. The two branch PAs, which can be linear PAs in saturation or switched-mode nonlinear PAs (for high efficiency), amplify the two constant amplitude driving signals. The output signal at the combiner output can be described as

$$
\begin{aligned}
v_{\text {out }} & =G \cos \left(\omega_{0} t+\phi+\frac{\Delta \theta}{2}\right)+G \cos \left(\omega_{0} t+\phi-\frac{\Delta \theta}{2}\right) \\
& =2 G \cos \left(\frac{\Delta \theta}{2}\right) \cos \left(\omega_{0} t+\phi\right)=G \frac{\left|V_{\text {in }}\right|}{V_{\text {in }, M}} \cos \left(\omega_{0} t+\phi\right)
\end{aligned}
$$




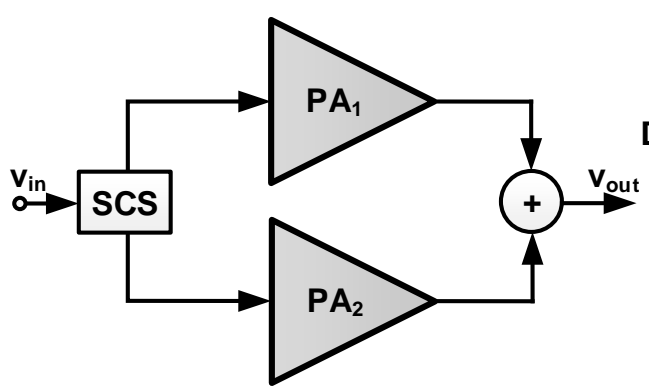

(a)

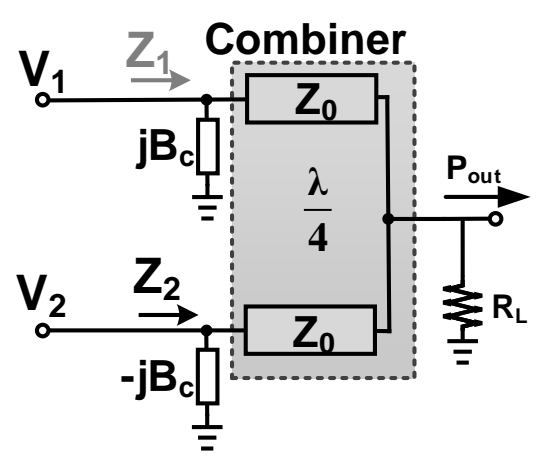

(c)

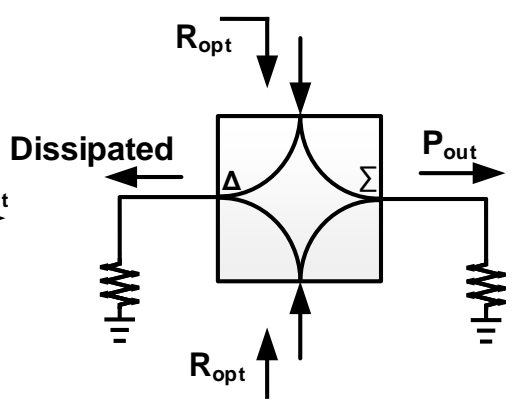

(b)

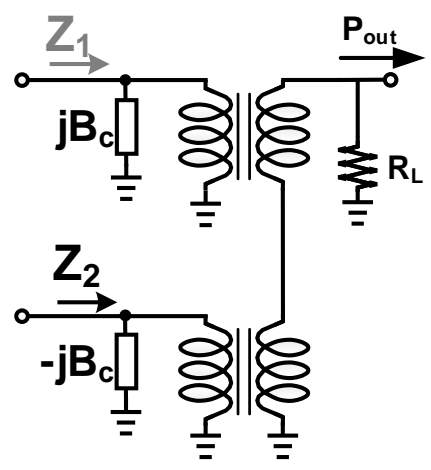

Figure 2.9: (a) Outphasing PA system. (b) isolating combiner. (c) Chireix transmission-line and transformer based non-isolating combiners.

where $G$ is the voltage gain of the branch PAs. Such a simplified linear operation, shown by (2.23), can be guaranteed by employing an isolating combiner such as a Wilkinson combiner [23], shown in Fig. 2.9(b). In doing so, the branch PAs always see a constant optimum load $R_{\text {opt }}$ over the entire outphasing angle range. Then, at back-off, the branch PAs still are operating at their maximum output power rating while only a fraction of the PA output power is delivered to load and the remainder is dissipated at the isolating port of the combiner. In [23] an energy recovery amplifier was proposed to convert the power absorbed by the isolating port back into DC power.

Chireix [2] proposed the use of a non-isolating power combiner with compensating reactive elements, shown in Fig. 2.9(c). The combiner can e.g. be based on transformers or transmission-lines. In doing so, due to the load-pulling between the two branch PAs, a load modulation can be presented to the branch PAs (similar to 
Doherty PAs) hence high efficiency can be obtained at deep power back-off. To analyze outphasing structures utilizing non-isolating combiners, traditionally, the branch PAs are modeled as ideal voltage sources with a phase difference $\Delta \theta$. Here we consider the transmission-line based combiner, however, similar results can be derived for transformer based combiners. Assuming the PAs output voltages $V_{1}=e^{\left(j \frac{\Delta \theta}{2}\right)}$ and $V_{2}=e^{\left(-j \frac{\Delta \theta}{2}\right)}$ and using even-odd mode analysis [24], it can be readily shown that

$$
\begin{gathered}
\frac{1}{Z_{1}}=\frac{2 R_{L}}{Z_{0}^{2}} \cos ^{2}\left(\frac{\Delta \theta}{2}\right)+j\left(-\frac{R_{L}}{Z_{0}^{2}} \sin (\Delta \theta)+B_{c}\right) \\
\frac{1}{Z_{2}}=\frac{2 R_{L}}{Z_{0}^{2}} \cos ^{2}\left(\frac{\Delta \theta}{2}\right)-j\left(-\frac{R_{L}}{Z_{0}^{2}} \sin (\Delta \theta)+B_{c}\right)
\end{gathered}
$$

where we assume $R_{L}$ is the load impedance connected to the combiner output, $Z_{0}$ is the transmission-line characteristic impedance and $\frac{Z_{0}^{2}}{2 R_{L}}=R_{o p t}$.

For $B_{c}=0$ (open circuit), at maximum output power (corresponding to maximum input amplitude or $\Delta \theta=0$ ) both branch PAs see an optimum load $Z_{1}=Z_{2}=R_{\text {opt }}$ and therefore provide the maximum efficiency, e.g. $79 \%$ in the case of class B branch PAs. However, by going into back-off $(0<\Delta \theta<\pi)$, due to non-zero imaginary part of PA loads, the efficiency drops significantly.

For $0<B_{c}<\frac{R_{L}}{Z_{0}^{2}}$, the imaginary power of the PA load impedance $Z_{1,2}$ is zero for two outphasing angles. At these angles, ideally, the maximum efficiency can be maintained. For $B_{c}=3 R_{\text {opt }}$, the (ideal) maximum efficiency $(79 \%)$ of an outphasing class B PA can be obtained at (almost) $0 \mathrm{~dB}$ and $10 \mathrm{~dB}$ back-off with more than $70 \%$ efficiency in the $0-10 \mathrm{~dB}$ back-off range [3]. In [25], by employing class E PAs (ideally) $100 \%$ efficiency at maximum output power as well as at $10 \mathrm{~dB}$ back-off was presented. Employing more compensation elements, in [26], the (ideal) 100\% efficiency was obtained at more back-off points. Further discussion of the outphasing technique is postponed to chapter 5 in this thesis.

An outphasing technique with class B PAs as branch amplifiers, implemented in 90nm CMOS technology in [29] and with an off-chip combiner at $0.9 \mathrm{GHz}$, reported a PAE of $56 \%$ at maximum $20 \mathrm{Bm}$ output power and a PAE of $20 \%$ at $12 \mathrm{~dB}$ power back-off. Using class D PAs in 45nm CMOS technology with an off-chip combiner at $2.4 \mathrm{GHz}$, in [30], at $25 \mathrm{dBm}$ maximum output power a DE (PAE) of $52 \%(45 \%)$ was demonstrated. At $12 \mathrm{~dB}$ power back-off the measured DE (PAE) is $12 \%(8 \%)$. Employing class E PAs as branch amplifiers in 40m CMOS technology with an on-chip combiner at $5.9 \mathrm{GHz}$, at maximum $22.2 \mathrm{dBm}$ output power a measured DE (PAE) of $49.2 \%(34.9 \%)$ was reported. At $12 \mathrm{~dB}$ back-off, the measured DE and PAE are less than $18 \%(13 \%)$.

Outphasing techniques can also be combined with other efficiency enhancement techniques to further improve the back-off efficiency; multi-level supply (class-G) was employed in [27] and the Doherty technique was combined with outphasing in [28]. 


\subsection{PA class selection}

In this thesis, our focus is on standard CMOS technologies due to their low cost and high level of integration. However, an advanced standard CMOS technology suffers from several issues including non-linear transconductance, temperature dependent mobility, problems arising from scaling such as process variations from chip to chip and model inaccuracies [32]. Switched mode PAs such as class D or E are less sensitive to these issues and provide higher efficiency among the PA classes.

Class D PAs provide the highest power-output capability. However, for the given supply and PA output loading their output power levels are quite low compared to class-E PAs [3]. For these reasons, class E PAs are the subject of this thesis. To be able to use these class E PAs with amplitude modulated signals, we use the outphasing technique due to its digital compatibility (phase-only control) [33]. 


\section{Bibliography}

[1] Doherty, W. H., "A New High Efficiency Power Amplifier for Modulated Waves," Proc. IRE, Vol. 24, No. 9, September 1936, pp. 1163-1182.

[2] Chireix, H., "High Power Outphasing Modulation," Proc. IRE, Vol. 23, No. 11, November 1935, pp. 1370-1392.

[3] S. Cripps, RF Power Amplifiers for Wireless Communications, 2nd edition, Artech House, 2006.

[4] B. Razavi, Design of Analog CMOS Integrated Circuits. McGraw-Hill; 2001.

[5] F. H. Raab et al., "Power amplifiers and transmitters for RF and microwave," in IEEE Transactions on Microwave Theory and Techniques, vol. 50, no. 3, pp. 814-826, March 2002.

[6] H. Wang, C. Sideris and A. Hajimiri, "A 5.2-to-13GHz class-AB CMOS power amplifier with a $25.2 \mathrm{dBm}$ peak output power at 21.6\% PAE," 2010 IEEE International Solid-State Circuits Conference - (ISSCC), San Francisco, CA, 2010, pp. $44-45$.

[7] W. Ye, K. Ma and K. S. Yeo, "2.5 A 2-to-6GHz Class-AB power amplifier with 28.4\% PAE in 65nm CMOS supporting 256QAM," 2015 IEEE International Solid-State Circuits Conference - (ISSCC) Digest of Technical Papers, San Francisco, CA, 2015, pp. 1-3.

[8] K. Onizuka, S. Saigusa and S. Otaka, "A 1.8GHz linear CMOS power amplifier with supply-path switching scheme for WCDMA/LTE applications," 2013 IEEE International Solid-State Circuits Conference Digest of Technical Papers, San Francisco, CA, 2013, pp. 90-91.

[9] S. Hu, S. Kousai and H. Wang, "A Compact Broadband Mixed-Signal Power Amplifier in Bulk CMOS With Hybrid Class-G and Dynamic Load Trajectory Manipulation," in IEEE Journal of Solid-State Circuits, vol. 52, no. 6, pp. 14631478, June 2017. 
[10] C. M. Andersson et al., "Theory and Design of Class-J Power Amplifiers With Dynamic Load Modulation," in IEEE Transactions on Microwave Theory and Techniques, vol. 60, no. 12, pp. 3778-3786, Dec. 2012.

[11] F. H. Raab, "Class-E, class-C, and class-F power amplifiers based upon a finite number of harmonics," IEEE Trans. Microwave Theory Tech., vol. 47, pp. 1462-1468, Aug. 2001

[12] E. Cope et al., " A 220W 0.0013\% THD+N Class-D audio amplifier with consistent performance up to maximum power level," 2018 IEEE International Solid State Circuits Conference - (ISSCC), San Francisco, CA, 2018, pp. 56-58.

[13] E. Cijvat and H. Sjoland, "Two 130nm CMOS class-D RF power amplifiers suitable for polar transmitter architectures," 2008 9th International Conference on Solid-State and Integrated-Circuit Technology, Beijing, 2008, pp. 1380-1383.

[14] S. Yoo, J. S. Walling, E. C. Woo, B. Jann and D. J. Allstot, " A SwitchedCapacitor RF Power Amplifier," in IEEE Journal of Solid-State Circuits, vol. 46, no. 12, pp. 2977-2987, Dec. 2011.

[15] S. Yoo et al., "A Class-G Switched-Capacitor RF Power Amplifier," in IEEE Journal of Solid-State Circuits, vol. 48, no. 5, pp. 1212-1224, May 2013.

[16] M. Acar (2011). Power amplifiers in CMOS technology : a contribution to power amplifier theory and techniques, Doctoral dissertatio, University of Twente, Enschede, The Netherlands

[17] V. Vorapipat, C. S. Levy and P. M. AsbeckIEEE, "A Class-G Voltage-Mode Doherty Power Amplifier," in IEEE Journal of Solid-State Circuits, vol. 52, no. 12, pp. 3348-3360, Dec. 2017. doi: 10.1109/JSSC.2017.2748283

[18] Kahn, L. R., "Single Sideband Transmission by Envelope Elimination and Restoration," Proc. IRE, Vol. 40, July 1952, pp. 803-806.

[19] M. Hashemi, L. Zhou, Y. Shen, M. Mehrpoo and L. de Vreede, "Highly efficient and linear class-E CMOS digital power amplifier using a compensated Marchand balun and circuit-level linearization achieving $67 \%$ peak DE and -40dbc ACLR without DPD," 2017 IEEE MTT-S International Microwave Symposium (IMS), Honololu, HI, 2017, pp. 2025-2028.

[20] D. C. Cox, "Linear amplification with nonlinear components," IEEE Trans. Commun., vol. COM-23, pp. 1942-1945, Dec. 1974.

[21] Stengel, R., and W. Eisenstadt, "High Efficiency LINC Power Amplifier," Proc. Wireless and Microwave Technology Conf., Chantilly, VA, 1997. 
[22] Chireix, H., "High Power Outphasing Modulation," Proc. IRE, Vol. 23, No. 11, November 1935, pp. 1370-1392.

[23] P. A. Godoy, D. J. Perreault and J. L. Dawson, "Outphasing Energy Recovery Amplifier With Resistance Compression for Improved Efficiency," in IEEE Transactions on Microwave Theory and Techniques, vol. 57, no. 12, pp. 2895-2906, Dec. 2009. doi: 10.1109/TMTT.2009.2033976

[24] D. M. Pozar, Microwave Engineering. 4th edition, Wiley; 2001.

[25] M. P. van der Heijden, M. Acar, J. S. Vromans and D. A. Calvillo-Cortes, "A $19 \mathrm{~W}$ high-efficiency wide-band CMOS-GaN class-E Chireix RF outphasing power amplifier," 2011 IEEE MTT-S International Microwave Symposium, Baltimore, MD, 2011, pp. 1-4.

[26] D. J. Perreault, "A New Power Combining and Outphasing Modulation System for High-Efficiency Power Amplification," in IEEE Trans. on Circuits and Systems I: Regular Papers, vol. 58, no. 11, Aug. 2011.

[27] P. A. Godoy, S. Chung, T. W. Barton, D. J. Perreault and J. L. Dawson, "A 2.4-GHz, 27-dBm Asymmetric Multilevel Outphasing Power Amplifier in 65-nm CMOS," in IEEE Journal of Solid-State Circuits, vol. 47, no. 10, pp. 2372-2384, Oct. 2012.

[28] A. R. Qureshi, M. Acar, J. Qureshi, R. Wesson and L. C. N. de Vreede, "A 112W GaN dual input Doherty-Outphasing Power Amplifier," 2016 IEEE MTTS International Microwave Symposium (IMS), San Francisco, CA, 2016, pp. 1-4. doi: 10.1109/MWSYM.2016.7540194

[29] S. Moloudi and A. Abidi, The Outphasing RF Power Amplifier: A Comprehensive Analysis and a Class-B CMOS Realization, in IEEE Journal of Solid-State Circuits, vol. 48, no. 6, pp. 13571369, June 2013.

[30] L. Ding, J. Hur, A. Banerjee, R. Hezar and B. Haroun, "A 25 dBm Outphasing Power Amplifier With Cross-Bridge Combiners," in IEEE Journal of Solid-State Circuits, vol. 50, no. 5, pp. 1107-1116, May 2015

[31] Z. Hu, L. C. N. de Vreede, M. S. Alavi, D. A. Calvillo-Cortes, R. B. Staszewski and S. He, "A $5.9 \mathrm{GHz}$ RFDAC-based outphasing power amplifier in 40-nm CMOS with $49.2 \%$ efficiency and $22.2 \mathrm{dBm}$ power," in IEEE Radio Frequency Integrated Circuits Symposium (RFIC), 2016, pp. 206-209.

[32] S. M. Bowers, K. Sengupta, K. Dasgupta, B. D. Parker and A. Hajimiri, "Integrated Self-Healing for mm-Wave Power Amplifiers," in IEEE Transactions on 
Microwave Theory and Techniques, vol. 61, no. 3, pp. 1301-1315, March 2013. doi: 10.1109/TMTT.2013.2243750

[33] L. C. N. de Vreede et al., "Outphasing transmitters, enabling digitallike amplifier operation with high efficiency and spectral purity," in IEEE Communications Magazine, vol. 53, no. 4, pp. 216-225, April 2015. doi: 10.1109/MCOM.2015.7081097

[34] R. Zhang, M. Acar, M. P. van der Heijden, M. Apostolidou and D. M. W. Leenaerts, "Generalized Semi-Analytical Design Methodology of Class-E Outphasing Power Amplifier," in IEEE Transactions on Circuits and Systems I: Regular Papers, vol. 61, no. 10, pp. 2951-2960, Oct. 2014. 


\section{Chapter 3}

\section{Load-pulling and PA Break-down}

In chapter 2 , it was shown that for maximum output power and maximum efficiency at this power level, an optimum PA load condition exists. Therefore, load-mismatch can reduce the PA performance. Furthermore, high(er than nominal) transistor voltage and current might occur compromising PAs' reliability.

In this chapter the load-pull concept for the PAs is reviewed assuming linear class A PA operation. Also break-down mechanisms of transistors under high voltage and current conditions are reviewed.

\subsection{Linear PAs and load-pulling}

This section presents a short review of load-effects and load pull analyses, using linear $\mathrm{RF}$ power amplifiers for introduction into the subject and to serve as reference base for the load pull analysis of class-E PA in chapter 4. This section further discusses renormalized load pull contours that are used in the remainder of this thesis.

A very much simplified schematic of a linear RF power amplifier is shown in Fig. 3.1(a). This PA consists of a transistor operating in class A, a matching network and an antenna. For simplicity reasons, this thesis assumes passive lossless matching networks, while impedances are frequently represented in terms of reflection coefficient $\Gamma_{Z}=\left(Z-Z_{0}\right) /\left(Z+Z_{0}\right)$, where $Z_{0}$ is a reference impedance.

It is assumed that the drain (collector) of the transistor is biased to $V_{D D}$ through an RF choke. For a suitable input power level, maximum power is delivered to the load for the condition in which the drain (collector) current range is $\left[0,2 I_{B}\right]$ and the drain (collector) voltage range is $\left[0,2 V_{D D}\right]$. The load impedance that satisfies these 


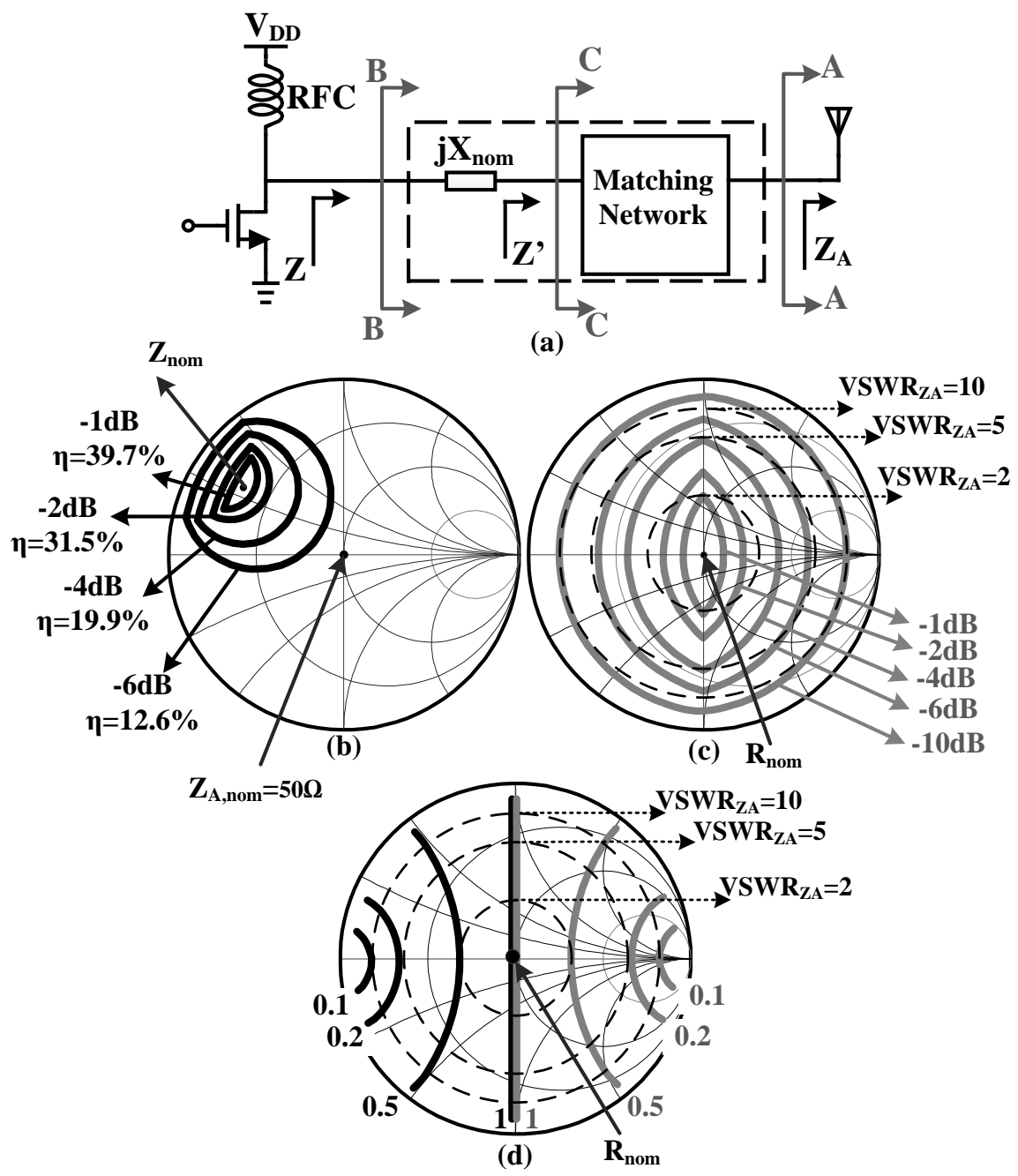

Figure 3.1: (a) Ideal class-A PA with lossless matching network. (b) Power and efficiency contours at the transistor-output (cross section BB). (c) Renormalized power and efficiency contours at the matching network's internal node (cross section CC). (d) Maximum voltage (black) and maximum current (grey) contours of the transistor normalized to those at nominal condition at cross section CC. 
latter two conditions is denoted as $Z_{n o m}=R_{n o m}+j X_{n o m}{ }^{1}$. This load impedance $Z_{\text {nom }}$ is usually matched to a nominal antenna impedance $Z_{A, n o m}$ by the matching network. $Z_{\text {nom }}$ and $Z_{A, \text { nom }}$ are shown in the Smith chart in Fig. 3.1(b), assuming the customary $Z_{A, \text { nom }}=50 \Omega$.

Antenna impedances can vary significantly resulting in VSWRs up to 10:1 [5-7], corresponding to $\left|\Gamma_{Z_{A}}\right|$ that can be up to 0.82 . Any change in the antenna impedance $Z_{A}$ also changes the load $Z$ that is seen by the PA. Following the work by e.g. Cripps [1], the output power and efficiency of the PA can be shown to decrease with increasing $\left|\Gamma_{Z_{A}}\right|$. With input power control to ensure that the PA keeps operating as a linear(ish) PA, equi-output power contours at cross section BB are shown in Fig. 3.1(b). These types of plots are usually referred to as load pull plots. For the plots in Fig. 3.1(b), a $Z_{0}=50 \Omega$ reference impedance was used.

To simplify the analyses in the remainder of this thesis, we mainly use reflection coefficients and Smith charts that are normalized to the (real part of the) nominal load impedance. For that we introduce an impedance $Z^{\prime}$ at cross sections CC in Fig. $3.1(\mathrm{a})$ :

$$
\begin{aligned}
Z^{\prime} & =Z-j X_{n o m} \\
\Gamma_{Z^{\prime}} & =\frac{Z^{\prime}-R_{n o m}}{Z^{\prime}+R_{\text {nom }}}
\end{aligned}
$$

For lossless reciprocal matching networks it can be shown, e.g. [2], that

$$
\left|\Gamma_{Z^{\prime}}\right|=\left|\Gamma_{Z_{A}}\right|
$$

Note that this relation implies that - after renormalization of the reference impedance from $Z_{A, n o m}$ at the antenna plane to $R_{n o m}$ at cross section CC in Fig. 3.1(a) - each constant- $\left|\Gamma_{Z_{A}}\right|$ circle maps onto a constant- $\left|\Gamma_{Z^{\prime}}\right|$ circle with the same radius on a Smith-chart.

Fig. 3.1(c) shows the same power and efficiency contours as Fig. 3.1(b), now at the $Z^{\prime}$ (CC) cross section and normalized to $R_{\text {nom }}$. Note that due to this normalization the load pull contours are now centered around the origin of the Smith chart. Fig. 3.1(c) also shows constant- $\left|\Gamma_{Z_{A}}\right|$ circles for antenna VSWRs of 2, 5 and 10.

In class-A PAs the main effect of load impedance mismatch is the associated decrease in output power and power efficiency. Fig. 3.1(d) shows the voltage magnitude and current magnitude of the transistor for different load impedances, normalized to their respective nominal values for maximum output power. On the left hand side of the Smith chart (current limited operation) the voltage swing is lower than nominal

\footnotetext{
${ }^{1}$ In the previous chapter the optimum impedance was denoted as $R_{o p t}$. Ideally, the optimum impedance for maximum output power and efficiency of class A PAs is purely ohmic. However, in practice, an imaginary part may be required to null e.g. the parasitic drain capacitance.
} 
if the PA includes input power control to ensure class-A operation. Similarly, on the right hand side of the Smith chart in Fig. 3.1(d) the PA's output voltage is limited and the output current is lower than nominal with proper input power control. Hence, using input power control to ensure linear operation, neither the PA's output voltage nor its output current gets higher than its value under nominal conditions and reliability is not compromised by load impedance variations. For non-linear amplifiers the situation is very much different, as will be shown in chapter 4 .

\subsection{Transistor breakdown mechanisms}

Several mechanisms can degrade CMOS-based PA performance. In this section we briefly discuss the well-known degradation mechanisms: gate oxide breakdown, hot carrier degradation, punch through, drain-bulk breakdown and electro-migration. For more comprehensive details, we refer readers to $[3,4]$.

\subsubsection{Gate oxide breakdown}

When the gate-source and the gate-drain voltages $\left(V_{G S}\right.$ and $\left.V_{G D}\right)$ exceed pre-specified values, determined by the technology, a conductive path in the gate-oxide of a MOS device is formed. These pre-specified breakdown voltages depend on the oxide thickness and decreases with new CMOS process generation. Gate oxide breakdown can result in permanent damage to the gate oxide causing device performance degradation $[8-11]$.

\subsubsection{Hot carrier degradation}

When high drain-source voltages are applied to short channel devices, the lateral fields can be extremely high. This can result in carriers with a very high energy, denoted as "hot energy carriers". These hot energy carriers can collide with the lattice before arriving at the drain causing impact ionization, hence surface defects and trapped charges in the gate oxide or oxide/silicon interface. These surface defects and trapped charge can result in reduced carrier mobility in the channel and shifted local threshold voltage hence e.g. increased on-resistance and knee voltage of the device and as a result a gradual performance degradation (unlike gate-oxide breakdown which is intrinsically catastrophic) [12-14].

\subsubsection{Punch through}

In the absence of gate bias, a CMOS device can be modeled as two back to back diodes (drain-bulk and source-bulk diode). In this case a very small current passes through 
the device. However, if the drain-source voltage increases, the drain-bulk depletion region extends to (eventually) meet the source-bulk depletion region (shown in Fig. 3.2 ), thereby diminishing the potential barrier, resulting in a very high drain-source current [3].

This phenomenon can creates hot carriers that, similar to hot carrier degradation, can gradually degrade the CMOS device performance.
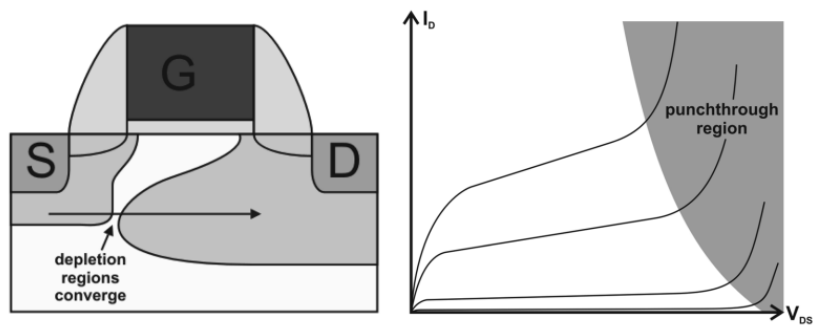

Figure 3.2: (a) Punchthrough in a MOS transistor. (b) The effect on the I-V curve. [3]

\subsubsection{Drain-bulk breakdown}

In a standard bulk CMOS process, there is a parasitic diode associated with drainbulk junction. For normal operation, the bulk is connected to ground while the drain connection undergoes positive voltage excursions. The break-down voltage of this parasitic diode is specified by the technology and the drain voltage should always be kept below this voltage [3].

\subsubsection{Electromigration}

Electromigration is the displacement of conductor atoms due to high conductor current density. Under load-mismatch conditions and for switch-mode class-E PAs, the current density in the metal routings can increase significantly, possibly leading to electromigration. Electromigration, similar to hot carrier degradation, gradually degrades the performance due to e.g. increased loss associated with the increase in resistance of the metal routings $[15,16]$.

The break-down mechanisms reviewed in this chapter limit the voltage excursions across and current excursions through the terminals of a CMOS transistor. Having a relation between a class-E PA loading and these excursions, safe operating areas on the Smith chart for which the excursions are below the limits can be identified. Current limited break-downs can be mitigated by design, e.g. larger CMOS size, wider 
connections, more contacts etc. However, voltage limited break-downs can have severe permanent performance degradation and must be avoided or limited. In chapter 4, class-E PAs safe operating area is discussed and guildlines are given to extend it. Detailed discussion on the practical implementation is given in chapter 7 . 


\section{Bibliography}

[1] S. Cripps, RF Power Amplifiers for Wireless Communications, 2nd edition, Artech House, 2006.

[2] K. Kurokawa, "Power Waves and the Scattering Matrix", Microwave Theory and Techniques, IEEE Transactions on, vol. 13, no. 2, pp. 194-202, March 1965, DOI: 10.1109/TMTT.1965.1125964

[3] I. Aoki, S. Kee, R. Magoon, R. Aparicio, F. Bohn, J. Zachan, G. Hatcher, D. McClymont and A. Hajimiri, "A Fully-Integrated Quad-Band GSM/GPRS CMOS Power Amplifier," Solid-State Circuits, IEEE Journal of, vol.43, no.12, pp.2747,2758, Dec. 2008 doi: 10.1109/JSSC.2008.2004870

[4] B. Razavi, Design of Analog CMOS Integrated Circuits. McGraw-Hill; 2001.

[5] E.L. Firrao, A.-J. Annema and B. Nauta, "An Automatic Antenna Tuning System Using Only RF Signal Amplitudes", IEEE TCASII, pp. 833-837 vol. 55, no. 9, September 2008.

[6] K. Boyle and M. Leitner, "Mobile phone antenna impedance variations with real users and phantoms", in proc. iWAT, pp. 420-423, Hong Kong, 2011.

[7] K.R. Boyle, Y. Yuan, and L.P. Ligthart, "Analysis of Mobile Phone Antenna Impedance Variations With User Proximity", IEEE Transactions on Antennas and Propagation, vol. 55, no. 2, pp. 364372, Feb 2007.

[8] H. Yang, J.S. Yuan, Y. Liu and E. Xiao "Effect of gateoxide breakdown on RF performance", in IEEE Transactions on Device and Materials Reliability, vol. 3 issue 3, pp.93-97, September 2003.

[9] G.T. Sasse, F.G. Kuper and J. Schmitz, "MOSFET Degradation Under RF Stress", in IEEE Transactions on Electron Devices, vol. 55, issue 11, November 2008 .

[10] C. Hu, "Gate Oxide Scaling Limits and Projection", IEDM December 1996. 
[11] F. Monsieur, E. Vincent, D. Roy, S. Bruyere, J.C. Vildeuil, G. Pananakakis and G. Ghibaudo, "A thorough investigation of progressive breakdown in ultrathin oxides. Physical understanding and application for industrial reliability assessment", in Proc. IRPS 2002 pp. 45-54.

[12] G.T. Sasse and J. Bisschop, "The hot carrier degradation rate under AC stress", 2010 IEEE International Reliability Physics Symposium, pp. 830-834, May 2010.

[13] J.S. Yuan, H. Tang, "CMOS RF Design for Reliability Using Adaptive GateSource Biasing", in IEEE Transactions on Electron Devices, vol. 55, issue 9, September 2008.

[14] P. Sharma et al., "Modeling of Hot-Carrier Degradation in nLDMOS Devices: Different Approaches to the Solution of the Boltzmann Transport Equation", in IEEE Transactions on Electron Devices, vol. 62, no. 6, pp. 1811- 1818, June 2015.

[15] C. Kim, "Electromigration in Thin Films and Electronic Devices: Materials and Reliability". Oxford: Woodhead Pub.

[16] C. Constantinescu, "Trends and challenges in VLSI circuit reliability", IEEE Micro, vol. 23, no. 4, 2003. 


\section{Chapter 4}

\section{Load-mismatch Sensitivity of Class-E Power Amplifiers}

\subsection{Introduction}

Switched-mode power amplifiers (SMPAs) ${ }^{1}$ have shown great potential for power efficient amplification of RF signals in the past years [2]. Most types of SMPAs incorporate a number of resonant tanks both to ensure e.g. zero-voltage switching (ZVS) and zero-slope switching (ZSS) conditions and to ensure waveform shaping of the output signal $[2]-[5]$. SMPAs are usually designed for nominal operating conditions where they hence reach optimum performance.

For non-nominal load conditions, the resonant tanks in the SMPAs may get detuned which leads to degraded performance in terms of output power and power efficiency. Closely related to this, detuned tanks result in non-nominal voltage excursions across the switch and non-nominal currents through the switch that may lead to increased degradation of the switch. For RF power amplifiers, typically loadmismatch due to a changing antenna environments is the major external effect causing non-nominal operating conditions [6-8].

The sensitivity of the class-E PAs to circuit components variations was studied in $[9,10]$. In $[9,10]$, from a load-mismatch point of view, the effect of the loadmismatch was investigated for very specific cases of having mismatch (only) on the real part or (only) on the imaginary part of the PA load, separately. However, under load-mismatch and for high voltage standing wave ratios (VSWRs) both the real and the imaginary parts of the PA load (simultaneously) can heavily change. The study

\footnotetext{
${ }^{1}$ This chapter consists of material previously published in IEEE Transactions on Microwave Theory and Techniques (T-MTT) [1] which was re-structured for readability in thesis form.
} 
provided by [9] was also employed by $[11,12]$ to mathematically analyze outphasing class-E PAs. However, the derivations are for specific cases of class-E PAs, assuming an infinite and ideal dc-feed inductor, an ideal lossless switch and 50\% duty cycle while no reliability related issues were addressed.

Some of the well-known non-idealities on the actual implementation of the classE SMPAs were studied previously. The effect of the switch conduction loss on the performance of the class-E PAs can be found in e.g. [13-15]. The work in [13] aims at designing class-E PAs with sufficiently low maximum voltages and sufficiently high efficiencies with optimizing the switching conditions while $[14,15]$ study the effect of the switch conduction loss for specific switching conditions (ZVS and ZSS in [14] and ZSS only in [15]). The limited negative voltage excursions across the switch was briefly discussed in [9] for the specific cases of class-E PAs. The work in $[16,17]$ utilize a parallel diode to (intentionally) shunt negative voltage excursions across the switch when changing the real part of the load from its nominal value to zero. However, the work in $[16,17]$ holds for a limited range of the loads (only real loads in the range of zero to the nominal value) for a class-E amplifier designed with infinite dc-feed inductor operating at low frequencies (below several $\mathrm{MHz}$ ). The effects of the limited $Q_{\text {loaded }}$ on the design equations of a class-E PA can be found in [18], however, again, for a specific switching conditions (ZVS and ZSS).

In [19], we used the load-pull contours of the ideal class-E PAs to (mathematically) generalize the theory of the outphasing class-E PAs and to improve the back-off efficiency of such PAs. There, only the results of ideal load-pull analyses for a (very) limited area of the class-E PA loads (on the Smith chart) were discussed without providing the derivations of the contours. This chapter presents mathematical analyses of the effect of load-mismatch on the behavior, performance and reliability of class-E SMPAs, complemented by simulation and measurement results. In the mathematical analyses in this chapter, the class-E amplifiers are kept as general as possible, hence with arbitrary finite dc-feed inductor, arbitrary duty cycle and arbitrary switching conditions (ZVS and ZSS are not required to be satisfied for the nominal load) and also including the most common non-idealities in actual class-E PA implementations. The non-idealities include the loss of the dc-feed inductor (represented by the quality factor of the inductor), the conduction loss of the switch, the limited loaded quality factor of the output series filter $\left(Q_{\text {loaded }}\right)$, limited negative voltage excursions across the switch and the dependency of the switch conduction loss on the load-mismatch.

The objectives of this chapter include, firstly, developing a basic yet general theoretical model to study the load-mismatch sensitivity of class-E PAs and, secondly, providing the theory behind the load-pull contours employed in [19] and in the remainder of this thesis. The model is not limited to any specific frequency, output power level, technology nor to any specific class-E design parameters. This basic model provides a(n initial) picture of the load-mismatch effects by providing load-pull-like 
representation of various performance and reliability related metrics of a class-E PA. We (re)normalize voltages, power levels, currents and use Smith chart representations to be as independent from frequency, power and technology as possible. The presented general model is followed by a case study utilizing the model to study load-mismatch effects on a class-E PA. For the case study, practical class-E design parameters are assumed and from there the impact of changing the so-called relative resonance frequency $q$ and the duty cycle scaling factor $d[20]$, are discussed. Non-idealities further impact the class-E behavior. In contrast to $[9,13-15,18]$, the impact of the most dominant non-idealities are analyzed for load-mismatch with VSWRs up to 20 using theoretical derivations and simulations and for VSWRs up to 10 in measurements. Finally, load-pull measurement results on a class-E PA implemented in $65 \mathrm{~nm}$ CMOS technology confirm our model and analyses of the impact of the non-idealities.

The switch in class-E PAs is usually implemented with one or multiple transistors. The maximum voltage that the transistor can handle (determined by the technology) puts a limit on supply voltage $V_{D D}$ [13]. The size of the transistor and the metal routings are designed by finding the best compromise between the area (parasitics), power efficiency and electromigration rules, see e.g. [21,22]. To achieve high output power, power efficiency and life-time, $V_{D D}$ and the transistors are typically designed to marginally handle the maximum switch voltage and the maximum switch current. In that case, higher-than-nominal values for the switch voltage (leading to e.g. oxide breakdown in CMOS technology) and for the switch current (leading to e.g. electromigration) cause reliability issues [23-25]. These reliability issues can be alleviated by inducing design margins at the cost of e.g. lower output power and a lower power efficiency. The load-pull-like representation of the reliability related metrics of a classE PA in this chapter allows to optimize design margins to handle specific mismatch conditions and as a result allows to define a safe operating area for the class-E PA on the Smith chart.

There are numerous papers on the derivation of the design equations of the class-E PAs and the changing of the design parameters on the performance and the reliability of the class-E PAs. Design equations for the arbitrary finite dc-feed inductor, arbitrary duty cycle and ZVS and ZSS conditions can be found in e.g. [20]. [15,26-29] derive similar equations but for a more relaxed switching conditions; only ZVS or ZSS is satisfied. The effect of changing the design parameters $d$ and $q$ on the design equations was discussed in [30] for ZVS and ZSS conditions and [21] studied the effect of changing $d$ for ZVS condition. Under load-mismatch conditions the ZVS and ZSS conditions, both are violated. Therefore, to be able to mathematically study load-mismatch effects, new mathematical design equations for general/arbitrary switching conditions were derived similar to [13] but now also including the loss of the dc-feed inductor as well as the switch conduction loss. The outline of this chapter is described below.

Section 4.2 presents a brief recap on the basics of class-E SMPAs. The mathe- 
matical foundation underlying the results in section 4.2 is extended in section 4.3 , to be able to mathematically derive the effects of load-mismatch on the performance of class-E SMPAs. Section 4.4 presents the analyses of the effects of load-mismatch, presented in load-pull plots, on output power, power efficiency and on the switch voltage and switch current. For the theoretical part in this section, infinite loaded quality factor for the series tank ( $Q_{\text {loaded }}$ ) is assumed [20]. This section includes simulation results and discussions of the effect of a finite $Q_{\text {loaded }}$ for the series tank, assuming $Q_{\text {loaded }}=5$ under nominal load condition.

Section 4.5 combines the load-pull effects on maximum switch voltage and current from section 4.4 to determine safe operation region for class-E PAs. The impact of a few non-idealities that occur in actual SMPA circuit implementations is discussed in section 4.6. Finally, load-pull measurement results of a class-E PA implemented in $65 \mathrm{~nm}$ CMOS technology at $1.4 \mathrm{GHz}$ are given in section 4.7. The overall conclusions are summarized in section 4.8 .

\subsection{Class-E Power Amplifier Basics}

Class-E PAs are switched-mode RF amplifiers, that in a simplified form consist of a switch and two $L C$-tanks. In Fig. 4.1(a), the MOS transistor with its square wave input signal implements the switch, while $L$ and $C$ form the primary resonance tank and where $L_{0}$ and $C_{0}$ form the band pass filter (second tank) tuned to the transmit band.

The switch transistor is driven by (ideally) a square wave signal defined as [20]

$$
S \triangleq\left\{\begin{array}{lr}
\text { on; } & 0<\omega_{0} t<d \pi \\
\text { off; } & d \pi<\omega_{0} t<2 \pi
\end{array}\right.
$$

where $d$ is a real number in the interval $[0,2]$ and $d=1$ corresponds to $50 \%$ duty cycle and where $\omega_{0}$ is the driving square wave angular frequency. For conventional ZVS and ZSS class-E PAs [31,32], parameters $\alpha$ respectively $\beta$ in Fig. 4.1(b) are zero. In general ZVS or ZSS conditions do not need to be satisfied leading to non-zero $\alpha$ and $\beta$, which are defined as [15], [26]:

$$
\begin{aligned}
& v_{C}\left(\frac{2 \pi}{\omega_{0}}\right)=\alpha V_{D D} \\
& \frac{d v_{C}}{d t}\left(\frac{2 \pi}{\omega_{0}}\right)=\beta \omega_{0} V_{D D}
\end{aligned}
$$

where $v_{C}$ is the drain (switch) voltage and $V_{D D}$ is the supply voltage ${ }^{2}$.

\footnotetext{
${ }^{2}$ In this chapter the analysis is conducted at a constant supply voltage.
} 


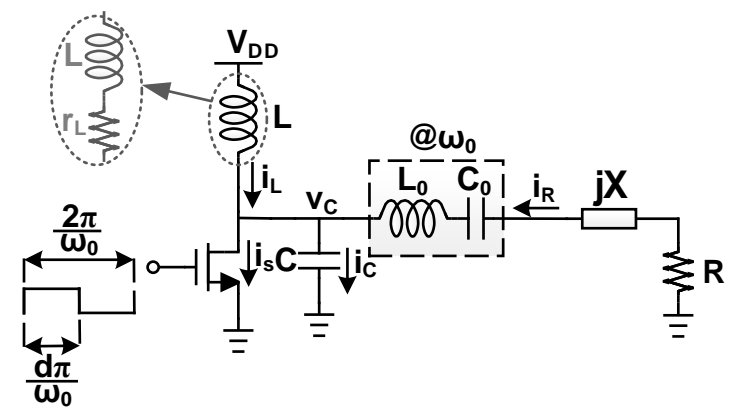

(a)

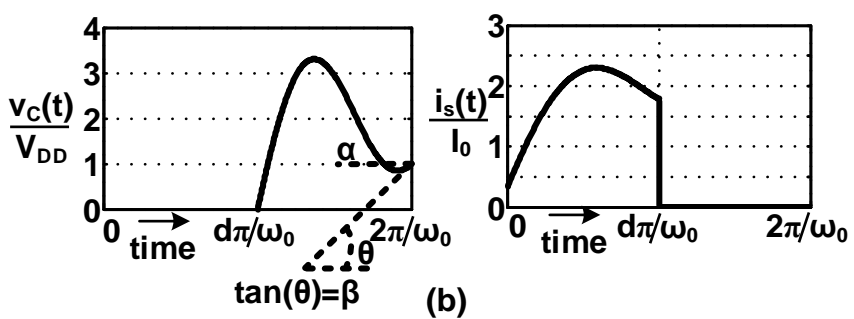

Figure 4.1: (a) Single Ended Class-E PA. (b) Normalized switch (transistor) voltage to $V_{D D}$ and normalized switch current to the average supply current $I_{0}$ for a class-E PA using a lossless switch.

For ideal class-E operation, the load impedance $R+j X$, the (angular) signal frequency $\omega_{0}$, supply voltage $V_{D D}$ and output power level $P_{\text {out }}$ and a number of properties of the Class-E PA itself must be well chosen. PA properties to be well chosen include duty cycle $d$, switch on-resistance $R_{o n}$ and component values.

For general switching conditions, the relations between all class-E PA parameters are formulated in e.g. [13] in so-called the K-design set, see Table 4.1. In [13], all $K$-values in the K-design set are non-linear functions of parameters $q, d, m, \alpha$ and $\beta$. The parameters $q, d$ and $m$ are respectively the relative resonance frequency of the $L C$-tank, the duty cycle scaling factor as defined in Fig. 4.1 and the relative switch on-resistance:

$$
\begin{aligned}
q & =\frac{1}{\omega_{0} \sqrt{L C}} \\
m & =\omega_{0} R_{o n} C
\end{aligned}
$$

The first part of this chapter mathematically describes the effects of load impedance mismatch on the behaviour of class-E PAs, in terms of reliability, output power and efficiency. For this, a nominal class-E PA is subjected to non-nominal load impedance conditions. To do this in a mathematical way, the parameters $q, d, m, \alpha, \beta$ and also the quality factor of the dc-feed inductor $L\left(Q_{L}\right)$, are taken as the inputs to the K- 
Table 4.1: K-design set.

$$
K_{L}=\frac{L \omega_{0}}{R} \quad K_{C}=\omega_{0} R C \quad K_{X}=\frac{X}{R} \quad K_{P}=\frac{R P_{\text {out }}}{V_{D D}^{2}}
$$

design set and a full $K=K\left(q, d, m, Q_{L}, \alpha, \beta\right)$ design set was derived in a similar way as in $[13-15,20,21,26]$ but now geared towards load-pull style analyses. To include the parameter $Q_{L}$ in the analysis, we assume a resistance $r_{L}$ in series with inductor $L$, shown in Fig. $4.1(\mathrm{a})$, where $Q_{L}=\frac{L \omega_{0}}{r_{L}}$. For an ideal dc-feed inductor $L$ we have $r_{L} \rightarrow 0$ and hence $Q_{L} \rightarrow \infty$. The full derivation of the K-design set is not the topic of this chapter and only a short review in the context of load-mismatch is given in section 4.3 .

\subsection{Non-ZVS and non-ZSS Class-E PA}

To satisfy a pre-determined switching conditions of a class-E PA (e.g. ZVS and ZSS), a specific (nominal) load should be presented to the PA $[15,20,26]$. As a result of a non-nominal load, the switching conditions change. In this section we consider class-E PAs with an arbitrary switching condition.

The switching conditions of the non-ZVS and non-ZSS class-E PA are shown in Fig. 4.1 and are mathematically given in (4.2) and (4.3). For simplicity, a few assumptions are made similar to the approach in e.g. [14]. Firstly, the switch onresistance $R_{\text {on }}$ is assumed to be constant while the off-state resistance is assumed to be infinite. Note that real power losses are only due to non-zero $R_{o n}$, non-zero $r_{L}$ and discharging capacitor $\mathrm{C}$ at the switching moment. Secondly, a high loaded quality factor for the series filter $L_{0}-C_{0}$, i.e. $Q_{\text {loaded }}=\frac{L_{0} \omega_{0}}{R} \rightarrow \infty$, is assumed in the theoretical analyses, for which the load current, $i_{R}$ in Fig. 4.1, can be assumed to be sinusoidal, i.e. $i_{R}=I_{R} \sin \left(\omega_{0} t+\varphi\right)$.

A full mathematical derivation of the switch voltage $v_{C}(t)$ for $\omega_{0} t \in[d \pi, 2 \pi]$ (denoted as $\left.v_{C \text {,off }}(t)\right)$ and for $\omega_{0} t \in[0, d \pi]$ (denoted as $\left.v_{C \text {,on }}(t)\right)$ can be readily derived by performing KCL at the switching node $v_{C}$ and using the continuity of the capacitor voltage $v_{C}$ and the inductor current $i_{L}$ at the switching moments. The switch current for the time period that the switch is closed, $\omega_{0} t \in[0, d \pi]$, can then be written as $i_{s}(t)=\frac{v_{C, \text { on }}(t)}{R_{o n}}$. With the definition of $p$ as

$$
p=\frac{\omega_{0} L I_{R}}{V_{D D}},
$$

it can be shown that

$$
\frac{v_{C, \mathrm{on}}}{V_{D D}}=c_{1} e^{s_{1} \omega_{0} t}+c_{2} e^{s_{2} \omega_{0} t}+\frac{m}{m+\frac{1}{q^{2} Q_{L}}}+
$$




$$
\begin{gathered}
A p \sin \left(\omega_{0} t+\varphi\right)+B p \cos \left(\omega_{0} t+\varphi\right) \\
\frac{v_{C, \text { off }}(t)}{V_{D D}}=c_{1}^{\prime} e^{s_{r} \omega_{0} t} \cos \left(s_{i} \omega_{0} t\right)+c_{2}^{\prime} e^{s_{r} \omega_{0} t} \sin \left(s_{i} \omega_{0} t\right)+ \\
1+A^{\prime} p \sin \left(\omega_{0} t+\varphi\right)+B^{\prime} p \cos \left(\omega_{0} t+\varphi\right)
\end{gathered}
$$

where $s_{r}=\frac{-1}{2 Q_{L}}, s_{i}=q \sqrt{1-\frac{1}{4 Q_{L}^{2} q^{2}}}$ and where, $s_{1}, s_{2}, A, B, A^{\prime}$ and $B^{\prime}$ can be written as a function of $q, m$ and $Q_{L}$ while $c_{1,2}, c_{1,2}^{\prime}, p$ and initial phase $\varphi$ depend on all K-design set parameters $q, d, m, Q_{L}, \alpha$ and $\beta$ (not shown here for length reasons).

\subsection{1 $K$-design set summary}

The power delivered by the supply voltage is ideally fully dissipated in the load. However, a non-zero switch on-resistance, limited $Q_{L}$ and non-ZVS conditions give rise to extra power dissipation, making up losses. Denoting the average supply current as $I_{0}$, i.e. $\left\langle i_{L}(t)>=I_{0}\right.$, and denoting the output power and loss power respectively as $P_{\text {out }}$ and $P_{\text {Loss }}$, then in equation,

$$
\begin{aligned}
& V_{D D} I_{0}=P_{\text {out }}+P_{\text {Loss }} \\
& P_{\text {out }}=\frac{1}{2} R I_{R}^{2} \\
& I_{0}=\frac{\omega_{0}}{2 \pi} \int_{0}^{\frac{2 \pi}{\omega_{0}}} i_{L}(t) d t=\frac{\omega_{0}}{2 \pi} \int_{0}^{\frac{d \pi}{\omega_{0}}} i_{s}(t) d t \\
& P_{\text {Loss }}=\frac{\omega_{0}}{2 \pi}\left(R_{\text {on }} \int_{0}^{\frac{d \pi}{\omega_{0}}} i_{s}^{2}(t) d t+r_{L} \int_{0}^{\frac{2 \pi}{\omega_{0}}} i_{L}^{2}(t) d t\right)
\end{aligned}
$$

For an ideal switch with $R_{o n}=0(m=0)$ and an ideal dc-feed inductor with $Q_{L} \rightarrow \infty$, (4.12) reduces to the power loss due to discharging capacitor $C$ :

$$
\left.P_{L o s s}\right|_{m=0, Q_{L} \rightarrow \infty}=\frac{\frac{1}{2} C\left(\alpha V_{D D}\right)^{2}}{\frac{2 \pi}{\omega_{0}}}=\frac{\omega_{0}}{4 \pi} C\left(\alpha V_{D D}\right)^{2}
$$

Using (4.9) and (4.12) along with the definition of $q$ and $K_{L}$ in Table $4.1, K_{L}$ can be obtained as

$$
K_{L}=\frac{\pi m p^{2} q^{2} / \omega_{0}}{\int_{0}^{\frac{d \pi}{\omega_{0}}} \frac{v_{C, o n}(t)}{V_{D D}}-\left(\frac{v_{C, o n}(t)}{V_{D D}}\right)^{2} d t-\int_{0}^{\frac{2 \pi}{\omega_{0}}} r_{L} R_{o n}\left(\frac{i_{L}(t)}{V_{D D}}\right)^{2} d t}
$$

where $v_{C, \text { on }}(t)=R_{\text {on }} \cdot i_{s}(t)$ is the switch voltage during the on-state and $i_{L}$ is the supply current, shown in Fig. 4.1. Equation (4.14) can be explicitly rewritten into $K_{L}\left(q, d, m, Q_{L}, \alpha, \beta\right) . K_{C}$ can then readily be derived from the definition of $q$ as

$$
K_{C}\left(q, d, m, Q_{L}, \alpha, \beta\right)=\frac{1}{q^{2} K_{L}\left(q, d, m, Q_{L}, \alpha, \beta\right)} .
$$


Substituting $I_{R}=\sqrt{\frac{2 P_{\text {out }}}{R}}$ into (4.6) then yields

$$
K_{P}\left(q, d, m, Q_{L}, \alpha, \beta\right)=\frac{p^{2}\left(q, d, m, Q_{L}, \alpha, \beta\right)}{2 K_{L}^{2}\left(q, d, m, Q_{L}, \alpha, \beta\right)}
$$

$K_{X}$ was derived in [14] as

$$
K_{X}=\frac{V_{X}}{V_{R}}
$$

where $V_{X}$ and $V_{R}$ are two quadrature Fourier components of the switch voltage. Following a similar approach as in [14] yields an expression for $K_{X}$ that depends only on K-design set parameters.

\subsubsection{Waveform maxima}

The switch current in the time interval of $0<\omega_{0} t<d \pi$ is obtained from $i_{s}(t)=$ $\frac{v_{C, \text { on }}(t)}{R_{\text {on }}}$ where, $v_{C, \text { on }}$ is given in (4.7). The maximum switch current, $I_{\text {smax }}$, can be written as

$$
I_{\text {smax }}=\frac{V_{D D}}{R_{o n}} f_{I_{1}}\left(d, q, m, Q_{L}, \alpha, \beta\right)
$$

where $f_{I_{1}}$ is a function of $\mathrm{K}$-design set parameters (not shown here for length reasons). Using (4.4), (4.5) and (4.6), (4.18) can be rewritten as to obtain the maximum switch current normalized to $I_{0}$ :

$$
\frac{I_{\text {smax }}}{I_{0}}\left(d, q, m, Q_{L}, \alpha, \beta\right)=\frac{f_{I_{1}}\left(d, q, m, Q_{L}, \alpha, \beta\right)}{m p q^{2} f_{I_{2}}\left(d, q, m, Q_{L}, \alpha, \beta\right)}
$$

where $f_{I_{2}}=\frac{I_{0}}{I_{R}}$ depends only on K-design set parameters and can be derived from (4.11) along with the definition of $q$ and $p$ which is not shown here for length reasons.

An expression for the switch voltage in the time interval of $0<\omega_{0} t<2 \pi$ is given in (4.7) and (4.8). Straightforward calculus yields the location of the maximum and the minimum of the switch voltage. The maximum and the minimum in the normalized switch voltage can then be written as a function of K-design set parameters which are not shown here again for length reasons.

\subsubsection{Efficiency}

The drain efficiency is given by $\eta=1-P_{\text {Loss }} /\left(V_{D D} I_{0}\right)$. Using (4.4), (4.6), (4.12) and $f_{I_{2}}=\frac{I_{0}}{I_{R}}$, this relation can be written as

$$
\eta=1-\frac{\omega_{0} \int_{0}^{\frac{d \pi}{\omega_{0}}}\left(\frac{V_{C_{o n}}(t)}{V_{D D}}\right)^{2}+r_{L} R_{o n}\left(\frac{i_{L}(t)}{V_{D D}}\right)^{2} d t}{2 \pi m p q^{2} f_{I_{2}}\left(d, q, m, Q_{L}, \alpha, \beta\right)}
$$


which only depends on the K-design set parameters. For an ideal switch, with $R_{o n}=$ 0 , and an ideal dc-feed inductor, with $Q_{L} \rightarrow \infty,(4.20)$ can be simplified to

$$
\eta=1-\frac{\alpha^{2}}{8 \pi p q^{2}\left(\frac{\pi d^{2}}{8 p}-\frac{d}{4} \sin (\varphi)+\frac{\cos (\varphi)-\cos (d \pi+\varphi)}{4 \pi}\right)+\alpha+\pi \beta d}
$$

\subsubsection{Non-ZVS and non-ZSS class-E summary}

The previous 3 subsections showed that the performance of class-E PAs is affected by K-design set parameters $d, q, m, Q_{L}, \alpha$ and $\beta$. Parameters $d, q, m$ and $Q_{L}$ are typically fixed by design, as are parameters $\alpha$ and $\beta$ assuming the nominal load impedance. Due to load impedance (antenna) mismatch, parameters $\alpha$ and $\beta$ change which in effect will deterministically change the performance of the class-E PA according to the relations listed in the previous 3 subsections. In depth analyses of these load-pull effects for class-E PAs are described in the next section.

\subsection{Antenna Load-Mismatch Effects On Class-E Per- formance}

\subsubsection{Effects on PA load}

Without loss of generality, we assume a class-E PA that is designed for a certain set of nominal conditions $\left(\alpha=\alpha_{n o m}\right.$ and $\left.\beta=\beta_{\text {nom }}\right)$ with arbitrary $d, q, m$ and $Q_{L}$. For this class-E PA, there is a specific nominal $\mathrm{K}$ design-set with elements $K_{L, \text { nom }}=\frac{L \omega_{0}}{R}$, $K_{C, \text { nom }}=\omega_{0} R C, K_{X, n o m}=\frac{X}{R}$ and $K_{P, n o m}=\frac{R P_{\text {out }, \text { nom }}}{V_{D D}^{2}}$ where $Z_{\text {nom }}=R+j X$ is the nominal load impedance for the nominal output power $P_{\text {out,nom }}$ with nominal efficiency $\eta_{\text {nom }}$.

This nominal load impedance $Z_{\text {nom }}$ is typically transformed into a nominal $Z_{A, \text { nom }}=$ $50 \Omega$ antenna impedance using a matching network, see e.g. Fig. 4.2. For simplicity reasons, in this chapter a lossless reciprocal matching network is assumed. The reflection coefficient at the antenna feed point due to changing antenna impedance is then $\Gamma_{Z_{A}}=\frac{Z_{A}-Z_{A, n o m}}{Z_{A}+Z_{A, n o m}}$. Any mismatch at the antenna (cross section AA in Fig. 4.2) will change the PA load $Z$. For normalization purposes, we rewrite $Z$ as

$$
Z=k R+j\left(X+k^{\prime} R\right)
$$

where $k>0$ and $k^{\prime}$ are two real numbers and for the nominal load condition $k=1$ and $k^{\prime}=0\left(Z=Z_{\text {nom }}=R+j X\right)$. We introduce an impedance $Z^{\prime}$ at cross sections 


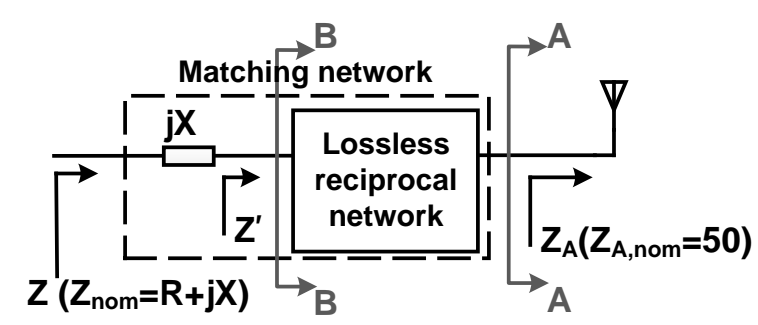

Figure 4.2: A lossless reciprocal matching network transforms the antenna impedance $Z_{A}$, represented by its nominal value $Z_{A, n o m}=50 \Omega$ to the PA load $Z$, represented by its nominal value $Z_{\text {nom }}=R+j X$.

BB in Fig. 4.2:

$$
\begin{aligned}
Z^{\prime} & =Z-j X \\
\Gamma_{Z^{\prime}} & =\frac{Z^{\prime}-R}{Z^{\prime}+R}
\end{aligned}
$$

For lossless reciprocal matching networks it can be shown, e.g. [33], that

$$
\left|\Gamma_{Z^{\prime}}\right|=\left|\Gamma_{Z_{A}}\right|
$$

Note that this relation implies that - after renormalization of the reference impedance from $Z_{A, n o m}$ at the antenna plane to $R$ at cross section $\mathrm{BB}$ in Fig. 4.2 - each constant- $\left|\Gamma_{Z_{A}}\right|$ circle maps onto a constant- $\left|\Gamma_{Z^{\prime}}\right|$ circle with the same radius in a Smith-chart.

Using (4.23), (4.24) and (4.25) along with the definition of VSWR at cross section AA in Fig. 4.2 as $\operatorname{VSWR}_{Z_{A}}=\frac{1+\left|\Gamma_{z_{A}}\right|}{1-\left|\Gamma_{Z_{A}}\right|}$,

$$
\operatorname{VSWR}_{Z_{A}}=\frac{\sqrt{(k+1)^{2}+k^{\prime 2}}+\sqrt{(k-1)^{2}+k^{\prime 2}}}{\sqrt{(k+1)^{2}+k^{\prime 2}}-\sqrt{(k-1)^{2}+k^{\prime 2}}}
$$

For any positive real number $a$, the range of $k$ and $k^{\prime}\left(k-k^{\prime}\right.$ surface) and as a result the range of the PA loads $Z$ for which $\mathrm{VSWR}_{Z_{A}} \leq a$ follows straightforwardly from (4.26).

\subsubsection{K-design set}

The load impedance seen by the class-E PA is defined in (4.22). The values of the K-design set elements under mismatch conditions, $K_{L}, K_{C}, K_{X}, K_{P}$, can now be 
calculated as a function of $k$ and $k^{\prime}$ (and hence $\operatorname{VSWR}_{Z A}$ ) as follows

$$
\begin{aligned}
K_{L} & =\frac{L \omega_{0}}{\operatorname{Re}(Z)}=\frac{L \omega_{0}}{k R}=\frac{K_{L_{\text {nom }}}}{k} \\
K_{C} & =\omega_{0} \operatorname{Re}(Z) C=k \omega_{0} R C=k K_{C_{\text {nom }}} \\
K_{X} & =\frac{\operatorname{Im}(Z)}{\operatorname{Re}(Z)}=\frac{X+k^{\prime} R}{k R}=\frac{K_{X_{\text {nom }}}}{k}+\frac{k^{\prime}}{k} \\
K_{P} & =\frac{\operatorname{Re}(Z) P_{\text {out }}}{V_{D D}^{2}}=k \frac{P_{\text {out }}}{P_{\text {out }, \text { nom }}} K_{P_{\text {nom }}}
\end{aligned}
$$

where $P_{\text {out }}$ and $P_{\text {out,nom }}$ are the output power under mismatch and nominal conditions, respectively. The values of $\alpha$ and $\beta$ corresponding to this K-design set under mismatch conditions determine the performance of the PA with load-mismatch. With the above equations, these $\alpha$ and $\beta$ are functions of $k$ and $k^{\prime}$ and can be calculated from e.g. (4.27) and (4.29) by solving

$$
\begin{aligned}
& K_{L}\left(q, d, m, Q_{L}, \alpha, \beta\right)=\frac{K_{L_{n o m}}}{k} \\
& K_{X}\left(q, d, m, Q_{L}, \alpha, \beta\right)=\frac{K_{X_{n o m}}}{k}+\frac{k^{\prime}}{k}
\end{aligned}
$$

where the K-design set equations are summarized in the previous section. Having the values of $\alpha$ and $\beta$, all performance parameters of the PA are known. Hence, for a specific range of antenna load impedances, properties such as output power, efficiency and maximum switch voltage and current follow.

\subsubsection{Case study: high power, high efficiency Class-E PA un- der antenna load-mismatch conditions}

For high output power and high efficiency, a class-E PA is conventionally designed to have $q=1.412,50 \%$ duty cycle $(d=1)$, ZVS $(\alpha=0)$ and ZSS $(\beta=0)[20]$. We assume a lossless reciprical matching network that matches the nominal load impedance for this PA to a nominal antenna impedance. The nominal K-design set elements and the nominal PA performance metrics $P_{\text {out }, \text { nom }}, \eta_{\text {nom }}$, the maximum switch voltage and the maximum switch current at nominal conditions are shown in Table 4.2 for two different values of switch on-resistance, as defined in (4.5), and $Q_{L}$. The performance of the class-E PAs for the two different switch on-resistances and two different $Q_{L} \mathrm{~S}$ as listed in Table 4.2 are examined below.

Due to the complexity of the equations (4.31) and (4.32), a closed form equations for $\alpha$ and $\beta$ are not derived in this chapter (if they can be derived at all). Instead, for the design parameters in Table 4.2, we use graphical representations and discuss the effect of the parameters $m$ and $Q_{L}$. Using a numerical solver to solve (4.31) and 
Table 4.2: Design parameters and performance metrics for different switch onresistances and $Q_{L}$ s.

\begin{tabular}{lccc}
\hline & $m=0$ & $m=0.05$ & $m=0.05$ \\
& $Q_{L} \rightarrow \infty$ & $Q_{L} \rightarrow \infty$ & $Q_{L}=25$ \\
\hline$K_{L_{\text {nom }}}$ & 0.73 & 0.7 & 0.65 \\
\hline$K_{C_{\text {nom }}}$ & 0.68 & 0.72 & 0.77 \\
\hline$K_{X_{\text {nom }}}$ & 0 & 0.09 & 0.11 \\
\hline$K_{P_{\text {nom }}}$ & 1.36 & 0.977 & 0.91 \\
\hline$\eta_{\text {nom }}$ & $100 \%$ & $80.3 \%$ & $72.8 \%$ \\
\hline$\frac{V_{C m_{\text {max }}}}{V_{D D}}$ & 3.65 & 3.29 & 3.18 \\
\hline$\frac{I_{\text {smax }}}{I_{\text {nom }_{\text {nom }}}}$ & 2.65 & 2.68 & 2.69 \\
\hline
\end{tabular}

(4.32), constant $\alpha$ and $\beta$ contours for $\mathrm{VSWR}_{Z_{A}}<20$ are shown in Fig. 4.3 where each Smith chart corresponds to a different value for $m$ and $Q_{L}$. In all Smith charts, the center points correspond to the nominal load impedance and (for this case study) to $\alpha=\beta=0$. For illustration purposes, the circles for $\mathrm{VSWR}_{Z_{A}}=2, \mathrm{VSWR}_{Z_{A}}=5$, $\mathrm{VSWR}_{Z_{A}}=10$ and $\mathrm{VSWR}_{Z_{A}}=20$ are also shown in Fig. 4.3.

Fig. 4.3 shows that for a loss-less switch and $Q_{L} \rightarrow \infty$ the value of $\alpha$ can change between -7 and 7 for $\operatorname{VSWR}_{Z_{A}} \leq 10$, while the value of $\beta$ is in the range of -2.3 to 21. This implies that ZVS and ZSS conditions are heavily violated with increasing $\operatorname{VSWR}_{Z_{A}}$. With increasing the switch loss (increasing $m$ ) and dc-inductor loss (reducing $Q_{L}$ ) the ranges of $\alpha$ and $\beta$ reduce.

Below, the effect of load-mismatch on output power, efficiency and maximum switch voltage and current are presented. This is done using the theory in the previous sections that assumes a loaded $Q_{\text {loaded }} \rightarrow \infty$, and is backed up by circuit simulations using ideal components where we used a simple LC filter $\left(L_{0}\right.$ and $C_{0}$ in Fig.4.1(a)) at the output with $Q_{\text {loaded }}=5$ to demonstrate the effect of a limited loaded $\mathrm{Q}$ filter. At much higher $Q_{\text {loaded }}$, simulation results and theoretical results match exactly.

In the simulations, a simple series RLC network was used to create the loadmismatch for the PA. Then, in the equation $Z^{\prime}=k R+j k^{\prime} R$, given by $(4.23)$, for any load-mismatch (any $k$ and $k^{\prime}$ ) the real part of the PA load at all harmonics of the operating frequency is the same while for the inductive (capacitive) mismatch, the imaginary part of the load scales up by $n$ (down by $n$ ) at the $n^{\text {th }}$ harmonic. Appendix A deals with the effect of PA impedance at the harmonics of the operating frequency on the PA performance in more detail.

Components values, shown in Fig. 4.1, are calculated according to K-design set 


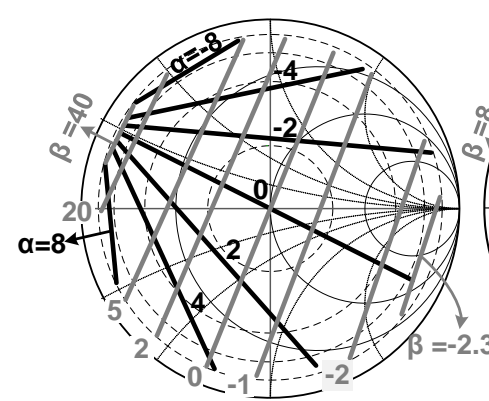

a) $\mathrm{m}=\mathbf{0 , \mathrm { Q } _ { \mathrm { L } } \rightarrow \text { Inf. }}$

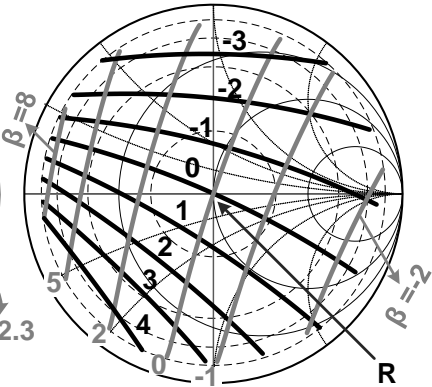

b) $\mathrm{m}=0.05, \mathrm{Q}_{\mathrm{L}} \rightarrow$ Inf.

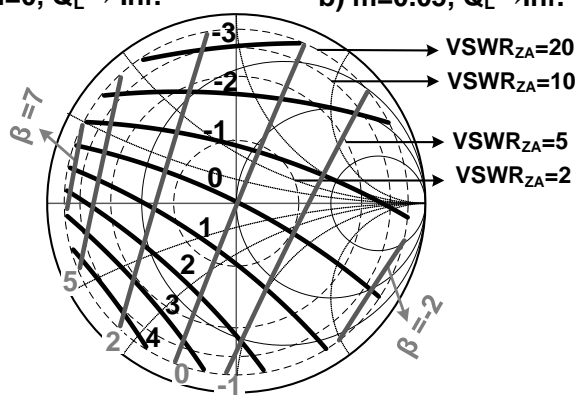

c) $m=0.05, Q_{L}=25$

Figure 4.3: Constant $\alpha$ (black) and $\beta$ (grey) contours for the different values of $m$ and $Q_{L}$ in the $\Gamma_{Z^{\prime}}$ plane (cross section BB in Fig. 4.2).

elements in Table 4.2 for $R=20 \Omega, V_{D D}=1.2 \mathrm{~V}$ and $\frac{\omega_{0}}{2 \pi}=1.4 \mathrm{GHz}$ and are listed in Table 4.3 .

Table 4.3: Components values and simulated output power for $q=1.412, d=1$, $R=20 \Omega, V_{D D}=1.2 \mathrm{~V}$ and $\frac{\omega_{0}}{2 \pi}=1.4 \mathrm{GHz}$.

\begin{tabular}{cccccc}
\hline & $L$ & $C$ & $X$ & $R_{\text {on }}$ & $P_{\text {out }, \text { nom }}$ \\
\hline$m=0, Q_{L} \rightarrow \infty$ & $1.7 \mathrm{nH}$ & $3.9 \mathrm{pF}$ & 0 & 0 & $19.9 \mathrm{dBm}$ \\
\hline$m=0.05, Q_{L} \rightarrow \infty$ & $1.6 \mathrm{nH}$ & $4.1 \mathrm{pF}$ & $0.2 \mathrm{nH}$ & $1.4 \Omega$ & $18.5 \mathrm{dBm}$ \\
\hline$m=0.05, Q_{L}=25$ & $1.5 \mathrm{nH}$ & $4.4 \mathrm{pF}$ & $0.25 \mathrm{nH}$ & $1.3 \Omega$ & $18.2 \mathrm{dBm}$ \\
\hline
\end{tabular}

\section{Output power}

Using (4.30), the output power of the PA under load-mismatch conditions normalized to $P_{\text {out }, \text { nom }}$ is

$$
\frac{P_{\text {out }}}{P_{\text {out }, \text { nom }}}=\frac{1}{k} \frac{K_{P}}{K_{P_{\text {nom }}}}
$$


With (4.22) and (4.26), load-pull output power contours can be derived; these contours are shown in Fig. 4.4 with solid lines for two values of $m$ and $Q_{L}$. Fig. 4.4(a) shows that for a lossless switch $(m=0)$ and ideal dc-feed inductor, for $\mathrm{VSWR}_{Z_{A}}$ up to 10 the output power can change by almost $\pm 10 \mathrm{~dB}$. Increasing $m$ from 0 to 0.05 , Fig. 4.4 (b), for $Q_{L} \rightarrow \infty$ changes this variation from about $-8.5 \mathrm{~dB}$ to $+2.4 \mathrm{~dB}$. Reducing $Q_{L}$ to 25 further reduces this range (Fig. 4.4(c)). Simulation results with ideal components and assuming $Q_{\text {loaded }}=5$ for the nominal load impedance are shown in Fig. 4.4 with dashed lines. Note that e.g. at the right hand side of the Smith charts the $Q_{\text {loaded }}$ is much lower than in the nominal case. Fig. 4.4 demonstrates good agreement between the theory and the simulation results.

\section{Efficiency}

Load-pull power efficiency contours obtained from (4.20) are shown in Fig. 4.4(d-f) by solid curves. It can be seen that power efficiency drops from $100 \%$ to about $35 \%$ for $m=0, Q_{L} \rightarrow \infty$ and $\mathrm{VSWR}_{Z_{A}}=10$. The power efficiency reduction using a lossy switch can be even worse: for $\operatorname{VSWR}_{Z_{A}}=10$ and $m=0.05$ the efficiency can be reduced to less than $25 \%$.

Two important observation can be made from Fig. 4.3 and 4.4. Firstly, the loadpull contour for $\alpha=0$ shows that it is possible to sweep output power while keeping the efficiency high (100\% in case of a lossless switch and an ideal dc-feed inductor). Secondly, ZVS and ZSS conditions do not necessarily provide maximum efficiency in case of a lossy switch [13]. Fig. 4.4(e) shows that by choosing the right values for $\alpha$ and $\beta$ for $m=0.05$ and $Q_{L} \rightarrow \infty$, the power efficiency can be increased up to $88 \%$ where the efficiency is about $80 \%$ under ZVS and ZSS conditions.

Fig. 4.4(e) and (f) show that reducing $Q_{L}$ from $\infty$ to 25 reduces the efficiency for the both nominal and non-nominal loads; for $m=0.05$ and the nominal load, efficiency is reduced from $80.3 \%$ to $72.8 \%$. Having the load-pull contours for the output power, shown in Fig. 4.4(b) and (c), on top of the corresponding efficiency contours, shown in Fig. 4.4(e) and (f), shows that at $10 \mathrm{~dB}$ back-off, more than $80 \%$ efficiency can be obtained for $Q_{L} \rightarrow \infty$ while $Q_{L}=25$ reduces the $10 \mathrm{~dB}$ back-off efficiency to slightly more than $40 \%$.

Simulation results with ideal components and $Q_{\text {loaded }}=5$ under nominal load condition are also shown in Fig. 4.4(d-f) with dashed curves. At the right hand side of the Smith charts the $Q_{\text {loaded }}$ is much lower than in the nominal case, which yields significant transmission of higher harmonics. Because power efficiency is derived for the fundamental harmonic, there the power efficiency decreases with lower $Q_{\text {loaded }}$. For example for $m=0$ and load-mismatch $\Gamma^{\prime}=0.82\left(\mathrm{VSWR}_{Z_{A}}=10\right)$ the calculated and simulated efficiencies are $66 \%$ and $56 \%$, respectively. For $Q_{\text {loaded }}=10$ the power transmission at the second and third harmonics is lower and the simulated 


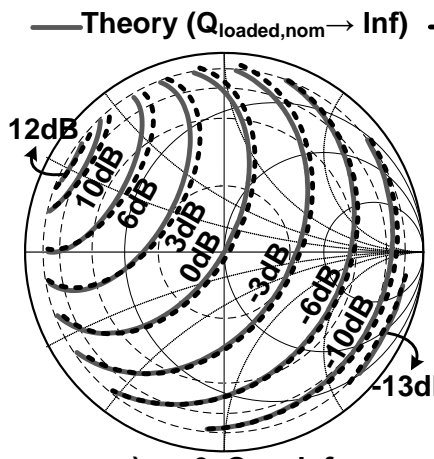

a) $\mathrm{m}=0, Q_{\mathrm{L}} \rightarrow$ Inf.

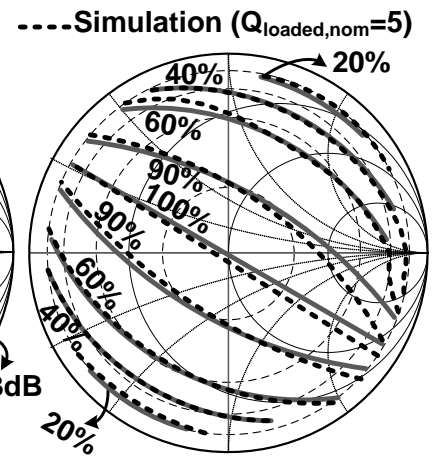

d) $m=0, Q_{L} \rightarrow$ Inf.

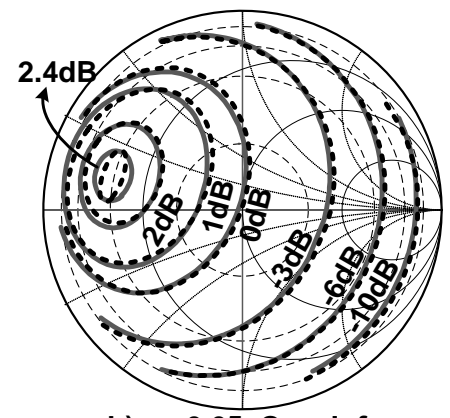

b) $\mathrm{m}=0.05, \mathrm{Q}_{\mathrm{L}} \rightarrow$ Inf.

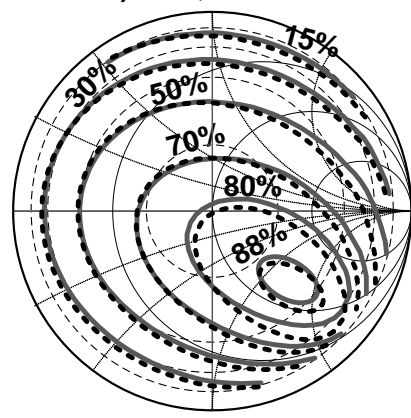

e) $\mathrm{m}=0.05, \mathrm{Q}_{\mathrm{L}} \rightarrow$ Inf.
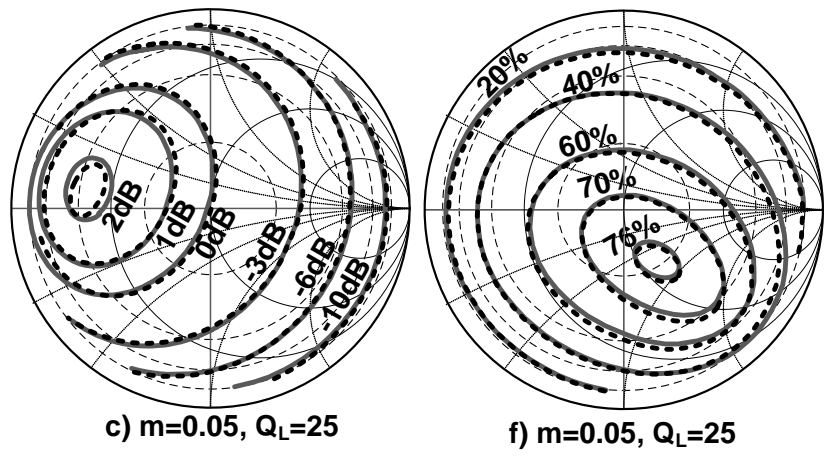

Figure 4.4: Load-pull contours for output power normalized to nominal condition (a-c) and efficiency (d-f), for different values of $m$ and $Q_{L}$ in the $\Gamma_{Z^{\prime}}$ plane. 
efficiency increases to $60 \%$ and for $Q_{\text {loaded }} \rightarrow \infty$ the simulated efficiency approaches the calculated $66 \%$ efficiency.

\section{Maximum and minimum switch voltage}

When the switch is off $\left(d \pi<\omega_{0} t<2 \pi\right)$, the maximum switch voltage contours ${ }^{3}$ are shown in Fig. 4.5. For VSWR $=10, Q_{L} \rightarrow \infty$ and $m=0$, the switch voltage increases from nominally $3.65 V_{D D}$ up to $13 V_{D D}$. A lossy inductor and/or a lossy switch reduces this high maximum voltage (at the cost of power and efficiency); with $Q_{L}=25$ and $m=0.05$, the switch voltage increases up to (slightly more than) $5 V_{D D}$ for VSWR $=10$. Calculated time domain switch voltage waveforms for three different cases are also shown in Fig. 4.5. Simulation results assuming a nominal $Q_{\text {loaded }}=5$ are represented by dashed curves in Fig. 4.5: using $Q_{\text {loaded }}=5$ slightly increases the maximum switch voltage compared to using $Q_{\text {loaded }} \rightarrow \infty$.

Fig. 4.5 also shows that for some load impedance ranges, the switch voltage reaches negative values (up to $-10 V_{D D}$ for $m=0$ ). The theoretical and simulated minimum switch voltage for 2 different cases are shown in Fig. 4.6. In circuit implementations, this may e.g. forward bias parasitic diodes which in turn may inject current into the substrate, yields significant gradients in the substrate potential across the chip and yielding latch up risks that may destroy the PA operation [34], [35] when improperly handled. Circuit solutions such as used in e.g. [36] may be used to reliably handle these large negative voltages at the cost of circuit complexity and power efficiency. Section 4.6 deals in more detail with the effects of (unintentially - due to load-mismatch) forward biasing of e.g. parasitic diodes on the behavior of SMPAs.

\section{Maximum and average switch current}

When the switch is on $\left(0<\omega_{0} t<d \pi\right)$, the maximum switch current normalized to the maximum switch current at nominal load condition ${ }^{4}$ can be written as

$$
\frac{I_{\text {smax }}}{I_{\text {smax }, \text { nom }}}=\frac{\frac{I_{\text {smax }}}{I_{0}}}{\frac{I_{\text {smax }, \text { nom }}}{I_{0, \text { nom }}}} \frac{I_{0}}{I_{0, \text { nom }}} \text {. }
$$

In (4.34), $\frac{I_{s \max }}{I_{0}}$ under mismatch conditions follows from $(4.19), \frac{I_{s \max , \text { nom }}}{I_{0, \text { nom }}}$ is listed in Table 4.2 and $\frac{I_{0}}{I_{0, \text { nom }}}$ is the ratio of the average switch/supply current (note that $\left.<i_{s}(t)>=<i_{L}(t)>=I_{0}\right)$ under mismatch conditions to that at the nominal load condition. Using $\eta=\frac{P_{\text {out }}}{V_{D D} I_{0}}$ it follows that

$$
\frac{I_{0}}{I_{0, \text { nom }}}=\frac{P_{\text {out }}}{P_{\text {out }, \text { nom }}} \frac{\eta_{\text {nom }}}{\eta}
$$

\footnotetext{
${ }^{3}$ It is a common practice to normalize maximum switch voltage to the supply voltage $V_{D D}$.

${ }^{4}$ To keep the analysis independent from the output power, the maximum switch current is normalized to that at nominal load condition.
} 


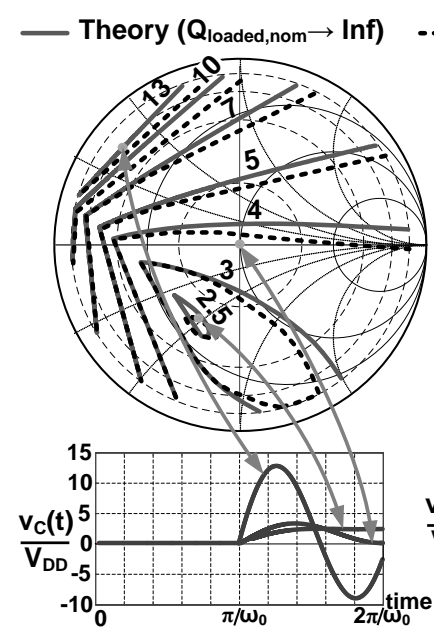

a) $m=0, Q_{L} \rightarrow$ Inf.

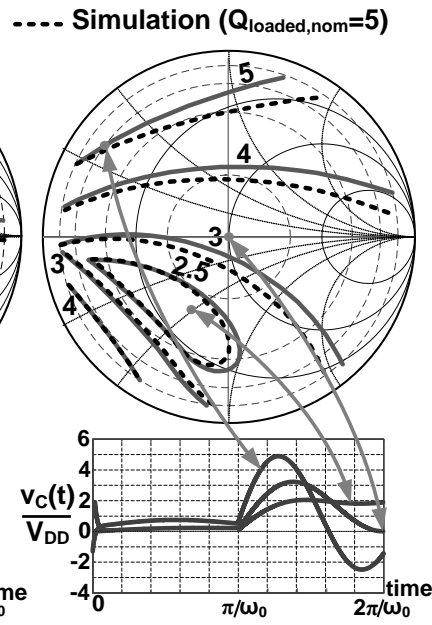

b) $m=0.05, Q_{L}=25$

Figure 4.5: Maximum switch voltage of class-E PA normalized to $V_{D D}$ for different values of $m$ and $Q_{L}$ in the $\Gamma_{Z^{\prime}}$ plane.

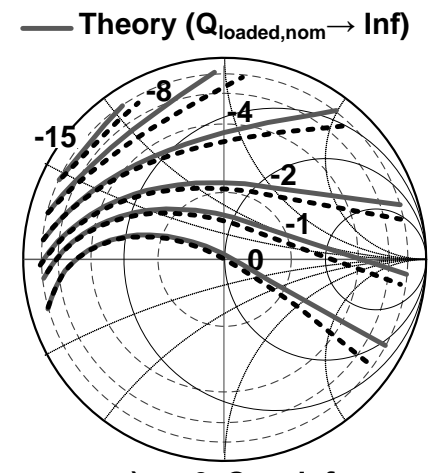

a) $m=0, Q_{L} \rightarrow$ Inf.

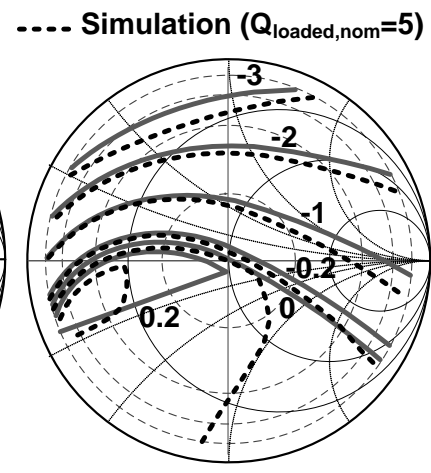

b) $m=0.05, Q_{L}=25$

Figure 4.6: Minimum switch voltage of class-E PA normalized to $V_{D D}$ for different values of $m$ and $Q_{L}$ in the $\Gamma_{Z^{\prime}}$ plane. 
The $\frac{I_{\text {smax }}}{I_{\text {smax }, \text { nom }}}$ load-pull contours using (4.34) and (4.35) are shown in Fig. 4.7. For small $m$ and non-zero voltage switching $(\alpha \neq 0)$, the maximum current occurs at $t=0^{+}$. Fig. 4.7(a) shows the maximum switch current neglecting current spikes at the switching moment. Antenna load-mismatch with VSWR $=10$, can increase the maximum switch current up to (almost) 3 times that under nominal load conditions. This ratio increases for a higher $Q_{L}$ or a lower $m$; up to 9 times for $m=0$ and $Q_{L} \rightarrow \infty$. The normalized current spike at switching, in Fig. 4.7(b), shows that for $m=0.05$ and $Q_{L}=25$ the switching moment current can be 15 times as high as the maximum switch current in the nominal load condition. This ratio can be even higher for smaller values of $m$. Simulation results with $Q_{\text {loaded }}=5$ under nominal load condition are shown in Fig. 4.7 as dashed curves.

The load-pull contours for the average switch/supply current under load-mismatch conditions normalized to that under nominal conditions are obtained from (4.35) and shown in Fig. 4.8 for $m=0.05$ and $Q_{L}=25$. The difference between the theoretical results and the simulation results at the right hand side of the Smith chart is due to the much lower $Q_{\text {loaded }}$ at this region of the Smith chart compared to the nominal load condition. It can be seen that toward the left side of the Smith chart, the average switch/supply current increases. For $m=0$ and $Q_{L} \rightarrow \infty$, the ratio can be up to 10 for $\operatorname{VSWR}=10$.

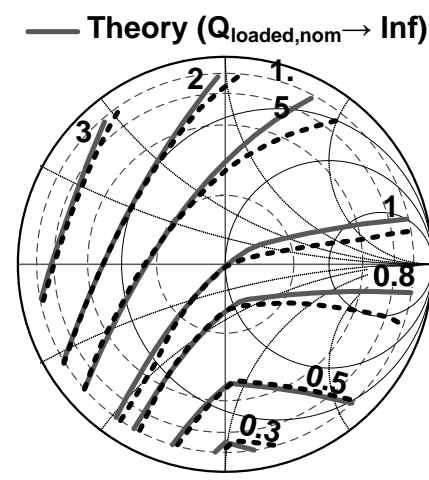

(a)

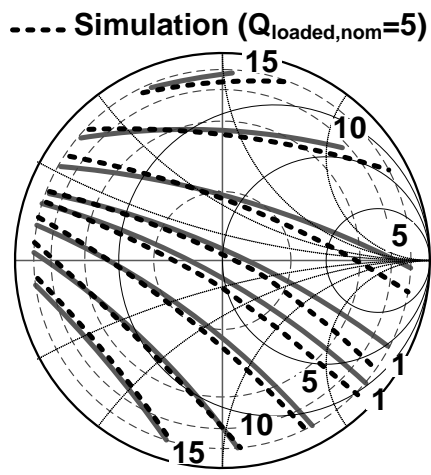

(b)

Figure 4.7: (a) Maximum switch current of class-E PA, neglecting the switching moment, normalized to nominal condition. (b) Switching moment current normalized to the maximum switch current at nominal condition. For both plots $m=0.05$ and $Q_{L}=25$. 


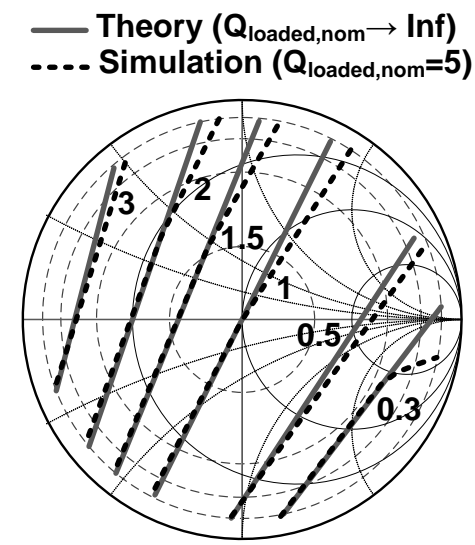

Figure 4.8: Average switch/supply current of class-E PA normalized to that at nominal load condition for $m=0.05$ and $Q_{L}=25$.

\subsection{Reliability issues and safe operating region}

For a transistor operating in a class-E PA and designed to marginally handle the nominal switch voltage and switch current, high voltage or current stress will drastically reduce the life time [37]. Figs. 4.5 and 4.7 already showed that under load-mismatch conditions both the switch voltage and switch current can increase significantly with respect to the nominal load condition. A discussion into reliability and safe operating areas for class-E PAs is shown below.

Two load-pull contours both of maximum switch voltage normalized to $V_{D D}$ and of maximum switch current normalized to that at nominal load condition are shown in Fig. 4.9 with solid and dashed lines, respectively. For this plot, it is assumed that the nominal PA is designed to have $q=1.412, d=1, m=0, Q_{L} \rightarrow \infty$ and $\alpha=\beta=0$, similar to the case study in section 4.4.3. Fig. 4.9 also shows two greyed regions. The inner (light grey) region is bounded by the $V_{c \max }=3.65 V_{D D}$ contour and the $I_{\text {smax }}=I_{\text {smax }, \text { nom }}$ contour; this region represents the impedances for which the maximum switch voltage and current are lower than those for the nominal load, corresponding to the safe operating region with $0 \%$ design margin. Adding $10 \%$ margin in both $V_{\text {cmax }}$ and $I_{\text {smax }}$ adds the dark grey region to the previously described safe operating region for $0 \%$ design margin. To prevent device performance degradation and life time issues, the impedance outside of the greyed areas should be avoided. Since the switch current at the switching moment depends heavily on the technology used and on the exact waveform of the driving voltage, it is not taken into account for the safe operating region, otherwise the safe operating region would have been smaller. Including the switch conduction loss (non-zero $m$ ) and dc-feed inductor loss (limited $Q_{L}$ ), the safe operating region can be extended at the cost of output 
power and efficiency, as discussed in section 4.4 .

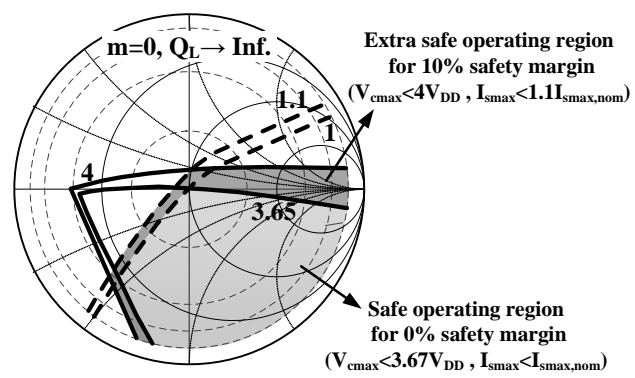

Figure 4.9: Solid contours show maximum switch voltage normalized to $V_{D D}$ and dashed contours show maximum switch current normalized to that at the nominal condition (for $m=0$ and $Q_{L} \rightarrow \infty$ ). The light grey area denotes the safe operating region with $0 \%$ safety margin. The dark grey area corresponds to the extra safe operating region for $10 \%$ design margin.

The performance and the reliability related parameters of class-E PAs under loadmismatch conditions were shown for $q=1.412$ and $d=1$. It is possible to adjust duty cycle $d$ or parameter $q$ to satisfy ZVS and/or ZSS conditions at different frequencies [38] or at different output power levels [30]. The next section shows the effect of loadmismatch for different duty cycles and a short discussion on the effects of different $q$ parameter.

\subsubsection{Effects of the parameters $d$ and $q$}

Output power and efficiency contours for a class-E PA (which is initially designed) for $d=0.75$ and $d=1.4$ are shown in Fig. 4.10, for $q=1.412$. The corresponding load-pull contours for a $50 \%$ duty cycle $(d=1)$ were shown in Fig. 4.4. In Fig. 4.10 it is assumed that $m=0, Q_{L} \rightarrow \infty$ and that $\alpha=\beta=0$ for the nominal load condition.

It can be seen that the shape of the power and efficiency contours is not much affected by the value of $d$ but that the contours do rotate counterclockwise for increasing $d$. Simulation results with $Q_{\text {loaded }}=5$ for the nominal load impedance are shown as dashed curves in Fig. 4.10. The relatively large differences between theory $\left(Q_{\text {loaded }} \rightarrow \infty\right)$ and the simulations are due to a significant dependency of the harmonic content in the output signal to the load impedance (At much higher $Q_{\text {loaded }}$, simulation results and theoretical results match exactly). An in-depth analysis is not provided here for length reasons.

Fig. 4.11 shows the maximum normalized switch voltage and current for $d=0.75$ and $d=1.4$; the corresponding load-pull plots for $d=1$ are shown in Fig. 4.5 and Fig. 4.7 respectively. The grey areas again denote the safe operating regions with $0 \%$ 


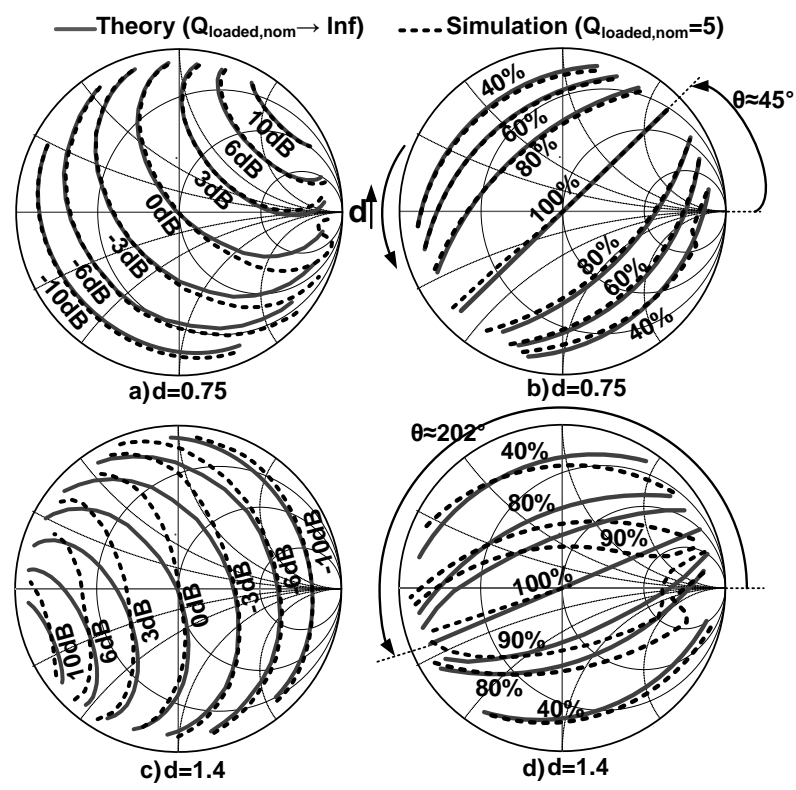

Figure 4.10: (a,c) Normalized output power contours and (b,d) power efficiency contours for $d=0.75$ and $d=1.4$. The switch and the dc-feed inductor are ideal.

(light grey) and 10\% (light grey + dark grey) safety margin, that are enclosed by the corresponding maximum voltage and current contours. It can be seen that a higher $d$ reduces the safe operating region and rotates it counterclockwise.

Similar contours can be plotted for different values of $q$. It can be shown that the shape of the contours hardly changes except for a rotation: clockwise rotation for higher $q[19]$. These contours are not shown here for the length reason.

\subsection{Impact of non-ideal effects}

The previous section presented load-pull analyses and the impact of load-mismatch on output power, efficiency and reliability, primarily from a mathematical point of view; these theoretical findings are exact for a loaded $Q_{\text {loaded }} \rightarrow \infty$. These theoretical findings were backed up by simulations that assumed a $Q_{\text {loaded }}=5$ for nominal load condition, already showing the impact of finite $Q_{\text {loaded }}$. Other non-idealities that were already included were non-zero switch on-resistance, modelled by $m \neq 0$ and non-ideal dc-feed inductor, modelled by a limited $Q_{L}$. In this section, we briefly analyze the main remaining non-idealities from a load-mismatch point of view: a) reverse conduction of the switch (or shunting negative voltage excursions across the switch), b) load dependent switch conduction loss $(m)$. 


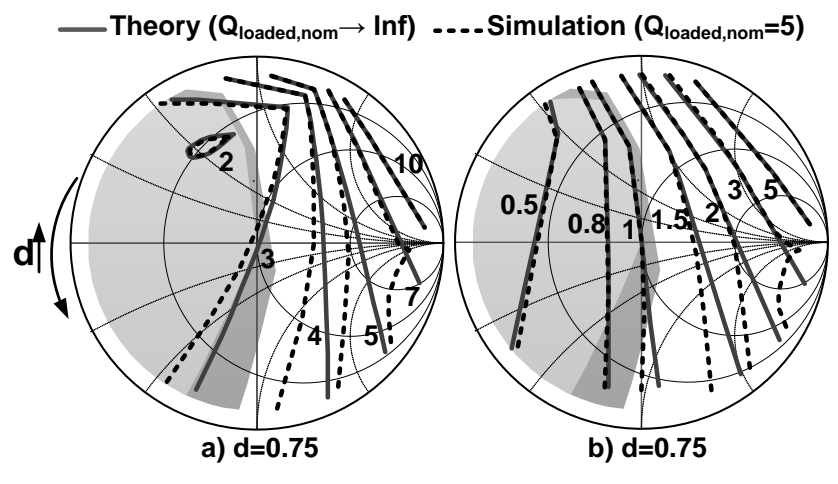

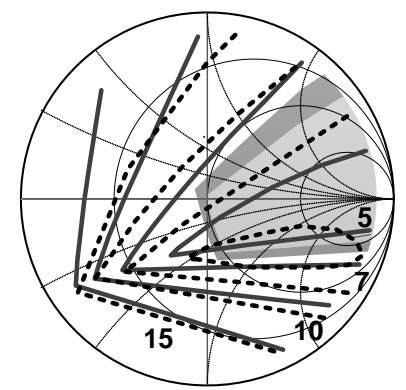

c) $d=1.4$

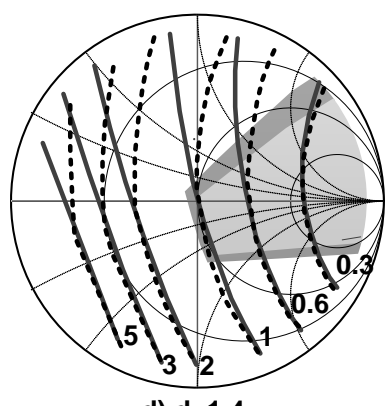

d) $d=1.4$

Figure 4.11: (a,c) Maximum switch voltage normalized to $V_{D D}$ and (b,d) maximum switch current normalized to that at nominal condition for $d=0.75$ and $d=1.4$. The switch is ideal $(\mathbf{m}=0)$ and $Q_{L} \rightarrow \infty$. The light grey area denotes the safe operating region with $0 \%$ safety margin. The dark grey area corresponds to the extra safe operating region for $10 \%$ design margin.

\subsubsection{Shunting negative voltage excursions across the switch}

To show the impact of the limitation of the negative excursion of the switch voltage, let us assume a class-E PA with ideal switch $(\mathrm{m}=0)$ and ideal dc-feed inductor that was studied in section 4.4.3. Fig. 4.6(a) shows load-pull contours for the minimum switch voltage. It can be seen that in theory the switch voltage can go to large negative values: e.g. to $-8 V_{D D}$ for load-mismatch $\Gamma^{\prime}=0.82 \angle 151^{\circ}$ when $m=0$ and $Q_{L} \rightarrow \infty$.

In actual circuit implementations, large negative voltage excursions across the switch transistor usually cannot occur due to two effects. Firstly, the parasitic diode across the switch (e.g. the drain-bulk diode in bulk CMOS technologies), such as shown in Fig. 4.12(a), is in forward for sufficiently negative voltages. Secondly, for sufficiently negative switch voltages the switch transistors typically goes into conduction forming e.g. a MOS diode for MOS switches (with source and drain interchanged). 
Both of these diodes can limit negative voltage excursions; usually one of the two is dominant (depending on $V_{t h}$ ). The work in [16] explicitly adds an anti-parallel diode to prevent negative voltage excursions across the switch (at the switching moment) aiming to have (ideally) zero switching loss $(\alpha=0)$ under specific (real) PA load variations.

a)

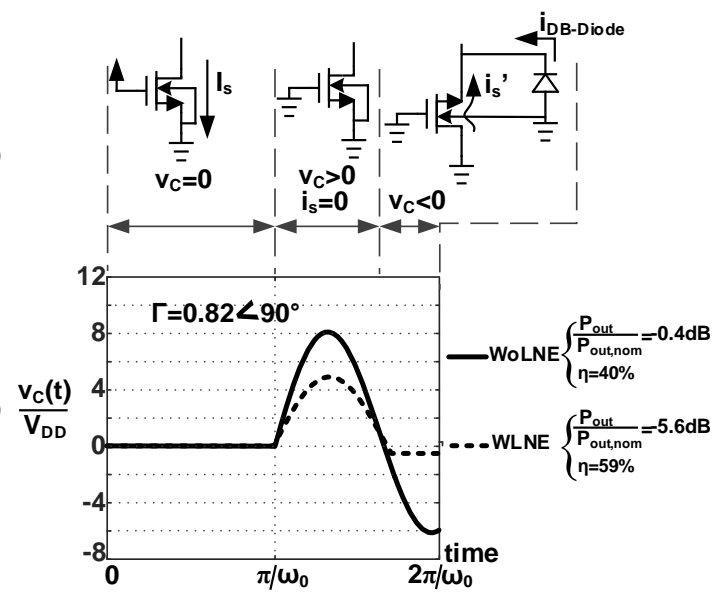

b)

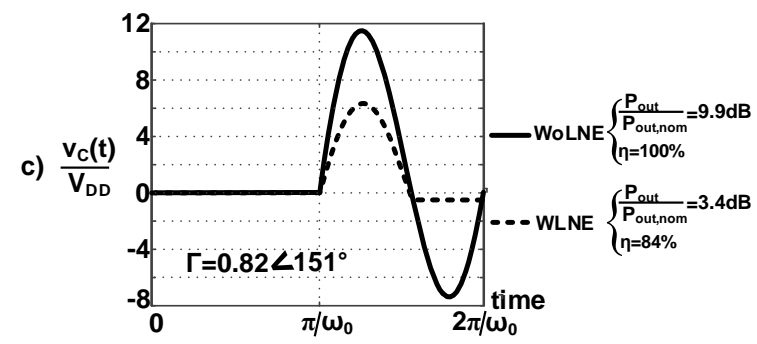

Figure 4.12: (a) Switch model for positive and negative voltages. (b,c) Switch voltage, output power and efficiency for two different load-mismatches with limited negative excursions (WLNE) and without limited negative excursions (WoLNE).

Assuming that the negative voltage excursions are hard-limited to e.g. $-0.5 V_{D D}$ by one of the limiting mechanisms described above, the simulated switch voltage, with and without the limited negative voltage excursions (WLNE and WoLNE, respectively), for two load-mismatches $\Gamma^{\prime}=0.82 \angle=90^{\circ}$ and $\Gamma^{\prime}=0.82 \angle=151^{\circ}$ are shown in Fig. 4.12(b) and Fig. 4.12(c), respectively. In these figures, also output power levels and efficiencies are reported for the 4 cases. Three observations can be made. Firstly, the limitation on the negative voltage increases the effective duty cycle. Secondly, this can either reduce or increase power efficiency. Increased efficiency is due to reduced switching loss (smaller $\alpha$ ) while the efficiency reduction results from 
conduction loss in the diode. Thirdly, the maximum switch voltage and output power (and therefore switch current) are influenced.

\subsubsection{Load dependent switch conduction loss $(m)$}

To simplify the analyses in sections 4.3 and 4.4, the switch conduction loss was assumed to be constant during the time period that the switch was closed. In section 4.3 , the switch current was shown to depend on the switching conditions (and as a result on the PA load). Also, in Fig. 4.8, the average switch current under loadmismatch conditions normalized to that at nominal load condition was shown to be load-dependent. This load-dependent average switch current results in a loaddependent average switch voltage across the switch during the time period that the switch is closed $\left.\left(<v_{C \text {,on }}\right\rangle\right)$. Implemented by (MOS) transistors, the average switch on-resistance depends on both the drive (gate-source) voltage as well as on the switch on-voltage (drain-source voltage) [34]. Therefore, the switch on-resistance changes under load-mismatch.

For switches that employ a cascode structure with a fixed gate bias for the cascode device, the switch on-resistance change can be severe. In this case the increased average switch on-voltage of the bottom transistor reduces the average gate-source voltage of the cascode device which increases the average switch on-resitance. Accurate modeling of the average switch on-resistance is beyond the scope of this thesis work. However, for the switch we implemented, see section 4.7, the average switch on-resistance is estimated for different PA loads from circuit simulations.

\subsubsection{Load-pull contours}

Now, combining these findings with the results from sections 4.4 and 4.5.1 yields the effect of load variations on SMPA behavior from a theoretical point of view, including shunting negative voltage excursions and including the load-dependent $m$. For that purpose, the simplified model shown in Fig. 4.1(a) is considered with $d=1$ and $m=0.05$ for nominal conditions as starting point. Then, the effective duty cycle and the effective $m$ are estimated by fitting the load-pull simulations of the implemented PA in $65 \mathrm{~nm}$ CMOS technology to the corresponding theoretical counterparts resulting from our model; for a limited yet enough number of PA loads, the parameters $m$ (in the range of 0.05 to 0.1 with 0.005 step) and $d$ (in the range of 1 to 1.4 with 0.025 step) were adjusted in our theoretical plots to have the best match between the simulated performance metrics (power and efficiency) and reliability related metrics (switch voltage waveform) of the implemented PA and these metrics as resulting from the theory. The resulting effective $d$ and $m$ contours are shown in Fig. 4.13(a) and (b), respectively. 
In Fig. 4.13(a), it was assumed that the negative voltage excursions are hardlimited to e.g. $-0.5 V_{D D}$ (corresponding to the contour $d_{\mathrm{eff}}=1$ ). In the lower part of the Smith chart (below the contour $d_{\text {eff }}=1$ ), the minimum switch voltage is higher than $-0.5 V_{D D}$ and hence the effective duty cycle is equal to the nominal duty cycle $d=1$. Towards the upper left corner on the Smith chart, the switch voltage tends to go more negative, see Fig. 4.6, resulting in the effective $d$ contours in Fig. 4.13(a). With the results from section 4.5.1, this yields anti-clockwise rotation of power, efficiency and maximum voltage and current contours in only the parts on Smith chart where $d_{\text {eff }}>1$.

The effective $m$ contours are shown in Fig. 4.13(b). Toward the upper and left side of the Smith chart the effective $m$ increases due to increased losses with longer forward biasing of the parasitic diode (or reverse conduction of the switch with a higher loss in reverse mode) and increased average switch current, shown in Fig. 4.8. At the right and lower part of the Smith chart (below the contour $m_{\text {eff }}=0.05$ ), the effective $m$ is equal to its nominal value $m=0.05$.

These effective $d$ and $m$ contours yield the load-pull plots in Fig. 4.13(c-f). In the load-pull plots, the solid curves are for the SMPA including the limited negative voltage and the load-dependent $m$; the dotted contours assume fixed parameters $d=1$ and $m=0.05$ for all the PA loads, already shown in section 4.4.3. Fig. 4.13(c) and (d) show that, toward the upper left corner of the Smith chart, the power contours with considering the limited negative voltage rotate in counter-clockwise direction and the efficiency is higher than for the case that $d=1$ and $m=0.05$. Toward the left side of the Smith chart, the increased switch condition loss reduces the output power as well as the efficiency compared to case where $m$ is fixed at 0.05 .

Fig. 4.13(e) and (f) show that the anti-clockwise rotation of the contours reduces both the maximum switch voltage and the average current toward the upper left corner on the Smith chart which results in an extended safe operating region. Also, the reduced maximum switch voltage at the left side of the Smith chart for solid contours is due to the increased switch conduction loss. Since, in the simulation of the implemented class-E PA as well as in the measurements, distinguishing between resistive and capacitive current of the switch is not possible, no contours for the maximum switch current are shown.

\subsection{Measurements}

Finally, to show the correspondence between theoretical findings, simulations of idealized SMPAs and measurements of an actual SMPA, this section presents measurement results of a circuit implemented in a standard $65 \mathrm{~nm}$ CMOS technology. The 


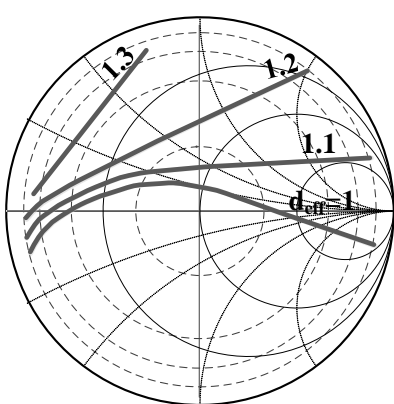

(a)

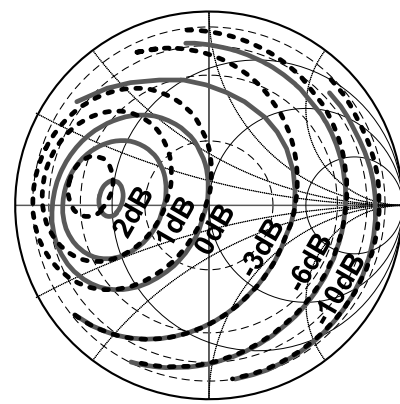

(c)

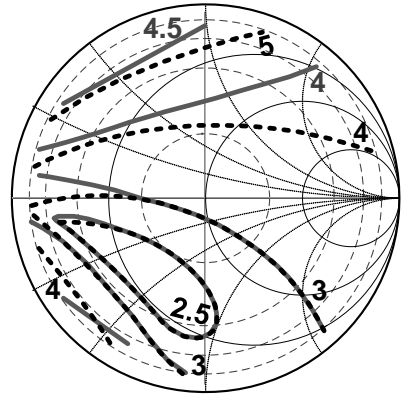

(e)

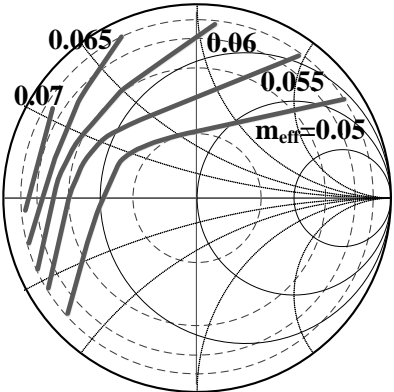

(b)

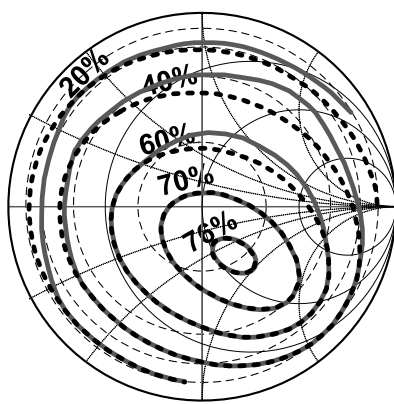

(d)

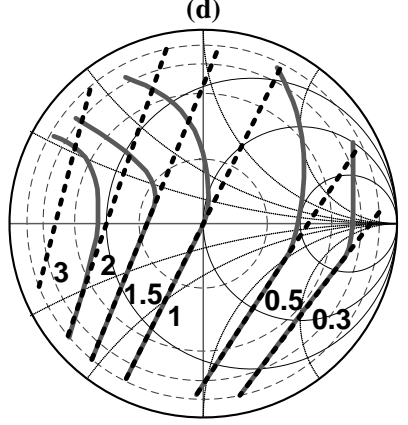

(f)

Figure 4.13: Load-pull contours with (solid contours) and without (dotted contours) taking the limited negative voltage and load-dependent $m$ effects into account: (a) estimated $\mathbf{d}$ when negative switch voltage is hard-limited to $-0.5 V_{D D}$, (b) estimated $\mathrm{m}$, (c) normalized output power, (d) efficiency, (e) maximum switch voltage normalized to $V_{D D}$, (f) average switch/supply current normalized to that under nominal condition. 
schematic of the class-E PA and driver ${ }^{5}$ stage are shown in Fig. 4.14. The switch is implemented as a cascode structure employing a $1.2 \mathrm{~V}$ device as the switch transistor and a thick oxide $2.5 \mathrm{~V}$ device as the cascode allowing switch voltages up to $4 \mathrm{~V}$. The $\mathrm{PA}$ is designed for $q=1.412, d=1$ and $\alpha=\beta=0$ under nominal load condition $R_{L}=20 \Omega$. The feed inductance $L \approx 1.5 \mathrm{nH}$ (see Table 4.3 ) is implemented by 2 parallel bond-wires to provide a high quality factor $\left(Q_{L} \approx 25\right)$. The tank capacitance $C \approx 4.4 \mathrm{pF}$ at the switching node was implemented with the drain-bulk and gate-drain capacitances of the NMOS transistor. Also, a switch-capacitor network was added to the switching node to (fine) tune the drain capacitance to the required value. The output filter $\left(L_{0}-C_{0}\right)$ and the matching network $\left(L_{m}-C_{m}\right)$ are implemented on the PCB; the total required inductance $L_{0}+L_{m} \approx 14 \mathrm{nH}$ is partly implemented by bond-wire inductances and partly by an off-chip discrete component. The matching network steps the $50 \Omega$ antenna load down to $20 \Omega$. The output filter provides a loaded quality factor $Q_{\text {loaded }} \approx 5$ under nominal load condition. The output of the matching network is connected to a Maury load tuner, capable of providing VSWR up to 40 at cross section AA. Due to the loss of the connector cable, the matching network and the output filter, the VSWR is limitted at cross section BB to 6.15 and 10 at the left and right hand sides of the Smith chart, respectively. All PA load settings for the measurements at cross section BB, are shown in Fig. 4.14. For each of these settings the output power, efficiency and time domain switch voltage are measured. Our presented load-pull contours are based (interpolated) on these settings.

Measured load-pull contours for normalized output power are shown in Fig. 4.15(a) together with theoretical contours for $m=0.05$ and $Q_{L}=25$ (with taking the limited negative voltage and the load-dependent $m$ effects into account). Under nominal load condition the PA provides $18.1 \mathrm{dBm}$ output power from a $1.2 \mathrm{~V}$ supply voltage at $1.4 \mathrm{GHz}$. Under load-mismatch, a good agreement between theoretical output power contours and measurements can be seen. The small difference for high VSWRs is due to increased sensitivity of the measurements to parasitics.

Measured efficiency contours are shown in Fig. 4.15(b). Deviation of the measured contours from theoretical contours at the right hand side of the Smith chart is mainly due to the limited loaded quality factor of the output filter (see appendix A) which reduces the efficiency and also due to increased sensitivity of the measurements to parasitics at high VSWRs. However, the proposed theory can (quite) accurately predict the behavior of the class-E PA under load-mismatch.

Measured normalized switch voltage contours are shown in Fig. 4.15(c). The transient waveforms were measured using an N2803A active probe and a $80 \mathrm{GSa} / \mathrm{s}$ Agilent Oscilloscope. The inset at the right upper corner is measured under nominal load condition. Ignoring transient response of the probe, we have $\alpha \approx 0$ and $\beta \approx 0$.

\footnotetext{
${ }^{5}$ Using two cascaded inverters as the driver stage, the input matching is fairly isolated from the output mismatch effects.
} 


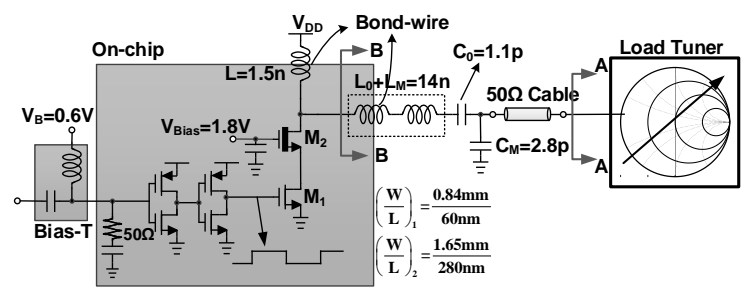

(a)

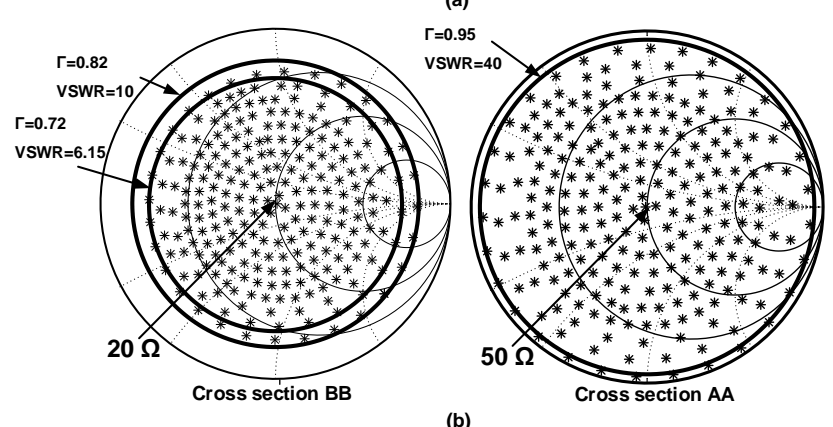

(b)

Figure 4.14: (a) Schematic of the designed PA, the driver stage, matching network and measurement setup. (b) All PA load settings for the measurements at cross sections $\mathrm{AA}$ and $\mathrm{BB}$ at $1.4 \mathrm{GHz}$.

For this nominal load, the maximum normalized switch voltage is (almost) $3.4 V_{D D}$ which results in $\approx 4 \mathrm{~V}$ maximum switch voltage for the $1.2 \mathrm{~V}$ supply voltage. Measured time domain voltage of the switch for four non-nominal loads are also shown in Fig. 4.15(c). In the lower part of the Smith chart $\alpha$ is positive and increases toward lower left corner. In the upper side of the Smith chart $\alpha$ is negative and reduces toward the left hand side; however reverse conduction in the switch limits the negative switch voltage. Because of the limited $Q_{\text {loaded }}$, the measured maximum switch voltage is slightly higher than the theoretical values but the theoretical model still is capable of predicting the behavior of the PA under load-mismatch. To prevent switch degradation (or break down) during measurements, the supply voltage was always tuned to have a maximum switch voltage $4 \mathrm{~V}$ for reliability and (hence) for reproducibility reasons. In post processing the measurements data, the $V_{D D}$ was scaled to the 1.2 nominal value and it was assumed that $P_{\text {out }} \propto V_{D D}^{2}$ which is according to the theory.

Measured average supply/switch current contours are shown in Fig. 4.15(d). A good agreement between the measured contours and the theoretical counterparts can be seen. The chip photo and PCB are shown in Fig. 4.16. 


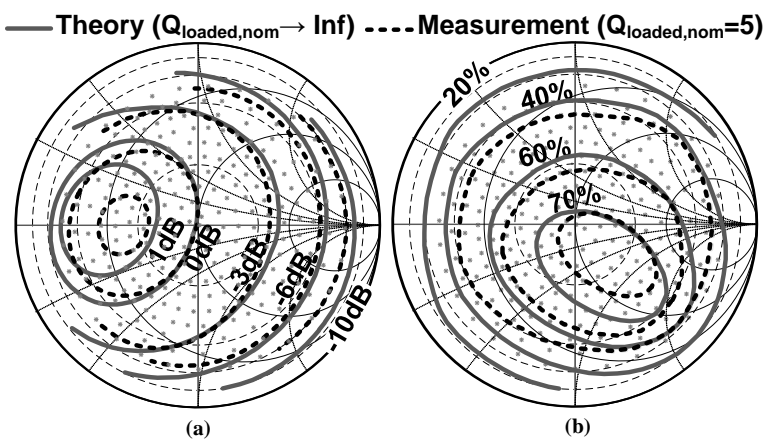

(a)

(b)

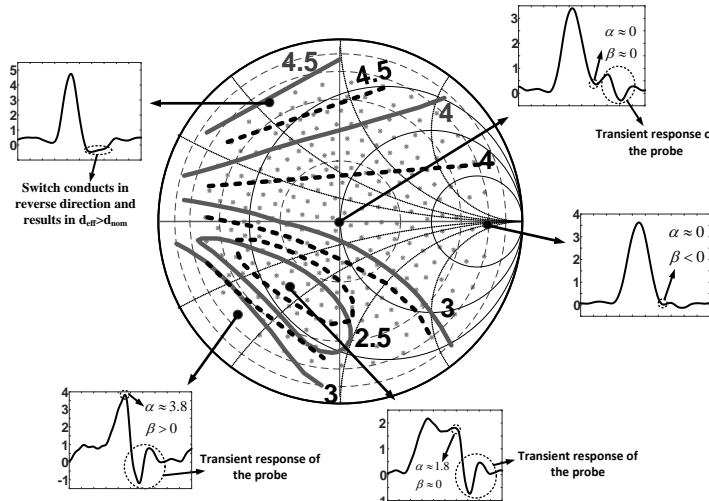

(c)

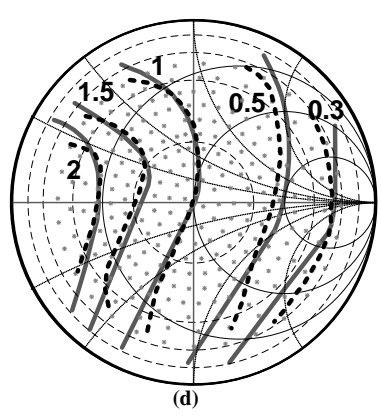

Figure 4.15: Measured load-pull contours at $1.4 \mathrm{GHz}$ (dotted contours) and theoretical contours (solid lines): (a) normalized output power, (b) efficiency, (c) maximum switch voltage normalized to $V_{D D}$ and (d) average switch/supply current normalized to that under nominal condition. 


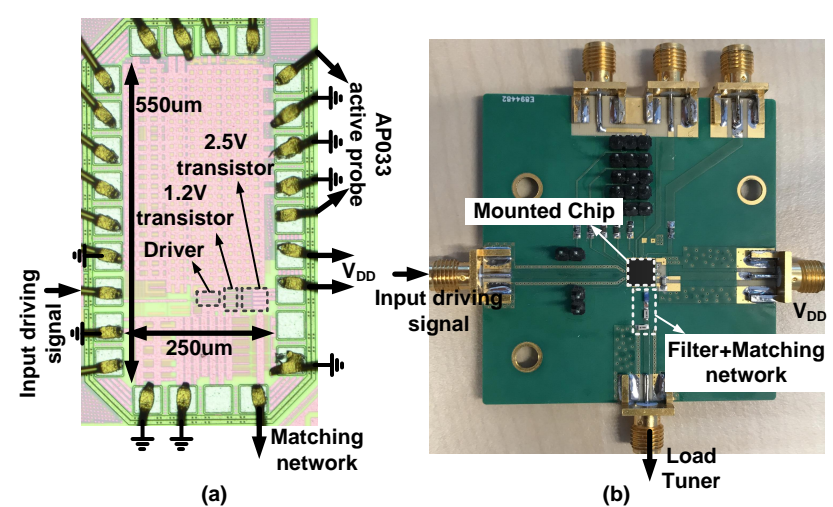

Figure 4.16: (a) Chip microphotograph. (b) Designed PCB setup.

\subsection{Conclusions}

Class-E PAs are typically designed for nominal conditions. However, e.g. antenna impedances are known to vary significantly due to changes in their EM-environment, leading to practical VSWR numbers which are high as 10:1. In this chapter we presented mathematical load-pull analyses for class-E PAs complemented with simulations and measurements results.

It was shown that the performance of the PA as well as the reliability are heavily dependent on load-mismatch. This chapter demonstrates that with load-mismatch, in large areas of the Smith chart the switch voltage and/or the switch current exceeds the nominal value which may cause serious reliability issues; the remaining area then is the class-E PA's safe operating area. Taking into account design margins to handle largerthan-nominal voltages and currents these safe operating areas on the Smith chart increase. Dependencies of the design set and safe operating area on the duty cycle d are also presented, giving e.g. options to rotate and extend the safe operating area on the Smith chart to adapt to the actual load impedances. The theoretical findings were backed-up with circuit simulations including most common non-idealities of class-E PAs as well as load-pull measurements of a class-E PA implemented in $65 \mathrm{~nm}$ CMOS technology at $1.4 \mathrm{GHz}$. 


\section{Bibliography}

[1] A. Ghahremani, A. Annema and B. Nauta, "Load Mismatch Sensitivity of ClassE Power Amplifiers," in IEEE Transactions on Microwave Theory and Techniques, vol. 67, no. 1, pp. 216-230, Jan. 2019.

[2] A. Grebennikov, Switched Mode RF Power Amplifiers. MA, Boston: Newnes Press, 2007.

[3] M. Kazimierczuk, RF Power Amplifiers. Hoboken, NJ: Wiley-IEEE Press, 2008.

[4] S. Cripps, RF Power Amplifiers for Wireless Communications. Norwood, USA: Artech House, 1999.

[5] M. K. Kazimierczuk and D. Czarkowski , Resonant Power Converters. Second edition, New York, USA: Wiley-IEEE Press, 2011.

[6] K. Boyle and M. Leitner, "Mobile phone antenna impedance variations with real users and phantoms," 2011 Int. Antenna Technol. Workshop (iWAT), Hong Kong, 2011, pp. 420-423.

[7] S.M. Bowers, K. Sengupta, K. Dasgupta, B.D. Parker, A. Hajimiri, "Integrated Self-Healing for mm-Wave Power Amplifiers," IEEE Trans. Microw. Theory Techn., vol.61, no.3, pp. 1301-1315, Mar. 2013.

[8] A. Keerti and A. V. H. Pham, "RF characterization of SiGe HBT power amplifiers under load mismatch," IEEE Trans. Microw. Theory Techn., vol. 55, no. 2, pp. 207-214, Feb. 2007.

[9] F.H. Raab, "Effects of circuit variations on the class-E tuned power amplifier," IEEE J. Solid-State Circuits, vol.13, no.2, pp. 239-247, Apr. 1978.

[10] W. H. Cantrell, "Tuning analysis for the high-Q Class-E power amplifier," IEEE Trans. Microw. Theory Techn., vol. 48, no. 12, pp. 2397-2402, Dec. 2000. 
[11] K. Tom and M. Faulkner, "Load pull methodology to characterise class-E outphasing power amplifiers," IET Microw., Antennas and Propag., vol. 6, no. 4, pp. 387-392, Mar. 2012.

[12] R. A. Beltran and F. H. Raab, "Simplified analysis and design of outphasing transmitters using class-E power amplifiers," IEEE PAs for Wireless and Radio Appl. Topical Conf. (PAWR), San Diego, CA, 2015, pp. 1-3.

[13] R. Sadeghpour, A. Nabavi, "Design Procedure of Quasi-Class-E Power Amplifier for Low-Breakdown-Voltage Devices," IEEE Trans. Circuits Syst. I, Reg. Papers, vol.61, no.5, pp. 1416-1428, May 2014.

[14] M. Acar, A. J. Annema and B. Nauta, "Analytical Design Equations for Class-E Power Amplifiers with Finite DC-Feed Inductance and Switch On-Resistance," IEEE Circuits Syst. Int. Symp. (ISCAS), New Orleans, LA, 2007, pp. 2818-2821.

[15] M. Acar, A.J. Annema, B. Nauta, "Variable-Voltage Class-E Power Amplifiers," IEEE/MTT-S Int. Microw. Symp, Honolulu, HI, 2007, pp. 1095-1098.

[16] M. K. Kazimierczuk and K. Puczko, "Class E tuned power amplifier with antiparallel diode or series diode at switch, with any loaded $\mathrm{Q}$ and switch duty cycle," IEEE Trans. Circuits Syst. I, Reg. Papers, vol. 36, no. 9, pp. 1201-1209, Sept. 1989.

[17] M. K. Kazimierczuk and X. T. Bui, "Class E DC/DC converters with an inductive impedance inverter," IEEE Trans. Power Electron, vol. 4, no. 1, pp. 124-135, Jan. 1989.

[18] C. H. Avratoglou and N. Voulgaris, "A new method for the analysis and design of the class E power amplifier taking into account the $Q_{L}$ factor," IEEE Trans. Circuits Syst. I, Reg. Papers, vol. 34, no. 6, pp. 687-691, June 1987.

[19] A. Ghahremani, A. Annema and B. Nauta, "Outphasing Class-E Power Amplifiers: From Theory to Back-Off Efficiency Improvement," IEEE J. Solid-State Circuits, vol. 53, no. 5, pp. 1374-1386, May 2018.

[20] M. Acar, A.J. Annema, B. Nauta, "Analytical Design Equations for Class-E Power Amplifiers," IEEE Trans. Circuits Syst. I, Reg. Papers, vol.54, no.12, pp. 2706-2717, Dec. 2007.

[21] M. Ozen, R. Jos, C.M. Andersson, M. Acar, C. Fager, "High-Efficiency RF Pulsewidth Modulation of Class-E Power Amplifiers," IEEE Trans. Microw. Theory Techn., vol.59, no.11, pp.2931-2942, Nov. 2011. 
[22] T. Quémerais, L. Moquillon, S. Pruvost, J. M. Fournier, P. Benech and N. Corrao, "A CMOS class-A $65 \mathrm{~nm}$ power amplifier for $60 \mathrm{GHz}$ applications," Silicon Monolithic ICs in RF Syst. (SiRF) Topical Meeting, New Orleans, LA, 2010, pp. 120-123.

[23] P. L. Hower and S. Pendharkar, "Short and long-term safe operating area considerations in LDMOS transistors," IEEE Int. Reliability Physics Symp. Proc., 43rd Annu., 2005, pp. 545-550.

[24] I. Aoki, S. Kee, R. Magoon, R. Aparicio, F. Bohn, J. Zachan, G. Hatcher, D. McClymont and A. Hajimiri, "A Fully-Integrated Quad-Band GSM/GPRS CMOS Power Amplifier," IEEE J. Solid-State Circuits, vol.43, no.12, pp.2747,2758, Dec. 2008

[25] E. Rosenbaum, J. C. King and Chenming Hu, "Accelerated testing of $\mathrm{SiO} 2$ reliability," IEEE Trans. Electron Devices, vol. 43, no. 1, pp. 70-80, Jan 1996.

[26] M. Acar, A.J. Annema, B. Nauta, "Generalized Analytical Design Equations for Variable Slope Class-E Power Amplifiers," IEEE 13th Electron. Circuits Syst. (ICECS) Int. Conf., Nice, 2006, pp. 431-434.

[27] T. Mury and V. Fusco, "Exploring figures of merit associated with the suboptimum operation of Class-E power amplifiers," IET Circuits Devices Syst., vol. 1, no. 6, pp. 401-407, Dec. 2007.

[28] T. Suetsugu and M. K. Kazimierczuk, "Design procedure of class-E amplifier for off-nominal operation at 50\% duty ratio," IEEE Trans. Circuits Syst. I, Reg. Papers, vol. 53, no. 7, pp. 1468-1476, July 2006.

[29] T. Suetsugu and M. K. Kazimierczuk, "Off-Nominal Operation of Class-E Amplifier at Any Duty Ratio," IEEE Trans. Circuits Syst. I, Reg. Papers, vol. 54, no. 6, pp. 1389-1397, June 2007.

[30] N. Singhal, N. Nidhi, R. Patel and S. Pamarti, "A Zero-Voltage-Switching Contour-Based Power Amplifier With Minimal Efficiency Degradation Under Back-Off," IEEE Trans. Microw. Theory Techn., vol. 59, no. 6, pp. 1589-1598, June 2011.

[31] N.O. Sokal, A.D. Sokal, "Class E-A new class of high-efficiency tuned singleended switching power amplifiers," IEEE J. Solid-State Circuits, vol.10, no.3, pp. 168-176, June 1975.

[32] F.H. Raab, "Idealized operation of the class-E tuned power amplifier," IEEE Trans. Circuits Syst. I, Reg. Papers, vol.24, no.12, pp. 725-735, Dec. 1977. 
[33] K. Kurokawa, "Power Waves and the Scattering Matrix", IEEE Trans. Microw. Theory Techn., vol. 13, no. 2, pp. 194-202, Mar. 1965.

[34] B. Razavi, Design of Analog CMOS Integrated Circuits. London, USA: McGrawHill, 2001.

[35] M. Schenkel, P. Pfaffli, S. Mettler, "Measurements and 3D Simulations of FullChip Potential Distribution at Parasitic Substrate Current Injection," 30th SolidState Device Research Conf. Proc., pp. 600-603, Sept. 2000.

[36] A. J. Annema, G. J. G. M. Geelen and P. C. de Jong, "5.5-V I/O in a 2.5-V 0.25-um CMOS technology," IEEE J. Solid-State Circuits, vol. 36, no. 3, pp. 528-538, Mar. 2001.

[37] A. Mazzanti, L. Larcher, R. Brama and F. Svelto, "Analysis of reliability and power efficiency in cascode class-E PAs," IEEE J. Solid-State Circuits, vol. 41, no. 5, pp. 1222-1229, May 2006.

[38] M. P. van der Heijden and M. Acar, "A radio-frequency reconfigurable CMOSGaN class-E Chireix power amplifier," 2014 IEEE MTT-S Int. Microw. Symp. (IMS2014), Tampa, FL, 2014, pp. 1-4. 


\section{Chapter 5}

\section{Outphasing Class-E PAs: From Theory to Back-off Efficiency Improvement}

\subsection{Introduction}

Nowadays, modern communication systems necessitate complex modulated signals (e.g. OFDM and 64QAM) with high peak to average power ratios (PAPRs) ${ }^{1}$. The demands on the power amplifier (PA) in these systems are manifold: the PA mainly works in back-off, must be sufficiently robust and must have sufficiently high efficiency.

Among the various classes of PAs, class-E PAs are of great interest. Zero Voltage Switching (ZVS) and Zero Slope Switching (ZSS) conditions for the switch waveform in class-E PAs result in non-overlapping voltages and currents for the switch and hence yield high efficiency (ideally 100\%) [2]. Due to switch-mode operation, a single class-E with constant supply voltage only allows phase modulation or On-Off Keying (OOK) modulation. Supply modulation through Envelope Elimination and Restoration (EER) [3] or load modulation through outphasing [4] is necessary to also enable amplitude modulation.

Outphasing class-E PAs (OEPAs) are getting more popular. Their performance is weakly dependent on process variations due to the switch-mode operation of the branch amplifiers [5]. Other advantages of OEPAs include maintaining high efficiency at a relatively wide output power back-off range and digital compatibility due to the phase-only control [6].

\footnotetext{
${ }^{1}$ This chapter consists of material previously published in IEEE Journal of Solid-States (JSSC) [1] which was re-structured for readability in thesis form.
} 
However, some issues with OEPAs need to be addressed. Firstly, the maximum voltage of the switches can be very high $[7,8]$. Being implemented by transistors, there is a trade off between switch performance (e.g. on-resistance, drive power, speed) and reliability (e.g. life time) which makes the design of class-E PAs challenging [9]. Secondly, high order modulated signals require a high Output Power Dynamic Range (OPDR). However, mismatch between the two paths in outphasing system limits the OPDR. Thirdly, OPEAs require power combiners. For high efficiency at power backoff non-isolating power combiners are used [10]. This results in load-pulling between the two branch amplifiers yielding the ZVS and ZSS conditions to be violated and consequently dropping the efficiency well below it's maximum (ideal) 100\% [11].

Recently some papers reported on improving the efficiency of OEPAs in power back-off. In [11], variable duty cycle combined with variable drain capacitance and with a tunable load network were used to maintain ZVS and ZSS conditions in power back-off up to (ideally) $9 \mathrm{~dB}$ back-off. The CMOS implementation of the technique, presented in [11], was employed in [12] at $1.85 \mathrm{GHz}$. For higher than (ideally) $9 \mathrm{~dB}$ back-off levels, the outphasing technique employed in $[11,12]$ resulted in a reduction of the efficiency due to non-zero switching losses (non-ZVS condition). Due to practical limitations on the minimum feasible duty cycle at high frequencies in [12], the ZVS and ZSS conditions were satisfied for $6 \mathrm{~dB}$ of back-off range which yielding lower than $10 \%$ Power Added Efficiency (PAE) at 10dB back-off. Furthermore, amplitude errors limited the OPDR to $30 \mathrm{~dB}$ in [11]. In [13] an isolating power combiner was used and supply voltage switching was implemented to maintain high efficiency at power backoff. However, this latter technique requires a highly efficient DC-DC converter not to compromise the overall efficiency of the OEPAs. Non-isolating package-integrated transformer based power combiners with Chireix compensation elements along with cleverly chosen class-E PA design parameters (denoted as load-insensitive class-E PA) was introduced in [6]. This load-insensitive class-E design concept provides $100 \%$ efficiency only for ohmic loads while Chireix compensation elements null the imaginary parts of the branch PA loads at (typically) only two power back-off levels. However, for higher back-off levels, OEPA efficiency reduces rapidly due to the non-zero imaginary part of the loads. Adaptive compensation elements [14] or 4-way outphasing systems [15] can be used to reduce the imaginary part of the loads at lower power levels but add complexity and lossy elements at the output that compromise the efficiency. Variable duty cycle was used in [16] to ensure load-insensitive design conditions across a wide frequency range. The technique in [16] aimed at minimizing the back-off efficiency drop in the frequency range $1.84-2.14 \mathrm{GHz}$ with respect to the nominal design at $2.14 \mathrm{GHz}$.

In [8], we presented a new technique to improve the back-off efficiency for OEPAs with load-insensitive class-E designs. Output power contours and efficiency contours of the class-E PAs on the Smith chart were shown to rotate and the power contours 
were shown to shift by changing just a few class-E design parameters. This rotation and shift were used to considerably improve the efficiency of OEPAs at back-off. Moreover, it was shown that this technique also improves the OPDR and reduces switch voltage stress at back-off.

In contrast to $[11,12]$, in [8] we used a load-insensitive design which ideally yields $100 \%$ efficiency at (almost) 0dB and 10dB back-off levels and more than $95 \%$ efficiency between $0 \mathrm{~dB}$ and $12 \mathrm{~dB}$ back-off levels. Moreover, theoretically this yields an infinite OPDR without requiring to tune any parameter. Using our technique in [8], the second compensation point can be shifted up to $20 \mathrm{~dB}$ into back-off. This not only can improve the average efficiency of the OEPAs for modulated signals with high PAPRs, but is also promising for applications with output power control or for multimode (standard) PAs with different average output power levels [17-19]. Also, our technique does not require to tune the load while parameter tuning is done prior to the series output filter of the class-E PAs to change the (very non-sinusoidal) voltage at the switching node. Moreover, parameter tuning is employed at power back-off which helps to obtain high efficiency both at back-off and at maximum output power. This chapter provides more insight to the technique, shows detailed design considerations and provides more measurement results at a higher frequency.

There are other previously published works on the derivation of class-E design equations as well as the effect of changing design parameters on performance and reliability of class-E PAs. The design equations for variable duty cycle, ZVS and ZSS conditions were presented in [20] while [21] derives similar design equations for variable duty cycle and (only) ZVS condition. However, under non-nominal load conditions ZVS and ZSS conditions, both are violated. As a result, the design equations in [20] and [21] cannot be employed to load-pulling nor to study the effect of changing design parameters on load-pull contours. Therefore, new mathematical design equations were derived for general switching conditions (non-ZVS and non-ZSS) similar to [7,22] but now including the DC-feed inductor loss as well as the switch conduction loss. These derivations were included in chapter 4 and in this current chapter only the results are summarized and used to explain our efficiency enhancement technique.

A few publications provide a theoretical model for OEPAs. The presented analyses in $[23,24]$ are (only) for a special case; using ideal loss-less components, RF choke as the DC-feed inductor and $50 \%$ duty cycle. The presented semi-analytical design methodology in [25] is for a more general case of arbitrary DC-feed inductor and arbitrary duty cycle. However, the presented theory only works for one outphasing angle where both the branch class-E PAs satisfy a predefined switching conditions (e.g. the ZVS). Then, to have a full picture of the outphasing operation, in [25], simulations were conducted using a commercial computer program.

The chapter is organized as follows. A review of the class-E PAs, a load-pull study of load-insensitive class-E PAs and the effect of changing class-E design parameters on 
load-pull contours is presented in section 5.2. The presented approach in the current chapter to study the OEPAs (in section 5.3) and our efficiency improvement technique at back-off (in section 5.4) is based on the load-pull study of the class-E PAs presented in chapter 4 which are also briefly reviewed in section 5.2. This approach makes it possible to theoretically obtain the PA loads, normalized output power, efficiency and reliability related maximum switch voltage for all the outphasing angles and as a result the OPDR. The presented approach can be used with any arbitrary DC-feed inductor (including loss), arbitrary duty cycle, considering the switch conduction loss and we use standard Smith chart representation. Second order effects that come into play for hardware realizations are discussed in section 5.5. The design of a demonstrator OEPA in $65 \mathrm{~nm}$ CMOS that implements the proposed technique and measurement results thereof are given in sections 5.6 and 5.7, respectively. Finally, the conclusions are summarized in section 5.8.

\subsection{Class-E Power Amplifier Basics}

Fig. 5.1a shows a class-E PA, where the MOS transistor acts as a switch driven by a square wave input signal with (angular) frequency $\omega_{0}$ and duty cycle scaling factor of $d$ where $d=1$ corresponds to $50 \%$ duty cycle. The general switching conditions are defined as

$$
v_{c}\left(\frac{2 \pi}{\omega_{0}}\right)=\alpha V_{D D} \quad \text { and } \quad \frac{d v_{c}}{d t}\left(\frac{2 \pi}{\omega_{0}}\right)=\beta \omega_{0} V_{D D}
$$

where $v_{c}$ and $V_{D D}$ are the switch and supply voltage, respectively. $L$ and $C$ form the primary LC-tank to shape the switch voltage according to the required values of $\alpha$ and $\beta$, shown in Fig. 5.1b, [26,27]. The relative resonance frequency of this tank is defined [22] as

$$
q=\frac{1}{\omega_{0} \sqrt{L C}}
$$

The second tank, by $L_{0}$ and $C_{0}$, is a band pass filter to filter out load current harmonics. A matching network (not shown here for simplicity) provides the load of the PA $(R+j X)$ from the nominal $50 \Omega$ antenna impedance. The relation between circuit elements $(L, C, X$ and $R), V_{D D}, \omega_{0}$ and output power $P_{\text {out }}$ are formulated by a K-design set [22]:

$$
K=\left\{K_{L}, K_{C}, K_{X}, K_{P}\right\}=\left\{\frac{L \omega_{0}}{R}, R C \omega_{0}, \frac{X}{R}, \frac{R P_{\text {out }}}{V_{D D}^{2}}\right\}
$$

The K-design set for non-ZVS, non-ZSS, arbitrary $d$ and $q$ and taking into account the switch resistance $R_{o n}$ is derived in [7], yielding $K=K(q, d, m, \alpha, \beta)$, where $m=$ $\omega_{0} R_{o n} C$. 


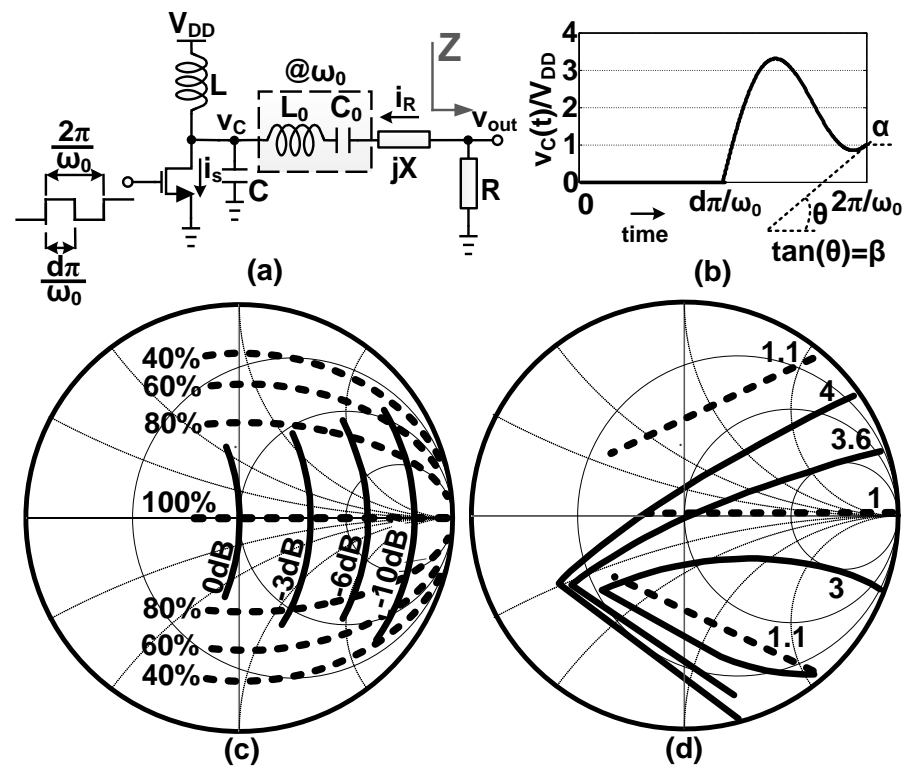

Figure 5.1: (a) Single-ended Class-E PA, (b) normalized switch voltage. Loadpull plots for $q=1.3, d=1$ and $m=0$, (c) normalized output power (solid) and efficiency (dotted) contours, (d) maximum switch voltage normalized to $V_{D D}$ (solid) and normalized output voltage amplitude (dotted).

\subsubsection{Load-pulling class-E PAs}

For the so-called load-insensitive design $[6,28]$, a class-E PA is conventionally designed to have $q=1.3, d=1$, ZVS $(\alpha=0)$ and ZSS $(\beta=0)$. Assuming an ideal switch, $(m=$ $0)$, the K-design set elements can be obtained from e.g. [7] as $\left\{K_{L}, K_{C}, K_{X}, K_{P}\right\}=$ $\{1.04,0.58,0.28,1.26\}$. For given $P_{\text {out }}, \omega_{0}$ and $V_{D D}$, the component values in Fig. 5.1a can then be calculated from the K-design set equations in (5.3).

For a load-pulling analysis, this ideal class-E PA is subjected to different loads. For this, $j X$ is kept constant and only the load impedance $Z$, represented by its nominal (real) value $R$ in Fig. 5.1a, is changed. For simplicity we normalize both the real and imaginary part of $Z$ to $R$ :

$$
Z=k R+j k^{\prime} R
$$

Note that for the nominal load, $k=1$ and $k^{\prime}=0$. For fixed $q, d$ and $m$, under nonnominal load conditions ZVS and/or ZSS conditions are violated. A full mathematical derivation of the switch voltage and current is beyond the scope of this chapter and can be found in e.g. [7].

These equations can be rewritten to get important properties of both the switch voltage $v_{C}(t)$ and the switch current $i_{s}(t)$. This allows to derive e.g. $P_{\text {out }}$ normalized 
to that at nominal load conditions, the efficiency, the maximum switch voltage $V_{c, M a x}$. normalized to $V_{D D}$ and the output voltage amplitude $V_{\text {out }}$ normalized to that at nominal load conditions. All these can be derived as a function of $k$ and $k^{\prime}$ as defined in (5.4), independent from $P_{\text {out }}$ at nominal conditions, the frequency and the nominal load value $R$. As a result, for any set of $q, d$ and $m$, we can now plot contours on a Smith chart with $R$ as the reference impedance, showing the impact of load changes on the performance and behavior of class-E PAs.

For example for a load-insensitive class-E PA with an ideal switch $(m=0)$, the load-pull contours are shown in Fig. 5.1c and d for a part of Smith chart. The normalized output power and the efficiency contours are shown in Fig. 5.1c with solid and dotted lines, respectively. Fig. 5.1c shows that for real loads (for impedances $\operatorname{Re}\{Z\} \geq R$ ) the efficiency is (ideally) $100 \%$ while the output power can be lowered. A non-zero imaginary part of the load will result in $\alpha \neq 0$ at switching moment and hence causes switching loss (because of discharging the non-zero capacitor voltage) which reduces the efficiency. $V_{c, \text { Max }}$. contours normalized to $V_{D D}$ are shown in Fig. 5.1d. For real loads $\left(\operatorname{Re}\{Z\} \geq R\right.$ ) the $V_{c, \text { Max }}$. stays close to (but is lower than) that for the nominal load. Toward the upper side of the Smith chart the $V_{c, \text { Max. }}$ increases. Normalized output voltage amplitude across the load, shown in Fig. 5.1d, shows a symmetrical behavior with respect to real axis. These load-pull contours will be used in the next section to describe the behavior of OEPAs and to introduce our method to increase power efficiency in back-off.

\subsubsection{Effect of changing $q$ and $d$ on the load-pull contours}

Parameters $q$ and $d$ have a major impact on the load-pull contours shown in Fig. 5.1. In this section we assume that the class-E PA is initially designed for $q=1.3$ and $d=1$. Then we change the parameter $q$ (by e.g. changing the capacitor $\mathrm{C}$ ) and/or change the parameter $d$ and plot the resulting load-pull contours. Again, these contours are independent from $P_{\text {out }}, \omega_{0}$ and the nominal load $R$.

The load-pull plots are shown in Fig. 5.2 for changing $q$ from 1.3 to 1.2, respectively 1.4. The shape of the normalized output power, efficiency and $V_{c, \text { Max }}$. contours hardly change except for a rotation: there is clockwise (anti-clockwise) rotation for higher (lower) $q$. The normalized output voltage contours, shown in Fig. 5.2b and d, are rotated and changed in shape. Similar contours can be derived and plotted to show the effect of changing $d$; then clockwise (anti-clockwise) rotation occurs for lower (higher) $d$. These plots are shown in Appendix B. 

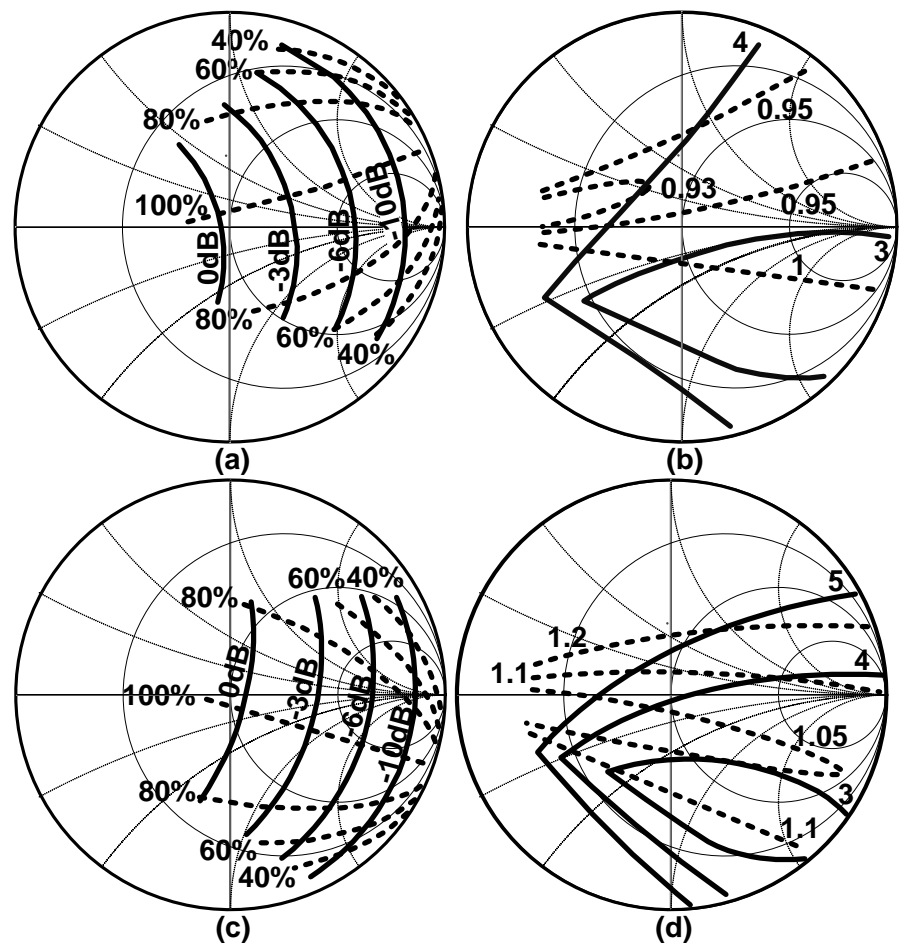

(d)

Figure 5.2: Effect of changing q from 1.3 to $1.2(\mathrm{a}, \mathrm{b})$, respectively $1.4(\mathrm{c}, \mathrm{d})$ on the load-pull plots for $d=1$ and $m=0$. (a,c) Normalized output power (solid) and efficiency (dotted) contours. (c,d) $V_{c, M a x}$. normalized to $V_{D D}$ (solid) and normalized output voltage amplitude (dotted).

\subsection{Simplified Theory of OEPAs}

An outphasing class-E PA with a signal component separator (SCS), two class-E branch PAs and a combiner is shown in Fig. 5.3a. The SCS generates two driving waveforms with phase difference of $\Delta \theta_{i n}$ according to the input signal amplitude.

To be able to map the results of section 5.2, we assume that both class-E PAs, conventionally, are designed to have $q=1.3, d=1$ and $\alpha=\beta=0$ for a load $Z_{1}=Z_{2}=R$. The combiner at the output sums the output voltages to reconstruct an amplified replica of the input signal. Two compensating elements, $\pm j B_{c}$ are used to compensate the imaginary part of the loads at two specific outphasing angles. In this thesis a transmission-line based combiner is used.

Let's consider the part in the dotted box in Fig. 5.3a and assume voltages at the branch PAs' outputs as

$$
v_{\text {out }_{1,2}}(t)=V_{\text {out }_{1,2}} \sin \left(\omega_{0} t+\phi_{v_{1,2}}\right)
$$




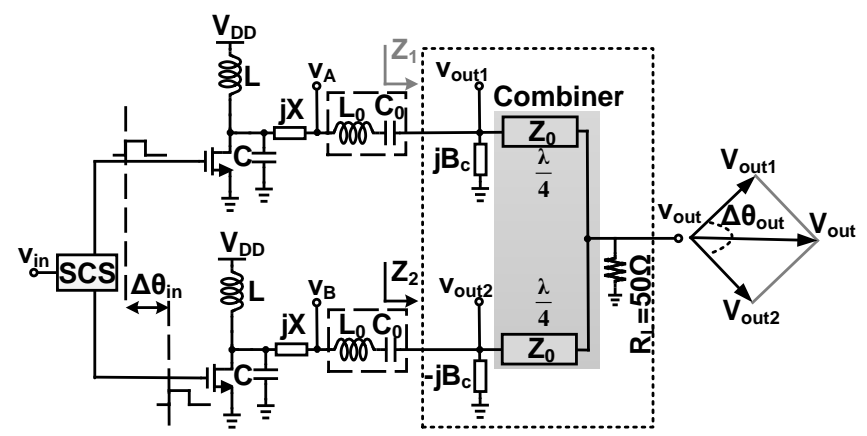

(a)

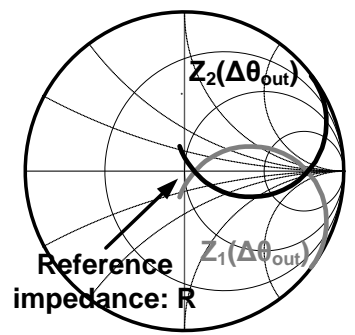

(b)

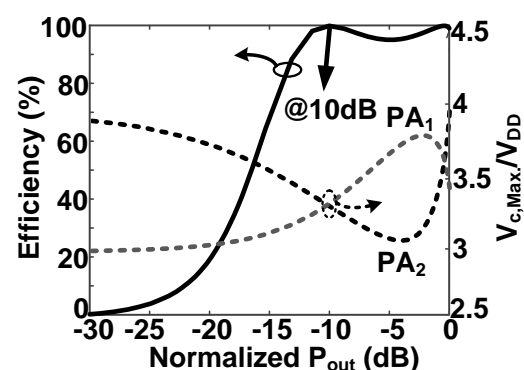

(c)

Figure 5.3: (a) Outphasing class-E PA with transmission-line based combiner. (b) $Z_{1,2}$ for $0<\Delta \theta_{\text {out }}<\pi$ and compensation at outphasing angles $\frac{\pi}{5}$ and $\pi-\frac{\pi}{5}$. (c) Efficiency vs normalized output power (solid) and $V_{c, \text { Max }}$. normalized to $V_{D D}$ for PA1 (dotted-grey) and PA2 (dotted-dark).

where $V_{\text {out }_{1,2}}$ and $\phi_{v_{1,2}}$ are the amplitudes and initial phases with respect to a reference time, respectively. The circuit is linear and can be solved in phasor domain which leads for the apparent load impedances of the two branch PAs to:

$$
\begin{aligned}
& \frac{1}{Z_{1}}=+j B_{c}+\frac{R_{L}}{Z_{0}^{2}}\left(1+\frac{V_{\text {out }, 2}}{V_{\text {out }, 1}} e^{-j \Delta \theta_{\text {out }}}\right) \\
& \frac{1}{Z_{2}}=-j B_{c}+\frac{R_{L}}{Z_{0}^{2}}\left(1+\frac{V_{\text {out }, 1}}{V_{\text {out }, 2}} e^{+j \Delta \theta_{\text {out }}}\right)
\end{aligned}
$$

where $\Delta \theta_{\text {out }}=\phi v_{1}-\phi v_{2}$ is denoted as the outphasing angle. To simplify the analysis, let's assume $\frac{V_{\text {out } 1}}{V_{\text {out } 2}}=1$. We will show the validity of this assumption later in this section. The $\Delta \theta_{\text {out }}$-dependent impedance seen by each PA, $Z_{1,2}$, then is

$$
\begin{gathered}
\frac{1}{Z_{1}}=\frac{2 R_{L}}{Z_{0}^{2}} \cos ^{2}\left(\frac{\Delta \theta_{\text {out }}}{2}\right)+j\left(-\frac{R_{L}}{Z_{0}^{2}} \sin \left(\Delta \theta_{\text {out }}\right)+B_{c}\right) \\
\frac{1}{Z_{2}}=\frac{2 R_{L}}{Z_{0}^{2}} \cos ^{2}\left(\frac{\Delta \theta_{\text {out }}}{2}\right)-j\left(-\frac{R_{L}}{Z_{0}^{2}} \sin \left(\Delta \theta_{\text {out }}\right)+B_{c}\right)
\end{gathered}
$$


where we assume $Z_{0}=\sqrt{2 R R_{L}}$. For $B_{c}<\frac{R_{L}}{Z_{0}^{2}}$, there are two outphasing angles for which the imaginary part of the loads $Z_{1,2}$ is zero. $Z_{1,2}$ for a range $0<\Delta \theta_{\text {out }}<\pi$ are shown in Fig. 5.3b on the Smith chart for $B_{c}=\frac{1}{2 R} \sin (\pi / 5)$, assuming $R$ as the reference impedance.

Having PA loads $Z_{1,2}$, shown in Fig. 5.3b, on top of the load-pull contours of the normalized output voltage amplitude shown in Fig. 5.1d, yields two important observations. Firstly, the output voltage of the branch PAs is not constant across different outphasing angles and therefore, the PAs cannot be modeled as ideal voltage sources as was done in e.g. [6] (although the same final results for $Z_{1,2}$ were obtained). Secondly, because of the symmetry of the PA loads $Z_{1,2}$ and of the normalized output voltages contours with respect to the real axis, for all outphasing angles $\frac{V_{\text {out }, 1}}{V_{\text {out }, 2}}=1$ which proves the validity of our assumption leading to (5.7).

To get the output power and the efficiency for an OEPA as a function of power back-off, the normalized power contours and the efficiency contours of Fig. 5.1c can be combined with the $\Delta \theta_{\text {out }}$-dependent load impedance for the two branch PAs of an OEPA, described in (5.7) and shown in Fig. 5.3b. Then for each $\Delta \theta_{\text {out }}$, the output power and the efficiency of the branch class-E PAs follow. Due to symmetrical PA loads and contours with respect to the real axis, the output power and efficiency of both branch PAs are identical.

Combining the output power as a function of $\Delta \theta_{\text {out }}$ and the efficiency as a function of $\Delta \theta_{\text {out }}$ yields Fig. $5.3 \mathrm{c}$, that shows the efficiency of the OEPA plotted versus the (normalized) output power. The second compensation point is located at $10 \mathrm{~dB}$ backoff where the efficiency is (ideally) $100 \%$. Similarly, the $V_{c, \text { Max }}$. (normalized to $V_{D D}$ ) for the two branch PAs can also be derived as a function of the power back-off. Fig. $5.3 \mathrm{c}$ shows that the $V_{c, \text { Max }}$. for PA2 increases at back-off. We will also address this issue in the measurement section. All plots of Fig. 5.3c are valid under the assumption of an ideal OEPA with an ideal combiner and assuming a very high loaded quality factor for the $L_{0}-C_{0}$ filter.

\subsubsection{Output Vectors' Amplitude Mismatch}

In theory, the branch class-E PAs are identical and hence have $0 \%$ mismatch/error between the output voltage amplitudes $V_{\text {out }, 1,2}$. The normalized output power, shown in Fig. $5.3 \mathrm{c}$, is then 0 for $\Delta \theta_{\text {out }}=\pi$ which yields an infinite OPDR.

Any mismatch between the two paths in an outphasing system that causes amplitude errors between the two output vectors $V_{\text {out } 1}$ and $V_{\text {out } 2}$, reduces the OPDR. Using the vector diagram in Fig. 5.3a and assuming a relative amplitude error $\epsilon$ between the two vectors, $V_{\text {out } 1}=V$ and $V_{\text {out } 2}=V(1+\epsilon)$, the maximum attainable OPDR 
can then be written as

$$
O P D R(d B)=20 \log \left(\frac{\max \left(V_{\text {out }}\right)}{\min \left(V_{\text {out }}\right)}\right)=20 \log \left(1+\frac{2}{\epsilon}\right)
$$

Using (5.8), to have an OPDR better than $60 \mathrm{~dB}$ [28], the amplitude error should be kept below $0.2 \%$ while mismatch less than $6.5 \%$ keeps the OPDR better than $30 \mathrm{~dB}[11]$.

One of the mechanisms that leads to amplitude error, is the residual impedance of the series filter $L_{0}-C_{0}$ due to e.g. frequency deviations or components spread. For instance, the residual impedance at $\Delta \omega$ deviation from the center frequency $\omega_{0}=$ $\frac{1}{\sqrt{L_{0} C_{0}}}$ can be written as

$$
Z_{r}(\Delta \omega)=L_{0}\left(\omega_{0}+\Delta \omega\right) j-\frac{j}{C_{0}\left(\omega_{0}+\Delta \omega\right)} \approx 2 L_{0} \Delta \omega j
$$

For $\Delta \omega=0, Z_{r}=0$ and the filter passes the first harmonic of the signal without any error. However, non-zero $Z_{r}$ causes a voltage division at the inputs of the combiner and hence creates error.

To find a simple quantitative model, consider the OEPA system, shown in Fig. $5.3 \mathrm{a}$ and assume that, for small $\Delta \omega$, the combiner, the $q$ parameters, the compensation elements impedance and the first harmonic of the signals at nodes A and B (before the filter) are not affected. Then we can assume identical first harmonics $V_{A}=V_{B}=V$ for nodes $\mathrm{A}$ and $\mathrm{B}$ for identical class-E branch PAs. For minimum output power, $\Delta \theta_{\text {out }}=\pi$, and therefore $V_{\text {out } 1}$ and $V_{\text {out } 2}$ can be written as

$$
\begin{aligned}
& V_{\text {out } 1}\left(\Delta \theta_{\text {out }}=\pi\right)=\frac{Z_{1} V_{A}}{Z_{1}+Z_{r}}=\frac{V}{1-2 L_{0} B_{c} \Delta \omega} \\
& V_{\text {out } 2}\left(\Delta \theta_{\text {out }}=\pi\right)=\frac{Z_{2} V_{B}}{Z_{2}+Z_{r}}=\frac{V}{1+2 L_{0} B_{c} \Delta \omega}
\end{aligned}
$$

where we replaced $Z_{1}$ and $Z_{2}$ from (5.7) for $\Delta \theta_{\text {out }}=\pi$ and $Z_{r}$ from (5.9). Assuming the loaded $\mathrm{Q}$ of the filter as $Q_{L}=\frac{L_{0} \omega_{0}}{R}$ and locating the second compensation point at $10 \mathrm{~dB}$ back-off $\left(B_{c}=\sin (\pi / 5) / 2 R\right)$,

$$
\frac{V_{\text {out } 1}}{V_{\text {out } 2}}\left(\Delta \theta_{\text {out }}=\pi\right)=\frac{1+Q_{L} \sin \left(\frac{\pi}{5}\right) \frac{\Delta \omega}{\omega_{0}}}{1-Q_{L} \sin \left(\frac{\pi}{5}\right) \frac{\Delta \omega}{\omega_{0}}}
$$

$Q_{L}=5, \frac{\Delta \omega}{\omega_{0}}=+1 \%$ and $\frac{\Delta \omega}{\omega_{0}}=+5 \%$ yield $\frac{V_{\text {out } 1}}{V_{\text {out } 2}}$ equal to 1.06 and 1.34 , respectively. According to (5.8), and assuming that $Z_{r}$ will not affect maximum $P_{\text {out }}$, these amplitude errors limit OPDR to $30 \mathrm{~dB}$ respectively $16 \mathrm{~dB}$. Moreover, $\frac{\Delta \omega}{\omega_{0}}=-1 \%$ and $\frac{\Delta \omega}{\omega_{0}}=-5 \%$ results in $\frac{V_{\text {out } 1}}{V_{\text {out }}}$ equal to 0.94 and 0.74 , respectively, which limits OPDR to $31 \mathrm{~dB}$ and $19 \mathrm{~dB}$, respectively. 


\subsection{Back-off efficiency improvement technique}

This section presents detailed discussions of the technique presented in [8] to improve the back-off efficiency of the OEPAs. The starting point is again a conventional OEPA with $q_{1}=q_{2}=1.3$ and with the second compensation points located at $10 \mathrm{~dB}$ back-off.

\subsubsection{Rotation}

Adaptively changing the compensating elements $\pm j B$ in the OEPA or employing 4way outphasing system can improve the efficiency at more back-off levels. However, a more efficient way - both for efficiency and for integration - is to change the parameter $q$ of both branch PAs. Fig. 5.2 shows that by increasing (decreasing) the parameter $q$, the power and efficiency contours rotate in the clockwise (anti-clockwise) direction.

Using Fig. 5.3b, to shift the compensation point deeper into back-off, the contours for PA1 should rotate in the clockwise direction and simultaneously the contours for PA2 must rotate in the anti-clockwise direction. To accomplish this, for PA1 the $q$ must be increased (e.g. from 1.3 to 1.4) and for PA2 it must be reduced (e.g. from 1.3 to 1.2$)$.

To find the efficiency of the OEPA having different $q$ for the two branch PAs, again the PA loads $Z_{1,2}$ need to be known. However, whereas in the previous section $V_{\text {out } 2}=V_{\text {out } 1}$ due to having the same $q$ in both branch PAs, this condition is inherently violated now, see e.g. Fig. 5.2b and d. Therefore, (5.7) cannot be used and the general equation (5.6) must be employed. For each outphasing angle $\Delta \theta_{\text {out }}$, we use a simple iterative method to find the PA loads; in it we start at $V_{\text {out } 1}=V_{\text {out } 2}$ and estimate the loads from (5.6). For the calculated loads, we use the data in Fig. 5.2b and d to update the estimated $\frac{V_{\text {out } 1}}{V_{\text {out } 2}}$ and to then recalculate the loads from (5.6). We terminated this iterative routine upon reaching a sufficiently low change in $\frac{V_{\text {out } 1}}{V_{\text {out } 2}}$; typically lower than $1 \%$ error was reached within 2 or 3 iterations. The PA loads $Z_{1,2}$ and $\frac{V_{\text {out } 1}}{V_{\text {out } 2}}$ for $q_{1}=1.4$ and $q_{2}=1.2$ are shown with dashed lines for $0 \leq \Delta \theta_{\text {out }} \leq \pi$ in Fig. 5.4a and b. For comparison, the corresponding curves for a conventional OEPA $\left(q_{1}=q_{2}=1.3\right)$ are shown using solid curves.

After obtaining the PA loads $Z_{1,2}$ as a function of $\Delta \theta_{\text {out }}$, for specific $q_{1}$ and $q_{2}$, and using the load-pull contours in Fig. 5.2, the output power, efficiency and the normalized $V_{c, \text { Max }}$. can directly be obtained. These are shown in Fig. $5.4 \mathrm{c}$ and $\mathrm{d}$ with dashed lines.

Fig. 5.4c shows that the second compensation point is shifted from the initial $10 \mathrm{~dB}$ back-off (for $q_{1}=q_{2}=1.3$ ) to almost $17 \mathrm{~dB}$ into back-off for $q_{1}=1.4$ and $q_{2}=1.2$; here the power efficiency is $100 \%$, see Fig. 5.4c. Fig. 5.4a shows the PA loads $Z_{1,2}$; the negative real part of $Z_{2}$ for $\Delta \theta_{\text {out }}$ close to $\pi$ implies that PA2 absorbs part of the 
power provided by PA1. The extension of the load-pull contours, presented in Fig. 5.1c and $\mathrm{d}$ for positive loads, toward negative impedances is presented in Appendix C. Note that for the OEPA shown in Fig. 5.3a, there is no risk for the stability as the loop gain, for the loop consists of the nodes $V_{D D}-v_{A}-v_{\text {out }, 1}-v_{\text {out }}-v_{\text {out }, 2}-v_{B}-V_{D D}$, is always less than unity due to the loss of the components, switch loss and mainly due to the fact that $P_{\text {out }}>=0$.

The (almost) $10 \%$ unbalance between the two branch PAs, visible in Fig. 5.4b for $q_{1}=1.4$ and $q_{2}=1.2$, limits the OPDR according to (5.8) to about $27 \mathrm{~dB}$. Shifting the second compensation point more into back-off with extra rotation will limit the OPDR more. The unbalance due to rotation, however, can be used to compensate the amplitude error due to e.g. frequency deviations, discussed in section 5.3.1. For negative frequency deviations where $\frac{V_{\text {out } 1}}{V_{\text {out } 2}}$ goes below 1 , the rotation according to Fig. 5.4 can be employed to increase the amplitude ratio toward unity and to improve the OPDR. For positive frequency deviations, where $\frac{V_{\text {out } 1}}{V_{\text {out } 2}}$ increases above unity, rotation in reverse direction by reducing $q_{1}$ or/and increasing $q_{2}$ should be employed to reduce the amplitude ratio. In this case, however, the back-off efficiency will reduce compared to the conventional design having $q_{1}=q_{2}=1.3$. The next section extends the shift technique with the rotation to compensate for this efficiency drop.

Fig. 5.4d shows the $V_{c, \text { Max }}$. versus the normalized output power (back-off), illustrating that the $V_{c, \text { Max }}$. in back-off can be reduced with shifting the second compensation point further into back-off.

\subsubsection{Shift}

Reducing the parameters $d$ and $q$ for both branch PAs results in rotation in the opposite directions. Therefore, for a lower $d$ (e.g. 0.7), a lower $q$ (e.g. 1.1) can be found to have almost same efficiency contours for the branch PAs at a lower output power level. This corresponds to a shift of power contours to the left on Smith chart for almost the same efficiency contours. As a result the output power at (e.g.) the second compensation point can be lowered while the efficiency is $100 \%$ : the second compensation can be shifted to a lower output power levels.

This technique can be combined with the rotation technique to shift the second compensation point more into back-off. For this, the $d$ for both branch PAs are reduced to 0.7 for $q_{1}=q_{2}=1.1$ to shift the power contours. Subsequently $q_{1}$ is increased to 1.25 and $q_{2}$ is reduced to 1.02 to rotate both the power and the efficiency contours. Following the same procedure as in the previous section, the load-pull contours can be plotted and a simple iterative procedure can be used to find $\frac{V_{\text {out } 1}}{V_{\text {out }}}$ for each $\Delta \theta_{\text {out }}$ which results in the PA loads $Z_{1,2}$. For length reasons the load-pull contours are not shown here but the effect of the shift-rotation technique on the OEPA performance and behavior is shown in Fig. 5.4 with dotted lines. Fig. 5.4b shows 


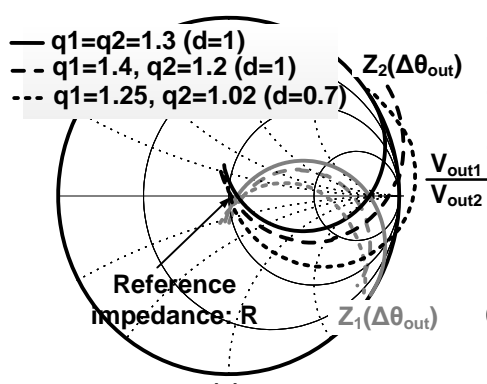

(a)

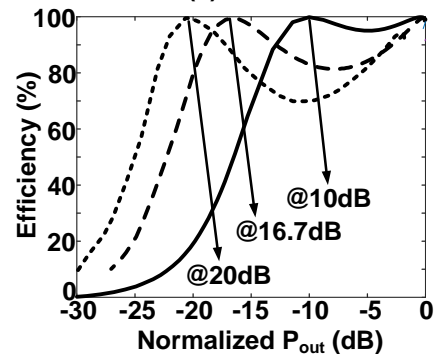

(c)

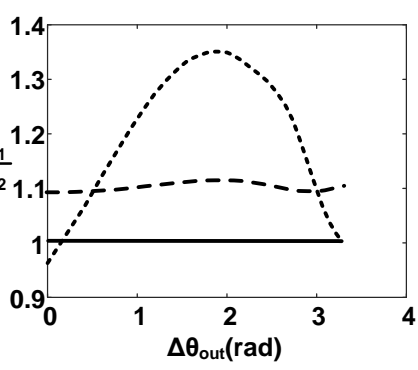

(b)

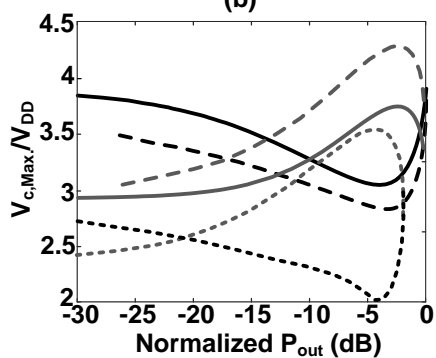

(d)

Figure 5.4: Ideal OEPA results for $0 \leq \Delta \theta_{\text {out }} \leq \pi$ for conventional design with $q_{1}=q_{2}=1.3$ (solid), for rotation with $q_{1}=1.4$ and $q_{2}=1.2$ (dashed) and for shiftrotation with $d=0.7, q_{1}=1.25$ and $q_{2}=1.02$ (dotted): (a) PA loads $Z_{1}$ (grey) and $Z_{2}$ (dark), (b) $\frac{V_{\text {out } 1}}{V_{\text {out } 2}}$ vs $\Delta \theta_{\text {out }}$, (c) efficiency vs normalized output power and (d) $V_{c, M a x}$. normalized to $V_{D D}$ for PA1 (grey) and PA2 (dark).

that the output voltage amplitude error first increases with $\Delta \theta_{\text {out }}$, and again reduces when $\Delta \theta_{\text {out }}$ approaches $\pi$, which therefore helps to improve the efficiency without sacrificing the OPDR.

Fig. 5.4c shows that the second compensation point is now shifted to almost $20 \mathrm{~dB}$ into back-off with lower switch voltage stress deep in back-off. However, by fine tuning of the parameters $d, q_{1}$ and $q_{2}$, the second compensation point can be easily shifted to any arbitrary back-off level between 10dB and 20dB. Furthermore, shifting the power contours can lower the maximum output power. For maximum output power and to benefit from the high back-off efficiency that the presented shift-rotation technique brings to the OEPA, one can tune the parameters $d$ and q dynamically according to the required instantaneous output power. 


\subsection{Second Order Effects}

\subsubsection{Switch conduction loss}

In the previous sections the switch was assumed to be ideal $(m=0)$. However, being implemented by transistors, the switch-on resistance $R_{o n}$ is non-zero which causes conduction loss for the time period during which the switch is closed. For a non-zero $m$, the output power and efficiency contours in Fig. 5.5a for a single class-E PA with $q=1.3$ and $d=1$ follow for a switch-on resistances with $m=0.05^{2}$.

Compared to the ideal load-pull contours shown in Fig. 5.1c, the elliptical shape of the efficiency contours is noticeable and, for real loads, the efficiencies between $3 \mathrm{~dB}$ to $6 \mathrm{~dB}$ back-offs are higher $(>85 \%)$ than the efficiency at peak output power $(<80 \%)$. Moreover, the elliptical shape of the efficiency contours seriously impacts the improvement that the rotation technique of section 5.4 brings to the OEPAs.

Fig. 5.5b shows the effect of reducing $q$ and $d$ on the power and efficiency contours; not only the power contours are shifted to the left, but that also the efficiency at backoff is improved. At e.g. the cross sections of the $-10 \mathrm{~dB}$ contours and the real axis in Fig. 5.5a and b, the efficiency is improved from almost $70 \%$ to $90 \%$. To benefit from these improved efficiency contours at back-off, the rotation technique can now be employed to optimally place the contours on the Smith chart for the actual load at back-off.

To demonstrate the effect of the shift-rotation technique in the case of lossy switch with $m=0.05$, we follow the same procedure as section 5.4 and derive the efficiency and the output power, yielding the plots in Fig. $5.5 \mathrm{c}$ and $\mathrm{d}$ for $B_{c}=\sin \left(\frac{\pi}{5}\right) / 2 R$ (correponding to compensation at $10 \mathrm{~dB}$ back-off for $m=0^{3}$ ). The results for the conventional OEPA design $\left(q_{1}=q_{2}=1.3\right.$ and $\left.d=1\right)$ are shown with solid-dark lines. For comparison, the efficiency of an OEPA with $q_{1}=q_{2}=1.3, d=1$ and $m=0.05$ with (ideal) real load modulation (zero imaginary part) is also shown in Fig. 5.5c and $\mathrm{d}$ with a solid grey curve. For this, the efficiency and the output power are obtained from Fig. 5.5a for real loads in the range of $[R, \infty]$. This curve also corresponds to the maximum reachable efficiency for techniques that rely on reducing the imaginary part of loads for the branch PAs at back-off to improve the back-off efficiency, e.g. the adaptive Chireix compensation elements technique [14] or 4-way OEPA systems [15].

Fig. $5.5 \mathrm{c}$ shows that compensation at $10 \mathrm{~dB}$ back-off for a conventional OEPA, with $q_{1}=q_{2}=1.3, d=1$, having a lossy switch can achieve the maximum reachable

\footnotetext{
${ }^{2}$ It is shown that $m$ only depends on the technology and the operation frequency [26]. For this thesis, at the frequency of interest $(1.8 \mathrm{GHz})$ for a cascode switch in $65 \mathrm{~nm}$ CMOS technology $m=0.05$ shows a fair agreement between theory and simulation results.

${ }^{3}$ Non-zero $\mathrm{m}(\mathrm{m}=0.05)$ slightly changes the power contours. It can be shown that for $\mathrm{m}=0.05$, $B_{c}=\sin \left(\frac{\pi}{5}\right) / 2 R$ corresponds to the compensation of the imaginary parts of the loads at (almost) $9 \mathrm{~dB}$ back-off. But, for simplicity, we ignore this small difference.
} 
efficiency ( $80 \%$ for $m=0.05$ ) at full power and near $10 \mathrm{~dB}$ back-off. The figure also shows that the rotation technique can improve the efficiency at more than $15 \mathrm{~dB}$ back-off. Comparing Fig. 5.5c and 5.4c also clearly shows that the switch conduction loss seriously impact the improvement of our proposed rotation technique as well as achieved by the adaptive tuning (or 4-way OEPA). On top of that, also additional losses of the tuning elements or due to extra components at the output can compromise this improvement. This last issue is also addressed in the measurements section.

Fig. 5.5d shows the effect of shift-rotation technique on the efficiency for two different settings Sett.1: $\left\{q_{1}, q_{2}, d\right\}=\{1.25,1.02,0.7\}$ and Sett.2: $\left\{q_{1}, q_{2}, d\right\}=\{1.18,0.95,0.5\}$ with dotted and dashed-dotted curves, respectively. The shift-rotation technique can significantly increase the efficiency in back-off: e.g. the efficiency is improved from less than $10 \%$ to more than $75 \%$ at $20 \mathrm{~dB}$ back-off (more than $\times 7.5$ improvement).

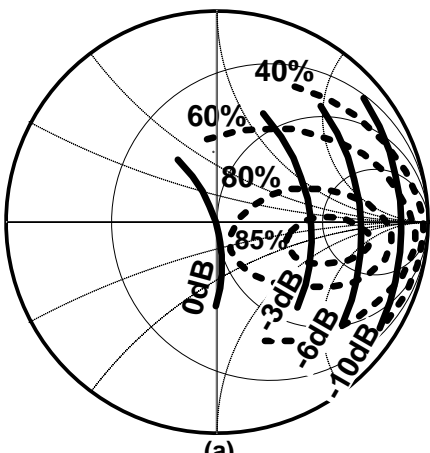

(a)

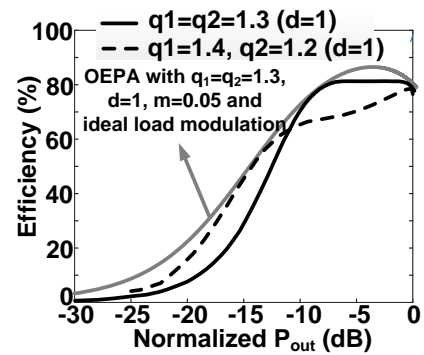

(c)

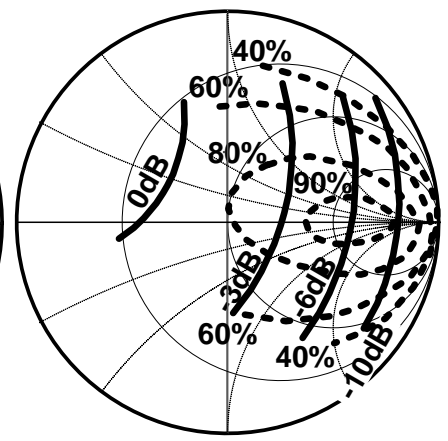

(b)

- q1 $=q 2=1.3(d=1)$

$\cdots q 1=1.25, q 2=1.02(d=0.7)$
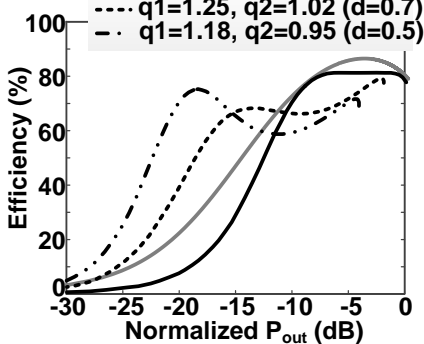

(d)

Figure 5.5: (a,b) The output power (solid) and the efficiency (dotted) contours for a single class-E PA with (a) $q=1.3, d=1$ and $m=0.05$ and (b) $q$ and $d$ are reduced to 1.1 and 0.7 , respectively. (c,d) The efficiency versus normalized output power for an OEPA with $m=0.05$ for the class-E branch PAs and with conventional design and compensation at 10dB back-off (solid-dark), with an ideal load modulation with zero imaginary part (solid-grey), with the rotation (dashed-dark), with the shift-rotation with setting Sett. 1 (dotted-dark) and the shift-rotation with setting Sett. 2 (dashed-dotted-dark). 


\subsubsection{Limited quality factor of the DC-feed inductor L}

Another important loss mechanism is due to the limited quality factor (Q) of the DCfeed inductor $L$, shown in Fig. 5.3a. This inductor can be a separate component [8] or it can be a part of the transformer-based combiner $[6,28]$. To study this effect, one can derive a new set of load-pull equations using $\mathrm{Q}$ as a parameter. This derivations were given in chapter 4 .

The resulting efficiency versus power curves for $m=0.05$ and $m=0.1$ and for quality factor value $Q=30$ are shown in Fig. 5.6 for the conventional OEPA design (solid curve) with compensation at $10 \mathrm{~dB}$ back-off, using the rotation technique (dashed curve) and for the shift-rotation with setting Sett.2 (dotted curve). For comparison, both graphs in Fig. 5.6 include a solid-grey curve that corresponds to the maximum efficiency of an OEPA with conventional design and ideal real load modulation, for the specific $Q$ and $m$ listed for each graph. Again, these curves represent the upper efficiency limit for any technique that optimizes efficiency by reducing the imaginary part of loads for the branch PAs at back-off [14], [15].

For the both cases, employing only the rotation technique can improve the efficiency to the maximum reachable efficiency (grey curve) at back-offs up to around $20 \mathrm{~dB}$. However, the improvement with respect to a conventional OEPA (black solid curve) almost vanishes for high $m$ or low $Q$. This is due to compression of the elliptical efficiency contours. However, the rotation technique can be used both to reduce the switch voltage stress at back-off and to improve the OPDR.

The proposed shift-rotation technique, however, improves the efficiency at backoff to levels significantly higher than the maximum reachable efficiency with real load modulation. Also, the improvement ratio increases with increasing switch conduction loss for a constant $Q$ (the absolute efficiencies are lower though). For instance, at $20 \mathrm{~dB}$ back-off, more than $\times 4.6$ and $\times 5.3$ higher efficiencies with respect to the conventional design are obtained for respectively, $m=0.05$ and 0.1 . It can be concluded that this shift-rotation technique is quite promising to improve the efficiency of an OEPA with an integrated combiner at high frequencies e.g. [28], where the switch conduction is the dominant loss mechanism.

\subsection{Implementation in 65nm CMOS technology}

The schematic of a single class-E branch PA and its driver stage is shown in Fig. 5.7a. Note that this system serves to demonstrate the performance increase of our shift-rotation technique, and does not aim at a specific transmit standard or application. The switch is implemented by a cascode structure employing a $1.2 \mathrm{~V}$ thin oxide transistor $(W / L=0.84 \mathrm{~mm} / 60 \mathrm{~nm})$ as switch transistor and a thick oxide $2.5 \mathrm{~V}$ transistor $(W / L=1.65 \mathrm{~mm} / 280 \mathrm{~nm})$ as cascode device. The cascode structure allows 


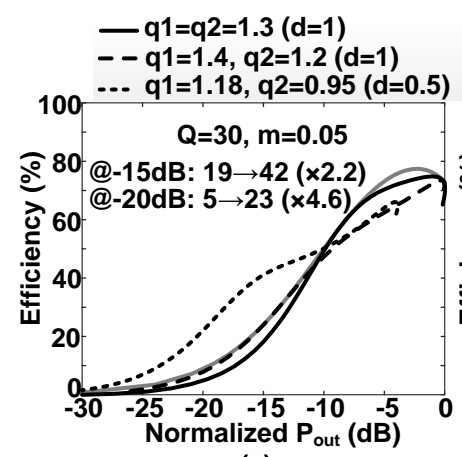

(a)

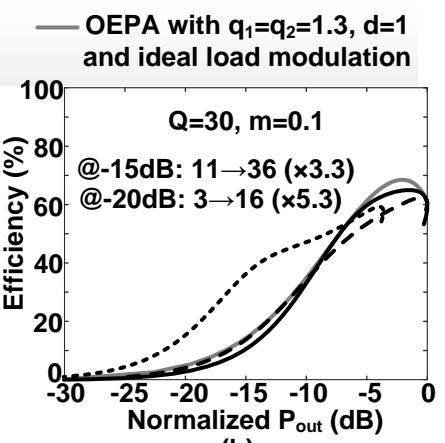

(b)

Figure 5.6: The efficiency versus normalized output power for a conventional OEPA with $q_{1}=q_{2}=1.3$ and compensation at 10dB back-off (solid-dark) and with an ideal load modulation (solid-grey), the rotation (dashed-dark), the shiftrotation with setting Sett. 2 (dotted-dark): (a) $m=0.05$ and (b) $m=0.1$.

$V_{c, \text { Max. up to }} 4 \mathrm{~V}$ for reliability reasons.

Using the K-design set elements for $q=1.3, d=1, m \approx 0.05$ and $\alpha=\beta=0$ and for $R=15 \Omega, \omega_{0}=2 \pi 1.8 \mathrm{GHz}$, yields $L=1.4 \mathrm{nH}, C=3.3 \mathrm{pF}$ and $X=0.5 \mathrm{nH}$. The DC-feed inductor $\mathrm{L}$ is implemented by two parallel bond-wire inductances to provide a relatively high quality factor $\mathrm{Q}(\approx 25)$. The tank capacitor $\mathrm{C}$ at the switching node was implemented with the drain-bulk and gate-drain parasitic capacitance of the cascode transistor. Moreover, the drain and the source of the cascode transistor were layed out close to each other to introduce some parasitic capacitance $C_{P, D S}$ to achieve slightly better efficiency [29]. Two cascaded inverters were used as the driver for the switch where the duty cycle can be controlled by the off-chip control voltage $V_{b}$.

Two switched capacitor banks with 4 control bits X1 and X2 were used at the switching nodes to tune the q parameter of the branch PAs independently. Circuit simulations in $65 \mathrm{~nm}$ CMOS technology suggest that a total switchable capacitance of $1.5 \mathrm{pF}$ is more than sufficient to employ the shift-rotation technique with shifting the contours up to $5 \mathrm{~dB}$. This $1.5 \mathrm{pF}$ capacitor is divided into 4 sections with $C_{1}=150 \mathrm{fF}$, $C_{2}=300 \mathrm{fF}, C_{3}=450 \mathrm{fF}$ and $C_{4}=600 \mathrm{fF}$, shown in Fig. 5.7a, being controlled with 4 control switches $S_{i}, i \in\{0,1,2,3\}$.

When each switch $S_{i}$ is off, it needs to tolerate the maximum voltage at the switching node which can be up to $4 \mathrm{~V}$. The switches $S_{i}$ are implemented by $2.5 \mathrm{~V}$ thick oxide transistors. There is a parasitic capacitance $C_{p, S_{i}}$ associated with the switch $S_{i}$, shown in Fig. 5.7a. The switches $S_{i}$ are sized to have $C_{p, S_{i}} \approx \frac{1}{4} C_{i}$ to make sure the maximum voltage across the switches $S_{i}$ does not exceed (almost) $3 \mathrm{~V}$. This switch-capacitor network at the switching node introduces extra capacitive loading 
at this node when all control bits are zero, which amounts to $0.3 \mathrm{pF}$ or $10 \%$ of the total drain capacitance. To compensate for this extra capacitance, the switch size was reduced by $10 \%$. This increases $m$ by $10 \%$ which impacts the (simulated) efficiency at maximum output power and at the back-off by less than $3 \%$.

To have the high and low level of the voltages at the driver output well defined, the driver and the main switch share the same ground and to reduce the bond-wire inductance at ground 6 parallel down-bonds (DBs) with minimum length were used (shown in Fig. 5.7b). The switches $S_{i}$ are controlled quasi-statically, driven from off-chip sources; no dynamic tuning is provided for this thesis. The microphotograph of the implemented branch class-E PA is shown in Fig. 5.7b.
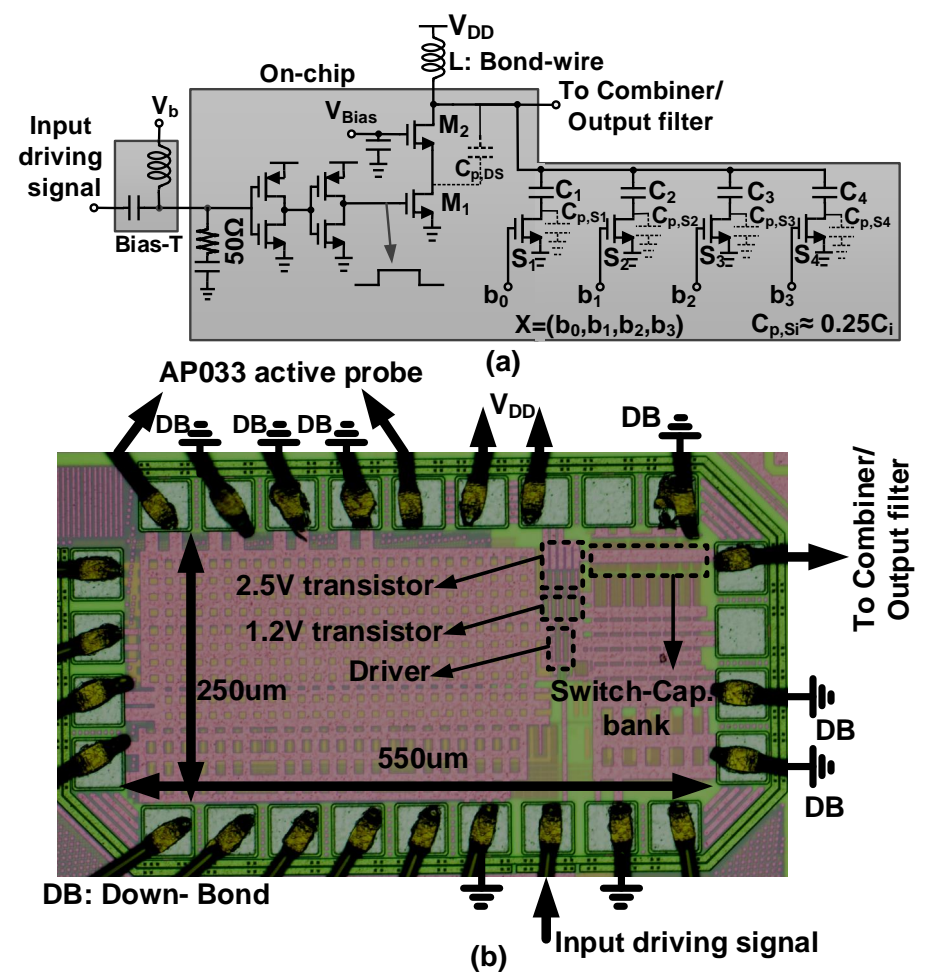

Figure 5.7: The branch class-E PAs (a) schematic, (b) chip microphotograph

The detailed implementation of the off-chip transmission-line based combiner and the output series filters $L_{0}-C_{0}$ are shown in Fig. 5.8a. The combiner is implemented on I-Tera MT RF substrate with a dielectric constant 3.45 and $0.5 \mathrm{~mm}$ thickness. The characteristic impedance $Z_{0}$ is obtained for $R=15 \Omega$ and $R_{L}=50 \Omega$ as $Z_{0}=$ $\sqrt{2 R R_{L}} \approx 39 \Omega$. The total series inductance at the branch class-E PAs outputs $\left(X+L_{0}\right)$ is implemented partly by the bond-wire inductance and partly by an off- 
chip component. The loaded quality factor of the output filter for $R=15 \Omega$ is roughly 5 . Due to the small thickness of the substrate and the relatively high loaded quality factor of the series filter, the parasitic capacitance $C_{p}$ becomes noticeable and changes the impedance level and impedance behavior $Z_{1,2}$ with $\Delta \theta_{\text {out }}$. To reduce this effect, a parallel quarter wavelength transmission-line terminated with a capacitor $C_{c}=$ $\frac{1}{C_{p} \omega^{2} Z_{c}^{2}}$ was used. The final designed PCB is shown in Fig. $5.8 \mathrm{~b}$ with the mounted branch class-E PAs.

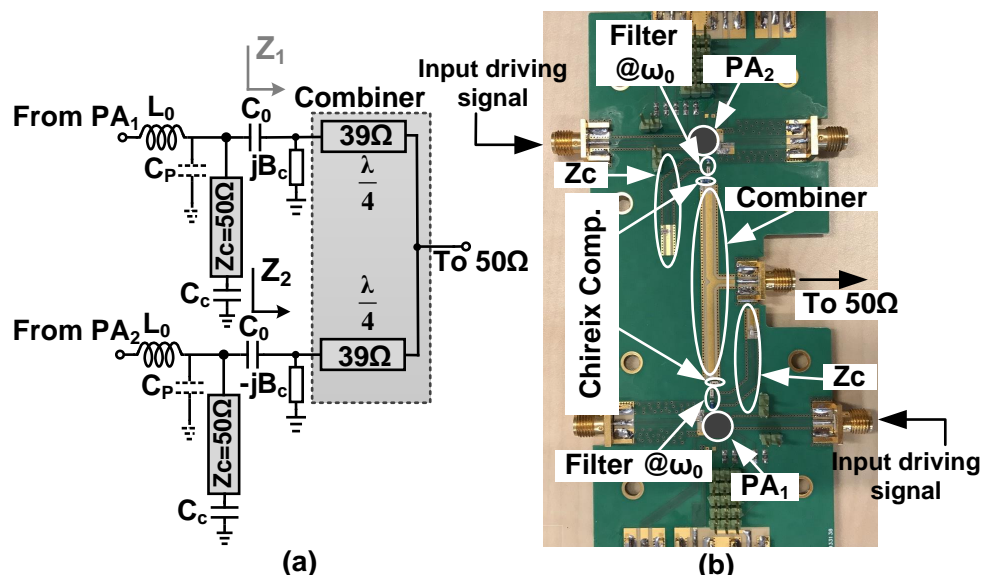

(a)

(b)

Figure 5.8: (a) The detailed schematic of the combiner, (b) the designed PCB

\subsection{Measurement Results}

Measured $P_{\text {out }}$ versus $\Delta \theta_{\text {in }}$ for 3 different conditions is shown in Fig. 5.9a for compensation of the imaginary part of the PA loads at $10 \mathrm{~dB}$ back-off. $+20.1 \mathrm{dBm}$ maximum output power $\left(P_{\text {out }, \text { Max. }}\right.$ ) is achieved for the conventional design from $1.25 \mathrm{~V}$ supply voltage at $1.8 \mathrm{GHz}$ while the mismatch between the branches limits OPDR to $40.2 \mathrm{~dB}$. Changing the PA settings to Sett. 1 , shifts the power contours by almost $-2.3 \mathrm{~dB}$ and rotates them which result in better than 50dB OPDR. Reducing duty cycles (setting 2 ), result in almost $-5.15 \mathrm{~dB}$ shift in power contours and in an OPDR improvement to more than $52 \mathrm{~dB}$. The improvement in the OPDR is (mainly) due to tuning the voltage ratio $\frac{V_{\text {out } 1}}{V_{\text {out } 2}}$ to cancel out the amplitude mismatch between the two branches, discussed in sections 5.3.1.

Fig. 5.9b shows the Drain Efficiency (DE) versus power back-off for 4 different conditions. Compensation at $20 \mathrm{~dB}$ back-off (dark-triangle (down)), by tuning $B_{c}$, with respect to compensation at $10 \mathrm{~dB}$ (grey-circle), brings less than $\times 1.15$ improvement at $15 \mathrm{~dB}$ back-off at the cost of reducing the efficiency at higher power levels. 
Employing adaptive elements [14] or using more components at the output to implement a 4-way system [15] will limit the improvement even more. However, as shown in Fig. 5.9b and c, the presented shift-rotation technique can effectively improve the DE and PAE.

Fig. 5.9b shows that measured DE at $P_{\text {out }, \text { Max }}$. for the conventional setting and compensation at $10 \mathrm{~dB}$ back-off is $65.3 \%$ and maximum $\mathrm{DE}$ is $68 \%$ at $1 \mathrm{~dB}$ backoff. PAE at $P_{\text {out }, \text { Max }}$, shown in Fig. 5.9c, is $60.7 \%$. By our proposed shift-rotation technique, $\times 2.5$ better $\mathrm{DE}$ and almost $\times 2$ better PAE at $15 \mathrm{~dB}$ back-off were achieved. PAE at $0 \mathrm{dBm}$ output power (20dB back-off) is also improved from $2 \%$ to more than $4 \%$. Therefore, to transmit $1 \mathrm{~mW}$ power the presented technique reduces supply power from $50 \mathrm{~mW}$ to less than $25 \mathrm{~mW}$.

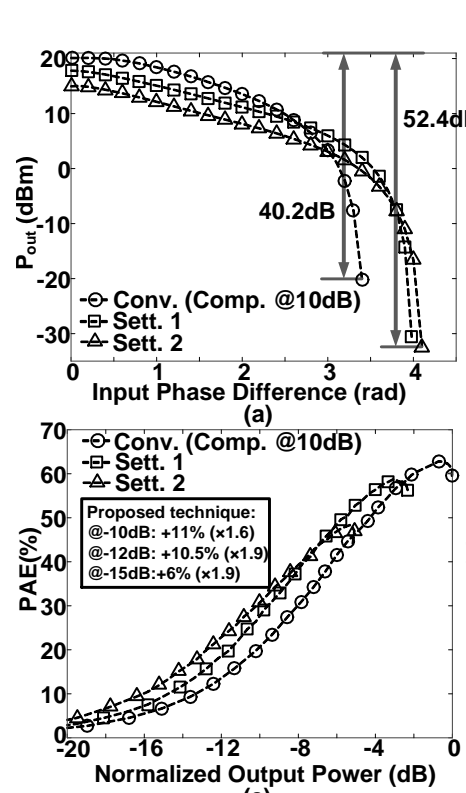

(c)

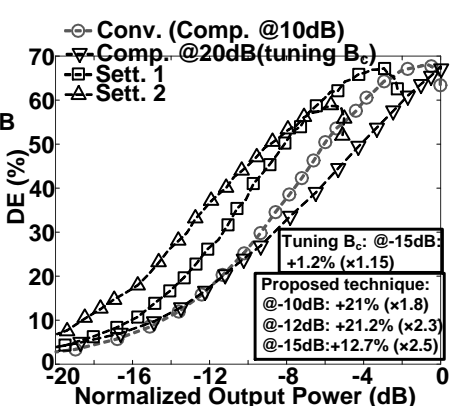

(b)

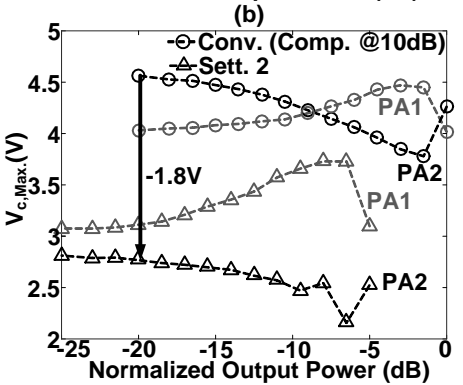

(d)

Figure 5.9: (a) Measured $P_{\text {out }}$ versus $\Delta \theta_{\text {in }}$ for compensation at 10dB back-off and 3 different settings ; conventional with $V_{b}=0.6 \mathrm{~V}, X_{1}=0000$ and $X_{2}=0000$ (corresponding to $\left.\left\{q_{1}, q_{2}, d\right\} \approx\{1.3,1.3,1\}\right)$, Sett.1 with $V_{b}=0.43 \mathrm{~V}, X_{1}=0011$ and $X_{2}=1010$ (corresponding to $\left\{q_{1}, q_{2}, d\right\} \approx\{1.25,1.02,0.7\}$ ) and and Sett.2 with $V_{b}=\mathbf{0 . 3 6} \mathbf{V}$, $X_{1}=\mathbf{0 0 0 0}$ and $X_{2}=1111$ (corresponding to $\left\{q_{1}, q_{2}, d\right\} \approx\{1.18,0.95,0.5\}$ ). (b) Measured DE for conventional compensation at 10dB back-off, Sett. 1 and 2 and for conventional setting and compensation at 20dB back-off (by tuning $B_{c}$ ). (c) Measured PAE for compensation at 10dB back-off and 3 different settings. (d) Measured $V_{c, M a x}$. for compensation at $10 \mathrm{~dB}$ back-off and 2 different settings.

Measured $V_{c, \text { Max }}$. for both PAs at $1.8 \mathrm{GHz}$ for compensation at $10 \mathrm{~dB}$ back-off are 
shown in Fig. 5.9d. For this, the transient waveforms were measured against the power back-off using an AP033 active probe and a 80GSa/s Agilent Oscilloscope. Maximum voltage, for the conventional configuration at maximum $P_{\text {out }}$ and for both switches, is almost $4 \mathrm{~V}$. For PA2, however, it increases to more than $4.5 \mathrm{~V}$ at $20 \mathrm{~dB}$ back-off which can cause reliability issues. Fig. $5.9 \mathrm{~d}$ shows that our proposed shift-rotation technique can significantly reduce the $V_{c, M a x}$. in power back-off; $-1.8 \mathrm{~V}$ reduction was measured for PA2 at 20dB back-off which reduces transistor degradation and hence improves the PA life-time.

We also measured the system performance across a frequency range from $1.78 \mathrm{GHz}$ to $1.88 \mathrm{GHz}$, see Fig. 5.10. The maximum $P_{\text {out }}$ at conventional setting is higher than $18.7 \mathrm{dBm}$ with more than $62 \%$ DE. Increasing the frequency from $1.8 \mathrm{GHz}$ to $1.88 \mathrm{GHz}$ results in $1.4 \mathrm{~dB}$ reduction in the maximum output power. Increasing the frequency, for both the branch class-E PAs the parameter q reduces which rotates and slightly shifts the power contours to the left (shown in Fig. 5.2a). Also, the positive residual impedance of the series filter at higher frequencies, rotates the PA loads toward the right-hand side of the Smith chart which further reduces the maximum output power. And finally, our transmission-line based power combiner is a narrow band combiner which further impacts the maximum output power by deviating from the center frequency [36].

The DE for conventional setting at 10dB back-off, shown in Fig. 5.10c reduces with increasing frequency due to the rotation of both efficiency contours in the same direction (counter-clockwise due to reduced $q$ ). However, the proposed technique improves the 10dB back-off efficiency to more than $34 \%$.

Executing the measurements with different samples, (almost) the same numbers for the maximum output power, efficiency at maximum output power and at $10 \mathrm{~dB}$ back-off were obtained. However, the OPDR is sensitive to the mismatch between the two samples. Fig. 5.10d shows the OPDR for three different PA realizations, each of these using their own set of (unselected) class-E PA ICs from the same batch. The OPDR for conventional design at center frequency $(1.8 \mathrm{GHz})$ is more than $37 \mathrm{~dB}$ and it reduces sharply with deviating the frequency from $1.8 \mathrm{GHz}$. The OPDR reduces to almost $11 \mathrm{~dB}$ (solid curve) at $1.88 \mathrm{GHz}(4.45 \%$ deviation from $1.8 \mathrm{GHz})$. This is mainly due to the residual impedance of the filter at frequencies different from the $\omega_{0}$, discussed in section 5.3.1. The presented technique, however, improves the OPDR to more than $49 \mathrm{~dB}$ at $1.8 \mathrm{GHz}$ and to more than $34.8 \mathrm{~dB}$ across $100 \mathrm{MHz}$ bandwidth. As discussed in section 5.4.1, toward higher frequencies we rotate the contours of PA2 in reverse direction by increasing $q_{2}$. For this $X_{2}$ is reduced from 1111 at $1.8 \mathrm{GHz}$ to 0000 at $1.88 \mathrm{GHz}$ for the same $V_{b}$. Toward lower frequencies, however, we need to rotate the contours more, in the same direction that we rotate at $1.8 \mathrm{GHz}$. This is done by slightly increasing $d$.

The designed OEPA was also characterized using single carrier $1.8 \mathrm{GHz}$ amplitude 

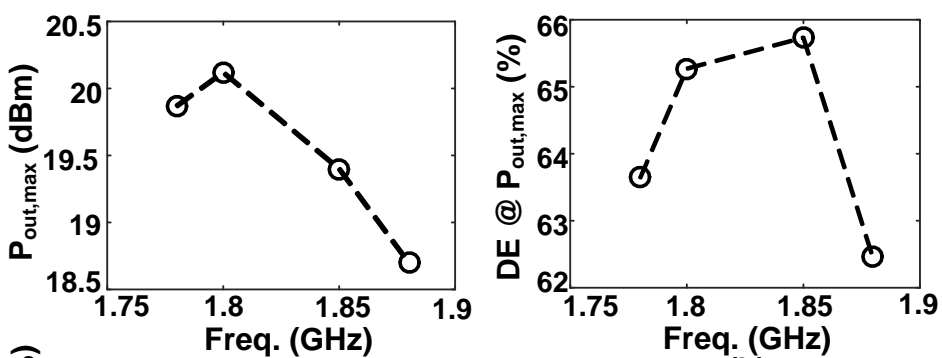

(a)

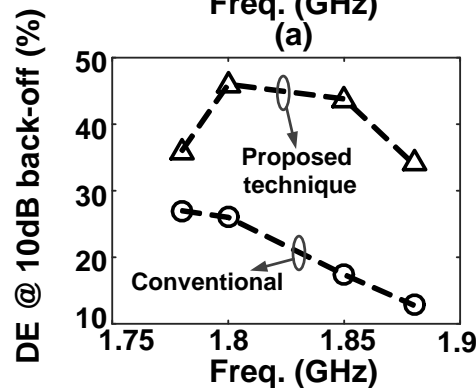

(c)

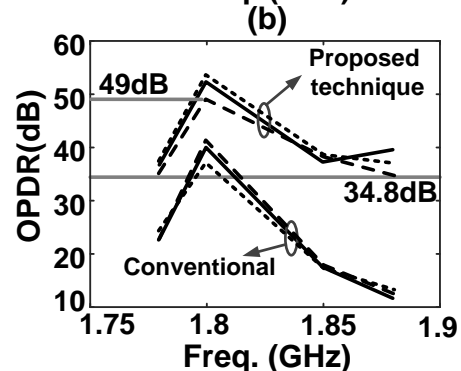

(d)

Figure 5.10: (a) Measured maximum $P_{\text {out }}$, (b) DE at maximum $P_{\text {out }}$, (c) DE at $10 \mathrm{~dB}$ back-off and (d) OPDR (for three different PA realizations, each with two (unselected) class-E PA ICs) versus frequency for conventional design with $V_{b}=0.6 \mathrm{~V}, X_{1}=0000$ and $X_{2}=0000$ and for the proposed technique with $V_{b}=0.43 \mathrm{~V}$, $X_{1}=0000$ and $X_{2}=1111$ at $1.78 \mathrm{GHz}$, with $V_{b}=0.36 \mathrm{~V}, X_{1}=0000$ and $X_{2}=1111$ at $1.8 \mathrm{GHz}$, with $V_{b}=0.36 \mathrm{~V}, X_{1}=0000$ and $X_{2}=1000$ at $1.85 \mathrm{GHz}$ and with $V_{b}=0.36 \mathrm{~V}$, $X_{1}=0000$ and $X_{2}=0000$ at $1.88 \mathrm{GHz}$.

modulated signals. For this first the output voltage amplitude ( $V_{\text {out }}$ in Fig .5.3a) and phase are measured versus $\Delta \theta_{i n}$ for 2 different settings, shown in Fig. 5.11a to implement a memory-less Digital Pre-Distortion (DPD).

The output power spectral density (PSD) and symbol constellation for a single carrier $7 \mathrm{~dB}$ PAPR 64QAM amplitude modulated signal with $5 \mathrm{MSym} / \mathrm{s}$ symbol rate are shown in $5.11 \mathrm{~b}$ and $\mathrm{c}$ for two cases, without DPD and using a memory-less DPD. For this, the polar representations (the normalized envelope and the angle) of the time domain base-band IQ signals were generated in Matlab. Then, we constructed two carrier signals (at $1.8 \mathrm{GHz}$ ) where the phase difference between the two carries was obtained from inverse cosine of the envelope (non-DPD case) and for the DPD, the inverse of plots shown in Fig. 5.11a (in dark). The common mode phase of the carriers is equal to the angle of the IQ signal (no-DPD). However, for the DPD, the output voltage phases, shown in Fig. 5.11a (in grey), were derived from the common mode phase of the carriers. The two carrier signals were then uploaded to a $12 \mathrm{GS} / \mathrm{s}$ M9502A Agilent Arbitrary Waveform Generator with two output channels to drive 


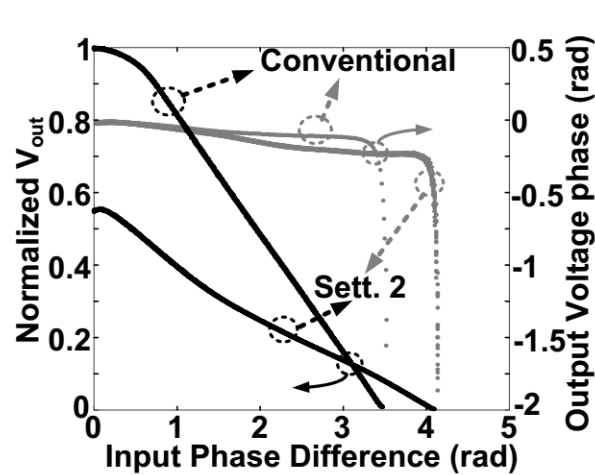

(a)

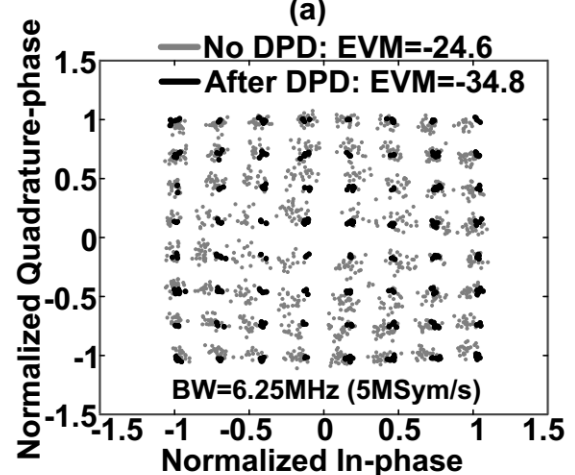

(c)

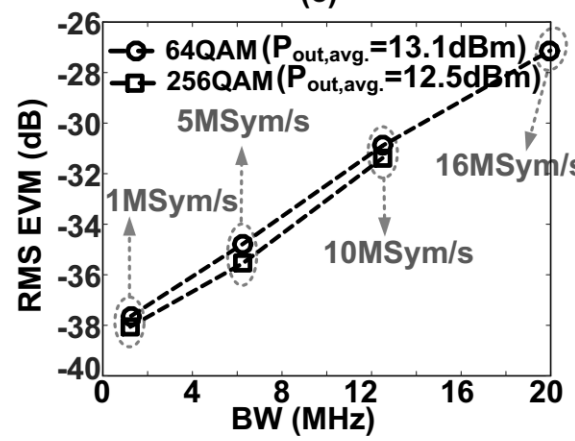

(e)
$P_{\text {out,avg. }}=13.1 \mathrm{dBm}$ DE=41.8\% PAE=33.6\%

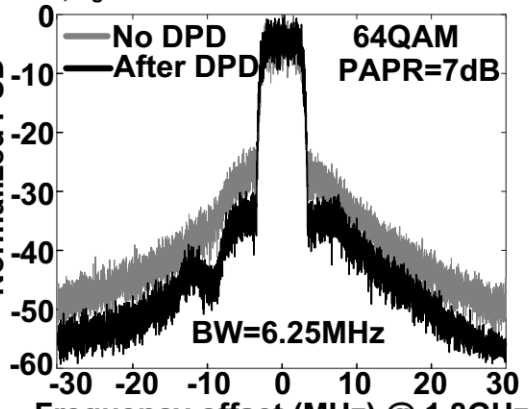

Frequency offset $(\mathrm{MHz}) @ 1.8 \mathrm{GHz}$

(b)

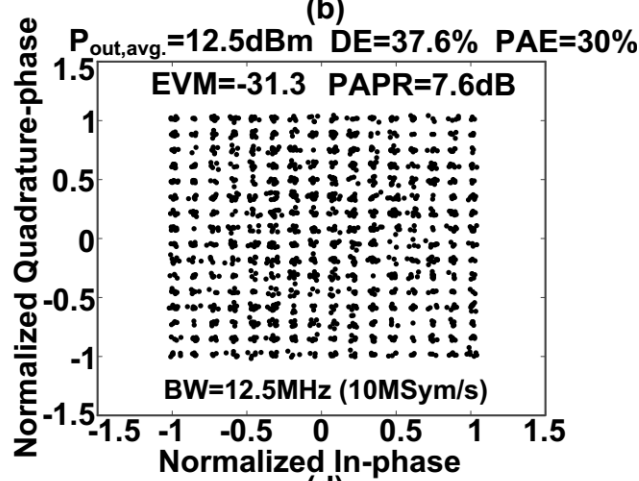

(d)

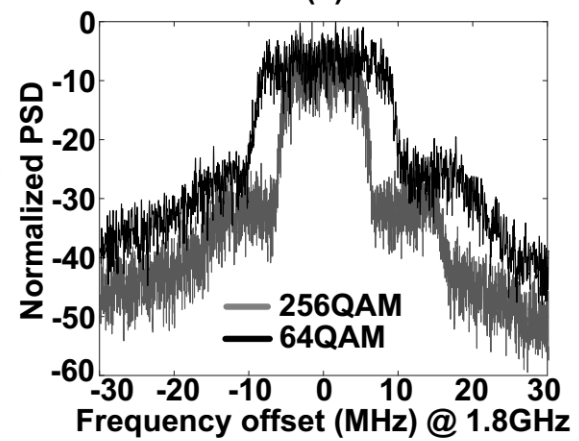

(f)

Figure 5.11: (a) Measured output voltage $\left(V_{\text {out }}\right)$ amplitude (dark) and phase (grey) versus input phase difference for 2 different settings at $1.8 \mathrm{GHz}$. The effect of DPD on PSD (b) and symbol constellation (c) for the conventional setting and for a 64QAM amplitude modulated signal. (d) Measured symbol constellation (after DPD) for the conventional setting and for a $1.8 \mathrm{GHz} 256 \mathrm{QAM}$ modulation. (e) EVM versus modulation BW for the conventional setting. (f) Measured PSD for the 256QAM and 64QAM modulations with $12.5 \mathrm{MHz}$, respectively $20 \mathrm{MHz}$ modulation bandwidths. 
Table 5.1: Measured efficiency (after DPD) for 7.6dB PAPR 256QAM modulation with $6.25 \mathrm{MHz}(5 \mathrm{Msym} / \mathrm{s})$ and $12.5 \mathrm{MHz} \mathrm{BW}(10 \mathrm{Msym} / \mathrm{s})$ and different average $P_{\text {out }}$.

\begin{tabular}{|c|c|c|c|c|c|c|c|c|}
\hline \multirow{2}{*}{ P $_{\text {out }, \text { avg. }}$} & \multicolumn{2}{|c|}{ DE $(\%)$} & \multicolumn{2}{|c|}{ PAE $(\%)$} & \multicolumn{4}{c|}{ EVM $(\mathrm{dB})$} \\
\cline { 2 - 9 } & Con. & Set.2 & Con. & Set.2 & Con. & Set.2 & Con. & Set.2 \\
\hline $12.5 \mathrm{dBm}$ & 37.6 & - & 30 & - & -35.6 & - & -31.3 & - \\
\hline $7.5 \mathrm{dBm}$ & 14.8 & 29 & 11.3 & 17.9 & -33.4 & -36.1 & -30.3 & -31.5 \\
\hline $2.5 \mathrm{dBm}$ & 4.2 & 10.1 & 3.7 & 6.3 & -32.1 & -35.9 & -30.1 & -31.2 \\
\hline
\end{tabular}

the two class-E branch PAs. Fig. 5.11c shows that, for the conventional design, DPD improves the RMS EVM from $-24.6 \mathrm{~dB}$ to $-34.8 \mathrm{~dB}$ with $13.1 \mathrm{dBm}$ average $P_{\text {out }}$ ( $P_{\text {out }, \text { avg. }}$ ), $41.8 \%$ DE and $33.6 \%$ PAE. The measured symbol constellation (using DPD) for a single carrier 7.6dB PAPR 256QAM amplitude modulated signal with $10 \mathrm{MSym} / \mathrm{s}$ symbol rate and for the conventional setting is also shown in Fig. 5.11d. $+12.5 \mathrm{dBm}$ average output power with $37.6 \%$ DE and $30 \%$ PAE was measured at 31.3dB RMS EVM level. Dynamic tuning of parameters d and q was not implemented in this thesis. However, if dynamic tuning would be used to tune the parameters $\mathrm{d}$ and $q$ according to the instantaneous output power level, it can be estimated that the PA then could provide about $48 \%{ }^{4} \mathrm{DE}$ for $7.6 \mathrm{~dB}$ PAPR $256 \mathrm{QAM}$ amplitude modulated signals.

Measured EVM as a function of the modulation bandwidth is shown in Fig. 5.11e for both 64QAM and 256QAM modulations at a constant average output power. The EVM increases with increasing bandwidth (signal symbol rate). The limited bandwidth of the OEPAs is both due to 10 15x bandwidth expansion that comes with the non-linear SCS operation in outphasing mode [35] and due to the limited bandwidth of the output series filter and the combiner. Ways to improve the modulation bandwidth include a lower loaded quality factor for the output filters and using transformer based combiners [36]. The output PSDs for the 256QAM and 64QAM amplitude modulations with $12.5 \mathrm{MHz}$, respectively $20 \mathrm{MHz}$ modulation bandwidths are shown in Fig. 5.11f.

Finally, we measured the OEPA performance with 256QAM modulated signals with different $P_{\text {out,avg }}$. to demonstrate the effect of the proposed technique in the efficiency improvement of the OEPA at back-off. A summary of the measured DEs and PAEs are given in Table 5.1. At $5 \mathrm{~dB}$ back-off $\left(P_{\text {out }, \text { avg }}=7.5 \mathrm{dBm}\right)$ the $\mathrm{DE}$ and PAs are improved from $14.8 \%$ and $11.3 \%$ for the conventional design to $29 \%(\times 1.96)$ and $17.9 \%(\times 1.6)$ at a better EVM level. At $10 \mathrm{~dB}$ back-off $\left(P_{\text {out }, \text { avg. }}=2.5 \mathrm{dBm}\right) \mathrm{DE}$ 
and PAE are improved by $\times 2.4$ and $\times 1.7$ with better RMS EVM.

In Table 5.2 , the measured results at $1.8 \mathrm{GHz}$ are benchmarked against the other CMOS PAs. The designed PA at conventional settings provides the best DE and PAE for single tone excitation at maximum output power. Furthermore, the presented technique improves the DE at $12 \mathrm{~dB}$ back-off to more than 1.7 times better than other published works with a comparable PAE. The presented demonstrator PA has $20.1 \mathrm{dBm}$ maximum output power which is lower than some other reported works. However, the outphasing theory and the back-off efficiency improvement approach described in the chapter are not limited to a specific frequency or power level or technology: on purpose we (re)normalize voltages, power levels, impedances and use Smith chart representations to be as independent from frequency, power and technology as possible. Scaling our PA to achieve higher than 20.1dBm maximum output power levels in first order (ideally) has no impact on DE and PAE numbers $[37]^{5}$. Section 5.5.B already discussed the impact of the operating frequency on the merits of the efficiency enhancement technique.

All the measured efficiency numbers include the loss of the DC-feed inductor L (with $\mathrm{Q}=25)$, output bondwire inductance loss $(1 \mathrm{nH}$ with $\mathrm{Q}=15)$, the loss of the offchip inductor $L_{0}$ (which has a series resistance $0.5 \Omega$ ) and the combiner loss $(0.3 \mathrm{~dB})$. Therefore, replacing the PCB-based combiner with an on-chip transformer based counterpart will not considerably affect the efficiency [39]. Due to lack of relevant data in literature, we cannot benchmark the effect of the presented technique on $V_{c, \text { Max }}$. against the other efficiency improvement techniques.

\subsection{Conclusion}

A simple analysis of the Outphasing class-E PAs (OEPAs) based on the load-pull analyses of the class-E PAs was given. The results of the study, then, further were used to rotate and shift the power contours and to rotate the efficiency contours to improve OEPAs performance and reliability aspects. Measurements in 65nm CMOS technology showed more than $\times 2$ drain efficiency improvement at deep back-off for single tone excitation at $1.8 \mathrm{GHz}$ as well as for $7.6 \mathrm{~dB}$ PAPR $12.5 \mathrm{MHz} 256 \mathrm{QAM}$ amplitude modulated signals.

\footnotetext{
${ }^{4}$ The average efficiency was estimated from the integral $\int_{-\infty}^{0} \operatorname{pdf}(\mathrm{BF}) \cdot \max (\mathrm{DE}(\mathrm{BF})) \mathrm{d}(\mathrm{BF})$ where $\operatorname{pdf}(\mathrm{BF})$ is the probability density function of back-off level $\mathrm{BF}$ for a modulated signal and $\max (\mathrm{DE}(\mathrm{BF}))$ is the maximum achievable efficiency at back-off level BF for the three different settings shown in Fig. 5.9b.

${ }^{5}$ The matching network should also be adapted with the scaling; if a matching network with a higher quality factor were to be used to get higher output power (from a low-voltage PA), the Bode-Fano theorem [38] shows a limitation of the bandwidth. This is however a secondary effect, not inherent to the presented efficiency enhancement technique.
} 
Table 5.2: Performance comparison

\begin{tabular}{|c|c|c|c|c|c|c|c|c|c|c|c|}
\hline & [11] & [12] & [27] & [29] & \multicolumn{2}{|c|}{$[30]$} & {$[31]$} & [32] & [33] & \multicolumn{2}{|c|}{ This Work } \\
\hline CMOS Technology & 0.13 (um) & $65(\mathrm{~nm})$ & $40(\mathrm{~nm})$ & $45(\mathrm{~nm})$ & \multicolumn{2}{|c|}{$65(\mathrm{~nm})$} & $40(\mathrm{~nm})$ & $65(\mathrm{~nm})$ & $90(\mathrm{~nm})$ & \multicolumn{2}{|c|}{$65(\mathrm{~nm})$} \\
\hline Topology & OEPA & OEPA & OEPA & ODPA(e) & Class-G & DLTM(f) & Doherty & Polar & OBPA(j) & \multicolumn{2}{|c|}{ OEPA } \\
\hline Combiner & On-chip & Off-chip & On-chip & Off-chip & \multicolumn{2}{|c|}{ On-chip } & On-chip & On-chip & Off-chip & \multicolumn{2}{|c|}{ Off-chip } \\
\hline Frequency $(\mathrm{GHz})$ & 1.85 & 2.4 & 5.9 & $0.9-2.4$ & \multicolumn{2}{|c|}{2.4} & 1.9 & 2.4 & 0.9 & \multicolumn{2}{|c|}{1.8} \\
\hline Supply (V) & 2.8 & $2.5-0.85$ (a) & 1.2 & 1.2 & \multicolumn{2}{|c|}{$2.8-1.55(\mathrm{~g})$} & 1.5 & 1.2 & 2.5 & \multicolumn{2}{|c|}{1.25} \\
\hline $\mathrm{P}_{\text {out } . M a x}(\mathrm{dBm})$ & 29.7 & 27.7 & 22.2 & $25-25$ & \multicolumn{2}{|c|}{24.6} & 28 & 22.8 & 20 & \multirow{2}{*}{\multicolumn{2}{|c|}{\begin{tabular}{|l|}
20.1 \\
65.3
\end{tabular}}} \\
\hline DE at $P_{\text {out, }}$ Max & 39.8 & NR(b) & 49.2 & $60-52$ & \multicolumn{2}{|c|}{39} & NR & 46 & NR & & \\
\hline PAE at $P_{\text {out,Max }}$ & 36.9 & 45 & 34.9 & $55-45$ & \multicolumn{2}{|c|}{ NR } & 34 & NR & 56 & \multicolumn{2}{|c|}{60.7} \\
\hline $\mathrm{DE} / \mathrm{PAE}$ at $12 \mathrm{~dB}$ & NR & NR & $<18$ (d) & $20 / 14$ & \multirow{2}{*}{\multicolumn{2}{|c|}{$\begin{array}{l}21.8 \\
\text { /NR }\end{array}$}} & NR & NR & NR & \multirow{2}{*}{\multicolumn{2}{|c|}{$\begin{array}{l}37.2 \\
/ 22.1\end{array}$}} \\
\hline back-off (\%) & $/ \approx 5(\mathrm{c})$ & $1<22$ (c) & $<13$ & $12 / 8(\mathrm{c})$ & & & $/ 19.7$ & /NR & $1<20$ & & \\
\hline $\begin{array}{c}\text { Signal } \\
\text { (PAPR }(\mathrm{dB}))\end{array}$ & $\begin{array}{l}\text { LTE } \\
(7.5)\end{array}$ & $\begin{array}{c}\text { OFDM } \\
(7.5)\end{array}$ & $\begin{array}{c}\text { 64QAM } \\
(7.2)\end{array}$ & $\begin{array}{l}\text { LTE } \\
(6)\end{array}$ & $\begin{array}{l}\text { 64QAM } \\
\text { (7) }\end{array}$ & $\begin{array}{c}\text { 256QAM } \\
(7.3)\end{array}$ & $\begin{array}{c}\text { 16QAM } \\
(8.36)\end{array}$ & $\begin{array}{c}\text { 64QAM } \\
(6.5)\end{array}$ & $\begin{array}{c}\text { WCDMA } \\
\text { NR }\end{array}$ & $\begin{array}{l}\text { 64QAM } \\
\text { (7) }\end{array}$ & $\begin{array}{c}\text { 256QAM } \\
\text { (7.6) }\end{array}$ \\
\hline $\mathrm{BW}(\mathrm{MHz})$ & 10 & 20 & 20 & $10-10$ & NR(h) & NR(i) & 20 & 20 & 5 & $12.5 / 20$ & $6.25 / 12.5$ \\
\hline Fractional BW (\%) & 0.54 & 0.83 & 0.34 & $1.11-0.42$ & NR & $\mathrm{NR}$ & 1.05 & 0.83 & 0.55 & $0.7 / 1.11$ & $0.35 / 0.7$ \\
\hline $\mathrm{P}_{\text {out,avg. }}(\mathrm{dBm})$ & 24.7 & 20.2 & 16.4 & 18.9 & 17.6 & 17.3 & 23.4 & 16.8 & 13 & 13.1 & 12.5 \\
\hline DE at $P_{\text {out, avg. }}$ & NR & 31.9 & 23.3 & NR-NR & 27.5 & 26.7 & NR & 24.5 & NR & 41.8 & 37.6 \\
\hline PAE at $P_{\text {out }}$ avg. & 20.8 & 27.6 & 16.1 & $32-22$ & NR & NR & 23.3 & 19.3 & 30 & 33.6 & 30 \\
\hline RMS EVM(dB) & -30.5 & -31.3 & -30 & NR & -25.6 & -30.4 & -23 & -28 & -16.6 & $-30.9 /-27.1$ & $-35.6 /-31.3$ \\
\hline$\frac{V_{\text {cmax }}}{V_{\text {DP }}}$ at $P_{\text {out }}, \operatorname{Max}$ & NR & NR & NR & NR & \multirow{2}{*}{\multicolumn{2}{|c|}{$\begin{array}{l}\mathrm{NR} \\
/ \mathrm{NR}\end{array}$}} & NR & NR & NR & \multirow{2}{*}{\multicolumn{2}{|c|}{$\begin{array}{l}<3.3 \\
K<2.6\end{array}$}} \\
\hline / $15 \mathrm{~dB}$ back-off & /NR & /NR & /NR & /NR & & & /NR & /NR & /NR & & \\
\hline
\end{tabular}

(a) Multi-level supply, (b) Not Reported, (c) obtained from publication figures, (d) $\mathrm{DE}=18 \%$ and $\mathrm{PAE}=13 \%$ at $9 \mathrm{~dB}$ back-off, obtained from publication
figures, (e) Outphasing class-D PA, (f) Dynamic Load Trajectory Manipulation, (g) full and half $V_{D D}$ mode, (h) 20MSym/s reported symbol rate, (i) $10 \mathrm{MSym} / \mathrm{s}$ reported symbol rate, (j) Outphasing class-B PA.

Class-E PAs (and the outphasing systems that employ class-E PAs as the branch amplifiers) are tuned amplifers and hence are optimized for narrow-band applications. However, measurement results across a wide frequency range $1.78 \mathrm{GHz}-1.88 \mathrm{GHz}$ showed that the presented technique can improve the OPDR to more than $34.8 \mathrm{~dB}$ with at least $34 \% \mathrm{DE}$ at $10 \mathrm{~dB}$ back-off in this frequency range. 


\section{Bibliography}

[1] A. Ghahremani, A. J. Annema and B. Nauta, "Outphasing Class-E Power Amplifiers: From Theory to Back-Off Efficiency Improvement," in IEEE JSSC, vol. 53, no. 5, pp. 1374-1386, May 2018.

[2] F.H. Raab, "Idealized operation of the class-E tuned power amplifier," in Circuits and Systems, IEEE Transactions on, vol. 24, no. 12, pp. 725-735, Dec. 1977.

[3] S. C. Cripps, "RF Power Amplifiers for Wireless Communications," Norwood, MA: Artech House, pp. 113-143, 219-249.

[4] R. Beltran, F. H. Raab and A. Velazquez, "HF outphasing transmitter using classE power amplifiers," in IEEE MTT-S International Microwave Symposium Digest, 2009, pp. 757-760.

[5] N. O. Sokal and A. D. Sokal, "Class E-A new class of high-efficiency tuned singleended switching power amplifiers," in IEEE Journal of Solid-State Circuits, vol. 10, no. 3, pp. 168-176, June 1975.

[6] M. P. van der Heijden, M. Acar, J. S. Vromans and D. A. Calvillo-Cortes, "A 19W high-efficiency wide-band CMOS-GaN class-E Chireix RF outphasing power amplifier," 2011 IEEE MTT-S International Microwave Symposium, Baltimore, MD, 2011, pp. 1-4.

[7] R. Sadeghpour, A. Nabavi, "Design Procedure of Quasi-Class-E Power Amplifier for Low-Breakdown-Voltage Devices," Circuits and Systems I: Regular Papers, IEEE Transactions on, vol. 61, no. 5, pp. 1416-1428, May 2014.

[8] A. Ghahremani, A. J. Annema and B. Nauta, "A 20dBm outphasing class E PA with high efficiency at power back-off in 65nm CMOS technology," in IEEE Radio Frequency Integrated Circuits Symposium (RFIC), 2017, pp. 340-343.

[9] I. Aoki, S. Kee, R. Magoon, R. Aparicio, F. Bohn, J. Zachan, G. Hatcher, D. McClymont and A. Hajimiri, "A Fully-Integrated Quad-Band GSM/GPRS CMOS 
Power Amplifier," Solid-State Circuits, IEEE Journal of, vol. 43, no. 12, pp. 27472758, Dec. 2008.

[10] M. Ozen, M. van der Heijden, M. Acar, R. Jos and C. Fager, "A Generalized Combiner Synthesis Technique for Class-E Outphasing Transmitters," in IEEE Transactions on Circuits and Systems I: Regular Papers, vol. 64, no. 5, pp. 11261139, May 2017.

[11] N. Singhal, H. Zhang and S. Pamarti, "A Zero-Voltage-Switching Contour-Based Outphasing Power Amplifier," in IEEE Transactions on Microwave Theory and Techniques, vol. 60, no. 6, pp. 1896-1906, June 2012.

[12] S. Shim and S. Pamarti, "A 1.85GHz CMOS power amplifier with zero-voltageswitching contour-based outphasing control to improve back-off efficiency," 2015 IEEE MTT-S International Microwave Symposium, Phoenix, AZ, 2015, pp. 1-4.

[13] P. A. Godoy, S. Chung, T. W. Barton, D. J. Perreault and J. L. Dawson, "A 2.4-GHz, 27-dBm Asymmetric Multilevel Outphasing Power Amplifier in 65-nm CMOS," in IEEE Journal of Solid-State Circuits, vol. 47, no. 10, pp. 2372-2384, Oct. 2012.

[14] J. Qureshi, R. Liu, A. J. M. de Graauw, M. P. van der Heijden, J. Gajadharsing and L. C. N. de Vreede, "A highly efficient chireix amplifier using adaptive power combining," in IEEE MTT-S International Microwave Symposium Digest, 2008, pp. 759-762.

[15] D. J. Perreault, "A New Power Combining and Outphasing Modulation System for High-Efficiency Power Amplification," in IEEE Trans. on Circuits and Systems I: Regular Papers, vol. 58, no. 11, Aug. 2011.

[16] M. P. van der Heijden and M. Acar, "A Radio-Frequency Reconfigurable CMOSGaN Class-E Chireix Power Amplifier," IEEE MTT-S Int'l. Microwave Symp. Digest, June 2014.

[17] 3GPP TS 36.101 V14.3.0, "Evolved Universal Terrestrial Radio Access (EUTRA); User Equipment (UE) radio transmission and reception", April 2017.

[18] H. Wang et al., " A Highly-Efficient Multi-Band Multi-Mode All-Digital Quadrature Transmitter," in IEEE Transactions on Circuits and Systems I: Regular Papers, vol. 61, no. 5, pp. 1321-1330, May 2014.

[19] H. S. Chen, Y. K. Hsieh and L. H. Lu, "A 5.5-GHz multi-mode power amplifier with reconfigurable output matching network," 2015 IEEE Radio Frequency Integrated Circuits Symposium (RFIC), Phoenix, AZ, 2015, pp. 203-206. 
[20] N. Singhal, N. Nidhi, R. Patel and S. Pamarti, "A Zero-Voltage-Switching Contour-Based Power Amplifier With Minimal Efficiency Degradation Under Back-Off," in IEEE Transactions on Microwave Theory and Techniques, vol. 59, no. 6, pp. 1589-1598, June 2011.

[21] M. Ozen, R. Jos, C. M. Andersson, M. Acar and C. Fager, "High-Efficiency RF Pulsewidth Modulation of Class-E Power Amplifiers," in IEEE Transactions on Microwave Theory and Techniques, vol. 59, no. 11, pp. 2931-2942, Nov. 2011.

[22] M. Acar, A.J. Annema, B. Nauta, "Analytical Design Equations for Class-E Power Amplifiers," Circuits and Systems I: Regular Papers, IEEE Transactions on, vol .54, no. 12, pp. 2706-2717, Dec. 2007.

[23] R. A. Beltran and F. H. Raab, "Simplified analysis and design of outphasing transmitters using class-E power amplifiers," 2015 IEEE Topical Conference on Power Amplifiers for Wireless and Radio Applications (PAWR), San Diego, CA, 2015, pp. 1-3.

[24] K. Tom and M. Faulkner, "Load pull methodology to characterise class-E outphasing power amplifiers," in IET Microwaves, Antennas and Propagation, vol. 6, no. 4, pp. 387-392, March 202012.

[25] R. Zhang, M. Acar, M. P. van der Heijden, M. Apostolidou and D. M. W. Leenaerts, "Generalized Semi-Analytical Design Methodology of Class-E Outphasing Power Amplifier," in IEEE Transactions on Circuits and Systems I: Regular Papers, vol. 61, no. 10, pp. 2951-2960, Oct. 2014.

[26] M. Acar, A. J. Annema and B. Nauta, "Variable-Voltage Class-E Power Amplifiers," in IEEE MTT-S International Microwave Symposium, 2007, pp. 1095-1098.

[27] M. Acar, A. J. Annema and B. Nauta, "Generalized Analytical Design Equations for Variable Slope Class-E Power Amplifiers," in IEEE International Conference on Electronics, Circuits and Systems, 2006, pp. 431-434.

[28] Z. Hu, L. C. N. de Vreede, M. S. Alavi, D. A. Calvillo-Cortes, R. B. Staszewski and S. He, "A 5.9 GHz RFDAC-based outphasing power amplifier in 40-nm CMOS with $49.2 \%$ efficiency and $22.2 \mathrm{dBm}$ power," in IEEE Radio Frequency Integrated Circuits Symposium (RFIC), 2016, pp. 206-209.

[29] O. Lee et al., " A Charging Acceleration Technique for Highly Efficient Cascode Class-E CMOS Power Amplifiers," in IEEE Journal of Solid-State Circuits, vol. 45, no. 10, pp. 2184-2197, Oct. 2010. 
[30] L. Ding, J. Hur, A. Banerjee, R. Hezar and B. Haroun, "A 25 dBm Outphasing Power Amplifier With Cross-Bridge Combiners," in IEEE Journal of Solid-State Circuits, vol. 50, no. 5, pp. 1107-1116, May 2015.

[31] S. Hu, S. Kousai and H. Wang, "A Compact Broadband Mixed-Signal Power Amplifier in Bulk CMOS With Hybrid Class-G and Dynamic Load Trajectory Manipulation," in IEEE Journal of Solid-State Circuits, vol. 52, no. 6, pp. 14631478, June 2017.

[32] E. Kaymaksut and P. Reynaert, "Dual-Mode CMOS Doherty LTE Power Amplifier With Symmetric Hybrid Transformer," in IEEE Journal of Solid-State Circuits, vol. 50, no. 9, pp. 1974-1987, Sept. 2015.

[33] L. Ye, J. Chen, L. Kong, P. Cathelin, E. Alon and A. Niknejad, "A digitally modulated $2.4 \mathrm{GHz}$ WLAN transmitter with integrated phase path and dynamic load modulation in 65nm CMOS," in IEEE International Solid-State Circuits Conference Digest of Technical Papers, 2013, pp. 330-331.

[34] S. Moloudi and A. Abidi, "The Outphasing RF Power Amplifier: A Comprehensive Analysis and a Class-B CMOS Realization," in IEEE Journal of Solid-State Circuits, vol. 48, no. 6, pp. 1357-1369, June 2013.

[35] J. H. Qureshi et al., "A 90-W Peak Power GaN Outphasing Amplifier With Optimum Input Signal Conditioning," in IEEE Transactions on Microwave Theory and Techniques, vol. 57, no. 8, pp. 1925-1935, Aug. 2009.

[36] M. C. A. van Schie, M. P. van der Heijden, M. Acar, A. J. M. de Graauw and L. C. N. de Vreede, "Analysis and design of a wideband high efficiency CMOS outphasing amplifier," 2010 IEEE Radio Frequency Integrated Circuits Symposium, Anaheim, CA, 2010, pp. 399-402.

[37] M. Acar et. al, "Scalable CMOS Power Devices with 70\% PAE and 1, 2 and 3.4 W Output Power at 2GHz", RFIC Symp., pp. 233-236, June 2009.

[38] R. M. Fano, "Theoretical limitations on the broadband matching of arbitrary impedances," J. Franklin Inst., vol. 249, pp. 57-83, 139-155, Jan./Feb. 1950.

[39] Jongchan Kang, A. Hajimiri and Bumman Kim, "A single-chip linear CMOS power amplifier for $2.4 \mathrm{GHz}$ WLAN," 2006 IEEE International Solid State Circuits Conference - Digest of Technical Papers, San Francisco, CA, 2006, pp. 761-769. 


\section{Chapter 6}

\section{Linearity of Outphasing Class-E PAs}

\subsection{Introduction}

Outphasing RF class-E Power Amplifiers (OEPAs) ${ }^{1}$ are efficient switched mode PAs that can benefit from zero voltage switching (ZVS) conditions of the branch class-E PAs to provide high efficiency, both at maximum output power and at back-off [2-6].

Outphasing transmitters in a simplified form, shown in Fig. 6.1(a), consist of a signal component separator (SCS) to convert amplitude modulated signal (shown with phasor $V_{i n}$ ) into two constant envelope phase-only-modulated signals with a phase difference $\Delta \theta_{i n}$, two branch PAs to amplify the phase modulated signals and a combiner to reconstruct the amplified replica of the input signal [6]. Considering phasor representations $V_{\text {out } 1,2}=\left|V_{\text {out } 1,2}\right| e^{j \angle V_{\text {out } 1,2}}$ for the two constant envelope phase modulated signals (where the amplitude and phase information is in $\angle V_{\text {out } 1}-\angle V_{\text {out } 2}$ and $\frac{\angle V_{\text {out } 1}+\angle V_{\text {out } 2}}{2}$, respectively), the output phasor $V_{\text {out }}$ for $\left|V_{\text {out } 1}\right|=\left|V_{\text {out } 2}\right|=V$ can be written as

$$
V_{\text {out }}=\left|V_{\text {out }}\right| e^{j \angle V_{\text {out }}}=2 V \cos \left(\frac{\Delta \theta_{\text {out }}}{2}\right) e^{j \frac{\angle V_{\text {out } 1}+\angle V_{\text {out } 2}}{2}}
$$

where $\Delta \theta_{\text {out }}=\angle V_{\text {out } 1}-\angle V_{\text {out } 2}$ is denoted as the outphasing angle. Assuming that $\Delta \theta_{\text {in }}=\Delta \theta_{\text {out }}$ then for an inverse cosine SCS with $\Delta \theta_{\text {in }}=2 \cos ^{-1}\left(\left|\frac{V_{\text {in }}}{\max \left(V_{\text {in }}\right)}\right|\right)$, the outphasing transmitter shows theoretically linear operation. For this linear operation and to guarantee $\Delta \theta_{\text {in }}=\Delta \theta_{\text {out }}$, (conventionally) an isolating power combiner is used [6]. However, this comes at a cost of low efficiency at power back-off due to

\footnotetext{
${ }^{1}$ This chapter consists of material previously published in IEEE Transactions on Circuits and Systems II (TCAS II) [1] which was re-structured for readability in thesis form.
} 
power dissipation at the isolating port of the combiner. For high efficiency at back-off as well as at maximum output power, non-isolating power combiners are required. Non-isolating combiners ensure high efficiency (ideally 100\%) by providing optimum impedances both at maximum output power power as well as at a specific power back-off level $[4,5]$. Employing non-isolating power combiners, however, results in load-pulling between the two branch PAs that cause input-output phase difference to deviate from $\Delta \theta_{\text {in }}=\Delta \theta_{\text {out }}$ yielding non-linearity (distortion).

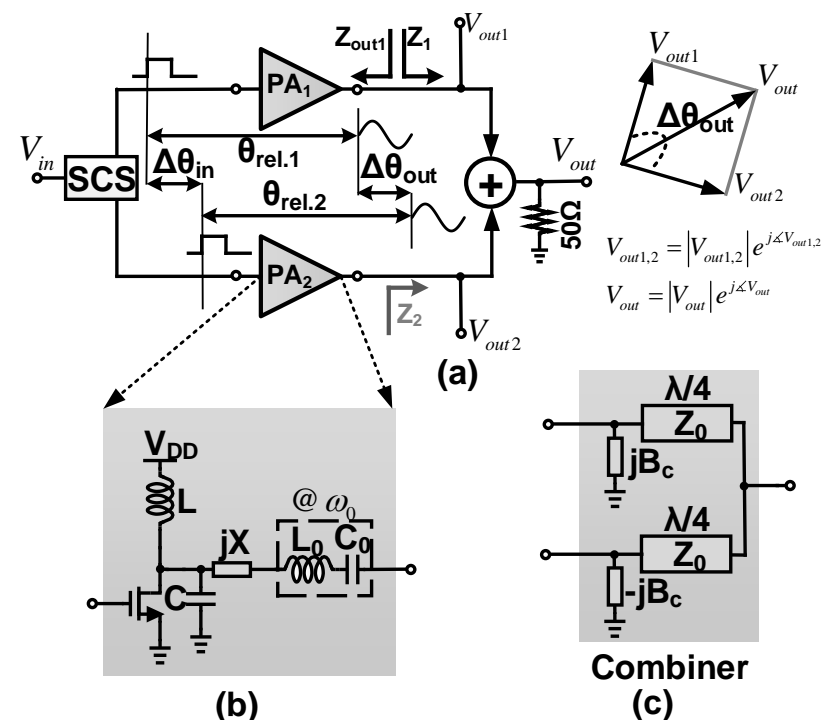

Figure 6.1: (a) Simplified outphasing PA and the phasor representations. (b) Single ended (PA1 and PA2) class-E PA. (c) non-isolating quarter-wavelength transmission-line based combiner.

There are numerous published works on the linearity of outphasing transmitters, e.g. see [7]- [14]. The models developed in [7]- [11] assumed isolating combiners, hence (ideally) linear operation and focus on second order effects causing distortion, e.g. gain/phase mismatch or delay mismatch. Having non-isolating combiners, the non-linearity caused by the load-pulling between the two PAs was recognized in [12] and to the best of authors knowledge was only (theoretically) addressed by [13]. However, the theory in [13] is for the case of linear (e.g. class A) branch PAs; the branch PAs in [13] are modeled as identical voltage sources having a constant output impedance for the full range of outphasing angles. The experimental verifications of the presented model in [13] were reported in [14].

In [5] the authors showed that, depending on the class-E PA parameters and on the OEPA branch amplifiers' load trajectories, identical voltage source modeling of switch mode class-E PAs is valid only under specific conditions. Moreover, in this current 
chapter, we show that the output impedance of a class-E PA is load-dependent which then necessitates a new theoretical model to describe the linearity performance of outphasing transmitters employing class-E PAs as branch amplifiers.

In chapter 5, we presented a general theory of the OEPAs based on the (time domain) load-pull analyses of the branch class-E PAs. Chapter 5 addressed the output power, efficiency, output power dynamic range (OPDR) and reliability related maximum switch voltage while the linearity was not the prime focus of that chapter. In this chapter the linearity of OEPAs is studied theoretically and validated by measurement results of an OEPA employing two class-E branch PAs implemented in a standard $65 \mathrm{~nm}$ CMOS technology and using an off-chip transmission-line based power combiner at $1.8 \mathrm{GHz}$ (similar to chapter 5 ). The theoretical model subsequently is used to define Digital Pre-Distortion (DPD) parameters. The presented theory assumes a so-called load-insensitive OEPA design [4]. However, the presented model can also be applied to any other implementations of OEPAs e.g. to OEPAs with a so-called parallel class-E design [15].

The chapter is organized as follows. Section 6.2 derives the input-output voltage relation (AM/AM and AM/PM distortion) of OEPAs. The measurement results that confirm the presented model are given in section 6.3. This section also presents the measurement results of an OEPA using DPD setting obtained from theory. Finally the conclusions are given in section 6.4 .

\subsection{Theoretical model for the linearity of OEPAs}

The schematic of the branch class-E PAs is shown in Fig. 6.1(b). For each branch class-E PA the MOS transistor acts as a switch that is driven by a square wave input signal with (input) angular frequency $\omega_{0}$ and duty cycle scaling factor $d(d=1$ corresponds to a $50 \%$ dusty cycle). We assume the so-called load-insensitive design for the class-E PAs for which $q=1 / \omega_{0} \sqrt{L C}=1.3$ and $d=1$. For the well-known ZVS and zero slope switching (ZSS) conditions let's assume that $Z_{1}=Z_{2}=R$. Then, the relation between the circuit components $(L, C, X$ and $R), V_{D D}, \omega_{0}$ and output power $P_{\text {out }}$ are given by the so-called K-Design set as [16]

$$
K=\left\{K_{L}, K_{C}, K_{X}, K_{P}\right\}=\left\{\frac{L \omega_{0}}{R}, R C \omega_{0}, \frac{X}{R}, \frac{R P_{\text {out }}}{V_{D D}^{2}}\right\}
$$

For ZVS and ZSS conditions, the K-design set elements only depend on $q, d$ and the switch-on resistance related parameter $m=R_{o n} C \omega_{0}$, e.g. for $q=1.3, d=1$ and $m=0$, we have $\left\{K_{L}, K_{C}, K_{X}, K_{P}\right\}=\{1.04,0.58,0.28,1.26\}$.

The class-E branch PAs (non-linearly) amplify the phase modulated driving signals; after filtering the harmonics by the output series filters $L_{0}-C_{0}$, their output signals $V_{\text {out } 1}$ and $V_{\text {out } 2}$ are fed into the non-isolating combiner. The combiner, shown 
in Fig. 6.1(c), is here assumed to be a transmission-line based power combiner with a characteristic impedance $Z_{0}$ and $\pm j B_{c}$ are the conventional Chireix compensation elements. For the vector diagram, shown in Fig. 6.1(a),

$$
\begin{gathered}
\left|V_{\text {out }}\right|=\sqrt{\left|V_{\text {out } 1}\right|^{2}+\left|V_{\text {out } 2}\right|^{2}+2\left|V_{\text {out } 1} V_{\text {out } 2}\right| \cos \left(\Delta \theta_{\text {out }}\right)} \\
\angle V_{\text {out }}=\tan ^{-1}\left(\frac{\left|V_{\text {out } 1}\right| \sin \left(\angle V_{\text {out } 1}\right)+\left|V_{\text {out } 2}\right| \sin \left(\angle V_{\text {out } 2}\right)}{\left|V_{\text {out } 1}\right| \cos \left(\angle V_{\text {out } 1}\right)+\left|V_{\text {out } 2}\right| \cos \left(\angle V_{\text {out } 2}\right)}\right)
\end{gathered}
$$

which can be readily simplified to (6.1) for $\left|V_{\text {out } 1}\right|=\left|V_{\text {out } 2}\right|=V$.

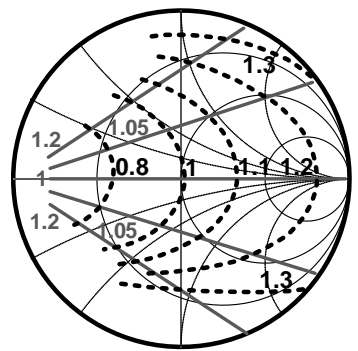

(a)

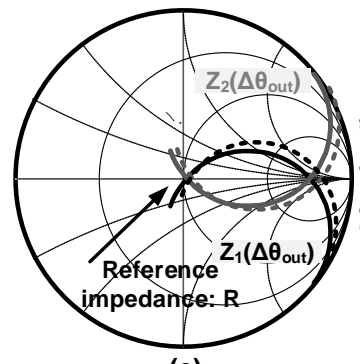

(c)

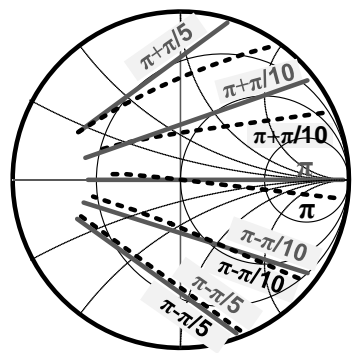

(b)

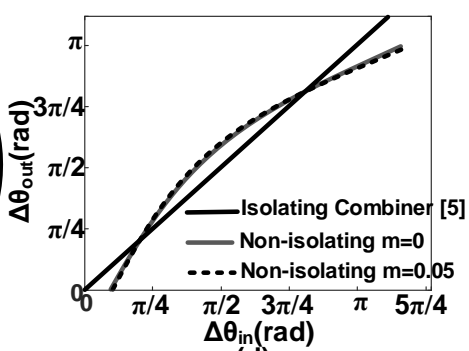

(d)

Figure 6.2: (a) Normalized $\left|V_{\text {out } 1,2}\right|$ contours for $m=0$ (solid-gray) and $m=0.05$ (dotted-dark), (b) $\theta_{\text {rel.1,2 }}$ for $m=0$ (solid-grey) and $m=0.05$ (dotted-dark). (c) PA loads $Z_{1}$ (dark) and $Z_{2}$ (gray) for $m=0$ (solid) and $m=0.05$ (dotted) for $0 \leq \Delta \theta_{\text {out }} \leq \pi$. (d) $\Delta \theta_{\text {out }}$ as a function of $\Delta \theta_{\text {in }}$.

To find $\left|V_{\text {out }}\right|$ as a function of $\left|V_{\text {in }}\right|$ (and therefore to characterize AM/AM distortion), similar to chapter 5 , the load-pull analyses of single branch PAs were leveraged. For this, consider the load-pulling of the top branch class-E PA by changing $Z_{1}$. The mathematical derivation of the output voltage amplitude $\left|V_{\text {out } 1}\right|$ and phase $\angle V_{\text {out } 1}$ is beyond the scope of this chapter and can be found (similar to [16]) by following basic circuit theory. However, these derivations are employed to plot the $\left|V_{\text {out } 1}\right|$ contours normalized to that at nominal load condition $Z_{1}=R$ and the output voltage phase $\angle V_{\text {out } 1}$ with respect to the rising edge of the input driving waveforms (shown with $\theta_{\text {rel.1 }}$ in Fig. 6.1(a)). These contours are shown in Fig. 6.2(a) and (b) for a class-E 
PA with a switch with $m=0$ (ideal loss-less switch) and $m=0.05^{2}$. These contours only depend on the relative load impedance $Z / R$ and are independent of the output power level, $\omega_{0}$ and $R[5]$.

The normalized $\left|V_{\text {out } 1}\right|$ contours in Fig. 6.2(a) show that a class-E PA can not be modelled as a constant voltage source with a constant output impedance. For instance, for $m=0$ (grey-solid contours), on the horizontal axis (for real loads) the output voltage amplitude is not load-dependent and therefore the class-E PA's output impedance, shown in Fig. 6.1 with $Z_{\text {out } 1}$, is zero. But, for non-real loads, the voltage amplitude is load-dependent justifying a non-zero $Z_{\text {out } 1}$. Furthermore, it can be shown that $Z_{\text {out } 1}$ is load dependent and changes across the Smith chart. The normalized $\left|V_{\text {out } 1}\right|$ contours for $m=0.05$ in Fig. 6.2(a) show that for real loads, $Z_{\text {out } 1}$ is also non-zero. As a conclusion, the presented reflection based theoretical model in [13] can not be employed to study OEPAs' linearity and a new model should be developed. The relative output voltage phase contours $\theta_{\text {rel.1 }}$, shown in Fig. 6.2(b) will be used to study the linearity of OEPAs. Having the same $q, d$ and $m$ for both branch class-E PAs, similar contours and discussions hold for lower branch PA.

In [5] it is derived that the apparent PA loads $Z_{1,2}$ can be written as

$$
\frac{1}{Z_{1,2}}= \pm j B_{c}+\frac{R_{L}}{Z_{0}^{2}}\left(1+\left|\frac{V_{\text {out } 2,1}}{V_{\text {out } 1,2}}\right| e^{\mp j \Delta \theta_{\text {out }}}\right)
$$

where we assume $Z_{0}=\sqrt{2 R R_{L}}$ and $B_{c}=1 / 2 R \sin (\pi / 5)$ which corresponds to nulling of the imaginary parts of the PA loads at $10 \mathrm{~dB}$ power back-off [5]. To calculate the apparent PA loads $Z_{1,2}$ for any $\Delta \theta_{\text {out }}$, given in (6.5), the ratio of the (normalized) output voltage amplitudes $\left(\left|\frac{V_{\text {out } 2,1}}{V_{\text {out } 1,2}}\right|\right)$ must be determined. For this, we use an iterative approach similar to that in [5]. That is, we start with $\left|V_{\text {out } 1}\right|=\left|V_{\text {out } 2}\right|$ and calculate the PA loads from (6.5). Based on the calculated PA loads we use the data in Fig. 6.2(a) to update the $\left|\frac{V_{\text {out } 2,1}}{V_{\text {out } 1,2}}\right|$ and to then recalculate the PA loads from (6.5). We stop this iterative routine after reaching a sufficiently low change in $\left|\frac{V_{\text {out } 2,1}}{V_{\text {out } 1,2}}\right|$ (typically less than $1 \%$ error was reached after two or three iterations). The loads of the two branch amplifiers $Z_{1,2}$ are shown in Fig. 6.2(c) for $0 \leq \Delta \theta_{\text {out }} \leq \pi$ and two different values of $m$.

For $m=0$, the PA loads $Z_{1,2}$ are symmetrical with respect to the real axis due to the symmetrical behavior of the normalized $\left|V_{\text {out } 1,2}\right|$ contours. However, the small asymmetrical behavior of the voltage contours with respect to the real axis for $m=0.05$ slightly affects the PA loads and also makes $Z_{2}$ negative for $\Delta \theta_{\text {out }} \rightarrow \pi$ implying that PA2 absorbs a part of the power that PA1 provides. However, due to

\footnotetext{
${ }^{2}$ In [17], it is shown that $m$ only depends on the technology and the operation frequency. For the cascode implemented switch in $65 \mathrm{~nm}$ CMOS technology (in section 6.3), $m=0.05$ shows a fair agreement between theory and simulation results.
} 
the switching and components losses and due to the fact that $P_{\text {out }}>0$ (output power delivered to the $50 \Omega$ load), there is no stability issue here. A complete discussion on the stability can be found in [5].

Overlaying the PA loads $Z_{1,2}$ on the $\theta_{\text {rel.1,2 }}$ contours, the output phase $\angle V_{\text {out } 1,2}$ with respect to the rising edge of the driving input square waveform can be readily obtained. Then the required input phase difference $\Delta \theta_{i n}$ to obtain the phase difference between the output voltages of both branch PAs in an OEPA, $\Delta \theta_{\text {out }}$, is

$$
\Delta \theta_{\text {in }}\left(\Delta \theta_{\text {out }}\right)=\Delta \theta_{\text {out }}-\theta_{\text {rel. } 1}\left(\Delta \theta_{\text {out }}\right)+\theta_{\text {rel. } 2}\left(\Delta \theta_{\text {out }}\right)
$$

Due to the complexity of the load pull equations of class-E PAs, closed form equations for $\theta_{\text {rel. } 1}\left(\Delta \theta_{\text {out }}\right)$ and $\theta_{\text {rel.2 }}\left(\Delta \theta_{\text {out }}\right)$ are not derived in this chapter (if they can be derived at all). Instead, for the (so-called load-insensitive) design parameters $q=1.3$ and $d=1$, we use graphical representations based on the theoretical model in chapter 5. Additionally, a discussion of the impact of the switch on-resistance parameter $m$ is provided. The $\Delta \theta_{\text {out }}$ as a function of $\Delta \theta_{\text {in }}$, shown in Fig. 6.2(d) for $m=0$ and $m=0.05$, shows non-linear behavior which then requires a DPD to achieve linear amplification of amplitude modulated signals. Furthermore, the input-output phase difference relation is hardly affected by the switch conduction loss parameter $m$. For comparison, the corresponding results for isolating combiners are also shown in Fig. $6.2(\mathrm{~d})$ with a solid-dark line [6].

Overlaying the PA loads $Z_{1,2}$ on the $\left|V_{\text {out } 1,2}\right|$ contours, $\left|V_{\text {out } 1}\right|\left(\Delta \theta_{\text {out }}\right)$ and $\left|V_{\text {out } 2}\right|\left(\Delta \theta_{\text {out }}\right)$ are obtained. Then using (6.3) and (6.6) along with a conventional inverse cosine SCS, $\left|V_{\text {out }}\right|$ can be obtained as a function of $\left|V_{\text {in }}\right|$, shown in Fig. 6.3(a). This deterministic $\left|V_{\text {out }}\right|\left(\left|V_{\text {in }}\right|\right)$ is often denoted as AM/AM distortion. Knowing this inherent $\mathrm{AM} / \mathrm{AM}$ relation, the DPD can then be implemented using the inverse of the function $\left|V_{\text {out }}\right|\left(\left|V_{\text {in }}\right|\right)$.

For simplicity, let's assume a differential input phase difference excitation. From Fig. 6.1(a),

$$
\angle V_{\text {out } 1,2}= \pm \frac{\Delta \theta_{\text {in }}}{2}+\theta_{\text {rel. } 1,2}
$$

To find AM to PM distortion, $\left|V_{\text {out } 1,2}\right|\left(\Delta \theta_{\text {out }}\right)$ and $\angle V_{\text {out } 1,2}\left(\Delta \theta_{\text {out }}\right)$ can be applied in (6.4). Then using (6.4), the output phase $\angle V_{\text {out }}$ can be plotted as a function of the input phase difference as shown in Fig. 6.3(b). For $m=0$ (solid line), the contours $\left|V_{\text {out } 1,2}\right|$ and $\theta_{\text {rel.1,2 }}$ are symmetrical with respect to the real axis and therefore the output phase is zero and there is no AM/PM distortion. However, for $m=0.05$, the asymmetrical behavior of the contours results in a non-zero output phase. Due to amplitude imbalance, the output phase approaches $\pi / 2$ for $\Delta \theta_{\text {out }} \rightarrow \pi$.

In addition to the switch conduction loss parameter $m$, there are other second order effects that play a role. In [5], it is shown that the switch voltage is heavily 


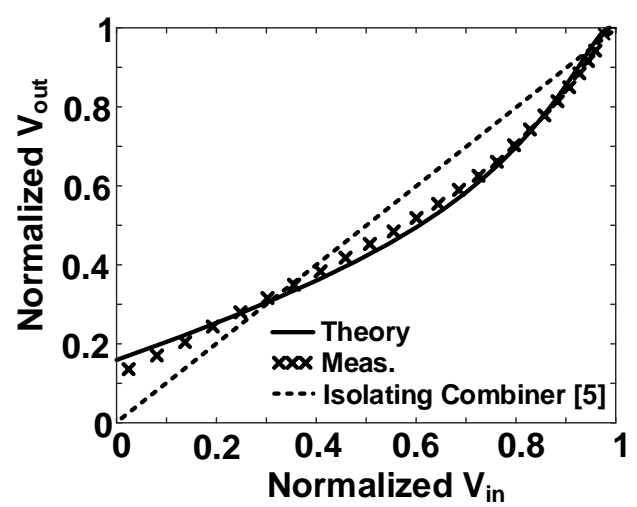

(a)

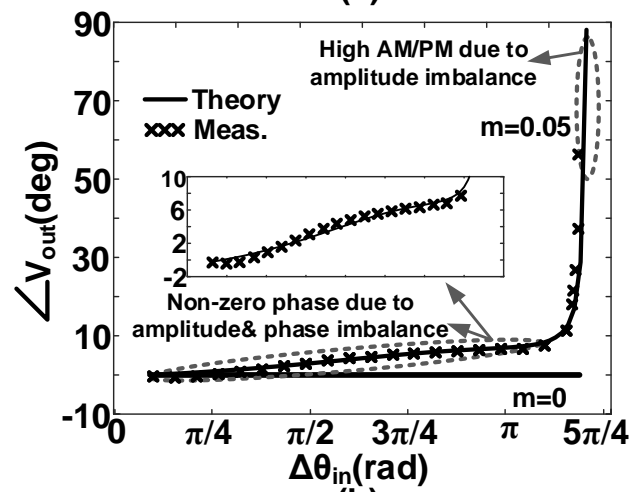

(b)

Figure 6.3: (a) Measured and theoretical $\left|V_{\text {out }}\right|\left(\left|V_{\text {in }}\right|\right)$ for a conventional SCS. (b) Measured and theoretical output phase error versus $\Delta \theta_{i n}$ for $m=0$ (solid) and $m=0.05$ (dotted).

outphasing angle dependent. Since the parameter $q$ of the class-E branch PAs depends on the capacitor $C$ and this capacitor is implemented by parasitic capacitances of the switch, the parameter $q$ is voltage dependent, hence, $\Delta \theta_{\text {out }}$ dependent. In chapter 5 , the impact of changing $q$ on the contours was discussed. Furthermore, imbalance between the two vectors is also due to the limited accuracy of the components in the implementation. Since mismatch is a random phenomenon, we do not study this in details in this chapter; further discussions can be found in e.g. [7]- [11]. To ensure a high output power dynamic range and sufficienctly small AM/PM distortion we reduce the imbalance between the two vectors by e.g. fine-tuning the $q$ parameter of the branch PAs in our measurements [5]. 


\subsection{Experimental verification}

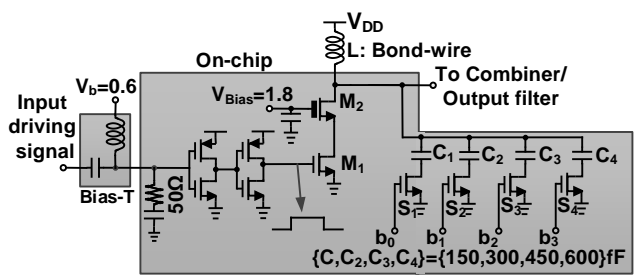

(a)

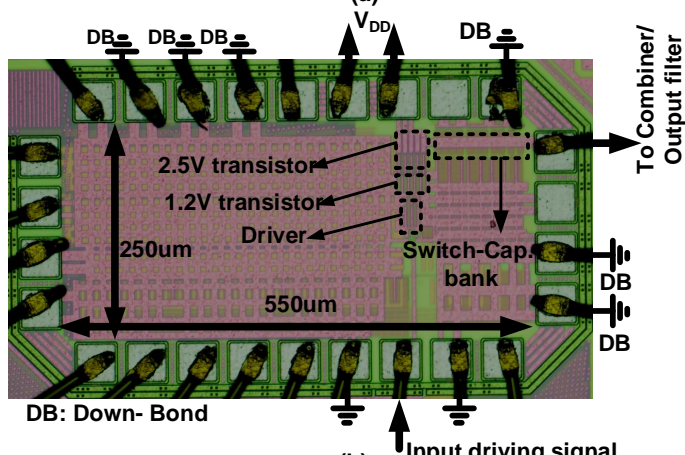

(b)

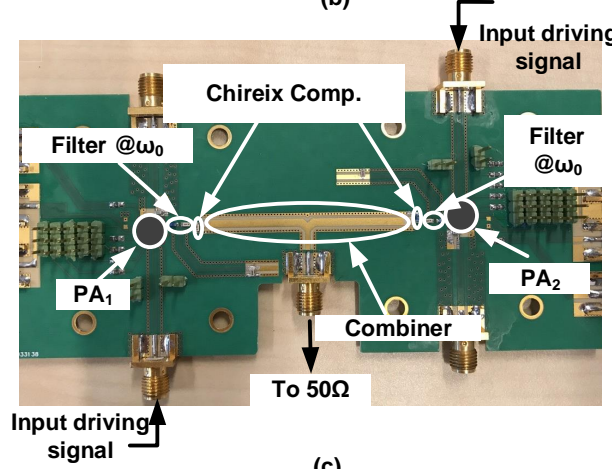

(c)

Figure 6.4: (a) The schematic of the branch class-E PAs. (b) Chip microphotograph. (c) Designed PCB and the combiner.

To validate the presented theory, an OEPA with two branch class-E PAs implemented in a standard $65 \mathrm{~nm}$ CMOS technology and an off-chip quarter-wavelength transmission-line based combiner were used [5], shown in Fig. 6.4. The switch was implemented by a cascode structure where the bottom transistor is a $1.2 \mathrm{~V}$ normal device with aspect ratio $0.84 \mathrm{~mm} / 60 \mathrm{~nm}$ and the cascode tranistor is a thick oxide $2.5 \mathrm{~V}$ device with aspect ratio $1.65 \mathrm{~mm} / 280 \mathrm{~nm}$. Using the K-design set elements for $q=1.3$, $d=1$ and $m=0.05$ and for $R=15 \Omega, \omega_{0}=2 \pi 1.8 \mathrm{GHz}$, yields $L=1.4 \mathrm{nH}, C=3.3 \mathrm{pF}$ and $X=0.5 \mathrm{nH}$. 
A switch/capacitor network was used at the drain node of the cascode to adjust the $q$ parameters of each of the two PAs. The switches in the switch capacitor network are implemented using $2.5 \mathrm{~V}$ thick-oxide transistors and are sized to make sure the maximum voltage across the switches (when they are in off-state) does not exceed (almost) $3 \mathrm{~V}$ for reliability reasons [18]. The chip micro-photograph and the implemented PCB are shown in Fig. 6.4(b) and (c), respectively.

The OEPA provides measured $20.1 \mathrm{dBm}$ maximum output power from a $1.25 \mathrm{~V}$ supply at $1.8 \mathrm{GHz}$ with measured peak drain efficiency (DE) of $65.3 \%$ and peak power added efficiency (PAE) of $60.7 \%$. The results obtained from measurements for $V_{\text {out }}\left(V_{\text {in }}\right)$ (amplitude and phase) assuming a conventional SCS [6] are shown in Fig. 6.3 using crosses. Due to second order effects discussed in section 6.2, small deviations can be observed between the theoretical findings and the measured counterparts. Nevertheless, the measurements are in good agreement with the presented theoretical plots obtained from the previous section. The corresponding results for $\left|V_{\text {out }}\right|\left(\left|V_{\text {in }}\right|\right)$ and for isolating combiners [6] are represented by a dotted line in Fig. 6.3(a). Note that the output phase error for an isolating combiner and perfectly matched branch PAs is zero.

The developed theoretical model of $V_{\text {out }}\left(V_{\text {in }}\right)$ was subsequently used to set the DPD parameters. For this, similar to [5], the polar representation of the IQ time domain signals was used in Matlab. A conventional inverse cosine SCS was used to convert the amplitude information into $\Delta \theta_{i n}$ where the inverse of the function $\left|V_{\text {out }}\right|\left(\left|V_{\text {in }}\right|\right)$ was used before the SCS. After the SCS operation, the theoretical $\angle V_{\text {out }}$ was subtracted from the phase of the both input driving waveforms. The effect of the theory-based DPD on the symbol constellation diagram and on the output power spectral density (PSD) for a single carrier 7-dB PAPR 64QAM signal with 5-MSym/s symbol rate (30-Mbit/s bit-rate) is shown in Fig. 6.5(a) and 6.5(b), respectively. EVM reduction from $-24.6 \mathrm{~dB}$ to $-31 \mathrm{~dB}$ and more than $7 \mathrm{~dB}$ ACLR reduction for $13.1 \mathrm{dBm}$ maximum average output power were measured. The effect of the theorybased DPD on the EVM performance as a function of signal bandwidth (BW) is shown in Fig. 6.6. The measurements show 7dB RMS EVM improvement for $1.25 \mathrm{MHz} \mathrm{BW}$. The EVM at low BW is limited due to second order effects (discussed in section 6.2) that make the measured and theoretical AM/AM and AM/PM distortions to show a small difference. The effectiveness of the implemented memory-less DPD degrades by increasing the BW. Note that this is not a limitation to the theory-based DPD; this is a fundamental limitation of the memory-less DPDs due to their inability to compensate for any memory effects. Discussion on the improving of the EVM at larger BWs can be found in e.g. [5].

Table 6.1 benchmarks the OEPA implementing the presented theory-based DPD against the other previously published works on OEPAs. It can be seen that the implemented OEPA with the theory-based DPD can achieve RMS EVM, ACLR and 


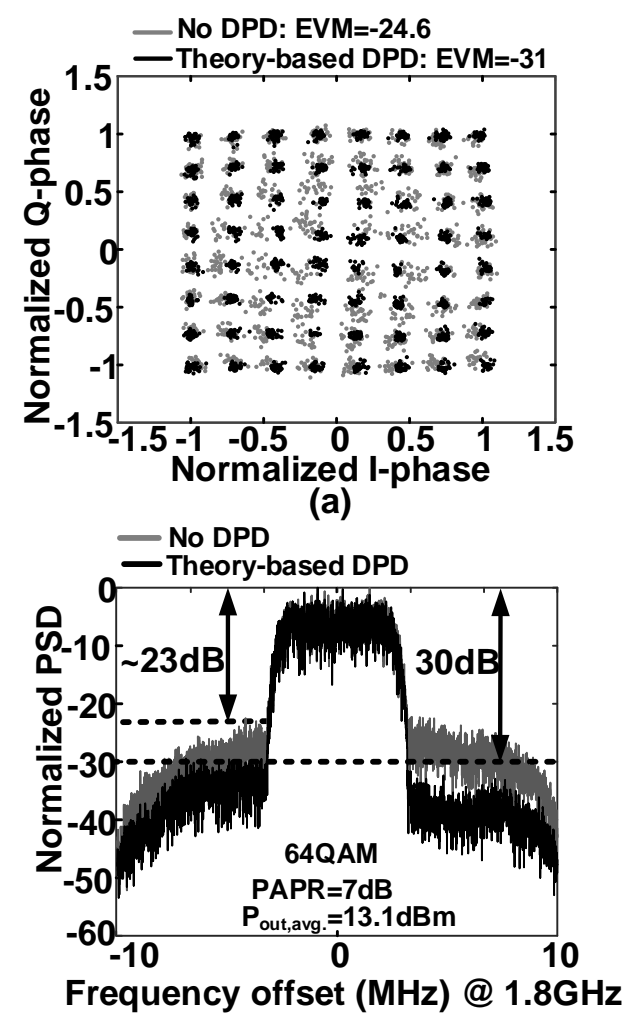

(b)

Figure 6.5: (a) Measured constellation diagram and (b) measured PSD for 5MSym/s (30-Mbit/s) 6.25MHz 64QAM signal at $1.8 \mathrm{GHz}$. The measured DE and PAE are $41.8 \%$ and $33.6 \%$, respectively.

signal fractional BW numbers that are competitive to the works in $[15,19]$. However, leveraging the presented theoretical model to define the DPD parameters omits the conventional need to characterize $\mathrm{AM} / \mathrm{AM}$ and AM/PM distortions.

The theoretical model in this chapter was developed for OEPAs under nominal load conditions. For other load impedances a similar approach can be employed to find the branch PAs' load trajectories hence to find the AM/AM and AM/PM distortions. To compensate for these load-dependent AM/AM and AM/PM distortions, a set of DPD parameters prepared for different antenna load impedances can be used with an adaptive DPD in a feedback loop. If antenna load estimation is possible, for any antenna mismatch, the corresponding DPD setting obtained from the theory can be directly applied.

The theoretical model of this chapter can be further extended by including the 


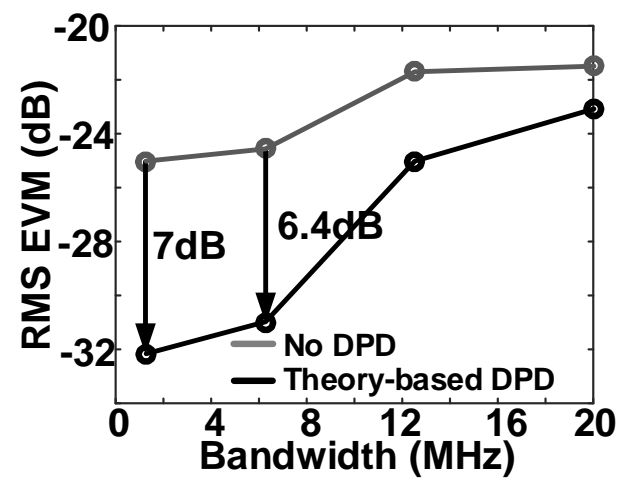

Figure 6.6: Measured RMS EVM as a function of bandwidth for $13.1 \mathrm{dBm}$ 64QAM signal at $1.8 \mathrm{GHz}$.

losses due to the limited quality factor of the dc-feed inductor $\mathrm{L}$ and the output resistance of the switch in off-state. However, this would result in a much more complex model that adds a little accuracy. Such an extension of the model is therefore beyond the scope of this chapter.

Table 6.1: Comparison Table

\begin{tabular}{|c|c|c|c|}
\hline & {$[15]$} & {$[19]$} & The work in this chapter \\
\hline CMOS Tech. & $130(\mathrm{~nm})$ & $40(\mathrm{~nm})$ & $65(\mathrm{~nm})$ \\
\hline Combiner & On-chip & On-chip & Off-chip \\
\hline Freq. (GHz) & 1.85 & 5.9 & 1.8 \\
\hline Supply (V) & 2.8 & 1.2 & 1.25 \\
\hline $\mathrm{P}_{\text {out,Max. }}(\mathrm{dBm})$ & 29.7 & 22.2 & 20.1 \\
\hline Modulation & 16QAM LTE & 64QAM & 64QAM \\
\hline $\operatorname{PAPR}(\mathrm{dB})$ & 7.5 & 7.2 & 7 \\
\hline Fractional BW $(\% 0)$ & 0.54 & 0.34 & 0.35 \\
\hline $\mathrm{P}_{\text {out,avg. }}(\mathrm{dBm})$ & 24.7 & 16.4 & 13.1 \\
\hline $\mathrm{DE}(\%) @ \mathrm{P}_{\text {out,avg. }}$ & Not reported & 23.3 & 41.8 \\
\hline PAE (\%) @ $\mathrm{P}_{\text {out,avg. }}$ & 20.8 & 16.1 & 33.6 \\
\hline RMS EVM (dB) & -30.5 & -30 & -31 \\
\hline $\mathrm{ACLR}(\mathrm{dB})$ & $<-31.6$ & $<-32$ & $<-\mathbf{3 0}$ \\
\hline $\begin{array}{l}\mathrm{AM} / \mathrm{AM} \text { and } \mathrm{AM} / \mathrm{PM} \\
\text { characterization }\end{array}$ & Yes & Yes & No \\
\hline
\end{tabular}




\subsection{Conclusion}

The input-output relation of outphasing class-E PAs (OEPAs) with non-isolating combiners and with a conventional signal component separator exhibits non-linear behavior which was properly modelled in this chapter. This theoretical model was subsequently used to define the DPD parameters for our measurements on an OEPA demonstration. Measurements show that the theory-based DPD enables reaching 31dB EVM and <-30dB ACLR for a single carrier 7-dB PAPR 64QAM signal with 5-MSym/s symbol rate, without requiring the customary AM/AM and AM/PM characterization.

For higher levels of linearity or to compensate e.g. high temperature effects for high power PAs, (normally) a feedback loop to tune the DPD parameters should be employed to fine tune the PA. There, the results of this chapter may provide good initial settings of the DPD in the feedback loop. 


\section{Bibliography}

[1] A. Ghahremani, A. Annema and B. Nauta, "A +20 dBm Highly Efficient Linear Outphasing Class-E PA Without AM/AM and AM/PM Characterization Requirements," in IEEE Transactions on Circuits and Systems II: Express Briefs, vol. 66, no. 7, pp. 1149-1153, July 2019.

[2] Chireix, H., High Power Outphasing Modulation, Proc. IRE, Vol. 23, No. 11, November 1935, pp. 1370-1392.

[3] M. K. Kazimierczuk, "Synthesis of phase-modulated resonant DC/AC inverters and DC/DC convertors," in IEE Proceedings B - Electric Power Applications, vol. 139, no. 4, pp. 387-394, July 1992.

[4] M. P. van der Heijden, M. Acar, J. S. Vromans and D. A. Calvillo-Cortes, "A 19W high-efficiency wide-band CMOS-GaN class-E Chireix RF outphasing power amplifier," 2011 IEEE MTT-S International Microwave Symposium, Baltimore, MD, 2011, pp. 1-4.

[5] A. Ghahremani, A. J. Annema and B. Nauta, "Outphasing Class-E Power Amplifiers: From Theory to Back-Off Efficiency Improvement," in IEEE JSSC, vol. 53, no. 5, pp. 1374-1386, May 2018.

[6] S. C. Cripps, RF Power Amplifiers for Wireless Communications, 2nd ed. Norwood, MA, USA: Artech House, 2006, ch. 10.

[7] X. Zhang, L. E. Larson, and P. M. Asbeck, "Design of Linear RF Outphasing Power Amplifiers". Norwood, MA: Artech House, 2003

[8] A. F. Aref, T. M. Hone and R. Negra, "A Study of the Impact of Delay Mismatch on Linearity of Outphasing Transmitters," in IEEE TCAS I: Regular Papers, vol. 62, no. 1, pp. 254-262, Jan. 2015.

[9] L. Romano, L. Panseri, C. Samori, and A. Lacaita, "Matching requirements in LINC transmitters for OFDM signals," in IEEE TCAS I: Regular Papers, vol. 53, no. 7 , pp. 1572-1578, Jul. 2006. 
[10] J. Guan, A. Aref, T. Hone, and R. Negra, "Linearity study of path imbalances in multi-level LINC transmitter for wideband LTE application," in Proc. Eur. Microw. Conf. (EuMC), Oct. 2013, pp. 728-731.

[11] X. Zhang, L. Larson, P. Asbeck, and P. Nanawa, "Gain/phase imbalanceminimization techniques for LINC transmitters," IEEE TMTT, vol. 49, no. 12, pp. 2507-2516, Dec. 2001

[12] C. P. Conradi, R. H. Johnston and J. G. McRory, "Evaluation of a lossless combiner in a LINC transmitter," in Proc. IEEE Canadian Electrical and Computer Engineering Conf., vol. 1, May 1999, pp. 105-110.

[13] A. Birafane and A. B. Kouki, "On the linearity and efficiency of outphasing microwave amplifiers," in IEEE TMTT, vol. 52, no. 7, pp. 1702-1708, July 2004.

[14] G. Poitau, A. Birafane and A. Kouki, "Experimental characterization of LINC outphasing combiners' efficiency and linearity," Proceedings. 2004 IEEE Radio and Wireless Conference (IEEE Cat. No.04TH8746), 2004, pp. 87-90.

[15] S. Shim and S. Pamarti, "A $1.85 \mathrm{GHz}$ CMOS power amplifier with zerovoltageswitching ontour-based outphasing control to improve back-off efficiency," in Proc. IEEE MTT-S Int. Microw. Symp., Phoenix, AZ, USA, 2015, pp. 1-4.

[16] M. Acar, A. J. Annema and B. Nauta, "Analytical Design Equations for ClassE Power Amplifiers," in IEEE TCAS I: Regular Papers, vol .54, no. 12, pp. 2706-2717, Dec. 2007.

[17] M. Acar, A. J. Annema and B. Nauta, "Analytical Design Equations for Class-E Power Amplifiers with Finite DC-Feed Inductance and Switch On-Resistance," IEEE Circuits Syst. Int. Symp. (ISCAS), New Orleans, LA, 2007, pp. 2818-2821.

[18] A. Mazzanti, L. Larcher, R. Brama and F. Svelto, "Analysis of reliability and power efficiency in cascode class-E PAs," in IEEE Journal of Solid-State Circuits, vol. 41, no. 5, pp. 1222-1229, May 2006.

[19] Z. Hu, L. C. N. de Vreede, M. S. Alavi, D. A. Calvillo-Cortes, R. B. Staszewski and S. He, "A $5.9 \mathrm{GHz}$ RFDAC-based outphasing power amplifier in 40-nm CMOS with $49.2 \%$ efficiency and $22.2 \mathrm{dBm}$ power," in IEEE RFIC, 2016, pp. 206-209. 


\section{Chapter 7}

\section{Augmentation of Class-E PA reliability under load mismatch conditions}

\subsection{Introduction}

Power amplifiers $(\mathrm{PAs})^{1}$ are responsible for a sizable portion of power consumption in RF systems, making PA efficiency an important parameter [2]. For this reason, switched-mode PAs (SMPAs) with their high efficiency are an attractive option. ClassE SMPAs employ resonant networks to shape their waveforms to achieve e.g. zerovoltage switching (ZVS) and/or zero-slope switching (ZSS) conditions to ensure high efficiency (ideally 100\%) [3].

Antenna impedances can vary significantly due to changing antenna environment, with VSWRs as high as 10:1 [4]. Non-nominal load conditions detune the SMPA's resonant networks, in some cases leading to degraded output power and efficiency levels. Additionally, extreme switch voltage and current waveforms can result, jeopardizing PA reliability. Switch voltages exceeding the breakdown level may cause instantaneous and permanent failure through gate-oxide break down [5], while high switch currents can cause gradual deterioration or destruction through electro-migration [6] and hot carrier degradation [7]. The load-pull contours in chapter 5, with focus on outphasing class-E PAs, already demonstrated the effect of load mismatch on the switch voltage of a Class-E PA.

\footnotetext{
${ }^{1}$ This chapter consists of material previously published in 2018 IEEE International Conference on Electronics Circuits and Systems (ICECS) Bordeaux, FRANCE [1] which was re-structured for readability in thesis form.
} 


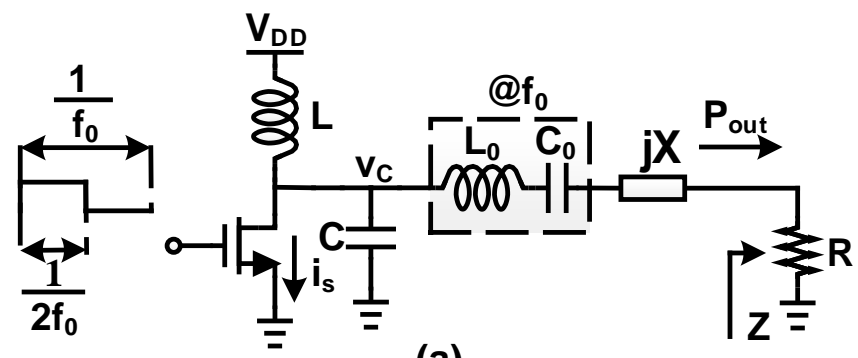

(a)
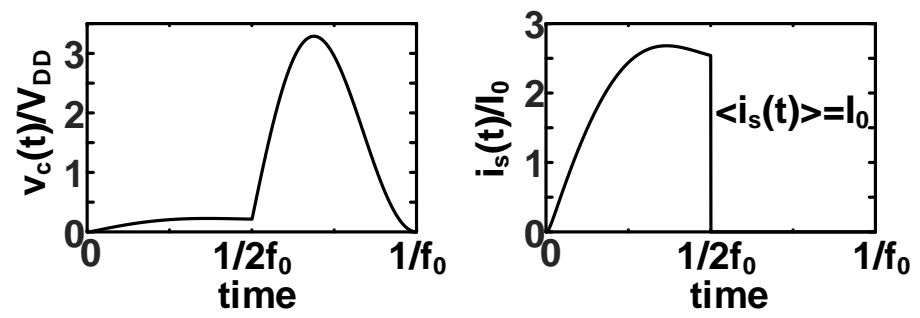

(b)

Figure 7.1: (a) Schematic representation of a single-ended Class-E PA. (b) Switch voltage and current waveforms for nominal load, $\mathrm{m}=0.05, \mathrm{ZVS}$ and ZSS.

There are publications on protecting PAs under non-nominal load conditions. In [9], a tunable matching network is used with a linear Class-A PA to enable selfhealing for high performance under load mismatch. This technique can also be used for protection against load-mismatch, at the cost of reduced efficiency and increased complexity due to need for a tunable network. Another technique, also for linear PAs, involves placing a feedback loop around the PA to detect peak output voltage and use this to tune the bias current of the PA, to ensure both linearity and safe operation of the PA [10]. Voltage/current limiters were also reported for protection [11], [12], at the cost of compromising both PA functionality and efficiency due to e.g. shunting high currents to ground to limit transistor drain voltage. Lastly, isolators/circulators may be used to isolate the PA from mismatches at the load. However, their dissipative operation makes them unsuitable for use in high-efficiency PA systems.

For Class-E PAs, to the best of the authors' knowledge, there is only one work addressing protection against high VSWRs [13]. The work in [13], similar to that on linear PAs, employs a feedback loop to detect high peak voltage at the drain of the switch and reduces the drive power to limit the switch voltage. However, this compromises switch-mode operation (and hence efficiency) because the drive power must be reduced below the level where it adequately switches the switch transistor.

In this chapter, we propose a new technique to improve the reliability of Class-E 
PAs under heavy load mismatch. The technique is based on the rotation of the loadpull contours [8] and hence the safe operating region on the Smith chart, discussed in chapter 5. Furthermore, it is shown that PA efficiency is hardly degraded. Finally, a fully automated self-protective Class-E PA demonstrator is used for experimental verification.

In section 7.2 the general principles and behavior under load mismatch of Class-E PAs are treated. Section 7.2.1 describes our demonstrator implementation and shows measurements on this self-healing system. Section 7.4 summarizes the conclusions.

\subsection{Class-E PAs under (non-)nominal conditions}

Single-ended Class-E PAs, in their simplified form, consist of a switch (transistor) and two LC tanks. A schematic representation of a single-ended Class-E PA can be found in Fig. 7.1. The first tank, $L-C$, is used for waveform shaping and the second tank, $L_{0}-C_{0}$, is a band-pass filter tuned to the input frequency, $f_{0}$, to ensure a sinusoidal load current. In the ideal case, the switch is driven by a $50 \%$ duty cycle square wave with frequency $f_{0}$.

For Class-E PAs designed for ZVS and ZSS conditions, parameters $\alpha$ and $\beta$, which represent the level of violation of ZVS and ZSS conditions, are zero; $\alpha$ and $\beta$ are defined as [7]

$$
v_{c}\left(\frac{1}{f_{0}}\right)=\alpha V_{D D}, \quad \frac{d v_{c}}{d t}\left(\frac{1}{f_{0}}\right)=2 \pi \beta f_{0} V_{D D}
$$

where $v_{c}$ is the switch voltage and $V_{D D}$ is the supply voltage. The relations between the Class-E PA parameters and circuit components $L, C, X$ and $R$ are formulated in e.g. [7], in the so-called K-design set $K=\left\{K_{L}, K_{C}, K_{X}, K_{P}\right\}=\left\{\frac{2 \pi L f_{0}}{R}, 2 \pi f_{0} R C, \frac{X}{R}, \frac{R P_{\text {out }}}{V_{D D}^{2}}\right\}$.

The K-design set parameters depend only, see [7], on $\alpha, \beta$, the relative resonance frequency of the LC tanks, $q=1 / 2 \pi f_{0} \sqrt{L C}$, and the technology dependent relative switch on-resistance, $m=2 \pi f_{0} R_{o n} C$. The only dependencies of $m$ are on the technology and the operation frequency [7].

For a Class-E PA which is designed for a certain $q$ and ZVS and ZSS conditions, load-pull contours can be derived, similar to [8]. The resultant contours show the impact of changing load conditions on PA output power, efficiency and (reliability related) maximum switch voltage and average switch current. To achieve this, the PA load, $Z$ with its nominal value, $R$, corresponding to the center of the Smith charts in Fig. 7.2, is changed and switch voltage and currents can be found using basic circuit theory. While a full derivation is beyond the scope of this chapter, the results are used to plot output power and average switch current normalized to where $Z=R$ $(\mathrm{VSWR}=1: 1)$, efficiency, and maximum switch voltage normalized to $V_{D D}$. These results are illustrated in Fig. 7.2. 
To achieve high output power, $q \approx 1.4$ is taken [7]. For standard $65 \mathrm{~nm}$ CMOS technology in the low $\mathrm{GHz}$ range, $m \approx 0.05$. To represent the behavior of the ClassE PA for a wide range of load conditions, the use of parameter color gradients on a Smith chart is employed. Constant VSWR circles for VSWR $=\{10,5\}$ are also shown.

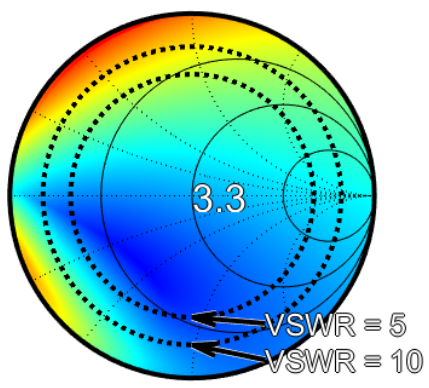

(a)

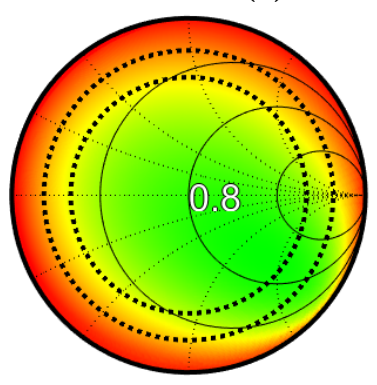

(c)

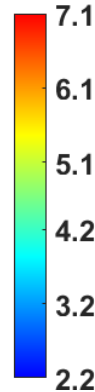

2.2

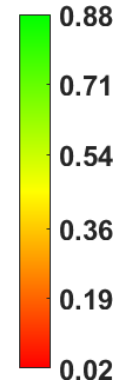

0.02
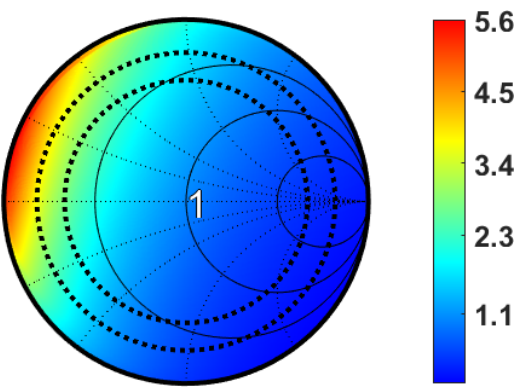

(b)

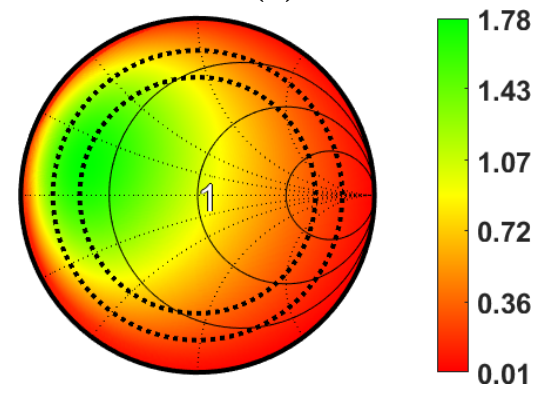

(d)

Figure 7.2: Theoretical Class-E PA load-pull color gradients. (a) Normalized peak switch voltage. (b) Normalized average switch current. (c) Efficiency. (d) Normalized output power.

The contours show that the PA displays peak switch voltages and average switch currents below those for the nominal case for a large portion of the Smith chart, with significantly higher switch voltages and currents mainly occurring in the upper left. Both output power and PA efficiency are heavily affected, reaching near-zero values at their individual extremes. Of the shown effects, breakdown voltage-exceeding switch voltage excursions present the greatest risk to PA reliability, rapidly causing permanent device breakdown and these are therefore the primary focus of the remainder of this chapter. 


\subsubsection{Effects of the $q$ parameter on PA behavior}

In chapter 5 it was shown that reducing (increasing) $q$ rotates the PA contours counterclockwise (clockwise). Since the reliability of the non-tuned PA is compromised mainly in the upper left of the Smith chart, there we reduce $q$ to rotate the contours in the counterclockwise direction. Resultingly, the safe operating area then includes this previously high risk area on the Smith chart. Decreasing $q$ requires that the capacitor $\mathrm{C}$ be controllable to a value larger than the nominal case.

\subsection{Implementation}

To decrease PA sensitivity to load mismatch, the $q$ parameter can be tuned by varying its switch capacitance $C$ when mismatch occurs. A schematic representation of an implementation of the principle, using a switch capacitor bank (SCB), to allow digital control, is shown in Fig. 7.3(a).

To demonstrate the tuning and self-healing principles, measurements were performed on a system reusing the $65 \mathrm{~nm}$ CMOS PA chip described in chapter 5 . The switch consists of a $1.2 \mathrm{~V}$ thin oxide transistor cascoded by a thick oxide $2.5 \mathrm{~V}$ transistor with its gate biased to $1.8 \mathrm{~V}$, allowing a maximum switch voltage of up to $4.6 \mathrm{~V}$ $(1.8 \mathrm{~V}+1.1 \times 2.5 \mathrm{~V}$, with a $10 \%$ technology margin $)$. Using the K-design set elements for $q=1.4, m=0.05$ and $\alpha=\beta=0$ and for $R=25 \Omega, f_{0}=1.4 \mathrm{GHz}$, yields $L=2 \mathrm{nH}, C=3.3 \mathrm{pF}$ and $X \approx 0 j \Omega$. Two parallel bond-wires implement $L$ and the tank capacitor $C$ was implemented as the sum of the drain-bulk and gate-drain parasitic capacitances of the cascode transistor. Two cascaded inverters were used as the switch driver.

The variable switch node capacitor is implemented as a SCB with 4 control bits, with a total $C_{\text {bank }}=1.5 \mathrm{pF}$. The switches are implemented by $2.5 \mathrm{~V}$ thick oxide transistors and dimensioned such that the voltage excursions across them do not exceed $3 \mathrm{~V}$. The extra capacitive loading at the switching node, introduced by the SCB, is compensated by reducing the main switch size, lowering efficiency under nominal load conditions by less than $2 \%$.

A Maury load tuner was used to implement the load-pulling for VSWRs ranging from 1:1 to over 10:1 as shown in Fig 7.3(a). An off-chip matching network was used to step the $50 \Omega$ load down to $R=25 \Omega$. The chip micro-photograph and the designed PCB are shown in Fig 7.3(b) and (c), respectively. For all the measurements in this chapter, the power loss in the matching network is included in our power and efficiency numbers. 


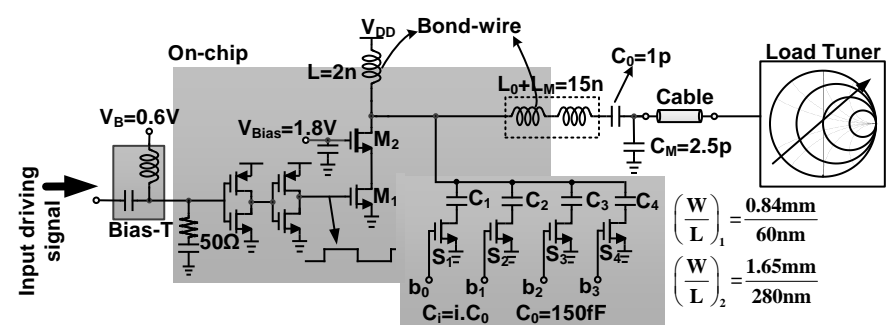

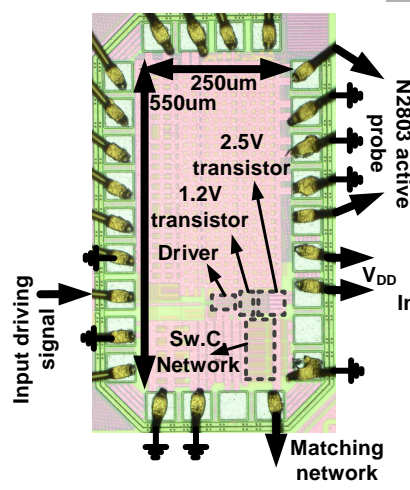

(b)

(a)

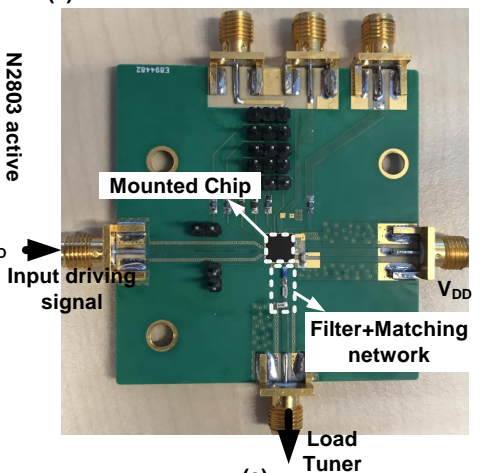

(c)

Figure 7.3: (a) Schematic representation of single-ended Class-E PA with capacitor bank for tuning of the q parameter, matching network and tuner. (b) Chip microphotograph. (c) Designed PCB.

\subsubsection{Measurement results with fixed q parameter}

Under nominal load conditions (VSWR $=1: 1$ ) with no tuning, at $V_{D D}=1.2 \mathrm{~V}$, at 1.4GHz, the PA provides $17.5 \mathrm{dBm}$ into a $50 \Omega$ load at $67 \%$ efficiency.

The PA performance and reliability related parameters were also measured under load-mismatch for a fixed $q=1.4$ (i.e. all the switches in the SCB in the off-state). For reliability and reproducibility reasons, the supply voltage, $V_{D D}$, was varied to keep the PA peak switch voltage below or equal to the peak switch voltage for $Z=R$ to prevent PA breakdown. To equalize the dataset, the data for these loads was scaled to $V_{D D}=1.2 \mathrm{~V}$ during post-processing, assuming $P_{\text {out }} \propto V_{D D}^{2}$, which is valid according to the theory [8]. Additionally, the output power and average switch current data are normalized to the nominal case $(\Gamma=0)$.

Due to the effect of the cables and matching network, the measured contours are rotated with respected to the theory. For clarity sake, the data was counter-rotated during processing to align it to the theoretical contours. The processed measurement results for the PA are shown in Fig. 7.4, where the center of the Smith charts corresponds to $50 \Omega$. Exact mapping between the theory and the measurements is not the 
focus of this chapter. The difference can be explained by second order effects that were not taken into account in our simplified theoretical model. Despite this, the measurement data shows a trend that strongly resembles the theoretical results.

The maximum switch voltage and maximum average switch current under heavy load mismatch can go up to $6.5 \mathrm{~V}\left(5.4 \times V_{D D}\right)$ and $200 \mathrm{~mA}$, respectively, compared to, $V_{c, n o m}=4 \mathrm{~V}\left(3.3 \times V_{D D}\right)$ and $80 \mathrm{~mA}$ under nominal load conditions when $\mathrm{q}$ is fixed. The extreme switch voltage of $6.5 \mathrm{~V}$, toward the upper left of the Smith chart, for a VSWR of 19:1, can instantly break down the PA switch. The border between the safe (peak voltage $\leq 4.6 \mathrm{~V}$ ) and non-safe areas is shown as a solid red line. Additionally, the average current of the switch can reach levels up to 2.5 times higher than under nominal condition, which can gradually degrade switch performance.

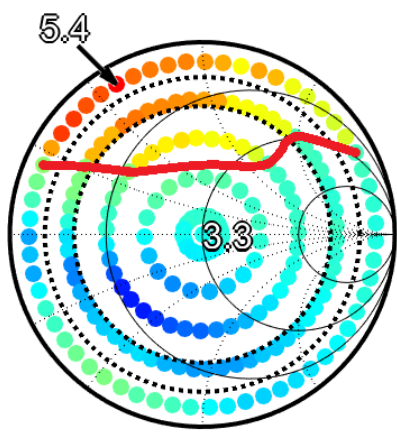

(a)

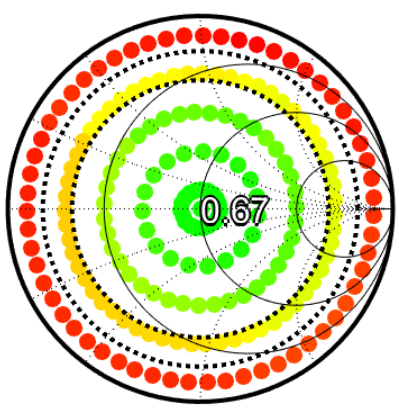

(c)

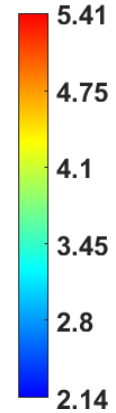

2.14

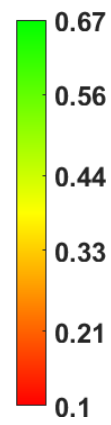

0.1

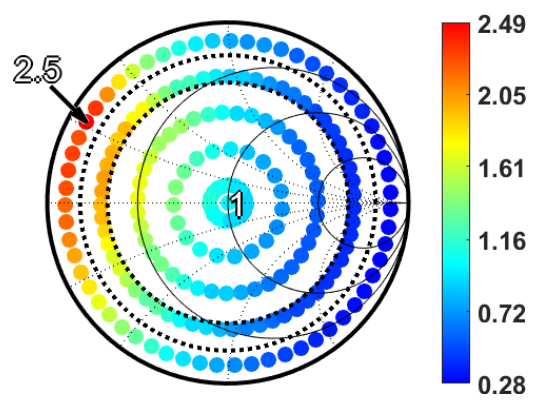

(b)

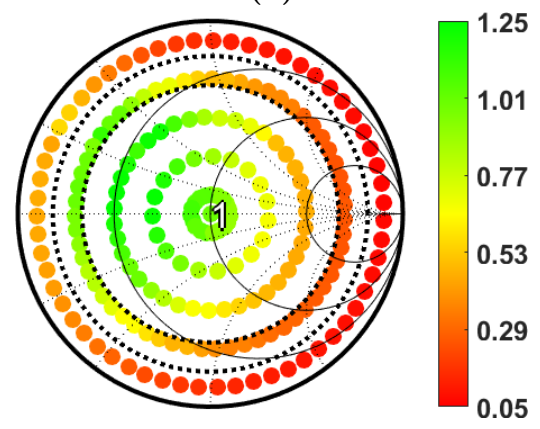

(d)

Figure 7.4: Measured load-pull color gradients with self-heal loop deactivated. (a) Normalized peak switch voltage. (b) Normalized average switch current. (c) Efficiency. (d) Normalized output power. 


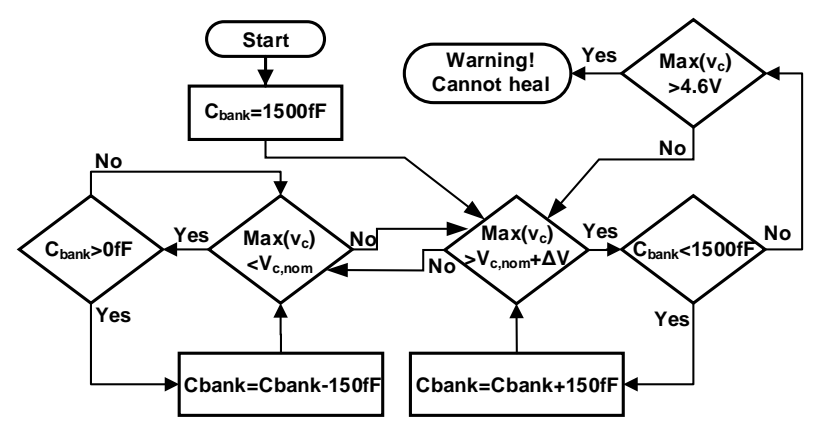

Figure 7.5: Flow diagram of the self-healing algorithm.

\subsubsection{Self-healing Class-E PA}

For self-healing, the $q$ parameter is tuned automatically to extend the safe operating region toward the upper left, with respect to the nominal case $(q=1.4)$. A demonstrator to experimentally validate this is presented below.

For every applied load setting, the waveform at the switch node was measured using an Agilent N2803A active probe connected to an 80GSa/s Agilent oscilloscope. A simple demonstrator algorithm, shown in Fig. 7.5, controls the capacitor bank state. A dead zone of $\Delta V=0.2 \mathrm{~V}$ was added to decrease the noise sensitivity of the self-healing algorithm.

The algorithm checks the maximum switch voltage, $V_{c, \max }$. If $V_{c, \max }<V_{c, \text { nom }}$ , the self-healing loop remains idle. For voltages $V_{c, \max }>V_{c, n o m}+\Delta V=4.2 \mathrm{~V}$, the self-healing loop incrementally increases the SCB state until the peak switch voltage return to a safe value. When the load returns to the nominal condition, the SCB returns to the idle state with all capacitors disconnected. If the SCB is at the maximum state and the peak switch voltage still exceeds the breakdown limit, a warning is given. Fig. 7.6 shows the processed measurement results for the automatic self-healing PA system.

Power and efficiency are hardly affected by the self-healing loop. The worst case deviations in efficiency and output power are an $11 \%$ relative reduction (from $56 \%$ to $50 \%$ ) and a $1.1 \mathrm{~dB}$ reduction (from $79 \mathrm{~mW}$ to $61 \mathrm{~mW}$ ), respectively. The minor efficiency reduction is due to second order effects such as extra loss introduced by the switches in the SCB.

The safe region for instant switch breakdown $\left(\leq 3.8 \times V_{D D}, 4.6 \mathrm{~V}\right)$ is considerably improved extending over the entire measured VSWR range of 19:1. More than 1.4 times reduction in maximum switch voltage can be seen toward the left upper side of the Smith chart. The average switch current safe region is also improved for high 
VSWRs up to 19:1. However, still 2.3 times more average current passes through the switch for extreme load mismatch. Therefore, in the design of the PA a safety factor 2.5 was assumed to ensure the PA can handle the high currents present under heavy load mismatch conditions.

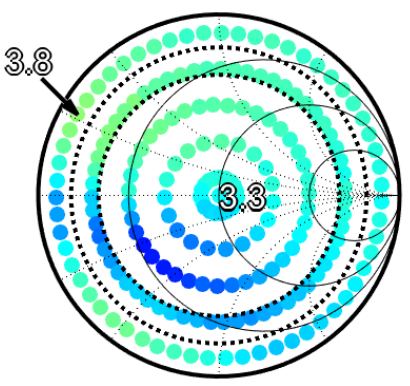

(a)

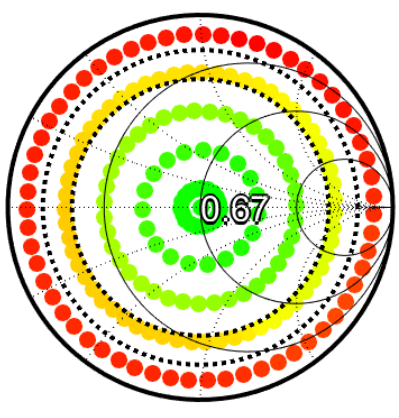

(c)

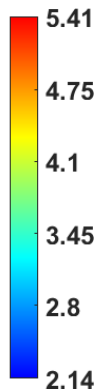

2.14

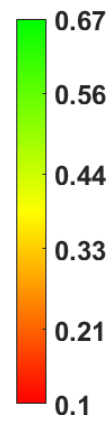

0.1

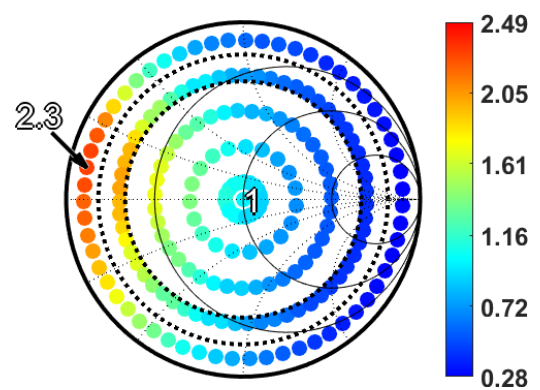

(b)

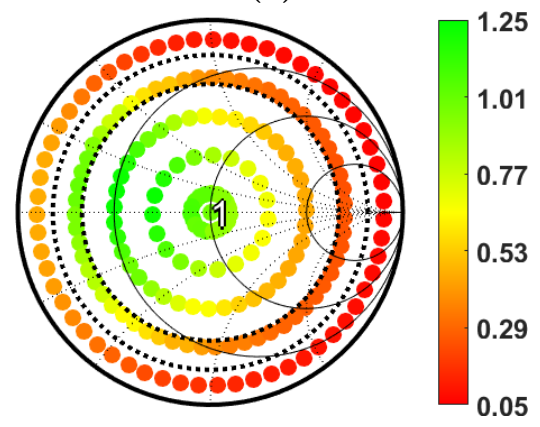

(d)

Figure 7.6: Measured load-pull color gradients with self-heal loop activated. (a) Normalized peak switch voltage. (b) Normalized average switch current. (c) Efficiency. (d) Normalized output power.

\subsection{Conclusions}

The reliability of Class-E PAs can be heavily compromised in the presence of loadmismatch. This chapter introduces, to the authors' best knowledge, the first self protective/healing Class-E PAs. It was shown that decreasing the relative resonance frequency ( $q$ parameter) by employing a digitally variable added switch capacitance, and using a simple algorithm, protected the PA against over-voltage over a wide range of load mismatches with VSWRs as high as 19:1. In so doing, efficiency and output power were not considerably affected. This self-healing Class-E PA in standard 65nm CMOS technology provides good performance and significantly increased immunity to 
load variation related degradation, allowing practical application in systems subject to strong antenna load mismatch with strongly reduced risk to reliability.

A future extension of the work in this chapter will include a receiver-like structure to replace the oscilloscope in the self-protective/healing system. 


\section{Bibliography}

[1] J. Ponte, A. Ghahremani, M. Huiskamp, A. Annema and B. Nauta, "Augmentation of Class-E PA Reliability under Load Mismatch Conditions," 2018 25th IEEE International Conference on Electronics, Circuits and Systems (ICECS), Bordeaux, 2018, pp. 33-36.

[2] S. Cripps, RF Power Amplifiers for Wireless Communications. Artech House, 1999.

[3] F.H. Raab, "Idealized operation of the class-E tuned power amplifier," IEEE TCAS, vol.24, no.12, pp. 725-735, 1977.

[4] K. Boyle and M. Leitner, "Mobile phone antenna impedance variations with realusers and phantoms", Proc. iWAT, pp. 420-423, Hong Kong, 2011.

[5] I. Aoki et al., "A Fully-Integrated Quad-Band GSM/GPRS CMOS Power Amplifier," in IEEE JSSC, vol. 43, no. 12, pp. 2747-2758, 2008.

[6] C. Kim, Electromigration in thin films and electronic devices : Materials and reliability. Oxford: Woodhead Pub.

[7] M. Acar (2011). Power amplifiers in CMOS technology : a contribution to power amplifier theory and techniques, Doctoral dissertatio, University of Twente, Enschede, The Netherlands.

[8] A. Ghahremani et al., "Outphasing Class-E Power Amplifiers: From Theory to Back-Off Efficiency Improvement," in IEEE JSSC, vol. 53, no. 5, pp. 1374-1386, 2018.

[9] S. M. Bowers et al., "Integrated Self-Healing for mm-Wave Power Amplifiers," IEEE T-MTT, vol. 61, no. 3, pp. 1301-1315, 2013.

[10] A. Scuderi et al., "VSWR-protected silicon bipolar power amplifier with smooth power control slope," 2004 IEEE ISSCC, pp. 194-522 Vol.1, San Francisco, CA, 2004. 
[11] W. Karoui and T. Parra, "A protection circuit for HBT RF Power Amplifier under load mismatch conditions," Joint 6th IEEE NWCS and TAISA, Montreal, QC, pp. 241-244, 2008.

[12] N. Zimmermann et al., "An over-voltage protection circuit for CMOS power amplifiers," 15th IEEE ICECS, St. Julien's, pp. 161-164, 2008.

[13] C. Wei et al., "A novel VSWR-protected and controllable CMOS class E power amplifier for Bluetooth applications," 4th IEEE CIEA, Xi'an, pp. 420-424, 2009. 


\section{Chapter 8}

\section{Summary and \\ recommendations for future research}

This chapter summarizes this thesis, and lists the original contributions present in this work. It proceeds with recommendations for future work.

\subsection{Summary and original contributions}

The dominant loss mechanism in linear Power Amplifiers (PAs) such as class A PAs is the power loss of the active device (transistor) due to its overlapping (drain-source) voltage and (drain) current in time domain. The power loss of the passive components (required for matching and biasing) further contributes to the power loss. In SwitchedMode PAs (SMPAs) the power loss of the active unit is mitigated by switch-mode operation of the transistor. In this mode the time in which the non-zero transistor voltage and current overlap is (ideally) eliminated.

The power loss of SMPAs include the loss of the passive components, switching loss that may occur if the voltage across the switch is non-zero at the turn-on moment, switch conduction loss due to non-zero on-resistance of the switch and the power needed to drive the switch. Technology scaling helps to reduce the power loss of the third and fourth mechanisms and with a clever design the switching loss can be reduced to a negligible levels which leaves the passive components loss to be the the physical limitation to achieve high efficiency numbers.

Class-D and class-E SMPAs have attracted a lot of attention for integrated CMOS solutions. Class D PA is a good candidate for low power applications, e.g. $0-10 \mathrm{dBm}$ 
PAs from 0.8 to $1.2 \mathrm{~V}$ nominal supply. Employing class-D PAs in a switched capacitor (SC) structure (so-called SCPA), output power can be easily (yet robust) programmed (for power back-off or amplitude modulations) in a digital manner. Furthermore, the switch voltage excursions in class D PAs are tight to supply and cannot exceed its nominal level, making this class of PAs a robust choice against antenna load variations. However, to achieve a moderate power level of $20 \mathrm{dBm}$ with an acceptable efficiency of the matching network, this class of PAs requires double its nominal supply which may not be always available on-chip. Moderate power level of $10-20 \mathrm{dBm}$ is required in e.g. Bluetooth and WiFi standards.

Class-E PAs, one the other hand, can easily reach to a power level of $20 \mathrm{dBm}$ from a $1.2 \mathrm{~V}$ nominal supply of a standard CMOS technology. This comes with a large voltage excursion (several times of its nominal supply) across the switch transistor which can be handled by employing e.g. cascode structure for the switch. There are yet some concerns to be addressed on class E-PAs.

The voltage excursion across the switch transistor of class-E PAs reaches to about 3.5 times of its nominal supply when designed for the typical $(50 \Omega)$ antenna load. However, antenna load is subject to variation. Load-mismatch sensitivity of class-E PAs was modeled in this thesis mathematically, backed up with circuit simulations and measurements of an implemented class-E PA in 55nm standard CMOS technology. The results of this study showed the variation of the output power, efficiency and reliability of class-E PAs under load-mismatch and helped to identify a safe operating area $(\mathrm{SOA})$ on the Smith chart.

Common techniques to amplitude modulate class-E PAs output signal is to modulate its supply (envelope tracking, envelop elimination and restoration or polar) or to configure two class-E PAs in an outphasing system. Using the developed model for the load-mismatch sensitivity of class-E PAs, the theory of Outphasing class-E PAs (OEPAs) was presented. Furthermore, a technique was presented to improve OEPAs back-off efficiency, reliability under power back-off and output power dynamic range.

Non-isolating power combiners are needed to preserve high efficiency of OEPAs under power back-off. However, the interaction between the two class-E PAs feeding the combiner results in load modulation of the PAs. The load modulation introduces $\mathrm{AM} / \mathrm{AM}$ and $\mathrm{AM} / \mathrm{PM}$ distortions which were mathematically modeled and experimentally verified. The presented model was successfully employed to pre-distort the OEPA without any AM/AM and AM/PM characterization requirements.

Finally, a simple self-protective technique was proposed to maintain class-E PAs reliability under a severe load-mismatch conditions with VSWR up to 19:1 with a minimal degradation on the output power and efficiency. The technique was developed using the presented model for load-mismatch sensitivity of class-E PAs. 


\subsection{Recommendations}

The self-protective class-E PA can be employed in a polar configuration in which the amplitude modulation is provided through a supply modulator. The self-protective loop can affect the phase component of the output signal and needs to be characterized under load-mismatch and corrected in the digital processing unit.

Employing the provided model for the class-E PAs load-mismatch sensitivity, the input-output phase relation as well as the harmonic levels of the output power can be used to estimate the PA loading. This information which can be easily extracted from an integrated waveform characterizer can be used to adjust e.g. a tuneable matching network that corrects for the antenna impedance variations.

The theory of the OEPAs was presented under nominal impedance of the antenna. The developed framework in this thesis facilitates to generalize the theory to include the antenna load variations and propose solutions to improve efficiency, reliability and dynamic range under load variations.

For the design of class-E PAs to be used in a load-insensitive OEPA systems a specific relative resonance tank frequency of $q=1.3$ and $50 \%$ duty cycle were used. These specific design parameters give the desired load-pull contours required for OEPA operation. It was also shown that the contours rotate by changing $q$ and the duty cycle while the shape of the contours was hardly affected. This opens a great design space for OEPAs to explore different combinations for the parameter $q$ and the duty cycle per application. For example at high frequencies where the switch conduction loss increases (due to the limitation of the transistor sizing) choosing a smaller (than 50\%) duty cycle improves the output power at a better efficiency and a lower switch voltage stress.

Finally, for voltage mode OEPAs a transformer is needed. Eliminating the transformer and combining the power in current domain, the load trajectories of the class-E PAs are affected. A new combination of the parameters $q$ and duty cycle can be found to provide the maximum efficiency (ideally 100\%), e.g. $q=1.85$ for $50 \%$ duty cycle. For $q=1.85$ the output power capability of the class-E PAs drops w.r.t. $q=1.3$ which makes it suitable for low power applications. At high frequencies where the switch conduction loss increases, choosing $q=1.85$ over the $q=1.3$, offers a better efficiency. 


\section{Appendix A}

\section{Effect of PA impedance at the harmonics}

The load tuner and the PA impedance settings at the fundamental harmonic were already shown in Fig. 4.14(b). In section 4.4 .3 a deterministic impedance at the harmonics of the operating frequency was assumed for each load mismatch. However, measurements of the tuner impedance (at cross section AA in Fig. 4.14(a)) at the harmonics of the operating frequency reveals that for a mismatch with reflection coefficient amplitude $|\Gamma|$ at the fundamental harmonic, the amplitude of the reflection coefficient at the second and third harmonic can be in the range of $\leq|\Gamma|$. Assuming an arbitrary (and unknown) connection length (between the matching network and the antenna) $\angle \Gamma$ can be any arbitrary angle. Therefore, it would be more practical to assume that the PA load at second (and third) harmonic can occur at any place on the Smith chart. In our work the effects of the higher than third harmonic are negligible.

To examine the effects of the load current at the harmonics on the performance, we assume a load mismatch $\Gamma=0.82$ at the fundamental harmonic which is (close to) a worst case situation for sensitivity to harmonics among any mismatch yielding a VSWR $\leq 10$. Then, we sweep the PA impedance at the second and third harmonics across the Smith chart in the range $|\Gamma| \leq 0.9$ and then compare the simulated performance metrics with that of the calculated counterpart. For calculations (see section 4.4.3), an infinite $Q_{\text {loaded }}$ was assumed (no load current at the harmonics). For simulations the circuit with the components (section 4.4.3) was employed. The loaded quality factor $Q_{\text {loaded }}$ at the fundamental harmonic and for the nominal load was set to 5 .

The results of these simulations (for the second harmonic) are shown in Fig. A.1. Fig. A.1(a), (b) and (c) show the relative error between the simulations and the theory 
for the output power, efficiency and normalized maximum switch voltage, respectively. The maximum relative error in the output power and the maximum switch voltage is $10 \%$ while the efficiency is more sensitive to the PA load at the second harmonic. It can be seen that maximum error occurs at the right lower corner on the Smith chart. In this region, the capacitive part of the load at the second harmonic nulls the effect of the inductive impedance of the series filter and as a result reduces the PA impedance at the second harmonic.

Changing the third harmonic for the same mismatch on the fundamental, similar plots can be obtained. The maximum relative errors on the output power, efficiency and the maximum switch voltage are lower than $1.5 \%$. Similar plots can be generated for the other mismatches on the fundamental harmonic. For instance for the nominal load at the fundamental frequency, sweeping the PA load at the second harmonic in the range $|\Gamma| \leq 0.9$, results in atmost $8 \%$ error in the efficiency.

In conclusion, including harmonics in the load current would result in a much more complex model that adds a little accuracy in (especially) a few regions on the Smith chart. Such an extension of the model is, however, beyond the scope of this work. 


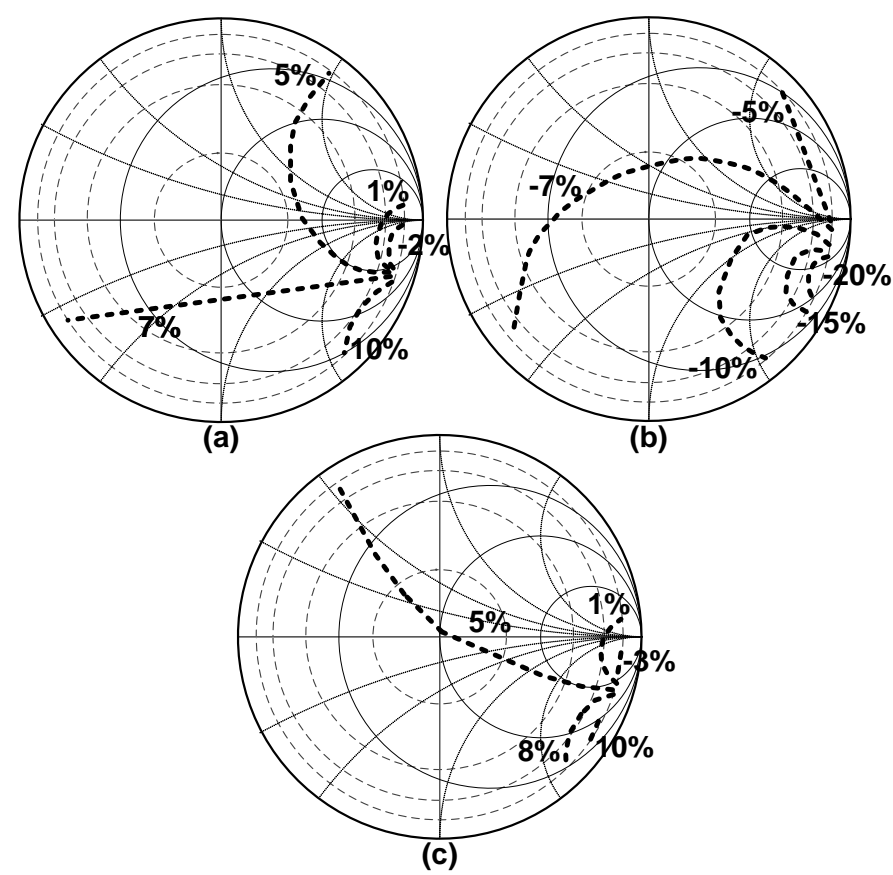

Figure A.1: Relative error between the simulations and the theoretical results due to the PA load change at the second harmonic for the mismatch on the first harmonic with $\Gamma=0.82$. (a) output power, (b) efficiency, (c) maximum switch voltage. 
A. EFfect of PA Impedance AT the haRmonics 


\section{Appendix B}

\section{Effect of changing duty cycle on the load-pull contours}

The effects of changing parameter $\mathrm{d}$ from 1 to 0.7 , respectively to 1.2 on the load-pull contours are shown in Fig. B.1; clockwise (anti-clockwise) rotation occurs for lower (higher) $d$. Note that the normalized maximum switch voltage as well as the normalized output voltage amplitude contours change with the rotation. This can potentially impact the OEPAs reliability and the OPDR which were taken into account in our efficiency improvement technique. 

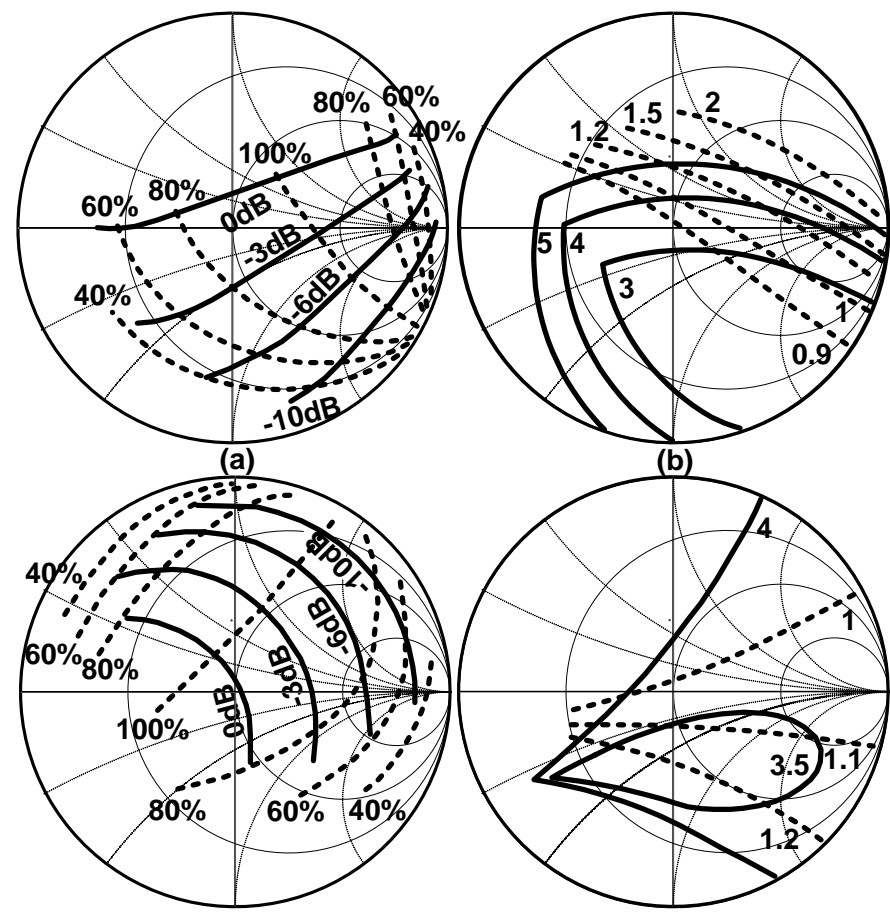

(c)

(d)

Figure B.1: Effect of changing d from 1 to $0.7(\mathrm{a}, \mathrm{b})$, respectively 1.2 (c,d) on the load-pull plots for $q=1.3$ and $m=0$. (a,c) Normalized output power (solid) and efficiency (dotted) contours. (c,d) $V_{c, \text { Max }}$. normalized to $V_{D D}$ (solid) and normalized output voltage amplitude (dotted). 


\section{Appendix C}

\section{Extension of the load-pull contours toward negative impedances}

The load-pull contours of a class-E PA with load-insensitive design, including negative impedances, are shown in Fig. C.1. Outside the Smith chart, the real part of the load is negative and the direction of the output power is toward the switch (negative $\left.P_{\text {out }}\right)$. Positive efficiencies $(\eta>100 \%)$ imply that the supply voltage $V_{D D}$ sinks a fraction of the power that comes from the load (negative $P_{s}$ ). Since the switch loss is always positive, then $\left|P_{s}\right|<\left|P_{\text {out }}\right|$ which results in efficiencies more than $100 \%$. Infinite efficiency shows that the whole power that comes from the load is dissipated in the switch and $P_{s}=0$.

Negative efficiecies show that both the load power and the supply power are dissipated in the switch. Fig. C.1, shows an intersection point where all the efficiency contours converge. At this point the load in open, $P_{\text {out }}=0$, there is no loss in the switch, which then results in $P_{s}=0$. Therefore, at this point the efficiency is not defined; $\eta=\frac{P_{\text {out }}}{P_{s}}=\frac{0}{0}$. 


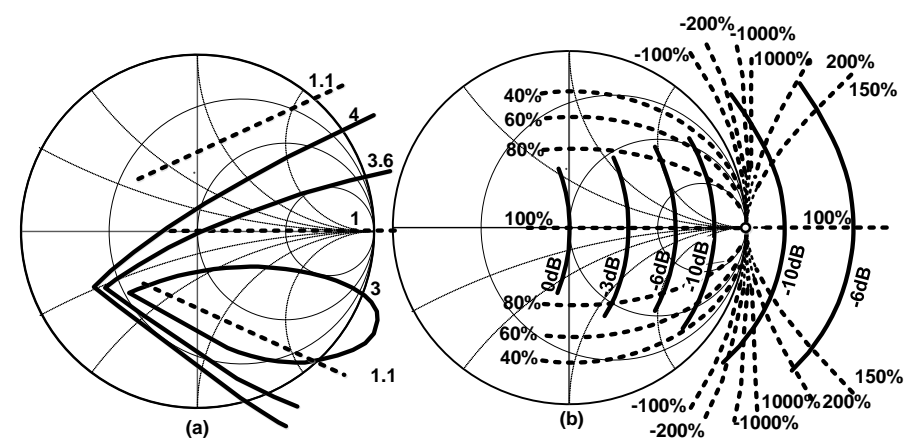

Figure C.1: Load-pull contours of a class-E PA with load-insensitive design and $m=0$. (a) Maximum switch voltage normalized to $V_{D D}$ (solid) and normalized output voltage amplitude (dotted), (b) normalized output power (solid) and efficiency (dotted) contours. 


\section{Acknowledgments}

I would not have accomplished this milestone if I did not have support from so many people during this journey.

First of all, I would like to express my gratitude to my promoter Bram Nauta for giving me the opportunity to do my $\mathrm{PhD}$ in his research group. Bram has created a positive work atmosphere in his team that everybody feels comfortable to ask for help, discuss technical problems and share ideas. His enthusiasm for detailed discussions during and his encouragement after our meetings were very motivating.

My sincere thanks go to my co-promoter Anne-Johan Annema. He was very supportive to me to get started in the Netherlands and of a great help during my PhD. Looking back to my first year at ICD, I see an enthusiastic student with wild ideas who needed a patient and experienced supervisor and I believe I was very lucky to have you supervising me. The most productive times for me were those we had technical discussions that led to the ideas that we could implement and publish some of those. Anne-Johan, working with you was really fun.

My gratitude also goes to the other graduation committee members for taking their valuable time to read and comment the thesis; Frank van Vliet for the discussions we had on the design and tuning of off-chip power combiners, Peter Baltus for offering me a work place at TU Eindhoven while I was working on my thesis, Braham Ferreira and Leo de Vreede for taking time to scrutinize my thesis.

I would like to thank my user committee members, Herma van Kranenburg (NWO), William Aartsen (Dialog), John Willms (Dialog), Rik Jos (Macom), Freek van Straten (Macom), Eisse Mensink (ItoM) and Gerard Voshaar (Bruco). Their expertise and valuable comments undoubtedly helped and enhanced the formulation of this thesis.

I would like to thank Eric Klumperink for the technical discussions we had before the tape-outs. I had the opportunity to assist him in Advanced Analog Integrated Circuit design course and to replace him when he was attending conferences. I found Eric a patient teacher whom I learnt some essential tricks from to keep students motivated during lectures. One of the memorable times with Eric was the group dinner we had in Hawaii in an Indonesian restaurant.

I would like to acknowledge other ICD staff, Ronan van der Zee, Daniel Schinkel, 
Mark Oude Alink and Sander Gierkink for their inputs in my design reviews. I would like to profusely thank my colleagues, Sajad Golabi, Dirk-Jan van den Broek, Johan Withagen, Hugo Westerveld, Ines Inacio, Claudia Palattella, Harijot Singh Bindra, Maikel Huiskamp, Joeri Lechevallier, Yuan-Ching Lien, Vijaya Purushothaman, Qiao Zhiliang, Bart Thijssen, Chris Lokin, Anoop Bhat, Joep Zanen and Jeroen Ponte. I enjoyed having lunch/brain storming sessions with you. A special thank to Sajad Golabi for letting me stay at his place in the last year of my $\mathrm{PhD}$, to Henk de Vries for supporting me during lab measurements and to Gerard de Wienk for support in the last days of tape-outs. And, Gerdien, thank you for all the secretarial support. Seeing your smile in the mornings always cheered me up.

My family also deserve to be mentioned. Many thanks to them, especially to my parents for sacrificing many things in their own life to raise seven children. It was a difficult decision to leave them and I am grateful for their support.

And, last but not least, I would like to express my gratitude to my wife Shokoufeh. On midway of my study we decided to move to Eindhoven. Shokoufeh, traveling 2-3 time a week was frustrating and I truly believe without your help and your everyday support this would not have been possible. My love to you grows stronger everyday and I feel fortunate to have you and our lovely son Nickan. 


\section{List of publications}

- A. Ghahremani, A. Annema and B. Nauta, "A 20dBm outphasing class E PA with high efficiency at power back-off in 65nm CMOS technology," 2017 IEEE Radio Frequency Integrated Circuits Symposium (RFIC), Honolulu, HI, 2017, pp. 340-343.

- A. Ghahremani, A. Annema and B. Nauta, "Load Mismatch Sensitivity of Class-E Power Amplifiers," in IEEE Transactions on Microwave Theory and Techniques, vol. 67, no. 1, pp. 216-230, Jan. 2019.

- A. Ghahremani, A. Annema and B. Nauta, "Outphasing Class-E Power Amplifiers: From Theory to Back-Off Efficiency Improvement," in IEEE Journal of Solid-State Circuits, vol. 53, no. 5, pp. 1374-1386, May 2018.

- A. Ghahremani, A. Annema and B. Nauta, "A +20 dBm Highly Efficient Linear Outphasing Class-E PA Without AM/AM and AM/PM Characterization Requirements," in IEEE Transactions on Circuits and Systems II: Express Briefs, vol. 66, no. 7, pp. 1149-1153, July 2019.

- J. Ponte, A. Ghahremani, M. Huiskamp, A. Annema and B. Nauta, "Augmentation of Class-E PA Reliability under Load Mismatch Conditions," 2018 25th IEEE International Conference on Electronics, Circuits and Systems (ICECS), Bordeaux, 2018, pp. 33-36.

- J. Ponte, A. Ghahremani, M. Huiskamp, A. Annema and B. Nauta, "Theory and Implementation of a Load-Mismatch Protective Class-E PA System," in IEEE Transactions on Circuits and Systems I: Regular Papers.

- A. Khodkumbhe, M. Huiskamp, A. Ghahremani, B. Nauta, A. Annema "Preserving Polar Modulated Class-E Power Amplifier Linearity Under Load Mismatch," 2020 IEEE Radio Frequency Integrated Circuits Symposium (RFIC), Los Angeles, CA, 21-23 June, 2020. 